\title{
Multiuser Multirate DS-CDMA Detection Techniques in Multipath Fading Channels
}

\author{
by \\ Bin Yang
}

A thesis submitted to the Faculty of Graduate Studies and Research in partial fulfillment to the requirements for the degree of

Doctor of Philosophy

Ottawa-Carleton Institute for Electrical and Computer Engineering Department of Systems and Computer Engineering

Carleton University

Ottawa, Ontario, Canada

May 2007

(C)2007 Bin Yang 


$\begin{array}{ll}\begin{array}{l}\text { Library and } \\ \text { Archives Canada }\end{array} & \begin{array}{l}\text { Bibliothèque et } \\ \text { Archives Canada }\end{array} \\ \begin{array}{l}\text { Published Heritage } \\ \text { Branch }\end{array} & \begin{array}{l}\text { Direction du } \\ \text { Patrimoine de l'édition }\end{array} \\ \begin{array}{l}\text { 395 Wellington Street } \\ \text { Ottawa ON K1A ON4 }\end{array} & \begin{array}{l}\text { 395, rue Wellington } \\ \text { Ottawa ON K1A ON4 } \\ \text { Canada }\end{array}\end{array}$

Your file Votre référence ISBN: 978-0-494-27118-6 Our file Notre référence ISBN: 978-0-494-27118-6

NOTICE:

The author has granted a nonexclusive license allowing Library and Archives Canada to reproduce, publish, archive, preserve, conserve, communicate to the public by telecommunication or on the Internet, loan, distribute and sell theses worldwide, for commercial or noncommercial purposes, in microform, paper, electronic and/or any other formats.

The author retains copyright ownership and moral rights in this thesis. Neither the thesis nor substantial extracts from it may be printed or otherwise reproduced without the author's permission.
AVIS:

L'auteur a accordé une licence non exclusive permettant à la Bibliothèque et Archives Canada de reproduire, publier, archiver, sauvegarder, conserver, transmettre au public par télécommunication ou par l'Internet, prêter, distribuer et vendre des thèses partout dans le monde, à des fins commerciales ou autres, sur support microforme, papier, électronique et/ou autres formats.

L'auteur conserve la propriété du droit d'auteur et des droits moraux qui protège cette thèse. $\mathrm{Ni}$ la thèse ni des extraits substantiels de celle-ci ne doivent être imprimés ou autrement reproduits sans son autorisation.
In compliance with the Canadian

Privacy Act some supporting forms may have been removed from this thesis.

While these forms may be included in the document page count, their removal does not represent any loss of content from the thesis.
Conformément à la loi canadienne sur la protection de la vie privée, quelques formulaires secondaires ont été enlevés de cette thèse.

Bien que ces formulaires aient inclus dans la pagination, il n'y aura aucun contenu manquant.

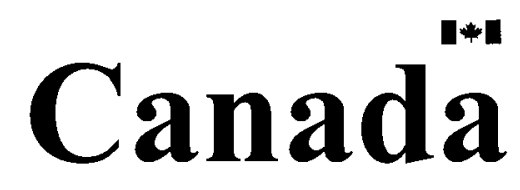




\begin{abstract}
Code division multiple access (CDMA) is a promising technique for wireless communication where multiple users share the same channel, but is subject to intersymbol interference (ISI), multiple access interference (MAI) and near-far effects under multipath channels. This thesis investigates various multirate multiuser detection techniques (MUD) to mitigate these interferences in multirate Direct Sequence (DS) CDMA communication systems over multipath Rayleigh fading channels.
\end{abstract}

A new decorrelator based successive interference cancellation (DBSIC) multiuser Rake receiver is proposed for asynchronous Variable Processing Gain (VPG) multirate DS-CDMA systems. By including a decorrelator on top of matched filtering (MF) at each stage of the conventional SIC to determine the user's signal to be used at the next cancellation stage, DBSIC improves the overall system performance at the expense of feasible additional complexity.

Bit-error-rate (BER) performance of DBSIC is presented using an analytical approach and simulations. DBSIC improvement over other commonly used suboptimum MUD such as the decorrelating, minimum mean-square-error (MMSE), SIC, parallel interference cancellation (PIC) and decorrelating decision-feedback (decorrelating DF) detectors is demonstrated under both perfect and imperfect channel estimation.

It is shown that DBSIC outperforms all other considered MUD in various multirate scenarios even when some physical users have increased data rates (i.e. heavier system's load in terms of the number of virtual users). It is observed that, while all the receivers suffer performance degradation under imperfect channel estimation, DBSIC still outperforms the other schemes.

DBSIC effectiveness to combat MAI is also evaluated in terms of its asymptotic multiuser efficiency (AME). AME for DBSIC is derived and compared with that of 
conventional single-user MF, optimum, decorrelating and SIC receivers over AWGN and Rayleigh channels.

To combat effectively the effects of multipath propagation and MAI, most MUD require some channel state information (CSI). Their performances depend mainly on their ability to obtain an accurate estimation of the CSI they require. An adaptive MAI-resistant joint channel estimation and MUD method combining decision-directed and data-aided approaches is developed for asynchronous multirate CDMA systems. The effectiveness of this joint scheme is supported by results in terms of channel estimates MSE and BER performance. 


\section{Acknowledgments}

First of all, I would like to honor my late father with this thesis. His encouragement, love and support gave me confidence and drive for pursuing my $\mathrm{PhD}$. I would also like to thank my mother for her unconditional love and support throughout the period of my study.

I would like to take this opportunity to thank my supervisor, Prof. Florence Danilo-Lemoine for her insights, guidance, valuable input, attention to detail and editorial effort for the research project and the writing of this thesis throughout my study at Carleton.

I also wish to thank all the members of my comprehensive examining board for giving me constructive suggestions and advices.

Thanks are extended to my brothers and their families, my friends for their support and many others who have made my time at Carleton truly enjoyable.

Finally, and most importantly, I am completely indebted to my wife and daughter for their love, support, patience, cooperation and understanding which make the last few years so memorable.

To my late father. 


\section{Contents}

1 Introduction 1

1.1 Thesis Objectives . . . . . . . . . . . . . . . 1

1.2 Thesis Contributions ................... 5

1.3 Thesis Organization . . . . . . . . . . . . . . 7

2 Literature Review $\quad 9$

2.1 Literature review on multiuser detection . . . . . . . . . . 9

2.1.1 Introduction: single-rate versus multi-rate CDMA systems . . 9

2.1.2 Single-rate multiuser systems . . . . . . . . . . . . . 13

2.1.3 Multi-rate multiuser systems . . . . . . . . . . . . 20

2.1.4 Power control in DS-CDMA systems . . . . . . . . . 26

2.1.5 AME performance .................. 26

2.2 Literature review on channel estimation . . . . . . . . . . . 28

3 Multiuser Multirate Asynchronous DS-CDMA Receivers 37

3.1 Transmitted signals for a multirate VPG CDMA system . . . . . . . 37

3.2 Channel model and received signal . . . . . . . . . . . . 46

3.3 Proposed DBSIC receiver . . . . . . . . . . . . . 53

3.4 Commonly used multiuser detectors . . . . . . . . . . . . . . 62 
3.5 Receivers'computational complexity ............... 64

3.6 Implementation of linear decorrelating detector with reduced complexity 67

3.7 Modified PIC ....................... 71

4 Joint Channel Estimation and Multiuser Detection $\quad 74$

4.1 Introduction . . . . . . . . . . . . . . . . . 74

4.2 Joint channel estimation and multiuser detection . . . . . . . . 76

5 Analytical Performance Analysis $\quad 87$

5.1 BER performance analysis . . . . . . . . . . . . . . 87

5.2 AME performance analysis . . . . . . . . . . . . . . 92

5.2.1 System models and receiver structures for synchronous DS-CDMA systems ................................. 92

5.2.2 AME performance analysis over AWGN channels . . . . . . 95

5.2.3 AME performance analysis over a Rayleigh flat fading channel 99

6 Simulation Results and Discussions $\quad 107$

6.1 Assumptions and specifications ............... 107

6.2 Flowchart ......................... 109

6.3 Code verification . . . . . . . . . . . . . . . 112

6.4 Performance in the case of perfect channel estimation . . . . . . . . . 114

6.4.1 Effect of having multirate users . . . . . . . . . . . 114

6.4.2 Performance of best and worst users . . . . . . . . . . 119

6.4.3 Effect of user cancellation order . . . . . . . . . . . 123

6.4.4 Effect of assignment of Gold sequences to users . . . . . . . 125

6.4.5 Effect of the method of implementation of VPG . . . . . . 129

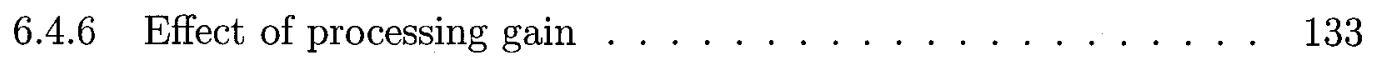


6.4.7 Effect of extremely disparate rates . . . . . . . . . 134

6.5 Performance in the case of imperfect channel estimation . . . . . . . 136

6.5.1 Effect of having multirate users . . . . . . . . . . 136

6.5.2 Effect of user cancellation order . . . . . . . . . . 136

6.6 Performance of the joint channel estimator multiuser detector . . . . 142

6.6.1 Performance of the channel estimator . . . . . . . . . . . . . 142

6.6.2 Performance of the joint channel estimator multiuser detector 144

7 Conclusions and Future Work 153

7.1 Conclusion and summary . . . . . . . . . . . . . . 153

7.2 Future work .......................... 156

$\begin{array}{ll}\text { A Computational Complexity } & 159\end{array}$

$\begin{array}{ll}\text { B DBSIC BER Performance Analysis } & 167\end{array}$

$\begin{array}{ll}\text { C Conventional SIC BER Performance Analysis } & 171\end{array}$

D AME Performance Analysis of DBSIC for AWGN Channels $\quad 174$

E AME Performance Analysis of DBSIC for Rayleigh Channels 182

$\begin{array}{ll}\text { References } & 213\end{array}$ 


\section{List of Figures}

3.1 Block diagram for a single-rate DS-CDMA system . . . . . . . . . 38

3.2 Virtual users' signals for the simulated multirate CDMA system . . . 39

3.3 Multipath Fading Channel Model . . . . . . . . . . . . . 50

3.4 Block diagram for the VPG multirate CDMA system . . . . . . . . 52

3.5 RAKE receiver with MRC . . . . . . . . . . . . . 54

3.6 DBSIC structure . . . . . . . . . . . . . . . 62

4.1 Channel Estimator structure . . . . . . . . . . . . . 86

5.1 Performance of DBSIC over 2-path Rayleigh fading channel with RAKE, perfect channel estimate, $G_{1}=32 \ldots \ldots$. . . . . . . . 91

5.2 Asymptotic Multiuser Efficiency for user 2 over the AWGN Channel,

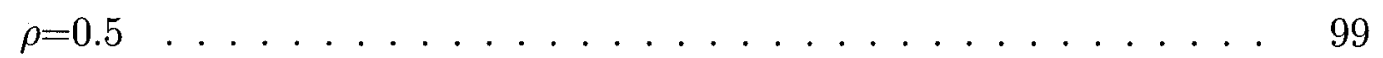

5.3 Asymptotic Multiuser Efficiency for user 2 over the AWGN Channel,

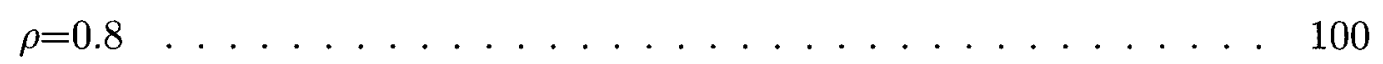

5.4 Asymptotic Multiuser Efficiency for user 2 over the AWGN Channel,

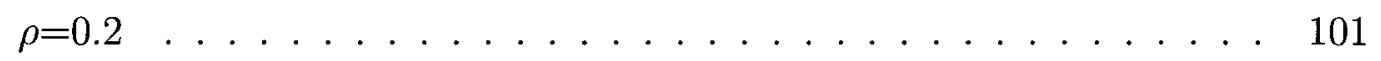

5.5 Asymptotic Multiuser Efficiency for user 1 over a Rayleigh Fading

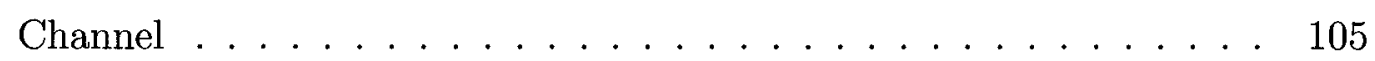


5.6 Asymptotic Multiuser Efficiency for user 2 over a Rayleigh Fading

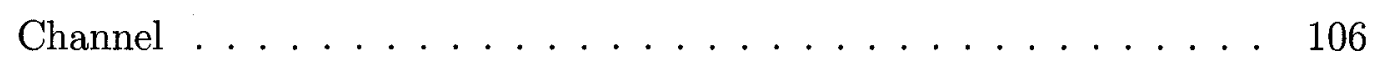

6.1 Flowchart for the simulation code with no channel estimation scheme implemented . . . . . . . . . . . . . . . .

6.2 Flowchart for the simulation code with channel estimation scheme im-

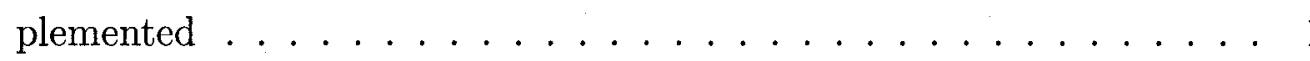

6.3 BER performance under single-path AWGN channel, 10 single-rate users with processing gain of $32 \ldots \ldots \ldots$

6.4 BER performance under single-path Rayleigh Fading channel, 10 singlerate users with processing gain of $32 \ldots \ldots . \ldots . \ldots 114$

6.5 Performance over 2-path Rayleigh fading channel with RAKE, perfect channel estimation, $K_{1}=10, G_{1}=32 \ldots \ldots \ldots$

6.6 Performance over 2-path Rayleigh fading channel with RAKE, perfect channel estimation, $K_{1}=7, K_{2}=3, G_{1}=32 \ldots \ldots \ldots$

6.7 Performance over 2-path Rayleigh fading channel with RAKE, perfect channel estimation, $K_{1}=5, K_{2}=3, K_{3}=2, G_{1}=32 \ldots \ldots$

6.8 Performance over 2-path Rayleigh fading channel with RAKE, perfect channel estimation, single and multi-rates, $G_{1}=32 \ldots \ldots$

6.9 Performance over 2-path Rayleigh fading channel with RAKE, perfect channel estimation, $K_{1}=10, G_{1}=32 \ldots \ldots \ldots$

6.10 Performance over 2-path Rayleigh fading channel with RAKE, perfect channel estimation, $K_{1}=7, K_{2}=3, G_{1}=32 \ldots \ldots$

6.11 Performance over 2-path Rayleigh fading channel with RAKE, perfect channel estimation, $K_{1}=5, K_{2}=3, K_{3}=2, G_{1}=32 \ldots \ldots$ 
6.12 Performance over 2-path Rayleigh fading channel with RAKE, perfect channel estimation, $K_{1}=4, K_{2}=3, K_{3}=2, K_{4}=1, G_{1}=32 \ldots$.

6.13 Performance over 2-path Rayleigh fading channel with RAKE, perfect channel estimation, $K_{1}=3, K_{2}=2, G_{1}=32$ (MF outputs/random user cancellation order $) \ldots \ldots \ldots \ldots$

6.14 Performance over 2-path Rayleigh fading channel with RAKE, perfect channel estimation, $K_{1}=10, G_{1}=32$, different set of Gold sequences

6.15 Performance over 2-path Rayleigh fading channel with RAKE, perfect channel estimation, $K_{1}=7, K_{2}=3, G_{1}=32$, different set of Gold

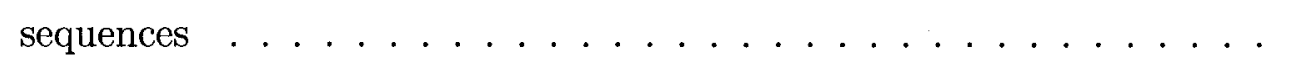

6.16 Performance over 2-path Rayleigh fading channel with RAKE, perfect channel estimation, $K_{1}=5, K_{2}=3, K_{3}=2, G_{1}=32$, different set of Gold sequences . . . . . . . . . . . . . . . . .

6.17 Performance over 2-path Rayleigh fading channel with RAKE, perfect channel estimation, $K_{1}=4, K_{2}=3, K_{3}=2, K_{4}=1, G_{1}=32$, different set of Gold sequences . . . . . . . . . . . . . . . .

6.18 Performance over 2-path Rayleigh fading channel with RAKE, perfect channel estimation, $K_{1}=10, G_{1}=32$, virtual users signatures sequences generated with the 1 st method . . . . . . . . . .

6.19 Performance over 2-path Rayleigh fading channel with RAKE, perfect channel estimation, $K_{1}=7, K_{2}=3, G_{1}=32$, virtual users signatures sequences generated with the 1 st method . . . . . . . . . .

6.20 Performance over 2-path Rayleigh fading channel with RAKE, perfect channel estimation, $K_{1}=5, K_{2}=3, K_{3}=2, G_{1}=32$, virtual users signatures sequences generated with the 1 st method . . . . . . . . 
6.21 Performance over 2-path Rayleigh fading channel with RAKE, perfect channel estimation, $K_{1}=4, K_{2}=3, K_{3}=2, K_{4}=1, G_{1}=32$, virtual users signatures sequences generated with the 1 st method . . . . . .

6.22 Performance over 2-path Rayleigh fading channel with RAKE, perfect channel estimation, $K_{1}=17, K_{2}=3, G_{1}=64 \ldots \ldots \ldots$

6.23 Performance over 2-path Rayleigh fading channel with RAKE, perfect channel estimation, $K_{1}=1, K_{30}=1, G_{1}=64 \ldots \ldots$

6.24 Performance over 2-path Rayleigh fading channel with RAKE, $12.5 \%$ channel estimate error, single and multi-rates, $G_{1}=32 \ldots \ldots$

6.25 BER performance over 2-path Rayleigh fading channel with RAKE, $12.5 \%$ channel estimate error, $K_{1}=3, K_{2}=2, G_{1}=32$ (MF outputs/random user cancellation order) . . . . . . . . .

6.26 BER performance over 2-path Rayleigh fading channel with RAKE, $25 \%$ channel estimate error, $K_{1}=3, K_{2}=2, G_{1}=32$ (MF outputs/random user cancellation order) . . . . . . . . . . .

6.27 BER performance over 2-path Rayleigh Fading channel with RAKE, Decorrelating detector, $K_{1}=3, K_{2}=2, G_{1}=32 \ldots \ldots \ldots$

6.28 BER performance over 2-path Rayleigh Fading channel with RAKE, conventional SIC detector (MF outputs/random user cancellation ordering), $K_{1}=3, K_{2}=2, G_{1}=32 \ldots \ldots \ldots$

6.29 BER performance over 2-path Rayleigh Fading channel with RAKE, DBSIC detector (MF outputs/random ordering), $K_{1}=3, K_{2}=2$, $G_{1}=32 \ldots \ldots \ldots \ldots \ldots \ldots$

6.30 Normalized MSE of channel fading coefficients of the virtual users over 2-path Rayleigh fading channel with RAKE, $G_{1}=32 \ldots \ldots$ 
6.31 Performance over 2-path Rayleigh fading channel with RAKE, joint channel estimation and multiuser detection, $K_{1}=10, G_{1}=32 \ldots 144$

6.32 Performance over 2-path Rayleigh fading channel with RAKE, joint channel estimation and multiuser detection, $K_{1}=7, K_{2}=3, G_{1}=32$

6.33 Performance over 2-path Rayleigh fading channel with RAKE, joint channel estimate and multiuser detection, $K_{1}=5, K_{2}=3, K_{3}=2$, $G_{1}=32 \ldots \ldots \ldots \ldots \ldots \ldots \ldots \ldots \ldots \ldots \ldots \ldots \ldots \ldots$

6.34 Performance over 2-path Rayleigh fading channel with RAKE, joint channel estimate and multiuser detection, $K_{1}=4, K_{2}=3, K_{3}=2$, $K_{4}=1, G_{1}=32 \ldots \ldots \ldots \ldots$

6.35 Performance of DBSIC over 2-path Rayleigh fading channel with RAKE, (joint channel estimation and multiuser detection versus multiuser detection with perfect channel estimation), single and multi-rate, $G_{1}=32148$

6.36 Performance over 2-path Rayleigh fading channel with RAKE, (joint channel estimation and multiuser detection versus multiuser detection with perfect channel estimation), $K_{1}=10, G_{1}=32 \ldots \ldots$

6.37 Performance over 2-path Rayleigh fading channel with RAKE, (joint channel estimation and multiuser detection versus multiuser detection with perfect channel estimation), $K_{1}=7, K_{2}=3, G_{1}=32 \ldots \ldots$

6.38 Performance over 2-path Rayleigh fading channel with RAKE, (joint channel estimation and multiuser detection versus multiuser detection with perfect channel estimation), $K_{1}=5, K_{2}=3, K_{3}=2, G_{1}=32$.

6.39 Performance over 2-path Rayleigh fading channel with RAKE, (joint channel estimation and multiuser detection versus multiuser detection with perfect channel estimation), $K_{1}=4, K_{2}=3, K_{3}=2, K_{4}=1$, $G_{1}=32 \ldots \ldots \ldots \ldots \ldots \ldots \ldots \ldots \ldots \ldots \ldots \ldots \ldots \ldots \ldots$ 


\section{List of Tables}

3.1 Preferred Pairs for Gold Sequences . . . . . . . . . . . . . . . . 42

3.2 Signature Sequences for a 3-low-rate and 2-high-rate system . . . . . 46

5.1 Simulated physical users rates . . . . . . . . . . . . . 90

6.1 Simulated physical users rates . . . . . . . . . . . . . 115 


\section{List of Abbreviations}

\begin{tabular}{|c|c|}
\hline $3 \mathrm{G}$ & Third generation \\
\hline $4 \mathrm{G}$ & Fourth generation \\
\hline AME & Asymptotic Multiuser Efficiency \\
\hline AR & Auto Regressive \\
\hline AWGN & Additive White Gaussian Noise \\
\hline BER & Bit-Error-Rate \\
\hline BPSK & Binary Phase Shift Keying \\
\hline CDMA & Code Division Multiple Access \\
\hline CG & Conjugate Gradient \\
\hline CIR & Channel Impulse Response \\
\hline CSI & Channel state information \\
\hline DBSIC & Decorrelator Based Successive Interference Cancellation \\
\hline DDFB & Decorrelating Decision Feedback \\
\hline DF & Decision Feedback \\
\hline DHIC & Decorrelating Based Hybrid Interference Cancellation \\
\hline DS & Direct-Sequence \\
\hline EGC & Equal-Gain-Combining \\
\hline $\mathrm{EM}$ & Expectation Maximization \\
\hline FEC & Forward Error Correction \\
\hline & Frequency Hopped \\
\hline
\end{tabular}


GWPIC Group-Wise Parallel Interference Cancellation

GWSIC Group-Wise Successive Interference Cancellation

GWSSUS Gaussian Wide Sense Stationary Uncorrelated Scattering

HD Hard Decision

HIC Hybrid Interference Cancellation

HRD High Rate Decorrelator

IC Interference Cancellation

ISI InterSymbol Interference

LFSR Linear Feedback Shift Register

LMMSE Linear Minimum-Mean-Square-Error

LMS Least-Mean-Square

LOS Line-of-Sight

LRD Low Rate Decorrelator

MAI Multiple Access Interference

MAME Multirate Asymptotic Multiuser Efficiency

MAP Maximum A-Posteriori Probability

MC Multi-code

MCH Multi-Channel

MDM Modulation-Division Multiplexing

MF Matched Filter

MGA Multivariate Gaussian Approximation

ML $\quad$ Maximum Likelihood

MMD Multi-Modulation

MMSE Minimum-Mean-Square-Error

MNFR Multirate Near-Far Resistance 
MPG Multiple Processing Gain

MRC Maximum Ratio Combining

NFR Near-Far Resistance

PCG Preconditioned Conjugate Gradient

PIC Parallel Interference Cancellation

PN Pseudo-Random

PPIC Partial Parallel Interference cancellation

RSL Recursive Least Squares

SC Selection Combining

SD Steepest Descent

SDC Selection Diversity Combining

SER Symbol Error Rate

SIC Successive Interference Cancellation

SINR Signal-to-Interference-Plus-Noise Ratio

SISO Soft-In-Soft-Out

SNR Signal-to-noise ratio

SS Spread Spectrum

TD Total Degradation

TDL Tapped Delay Line

TH Time-Hopping

UMTS Universal Mobile Telecommunication System

VCR Variable Chip Rate

VPG Variable Processing Gain

VSL Variable Spreading Length 


\section{List of Symbols}

page where

symbol

$\underline{\text { Symbol }}$

$\underline{\text { Explanation }}$

is defined

$\alpha[l] \quad l^{\text {th }}$ path gain (general multipath fading channel assuming time-invariance over one block of $N$ symbols) $\ldots \ldots \ldots \ldots \ldots \ldots \ldots \ldots \ldots .50$

$\alpha_{c, k}^{(j)}[l] \quad l^{\text {th }}$ path gain for the $j^{\text {th }}$ virtual user derived from user $k$ of class $c$, $\alpha_{c, k}^{(j)}[l]=\alpha_{c, k}^{\left(j^{\prime}\right)}[l]$, for all $j, j^{\prime}=1, \ldots, c \ldots \ldots \ldots \ldots \ldots \ldots \ldots \ldots \ldots \ldots \ldots$

$\alpha_{m}[l] \quad l^{t h}$ path channel fading gain of the $m^{\text {th }}$ virtual user (assuming slow

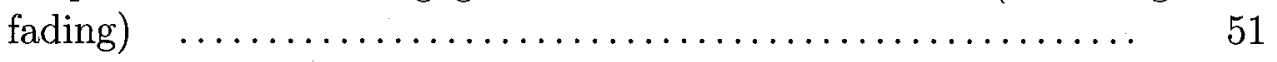

$\vec{\alpha}_{m} \quad=\left[\alpha_{m}[1], \alpha_{m}[2], \cdots, \alpha_{m}[L]\right]^{T} \quad \ldots \ldots \ldots \ldots \ldots \ldots \ldots \ldots \ldots \ldots \ldots \ldots \ldots \ldots \ldots \ldots \ldots$

$\alpha_{c, k}^{(j)}[i, l] \quad l^{t h}$ path gain of the $j^{\text {th }}$ virtual user derived from the $k^{\text {th }}$ physical user of class $c$ for the duration of the $i^{\text {th }}$ transmitted symbol $\quad \ldots . \quad 76$

$\alpha_{m}[i, l] \quad l^{\text {th }}$ path channel fading gain of the $m^{\text {th }}$ virtual user during the $i^{t h}$

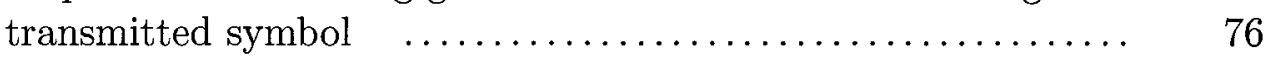

$\vec{\alpha}_{m}[i] \quad=\left[\alpha_{m}[i, 1], \alpha_{m}[i, 2], \cdots, \alpha_{m}[i, L]\right]^{T} \quad \ldots \ldots \ldots \ldots \ldots \ldots \ldots \ldots \ldots \ldots \ldots \ldots$

$\vec{\alpha}[i] \quad=\left[\vec{\alpha}_{1}^{T}[i], \vec{\alpha}_{2}^{T}[i], \cdots, \vec{\alpha}_{K_{v}}^{T}[i]\right]^{T} \quad \ldots \ldots \ldots \ldots \ldots \ldots \ldots \ldots \ldots \ldots \ldots \ldots \ldots \ldots \ldots \ldots$

$\vec{\alpha}^{J} \quad=\left[\vec{\alpha}^{T}[1], \vec{\alpha}^{T}[2], \cdots, \vec{\alpha}^{T}[N]\right]^{T} \ldots \ldots \ldots \ldots \ldots \ldots \ldots \ldots \ldots \ldots \ldots \ldots \ldots \ldots$

$\alpha_{k} \quad$ Path gain of the $k^{t h}$ physical user (synchronous scenario,

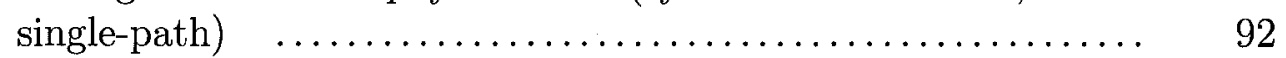

$a_{c, k}[i] \quad$ Value of $i^{\text {th }}$ chip for the $k^{\text {th }}$ physical user of class $c \quad \ldots \ldots \ldots .43$ 
$b_{c, k}^{(j)}[i] \quad i^{\text {th }}$ transmitted symbol for the $j^{\text {th }}$ virtual user derived from user $k$ in class $c, j=1, \cdots, c \quad \ldots \ldots \ldots \ldots \ldots \ldots \ldots \ldots \ldots \ldots \ldots \ldots \ldots . \quad 46$

$b_{m}[i] \quad i^{\text {th }}$ transmitted symbol for the $m^{\text {th }}$ virtual user $\quad \ldots \ldots \ldots \ldots . . \ldots 1$

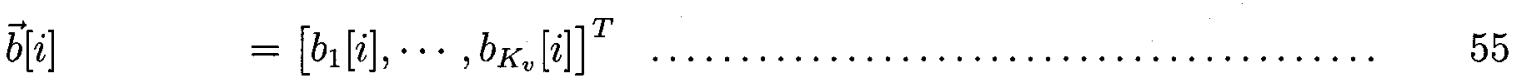

see also $\quad \ldots \ldots \ldots \ldots \ldots \ldots \ldots \ldots \ldots \ldots \ldots \ldots \ldots \ldots \ldots \ldots \ldots, \quad 72$

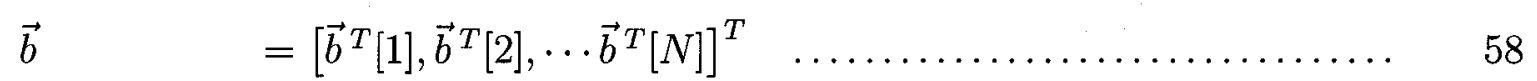

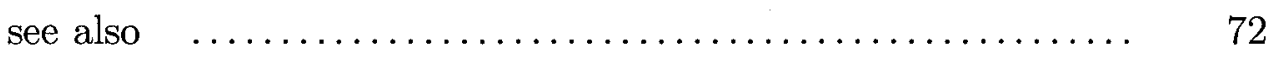

$\vec{b}_{n e w} \quad$ Vector obtained by reordering $\vec{b}$ according to the reordering of $\vec{y} \quad 58$

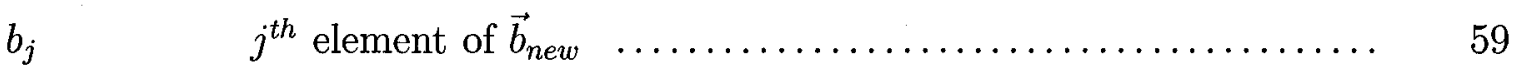

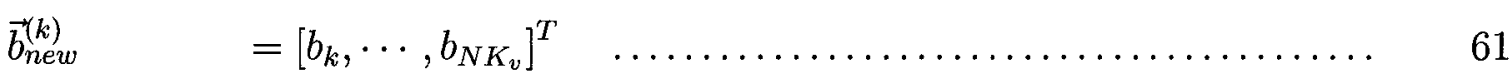

$\mathbf{b}_{\mathbf{m}}[\mathbf{i}] \quad=\operatorname{diag}\left(b_{m}[i], b_{m}[i], \cdots, b_{m}[i]\right) \quad \ldots \ldots \ldots \ldots \ldots \ldots \ldots \ldots \ldots . \ldots \ldots$

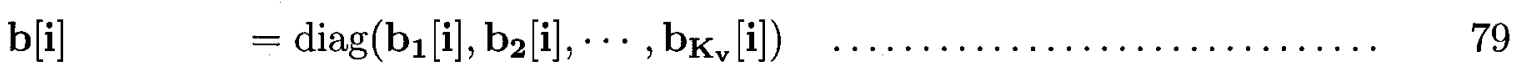

$\overline{\mathbf{b}} \quad=\operatorname{diag}(\mathbf{b}[\mathbf{1}], \mathbf{b}[\mathbf{2}], \cdots, \mathbf{b}[\mathbf{N}]) \quad \ldots \ldots \ldots \ldots \ldots \ldots \ldots \ldots \ldots \ldots$

$b_{k}^{\mathrm{s}} \quad$ Transmitted symbol of the $k^{\text {th }}$ physical user (synchronous

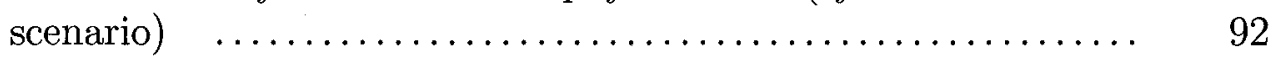

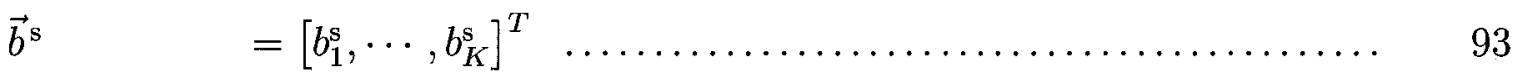

$\vec{b}_{\text {new }}^{\text {s }} \quad$ Vector obtained by reordering $\vec{b}^{\mathrm{s}}$ according to the reordering of

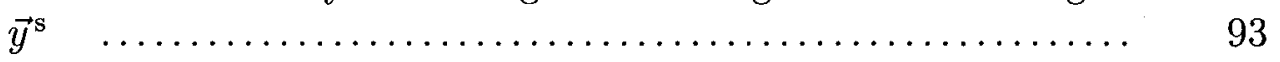

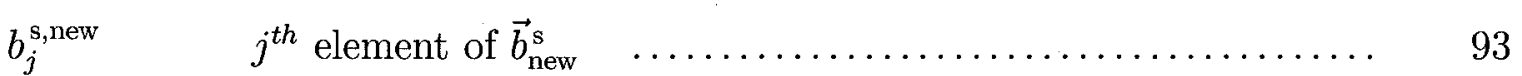

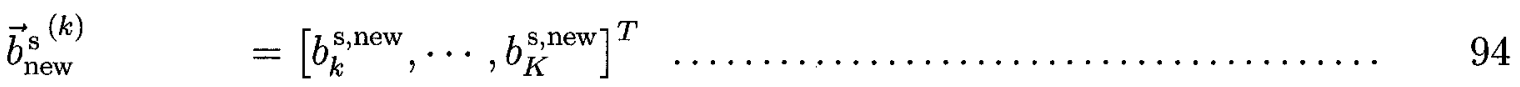

$C \quad$ Total number of user classes, users in one class have the same transmission rate $\ldots \ldots \ldots \ldots \ldots \ldots \ldots \ldots \ldots \ldots \ldots \ldots . \ldots \ldots, \quad 37$

$\begin{array}{lll}c & \text { Class of a user, each user in class } c \text { has transmission rate } R_{c} \quad . \quad 37\end{array}$

$C_{m}(t) \quad$ Effective signature sequence for the $m^{\text {th }}$ virtual user,

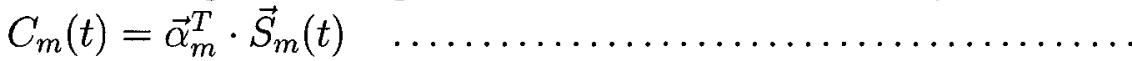

$C_{k}^{s}(t) \quad$ Effective signature sequence for the $k^{\text {th }}$ physical user (synchronous case,

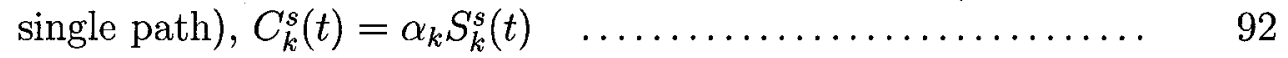


$\eta$

$e_{k}(\sigma)$

$E_{c, k}^{(j)} / c$

$E_{m} / c$

$\mathbf{E}$

$\overline{\mathrm{E}}$

$\mathbf{E}_{\text {new }}$

$\mathbf{E}_{\mathbf{m}}$

$\mathbf{E}^{\mathbf{J}}$

$\overline{\mathbf{E}}^{\mathbf{J}}$

$E_{k}^{s}$

$\boldsymbol{E}_{\mathrm{s}}$

$\boldsymbol{E}_{\text {s,new }}$

$\epsilon_{k}$

$E_{b}$

$\mathbf{F}$

$G_{c}$

$K$

$K_{c}$

$K_{v}$
Asymptotic Multiuser Efficiency $\ldots \ldots \ldots \ldots \ldots \ldots \ldots \ldots . . . \ldots 5$

Effective energy of the $k^{\text {th }}$ user $\ldots \ldots \ldots \ldots \ldots \ldots \ldots \ldots \ldots \ldots$

Received signal energy for $j^{\text {th }}$ virtual user derived from user $k$ of class $c$

Received signal energy of the $m^{t h}$ virtual user

(assuming $m^{\text {th }}$ virtual user is of class $c$ )

$=\operatorname{diag}\left(\sqrt{E_{1}}, \cdots, \sqrt{E_{K_{v}}}\right)$

see also

$=\operatorname{diag}(\mathbf{E}, \mathbf{E}, \cdots, \mathbf{E})$

see also

Matrix obtained by reordering $\overline{\mathbf{E}}$ according to the reordering of $\vec{y} \quad 58$

$=\operatorname{diag}\left(\sqrt{E_{m}}, \sqrt{E_{m}}, \cdots, \sqrt{E_{m}}\right)$

$=\operatorname{diag}\left(\mathbf{E}_{\mathbf{1}}, \mathbf{E}_{\mathbf{2}}, \cdots, \mathbf{E}_{\mathbf{K}_{\mathbf{v}}}\right)$

$=\operatorname{diag}\left(\mathbf{E}^{\mathbf{J}}, \mathbf{E}^{\mathbf{J}}, \cdots, \mathbf{E}^{\mathbf{J}}\right)$

Signal energy of the $k^{\text {th }}$ physical user (synchronous case) $\quad \ldots \quad 92$

$=\operatorname{diag}\left(\sqrt{E_{1}^{s}}, \cdots, \sqrt{E_{K}^{s}}\right)$

Matrix obtained by reordering $\boldsymbol{E}_{\mathrm{s}}$ according to the reordering of $\vec{y}$

$k k^{\text {th }}$ element of the diagonal matrix $\overline{\mathbf{E}}_{\text {new }}$

Average energy per bit

see also

Lower triangular matrix such that $\overline{\mathbf{Z}}=\mathbf{F}^{H} \mathbf{F}$

Processing gain for class $c$ users

Total number of users in the system for all classes, $K=\sum_{c=1}^{C} K_{c}$

Number of users in class $c$

Total number of virtual users in the system for all classes, $K_{v}=\sum_{c=1}^{C} c K_{c}$ 
$L \quad$ Number of paths of the channel $\quad \ldots \ldots \ldots \ldots \ldots \ldots \ldots \ldots, 50$

$N \quad$ Number of symbols transmitted for class 1 (slowest rate) users ( $=$ number of bits if binary modulation is used) $\quad \ldots \ldots \ldots \ldots . \quad 45$

$n(t) \quad$ Complex Gaussian noise random process with zero mean and power

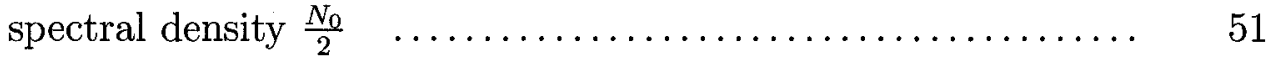

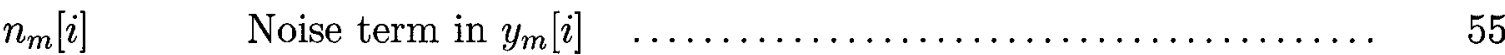

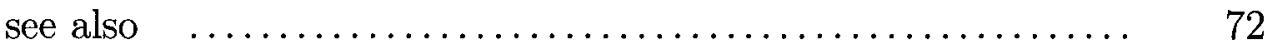

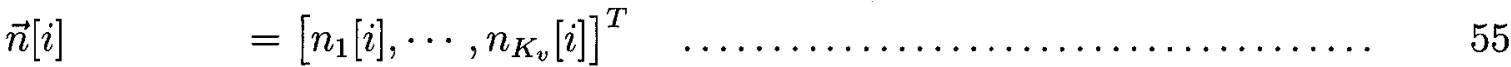

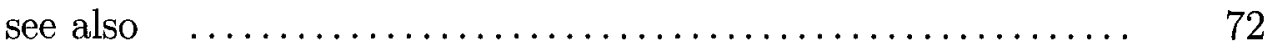

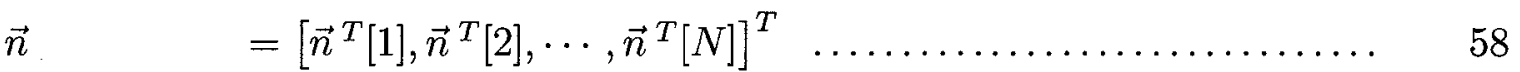

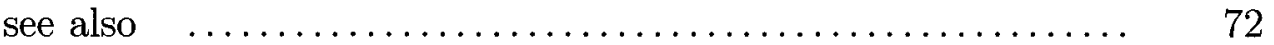

$\vec{n}_{n e w} \quad$ Vector obtained by reordering $\vec{n}$ according to the reordering of $\vec{y} \quad 58$

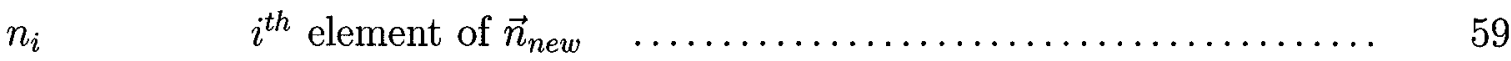

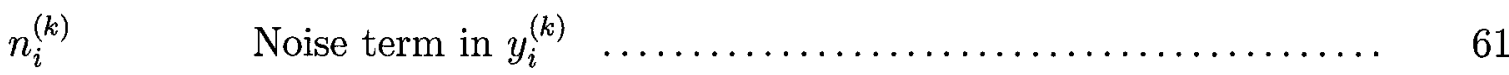

$\vec{n}_{n e w}^{(k)} \quad=\left[n_{k}^{(k)}, \ldots, n_{N K_{v}}^{(k)}\right]^{T} \ldots \ldots \ldots \ldots \ldots \ldots \ldots \ldots \ldots \ldots \ldots \ldots \ldots \ldots \ldots \ldots \ldots \ldots \ldots \ldots$

$\vec{n}_{f} \quad$ Noise term in $\vec{y}_{f} \quad \ldots \ldots \ldots \ldots \ldots \ldots \ldots \ldots \ldots \ldots \ldots \ldots \ldots \ldots \ldots, 64$

$N_{0} \quad$ Noise power $\quad \ldots \ldots \ldots \ldots \ldots \ldots \ldots \ldots \ldots \ldots \ldots \ldots \ldots \ldots \ldots \ldots, 51$

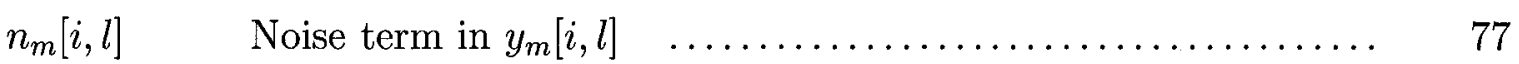

$n_{k}^{\mathbf{s}} \quad$ Noise term in $y_{k}^{\mathbf{s}} \quad \ldots \ldots \ldots \ldots \ldots \ldots \ldots \ldots \ldots \ldots \ldots \ldots, 93$

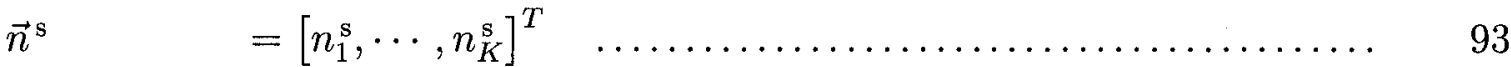

$\vec{n}_{\text {new }}^{\mathrm{s}} \quad$ Vector obtained by reordering $\vec{n}^{\mathrm{s}}$ according to the reordering of

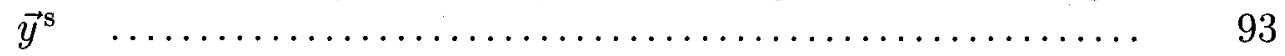

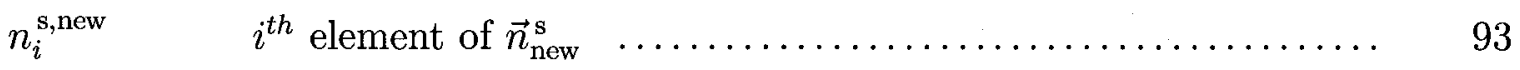

$\vec{n}_{\text {new }}^{\mathrm{s}(k)} \quad=\left[n_{k}^{\mathrm{s}(k)}, \ldots, n_{K}^{\mathrm{s}(k)}\right]^{T} \ldots \ldots \ldots \ldots \ldots \ldots \ldots \ldots \ldots \ldots \ldots \ldots \ldots \ldots \ldots \ldots \ldots$

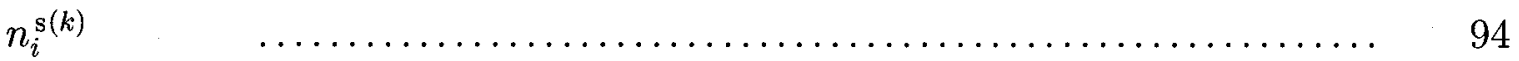

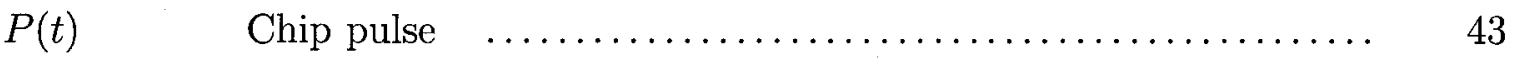


$P_{R}(r) \quad$ Rayleigh probability distribution $\quad \ldots \ldots \ldots \ldots \ldots \ldots \ldots \ldots . \ldots \ldots$

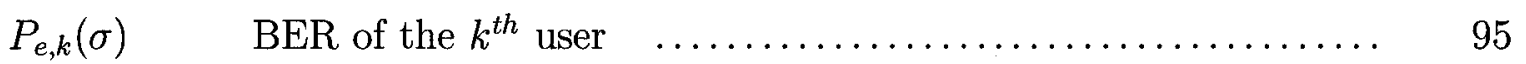

$R_{c} \quad$ Transmission rate for class $c$ users $\quad \ldots \ldots \ldots \ldots \ldots \ldots \ldots \ldots$

$R \quad$ Slowest data rate (base rate), $R=R_{1} \ldots \ldots \ldots \ldots \ldots \ldots \ldots$

$r(t) \quad$ Baseband received signal $\quad \ldots \ldots \ldots \ldots \ldots \ldots \ldots \ldots \ldots \ldots \ldots \ldots \ldots \ldots$

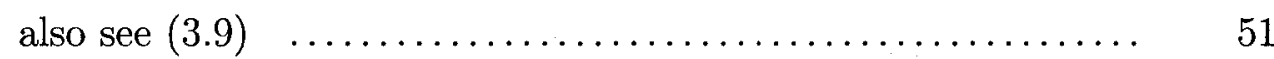

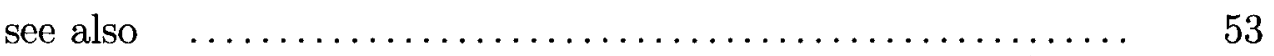

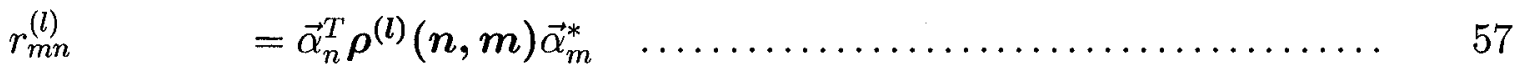

$\mathbf{R}(l) \quad$ Matrix with $(m n)^{t h}$ element $r_{m n}^{(l)} \quad \ldots \ldots \ldots \ldots \ldots \ldots \ldots \ldots \ldots \ldots \ldots \ldots$

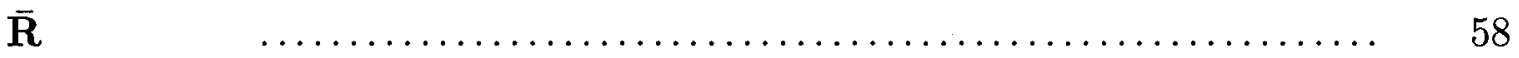

$\overline{\mathbf{R}}_{\text {new }} \quad$ Matrix obtained by reordering $\overline{\mathbf{R}}$ according to the reordering of $\vec{y} \quad 58$

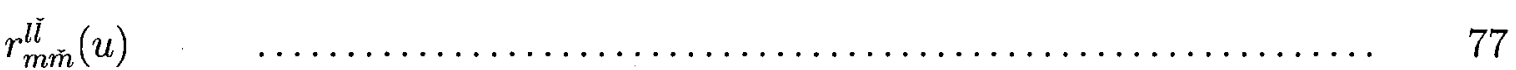

$\mathbf{R}_{\mathbf{m n}}(\mathbf{u}) \quad$ Matrix with $l \check{l}$ element $r_{m n}^{l \check{l}}(u) \quad \ldots \ldots \ldots \ldots \ldots \ldots \ldots \ldots \ldots \ldots \ldots \ldots \ldots \ldots$

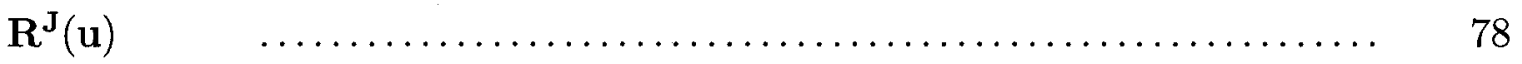

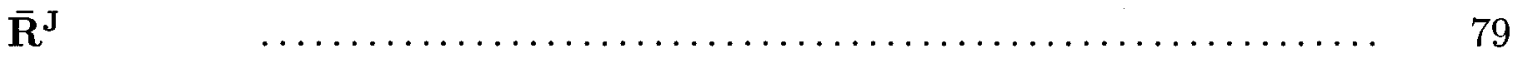

$\boldsymbol{R}_{\mathrm{s}} \quad$ Correlation matrix of the user spreading sequences (synchronous case), matrix with $m n^{\text {th }}$ element $\rho_{n m}^{\mathrm{s}} \quad \ldots \ldots \ldots \ldots \ldots \ldots \ldots . . \ldots 3$

$\boldsymbol{R}_{\mathrm{s}, \text { new }} \quad$ Matrix obtained by reordering $\boldsymbol{R}_{\mathrm{s}}$ according to the reordering of

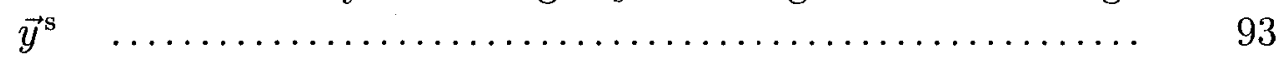

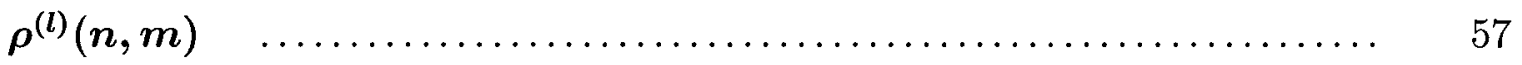

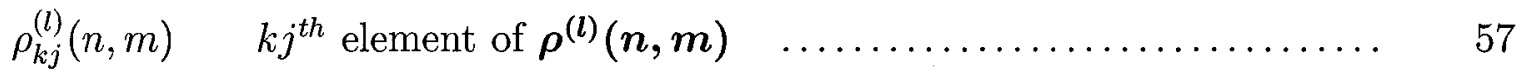

$\rho_{n m}^{\mathrm{s}} \quad$ Cross correlation of the effective spreading sequences of user $n$ and user $m$ (synchronous case) $\quad \ldots \ldots \ldots \ldots \ldots \ldots \ldots \ldots . . \ldots 3$

$\rho \quad$ Cross-correlation of the user spreading sequences (synchronous

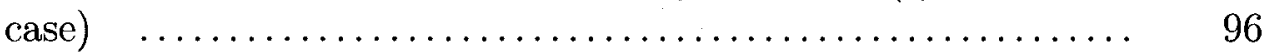

$S_{c, k}(t) \quad$ Signature sequence (waveform) of the $k^{\text {th }}$ physical user of class $c$ (first

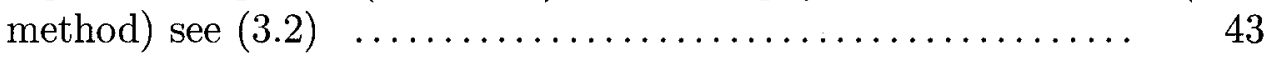


$S_{c, k}(t) \quad$ Signature sequence (waveform) of the $k^{\text {th }}$ physical user of class $c$ (second

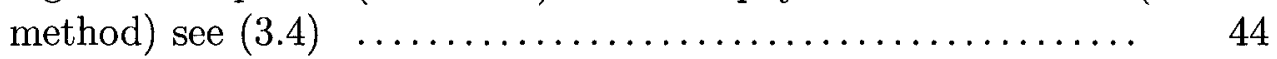

$S_{c, k}^{(j)}(t) \quad$ Signature sequence of the $j^{t h}$ virtual user corresponding to the $k^{t h}$ physical user of class $c$ (first method) see (3.3) $\ldots \ldots \ldots \ldots \ldots$

$S_{c, k}^{(j)}(t) \quad$ Signature sequence of the $j^{\text {th }}$ virtual user corresponding to the $k^{t h}$ physical user of class $c$ (second method) see (3.5) $\ldots \ldots \ldots \ldots \quad 45$

$S_{m}(t) \quad$ Signature code of the $m^{t h}$ virtual user $\quad \ldots \ldots \ldots \ldots \ldots \ldots \ldots \ldots$

$\vec{S}_{m}(t) \quad=\left[S_{m}(t), S_{m}\left(t-T_{c h}\right), \cdots, S_{m}\left(t-(L-1) T_{c h}\right)\right]^{T} \quad \ldots \ldots \ldots \ldots . \quad 52$

$S_{k}^{s}(t) \quad$ Signature sequence of the $k^{t h}$ physical user (synchronous case) 92

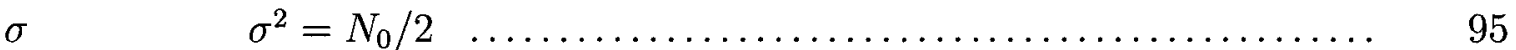

$\tau_{m} \quad$ Delay of the $m^{t h}$ virtual user $\quad \ldots \ldots \ldots \ldots \ldots \ldots \ldots \ldots \ldots \ldots \ldots \ldots \ldots \ldots$

$\tau_{c, k}^{(j)} \quad$ Delay of the $j^{\text {th }}$ virtual user derived from user $k$ of class $c, \tau_{c, k}^{(j)}=\tau_{c, k}^{\left(j^{\prime}\right)}$ for all $j, j^{\prime}=1, \ldots, c \quad \ldots \ldots \ldots \ldots \ldots \ldots \ldots \ldots \ldots \ldots \ldots \ldots$

$T_{s} \quad$ Duration of one symbol (or one bit if binary modulation is used) of the slowest rate users $T_{s}=T_{1, s} \quad \ldots \ldots \ldots \ldots \ldots \ldots \ldots \ldots \ldots \ldots \ldots \ldots \ldots \ldots, 38$

$T_{c h} \quad$ Delay of the channel tapped-delay line model, is also equal to the chip

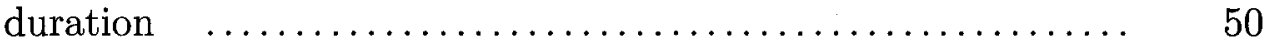

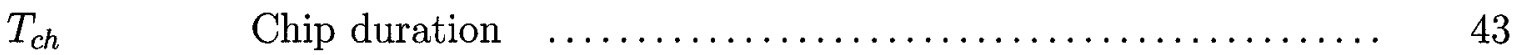

$T_{c, s} \quad$ Symbol interval for class $c$ users $\ldots \ldots \ldots \ldots \ldots \ldots \ldots \ldots \ldots$

$\mathbf{V}^{(k)} \quad=\left\{\overline{\mathbf{Z}}^{(\mathbf{k})}\right\}^{-1} \quad \ldots \ldots \ldots \ldots \ldots \ldots \ldots \ldots \ldots \ldots \ldots \ldots \ldots \ldots \ldots \ldots \ldots \ldots \ldots \ldots \ldots$

$v_{i j}^{(k)}$

$i j^{\text {th }}$ element of $\mathbf{V}^{(k)}$

$x_{c, k}^{(j)}(t) \quad$ Continuous-time baseband-equivalent signal transmitted by $j^{\text {th }}$ virtual user derived from user $k$ of class $c \quad \ldots \ldots \ldots \ldots \ldots \ldots \ldots . . \ldots \ldots$

$y_{m}[i] \quad$ Output of a RAKE matched filter, matched to the user effective signature $C_{m}\left(t-\tau_{m}-(i-1) T_{s}\right)$, sampled at $T_{s} \ldots \ldots \ldots \ldots \quad 55$

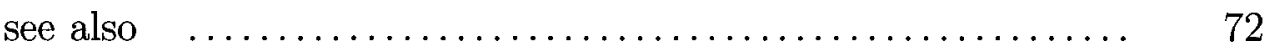

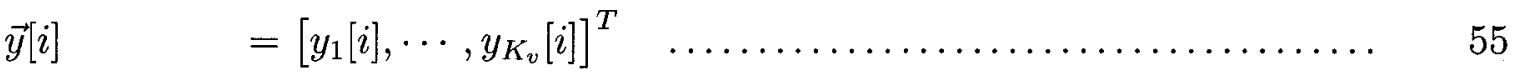

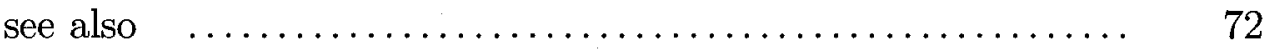


$\vec{y}$

Output of a bank of $K_{v}$ RAKE matched filters, matched to the effective signature sequences of each user, sampled at $T_{s}$ see (3.15) $\ldots \quad 57$

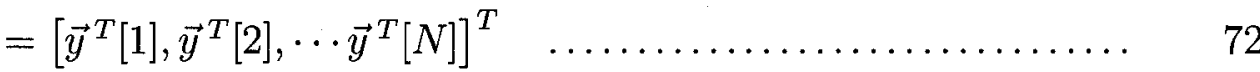

$y_{i}^{\mathrm{MF}}$

$i^{\text {th }}$ element of $\vec{y}$

$\vec{y}_{\text {new }}$

Vector obtained by reordering $\vec{y}$ so that its elements $y_{i}$ are listed in decreasing order

$y_{i}$

$i^{\text {th }}$ element of $\vec{y}_{n e w}$ see $(3.17)$

$=y_{i}$

see $(3.23)$

$=\left[y_{k}^{(k)}, \cdots, y_{N K_{v}}^{(k)}\right]^{T}$

$\vec{y}_{f}$

Output obtained when $\mathbf{F}^{H^{-1}}$ (noise whitening filter) is applied to $\vec{y}_{n e w}$

$y_{f, k}$

$k^{\text {th }}$ element of $\vec{y}_{f}$

$y_{m}[i, l]$

Output of a RAKE matched filter, matched to the user spreading signature $S_{m}\left(t-\tau_{m}-(i-1) T_{s}-(l-1) T_{c h}\right)$, sampled at $T_{s}$

$=\left[y_{m}[i, 1], y_{m}[i, 2], \cdots, y_{m}[i, L]\right]^{T}$

$=\left[\vec{y}_{1}^{T}[i], \vec{y}_{2}^{T}[i], \cdots, \vec{y}_{K_{v}}^{T}[i]\right]^{T}$

$=\left[\vec{y}^{J}[1]^{T}, \vec{y}^{J}[2]^{T}, \cdots, \vec{y}^{J}[N]^{T}\right]^{T}$

$y_{k}^{\mathrm{s}}$

Output of a matched filter, matched to the user effective spreading sequence $C_{k}^{s}(t)$, sampled at $T_{s}$ (synchronous case) $\ldots \ldots \ldots \ldots .92$

$=\left[y_{1}^{\mathrm{s}}, \cdots, y_{K}^{\mathrm{s}}\right]^{T}$

Vector obtained by reordering $\vec{y}^{\mathrm{s}}$ so that its elements $y_{i}^{\mathrm{s}}$,new are listed in decreasing order 
$\overline{\mathbf{Z}}$

$=\overline{\mathbf{R}}_{\text {new }} \overline{\mathbf{E}}_{\text {new }}$

$i j^{\text {th }}$ element of $\overline{\mathbf{Z}}$

$\overline{\mathbf{Z}}^{(\mathbf{1})}$

$=\overline{\mathbf{Z}}$

Truncated matrix $\overline{\mathbf{Z}}$

$\mathrm{Z}_{\mathrm{s}}$

$=\boldsymbol{R}_{\mathrm{s}, \text { new }} \boldsymbol{E}_{\mathrm{s}, \text { new }}$ 


\section{Chapter 1}

\section{Introduction}

\subsection{Thesis Objectives}

The new generation of wireless communication systems (such as advanced cellular phone, personal digital assistant and others) is growing rapidly with the increasing demand for systems capacity and broadband multimedia services including data, voice, images and video transmission. All these services require different amount of information to be transmitted. The users will be transmitting their information signals using different data rates and their performance requirements will vary from application to application. Hence, it is desirable to develop wireless systems that operate at multiple data rates in order to accommodate the various demands from users as well as addressing possibly the need of services with different customer costs. The present third generation (3G) systems can provide a maximum data rate of $2 \mathrm{Mbits} / \mathrm{s}$ for indoor environment which is much less than that needed for the currently used multimedia applications. The fourth generation $(4 \mathrm{G})$ wireless networks demand even

higher data rate up to $100 \mathrm{Mbits} / \mathrm{s}$ [1]. Code division multiple access (CDMA) is a promising technique for multiuser wireless communication where multiple users share 
the same communication channel. However, as all users share the same frequency band, different users in CDMA do interferer with each other unless the transmissions from all users are perfectly synchronized and the corresponding spreading codes used are strictly orthogonal. In wireless channels, these two conditions are difficult to achieve. Furthermore, delay spread might be significant in such channels leading to intersymbol interference (ISI) as well. In CDMA systems the interferences from other users corrupt the received signal of the desired user and the interference is termed as multiple access interference (MAI). Multirate multiuser detection becomes a challenge and a hot topic of research for CDMA systems due to the severe ISI, MAI and near-far effects under multipath fading channels.

The conventional single-user detector treats MAI as additional noise and may even suffer from the near-far problem [2] which occurs whenever the cross correlation between the spreading sequences of the user of interest and the signal from a strong interferer is larger than the correlation with the signal from the desired user. The near-far problem increases the probability of detection error of the desired signal due to the interference from the stronger signal. Performance of CDMA systems is limited by MAI and deteriorates especially in the asynchronous transmission case due to the near-far effect. The channel utilization is correspondingly low. Multiuser detection techniques are effective technologies to combat MAI in CDMA communication and improve the signal-to-interference-plus-noise ratio (SINR) and systems capacity. CDMA systems also suffer from ISI under multipath channels. In order to overcome ISI, multiuser detectors are usually designed to estimate an entire packet of data. Various types of multiuser detectors have been developed in the last decade [2].

There are several ways to design a multirate system. In the simplest way, the transmission of a symbol at slow rates in a multirate CDMA system can be treated as a repeated transmission of the symbol at the fastest rate in the system [3] but this is 
practical when only a few rates are to be supported. More conventional strategies are as follows. Multi-code (MC) or multi-channel (MCH) [3] allocate several parallel communication channels to higher rate users. Modulation-division multiplexing (MDM) [4] or multi-modulation (MMD) [3] use higher-order modulations for high rate users. Variable Processing Gain (VPG) [5], Multi Processing gain [3], Multiple Processing Gain (MPG) [6] or Variable Spreading Length (VSL) [7] maintain a fixed chip rate but assign smaller processing gains and symbol durations for high rate users. Variable chip rate (VCR) maintains a fixed processing gain but assigns different chip rate.

Since MCH is often outperformed by VPG and noting that VCR is usually more complex practically due to the fact that the receiver must be synchronized to its particular code rate and additional frequency planning is required due to the unequal bandwidth spreading of different users [8], we will focus on VPG. In this work we study multiuser detection techniques for uplink multirate CDMA systems with VPG scheme under multipath Rayleigh fading channels with additive white Gaussian noise (AWGN).

Among possible multiuser schemes, interference cancellation (IC) multiuser receivers such as successive interference cancellation (SIC) or parallel interference cancellation (PIC) receivers provide some advantages. Of relatively low complexity and suitable for hardware implementation compared to other multiuser schemes, they can be easily combined with other techniques such as channel estimation or error correction coding [5]. The principle of SIC over a frequency selective channel is to estimate the transmitted data of each user from the matched filter outputs (correlators) of a conventional maximal ratio combining RAKE receiver where the MAI associated with the previously detected ("strongest") users has been regenerated and subtracted. The process is performed successively such that the user with the largest average power (indexed as user 1) is canceled first, and the user with the second largest power (in- 
dexed as user 2) is canceled next. In other words, to detect the transmitted signal of user $k$, the SIC detector treats the signals of the weaker users $k+1, \cdots, K$ as additive noise and assumes the decisions of users $1, \cdots, k-1$ are correct. The strongest user is not known beforehand, and is usually detected from the strength of the correlations of each of the users chip sequence with the received signal (initialization using conventional matched filter detection). The correlation values (obtained from the conventional bank of correlators) are passed on to a selector which ranks all users according to their estimated average power obtained from the correlators outputs and selects the corresponding user for decoding and cancellation. Since the principle of SIC is based on regeneration of the MAI using the detected signals, if the MAI estimate of the signal of previous users is poor in the cancellations, the estimation errors propagate to all users and may yield to performance degradation $[9,10]$.

Considering that generally the decorrelating detector can achieve better bit-errorrate (BER) performance than the conventional single-user matched filter (MF) detector especially at high signal-to-noise ratio (SNR), this thesis proposes a new asynchronous multirate detection scheme, namely the decorrelator based SIC (DBSIC) receiver. The DBSIC scheme is similar to the conventional SIC scheme, except that at each stage of the SIC receiver, a decorrelator on top of conventional MF detector is employed to detect that user's signal. Besides its improved performance compared to the conventional MF detector under high SNR conditions, the decorrelating detector is near-far resistant and has no requirement of power control implementation due to the fact that it is independent of the interfering power (amplitude). Therefore by implementing a decorrelator in the early stages of DBSIC fewer decision errors are obtained, reducing consequently the error propagations to the following stages. Using a Gaussian approximation approach, a theoretical BER performance analysis has been done. Results obtained from the theoretical performance analysis have been 
compared with the results obtained from numerical simulations. DBSIC effectiveness to combat MAI has also been evaluated in terms of its asymptotic multiuser efficiency (AME) over AWGN and Rayleigh channels.

The first proposed implementation of DBSIC like many multiuser detectors requires knowledge of the multipath fading channel. In practice the channel information is estimated and thus may be subject to channel estimation errors. In the case of imperfect channel estimation, it has been observed while both commonly used multiuser detectors and DBSIC suffer degradation in performance, DBSIC still outperforms the other detection schemes. Since the performance of a wireless communication system depends mainly on its ability to obtain an accurate estimation of the time-varying multipath channel, a joint channel estimation and multiuser detection technique is proposed in this thesis as well. With a reasonable computational complexity, the proposed adaptive MAI-resistant multiuser channel estimation scheme is based on the combination of decision-directed and data-aided approaches and works jointly with the multiuser detection technique. The effectiveness of this joint channel estimation and multiuser detection scheme is supported by results in terms of channel estimates MSE and BER performance.

\subsection{Thesis Contributions}

The main contributions achieved are as follows:

- A new DBSIC scheme for multirate multiuser detection is presented with the introduction of an architecture, which is moderately more complex, but offers a superior performance compared to the conventional SIC, decorrelator or decorrelating DF receivers (see section 3.3). DBSIC is similar to the conventional SIC detector except that a decorrelator is utilized on top of the conventional 
single-user MF detector to determine the signal estimate. Due to the fact that the decorrelating detector outperforms the conventional MF detector particularly at high SNR and the SIC detector usually starts the detection from the users with the strongest receiving power or signal amplitude, DBSIC can have a much more accurate signal estimate at each stage. Therefore, DBSIC combines the advantages of SIC, and linear decorrelating to combat multiple access interference.

- A semi-analytical BER performance analysis of DBSIC is presented and DBSIC performance is compared to conventional SIC (see section 5.1).

- The analytical AME performance analysis is derived for DBSIC and compared with the AME of other multiuser detectors over AWGN and Rayleigh fading channels (see section 5.2).

- Various multiuser detection schemes including conventional single-user MF, decorrelating, minimum-mean-square-error (MMSE), SIC, PIC and decorrelating DF are studied and implemented for a multirate asynchronous CDMA system. Their performances under both perfect and imperfect channel estimation are compared with that of DBSIC receiver scheme (see section 6.4 and section 6.5).

- A new joint channel estimation and multiuser detection technique using DBSIC principles is proposed for the multirate CDMA system. The proposed adaptive MAI-resistant multiuser channel estimation scheme is based on the combination of decision-directed and data-aided approaches and works jointly with the multiuser detection technique (see section 4.2). Its effectiveness is demonstrated in terms of channel estimate MSE and BER performance (see section 6.6).

- To reduce complexity of the asynchronous decorrelating detector, one method 
consists of passing the matched filter bank output through a noise whitening filter. A simple method to implement the ideal noise whitening filter by providing a novel adaptive algorithm that performs the cross-correlation matrices factorization is introduced (see section 3.6). The introduced algorithm not only implements the ideal noise whitening filter, but also reduces the computational complexity that is related to the matrix inversion for the asynchronous decorrelating receiver.

- A modified cancellation structure for PIC scheme is proposed where the latest estimates from other users instead of those obtained from previous stage are fed back directly in the current cancellation stage (see section 3.7). The main advantage of this structure is that it does not increase the complexity of the scheme while taking advantage of the results from newest detected users.

\subsection{Thesis Organization}

- Chapter 2 provides a literature review on detection techniques and performance analysis for single-rate and multirate asynchronous CDMA systems, as well as channel estimation methods.

- Chapter 3 presents the CDMA systems, channel models and the various multirate asynchronous multiuser receiver structures investigated and proposed in this thesis including the DBSIC structure.

- Chapter 4 reviews channel estimation methods and presents the proposed new joint channel estimation and multiuser detection technique.

- Chapter 5 describes the multiuser schemes theoretical performance analysis considering both BER and AME. 
- Chapter 6 discusses the simulation results under various scenarios.

- Chapter 7 summarizes the thesis and proposes future works. 


\section{Chapter 2}

\section{Literature Review}

\subsection{Literature review on multiuser detection}

\subsubsection{Introduction: single-rate versus multi-rate CDMA systems}

CDMA is a multiple access technique where multiple users share the same communication channel. This can be achieved using spread spectrum techniques. Spread spectrum (SS) is a means of transmission where the signals are spread over a large frequency band and transmitting the spread signal with a low power per unit bandwidth. The bandwidth occupied by the signals is much larger than that necessary to send the information. The SS modulation techniques were originally developed for use in the military and intelligence communications systems to provide antijam and low probability of interception, since the large bandwidth of SS signals makes them more immune to narrowband interferences.

There are several ways of generating a spread spectrum signal: frequency hopped (FH), time-hopping (TH) and direct-sequence (DS) spread spectrum signals. In THSS the data is divided into frames which are divided into time intervals. The data in burst is hopped over the frames by utilizing code sequences/spreading codes. In a 
FH-CDMA spread spectrum system, in each signaling interval the transmitted signal of one user occupies one or several frequencies slots out of the total available channel bandwidth. The frequencies slot(s) of each user change in each signaling interval according to a pseudo random pattern. The spreading code is used to generate a pattern of frequency hops (e.g. carrier frequencies hops) which avoids other users. The spreading code in this case does not directly modulate the data-modulated carrier but instead controls the sequence of carrier frequencies. FHSS is an avoidance technique where the reduction in interference occurs because the signal is made to avoid the interference a large fraction of the time.

In a DS-CDMA system with Binary Phase Shift Keying (BPSK), the modulated signal of one user (narrowband data) is obtained by multiplying its data signal with a pseudo-random wideband signal (usually referred to as spreading sequence or chip sequence) and then passing the output through a BPSK modulator. Different signature sequence/spreading sequence can be assigned to different users in a DS-CDMA system so that the users can share the same communication channel. DS-SS technique results in a processing gain, usually defined as $G_{c}=\frac{\text { transmitted signal bandwidth }}{\text { original data bandwidth }}$. The higher the processing gain the better the system performance as the lower the interference. The capacity of a DS-CDMA system is not subject to a hard limit (like time division multiple access) and increasing the number of users will reduce the received signal-to-interference ratio and performance.

In this thesis we will focus on DS-CDMA systems. MAI and ISI are two major factors limiting the performance and hence the capacity of a DS-CDMA system. The most effective approach to suppress the MAI and ISI for a CDMA system is multiuser detection, in which all signals from all users are detected jointly and simultaneously by the receiver. Depending on the number of data rates in the system, a CDMA system can be classified as single-rate or multi-rate. 
Single-rate CDMA system: The single-rate system is the simplest and most common CDMA system and has been addressed in literature and research for decades. There is only one data-rate in the system, in other word, all users in the system will transmit the information bit at the same data rate and all users will have the same spreading gain overall. The commonly used multiuser detection schemes discussed in section 2.1.2 are well developed and can be used for the single-rate CDMA system directly.

Multi-rate CDMA system: There are several ways to design a multirate system. In the simplest way, the transmission of a symbol at slow rates in a multirate CDMA system can be treated as a repeated transmission of the symbol at the fastest rate in the system. And repeated transmission of symbols can be viewed as a simple binary repetition code [3] or a form of time-diversity signaling. Therefore, the multirate system can be reduced to a single rate encoded system. However, this is practical when only a few rates are to be supported. Using this approach, [11] investigates decorrelating, MMSE and PIC schemes for multirate systems under an AWGN channel. More conventional strategies consist of the following methods $[3,4]$ :

1. $\mathrm{MC}$ or $\mathrm{MCH}[3]$ : design a standard CDMA system and allocate several parallel communication channels to higher rate users. Several spreading codes are used in parallel to increase the data rate. Multiple receivers are needed to demodulate data. Multiple parallel data channels (spreading codes) will create multiple access type of interference.

2. MDM [4] or MMD [3]: use a higher-order modulation (i.e. use a modulation alphabet with more symbols for a high rate user). For example a low-rate user could use QPSK modulation, and a user with four times higher data rate could 
use 16 QAM. All users have the same symbol rate and processing gains, but different bit rates.

3. VPG [3], MPG [6] or VSL [7]: the processing gain is altered so that all signals are modulated to the same bandwidth (independently of the bit rate). In other words, the chip rate is maintained fixed and different data rates are accommodated with the assignment of signature sequences of different lengths. The principle is to choose smaller processing gains and symbol durations for higher rate users than for lower rate users. Thus high rate users can transmit more symbols in a given time. Such scheme is a form of time division multiplexing. In order to maintain the same link quality (e.g., same $E_{b} / N_{0}$, where $E_{b}$ is the bit energy) for both low data rate and high data rate users, the transmitted power of a high data rate user (low processing gain) is higher than the power of a low bit rate user (high processing gain), which causes worse near-far effect. In other words, the signal of a high data rate user has larger amplitude than that of a low data rate user so that the former has same bit energy as the latter. To increase the data rate only a piece of spreading code is used in spreading, resulting in smaller processing gain. Low processing gains will introduce ISI like in TDMA systems but the ISI can be combated to some extent by using spreading codes longer than one symbol interval.

4. VCR [12]: variable data rates are supported for various multimedia applications by varying the chip rate in accordance with the assigned data rate while maintaining the processing gain fixed. 


\subsubsection{Single-rate multiuser systems}

Due to the inherent presence of MAI in CDMA systems, multiuser detection has been one of the major challenges to implement CDMA wireless systems and has received considerable attention in recent years. Maximum likelihood (ML) detector (optimum), suboptimum: conventional (single-user), decorrelating (completely nearfar resistant), MMSE detectors and other more complex multiuser detectors such as blind adaptive (no training symbols required), SIC, PIC, multistage SIC and PIC, and Decision Feedback (DF) detectors were developed to enhance the performance of CDMA systems by reducing MAI [13][14].

Among the existing multiuser detectors, the optimum detector over an AWGN channel can completely eliminate MAI [15] while minimizing the probability of error [2], but has the most computational complexity with arithmetic operations growing exponentially with the number of users which is not practically suitable for implementation. It consists of a bank of $K$-matched filters plus samplers, or $K$ correlators, one for each user code sequence followed by a decision device ( $K$ is the number of users). The detector (decision device) following the matched filters or correlators computes the $2^{K}$ correlation metrics and selects the signal that is corresponding to the largest correlation metric. Such operation might be implemented using a Viterbi forward dynamic programming algorithm. The optimum receiver requires a priori knowledge of the signal amplitudes and phases for maximum symbol decision at the receiver [9].

The conventional single-user detector is the simplest suboptimum multiuser detector, and is also based on matched filtering techniques. It treats MAI as additional noise and assumes that the combined noise and interference is white and Gaussian. The exact knowledge of the users' signature sequences and the transmitted signal timing for each user are needed to implement this detector. It has a complexity that 
grows linearly with the number of users but still has serious limitation due to the near-far problem.

The decorrelating detector [13] is a linear multiuser detector with reduced complexity compared to the optimal multiuser detector which makes use of the structure of MAI to improve performance. The cross-correlations of the user signature sequences are assumed to be known. The matched filter outputs are multiplied by the inverse of the cross-correlation matrix, which contains all the signature cross-correlations. The new set of decision variables decouples MAI significantly. The linear decorrelating detector achieves optimal demodulation when the channel is noiseless, and in comparison with the conventional single user detector is near-far resistant at high SNR. The disadvantage of the decorrelating detector is that it enhances the noise after the linear transformation which results in significant performance degradation at low SNR. In fact, at low SNR, the MAI is of the same order as the background noise and the performance of the decorrelating detector may be worse than that of the conventional single-user detector [16] [17]. The asynchronous decorrelating detector also involves the inversion of a cross-correlation matrix but is more complex to implement, due to the fact that the inverse does not always exist, or even if it does, the matrix dimension is proportional to the product of the number of users and the sequence length (which may be very large), thereby leading in detection delay. Several variations of the decorrelating receiver for asynchronous CDMA have been proposed to overcome the problem. For example a truncated decorrelating detector is studied in $[6,18]$. A recursive linear detection algorithm that needs the inversion of only a $K \times K$ matrix at each step was proposed in [19]. Since the noise term in the matched filter bank output is no longer white due to the despreading process [2], another approach consists to pass the matched filter bank output by an ideal noise whitening filter before making the decision. Various near ideal or approximate 
noise whitening filters have been proposed or examined in [20]-[23]. In section 3.6 a novel iterative method is introduced to implement the ideal noise whitening filter. The proposed method has a low level complexity while achieving the performance of the classical detectors for asynchronous transmissions. In order to reduce complexity, another approach is to combine the decorrelating detector with diversity combining techniques. For example, a one-shot linear decorrelating detector with pre-selection diversity combining (SDC) and post maximal ratio combining was introduced in [24]. A modified decorrelator based on a two-stage scheme is presented in [25] which can achieve significant performance improvement. A hard decision is made at first stage for the decorrelator outputs with high reliability. Rest of the outputs are passed to the second stage with a conditional quantization algorithm for a final decision to make. [26] analyzes the effects of the nonlinear distortions on the performance of the decorrelating detector in DS-CDMA downlink systems and derives theoretically the symbol error rate (SER) and the total degradation (TD) in AWGN and frequency-flat Rayleigh fading channels by assuming and motivating the Gaussian distribution of the nonlinear distortion noise.

The MMSE detector also performs a linear transformation on a block of data from the correlator or matched filter outputs, but it minimizes the mean square error between the matched filter output and the source data, which can improve the detector's performance since it incorporates some SNR information in the detection algorithm [27]-[29]. It maximizes the SINR in comparison to the other linear detectors. An iterative IC scheme is considered in [30] to improve the speed of convergence of the adaptive MMSE receiver for the reverse-link of a DS-CDMA system. The proposed iterative algorithm makes use of the available knowledge of all users training sequences at the base-station receiver to jointly cancel MAI and adapts to the MMSE optimum filter taps using the combined adaptive MMSE/PIC receiver. 
Blind adaptive detectors require no training symbols. The only required system parameter to be known is the signature sequence of the user of interest. The most popular blind adaptive detectors decompose the linear multiuser detection filter response into two orthogonal components, where one of them equals the signature sequence of the desired user. Such blind adaptive detector can effectively suppress ISI and MAI in multiuser CDMA systems using the adaptive algorithm without requiring training symbols [31]. A couple of new blind adaptive detectors have been proposed in recent years. For example, an improved blind adaptive MMSE receiver for fast fading DS-CDMA channels was provided in [32] by designing estimators for the interference correlation matrix.

Another class of multiuser detectors is based on the principle of IC using an approach such as SIC or PIC. In the first stage of a SIC detector, all users are ranked according to their signal strengths, the strongest user is detected and the MAI caused by that user is regenerated and subtracted from the matched filter output of a conventional maximal ratio combining RAKE receiver corresponding to the second strongest user. That second strongest user is then detected. This process is repeated until all users have been detected, where at each stage the MAI caused by the already detected users is regenerated and subtracted. In other words, at each stage of SIC we detect one user at a time, treating the signals of the weaker users $k, \cdots, K$ as additive noise and assumes the decisions of users $1, \cdots, k-1$ are correct [33]. The conventional SIC is normally initialized by a conventional or decorrelating or any other linear multiuser receiver to detect the strongest user and after that the conventional MF detector is employed for the subsequent stage for decision. Initially, uncoded transmission was considered and hard decisions were used at each stage [33]. The obtained detector is easy to implement but may suffer from performance degradation if the MAI estimate of the signal of previous users is poor in the cancellations since the estimation errors 
propagate to all users. This may occur for example in case of channel estimation errors, or in heavily loaded CDMA systems where the performance of the conventional receiver is poor. Specifically the SIC detector may suffer from two nonideal cancellation effects: i) residual cancellation errors whereby the user detected in a stage is not completely canceled in that stage and ii) partial signal cancellation effects whereby part of a users signal is removed prior to the stage in which it is to be detected.

Several approaches can be used to improve the performance of SIC, including the use of multistages. As shown in [34] the BER performance can be improved by changing the users' order after each cancellation in SIC receivers with one or two stages of cancellation over AWGN and Rayleigh fading channels. A modified multistage SIC structure, which is shown to be resistant to the near-far effect, is presented in [35] under multipath channel for Universal Mobile Telecommunication System (UMTS). [36] shows that multistage SIC with an infinite number of stages can be employed to implement asynchronous conventional decorrelating and MMSE detectors over AWGN channels. It is shown that in practice only a few stages are needed for multistage SIC to achieve the same BER as MMSE and decorrelating detectors over AWGN channels. A few approaches were recently reported that attempt to improve signal reconstruction in SIC with either more accurate amplitude estimates or more reliable symbol decisions. For example more reliable symbol decisions (and hence less error propagation) can be obtained by the use of soft decisions and multistage IC [37][38], also referred to as soft or partial IC. An improved SIC receiver with a new softdecision rule which employs amplitude averaging and is robust to timing errors is presented in [39]. The amplitude averaging combines the linear and hard decision rules. SIC can also be combined with channel coding and soft-in-soft-out (SISO) decoding (see for example [40] and references herein). [41] investigates the impact of the number of multipath components on the system capacity employing SIC with 
optimum power control and finds that it works well only with a limited number of multipath components.

Unlike the SIC detector, the PIC detector regenerates and subtracts all the MAI for each user in parallel. The multistage PIC detector employs multiple stages to detect the signals and subtract the interference, where more interference is removed as the detection proceeds to subsequent stages [42]. The decorrelator is implemented in the first stage for the multistage PIC detector to provide reliable initial decisions for later cancellation stages. The multistage PIC detector presented in [42] was designed for AWGN channels and was later extended to multipath fading channels in [43]. [44] compares the performance of a two-stage PIC scheme and the decorrelating detector over 2-path Rayleigh fading channels. It shows that PIC achieves better performance in known channels than the decorrelating receiver but is more sensitive to channel estimation error, and suffers from BER saturation when the number of users is large. [45] presents an accurate performance analysis method of asynchronous CDMA systems employing multistage PIC detection. Similar to SIC, performance of multistage PIC can be improved by the use of partial PIC (PPIC) [46]. A new PPIC scheme that is a combination of soft and hard PIC detectors whose performance is superior to that of the other famous suboptimal detectors is proposed in [47]. An estimation of parameters starts after a few stages of soft IC and hard IC is performed in the subsequent stages.

Another type of IC scheme is the decorrelating DF detector or zero-forcing DF detector [13]. By applying a noise whitening filter to the matched filter bank outputs, a white noise model is obtained where the users have been partially decorrelated. SIC procedure is then applied. [48] proposes an orthogonal DF detector that contains an orthogonal feedforward filter and a decision feedback structure. It is computationally more efficient in dynamically updating the feedforward and feedback filter pairs than 
the decorrelating DF detector.

Various receivers combining several of the multiuser detection techniques previously presented have been proposed in the literature. For example, a new decorrelating based hybrid interference cancellation (DHIC) which combines SIC, PPIC and decorrelator was proposed in [49] for a synchronous CDMA system. This detector first selects a group of strongest users and applies the decorrelating technique to obtain the reliable channel estimation and PPIC input. The second step is to perform the PPIC scheme to detect all the transmitted signals. The results show that DHIC has better performance than conventional MF, PPIC and SIC detector. A MMSE/SIC hybrid multiuser receiver is presented in [50]. The users are also first divided into groups based on the power level they are in. MMSE is employed to detect users within each group while SIC is still used for the interference cancellation among different groups. [51] investigates partial successive interference cancellation in hybrid DS/FH spread-spectrum multiple-access (SSMA) systems which can achieve the same good BER performance as that of a full interference cancellation. A hybrid interference cancellation scheme combining PIC and SIC is proposed in [52] and the performance is compared with other hybrid cancellation schemes. [53] introduces a hybrid interference cancellation receiver for MC-CDMA system and the results show the advantage of the hybrid scheme over PIC and SIC. Another HIC with short detection delay and good performance is introduced in [54].

Reference [55] presents simulation comparison results for five multiuser receivers, namely the decorrelating receiver, the MMSE receiver, the multistage PIC receiver, the SIC receiver, and the decorrelating DF receiver. It compares the BER performance in AWGN, Rayleigh fading, and "Near-Far" channels.

All the previously cited references consider single-rate CDMA systems. However, it is also desirable to develop wireless systems that operate at multiple data rates in 
order to accommodate various demands from users.

\subsubsection{Multi-rate multiuser systems}

Receivers for multirate CDMA systems are based on the same principles as singlerate CDMA multiuser schemes such as the conventional single-user, ML, decorrelating, MMSE, SIC and multistage PIC approaches.

Using a single-user detector, based on matched filtering (for AWGN channels) and RAKE detecting techniques with maximum ratio combining (MRC) or selection combining (SC) (for multipath channels) [3] studies MDM, VPG, MC and VC multirate DS-CDMA schemes for both AWGN and multipath Rayleigh fading channels. Using the classical Gaussian interference approximation to model the interference from the interfering users, [3] shows that VPG and MC can achieve almost the same performance but the performance for high rate users of the MDM strategy is degraded compared to VPG and MC. Using a Gaussian approximation, exact error probability was also obtained. Using the true interference distribution instead of the classical Gaussian approximation, [56] shows that the difference in error performance in addition to SINR between dual-rate MC and VPG systems strongly depends on distributions of multiple access and multipath interferences, but is likely to be negligible in practical scenarios. The exact error probability was obtained in [57] for both AWGN and multipath fading channels, and shown to be accurate when the number of interference is large or SNR is low.

The performance of ML based detection is studied in [7] using either MC or VPG/VSL and the two methods are compared based on their AME. The AME is an indication of the performance degradation of a receiver due to MAI as the additive channel noise decreases for a fixed set of received powers for all users. Based on the AME, it was shown that over an AWGN channel the VSL/VPG outperforms MC for 
the detection of high rate data users, and yields similar performance as MC for low rate data users. This favors the use of VSL especially in a multistage approach as it also has a lower complexity than MC systems. These results were extended to 2-path Rayleigh fading channels in [58]. Performance was evaluated based on bounds to the minimum probability of error as well as the AME. [59] also evaluates the performance of VSL and MC in terms of AME and near-far resistance (NFR). The results show that MC-CDMA has a similar performance as that of VSL-CDMA employing low-rate detection in terms of multirate AME (MAME) and multirate NFR (MNFR).

The performance of a multirate CDMA decorrelating detector is presented in terms of BER and AME in [4] using either the MC and VPG approaches. Numerical results show no significant differences in performance between the two schemes. A high rate decorrelator (HRD) for a dual rate synchronous VPG DS-CDMA system corrupted by AWGN is proposed in [8]. It is shown how the dual rate system is equivalent to a single rate system, in which each low rate user is modeled as a high rate user in each bit interval of the high rate user. The classical decorrelator of [60] [61] applied to this equivalent single rate system is referred to as the low rate decorrelator (LRD). It is shown in [61] that that the asymptotic efficiency of the LRD is greater than or equal to the HRD. But the simulation results show that the HRD performs nearly as well as the LRD when the signature sequences have good correlation properties, while being of lower complexity and reducing the bit processing delay of high rate users. The probabilities of error of the LRD and HRD are analyzed in [62], and it is shown that the LRD outperforms the HRD. A sliding-window decorrelator with performance better than the HRD, while being less complex than the LRD is also proposed. Finally, [62] proposes a decorrelating decision feedback detector that exploits further the characteristics of multirate systems. The proposed scheme reduces the demodulation delay for high rate users and provides better detection performance for low 
rate users than the LRD when the desired user and the interfering users have comparable energies. A truncated window decorrelator for an asynchronous VPG multirate CDMA system is proposed in [63] where a user is decoded by employing sliding finite length observation windows that extend over a sufficient number of its bits. Bounds on the asymptotic efficiency of the proposed receiver which converge rapidly to the asymptotic efficiency of the infinite window decorrelator when the length of the observation window increases are also developed in [63]. [64] presents a generalization of the conventional decorrelating detector, designed to deal with the multirate UMTS signal by taking into account the two-layered UMTS spreading codes to characterize MAI. A variable processing gain serial pseudo-noise modulation multirate CDMA system (which combines MC and VPG) is introduced in [65]. The uncoded BERs with and without decorrelating detection are derived analytically and simulated on a multipath fading channel. Performance is also presented for a system that uses turbo codes. A power control algorithm, not requiring a measurement of uplink channel gain but employing stochastic approximation methods, is proposed in [66] for a multirate decorrelator. The algorithm converges to an optimal power in the mean square sense when the user bit has nonzero asymptotic efficiency. [67] applies the MDM, MC and VSL schemes for decorrelating detection in multi-rate DS-CDMA systems by jointly considering signal constellations and MAI. It demonstrates that MDM outperforms MC and VSL in the scenario of large number of active users for high-rate transmission. A multirate decorrelating detector employing VPG scheme is analyzed in [68] for a multicarrier DS-CDMA mobile multimedia system and it is shown to achieve almost the same performance as the single-rate decorrelating detector.

The structure of the optimal linear MMSE receiver for an asynchronous multirate CDMA system is derived in [69] by converting the original multirate system into an equivalent single-rate multiuser system. A lower complexity suboptimal receiver 
that exploits the inherent cyclostationarity of the DS-CDMA is then proposed. Such scheme is a function of the cyclic statistics of the signals. The proposed framework applies to both VPG and VC multirate CDMA asynchronous systems for either single or multi-carrier scenarios. The MMSE receiver for a multirate receiver with both VPG and $\mathrm{VC}$ is studied in [70] over an asynchronous multipath Rayleigh fading channel. Different feed-forward/feed-back architectures are proposed for the implementation of the MMSE receiver in the multirate systems. A VPG multirate CDMA system where users are allowed to change rates while calls are connected, is considered in [71] for a flat fading channel. In that situation, classical adaptive MMSE receivers may result in performance degradation during the transient after the rate change. To avoid this performance degradation [71] investigates an adaptive MMSE receiver (based on least-mean-square (LMS)) that allows updating the weight vectors for all rates simultaneously. [72] proposes an improved adaptive MMSE multiuser detection algorithm to solve the problem that characteristics of MAI change periodically in multirate transmission and spreading method of W-CDMA systems. [73] investigates the impact of channel estimation error on the performance of decorrelating and MMSE receivers and the influence is equivalent for both receivers in terms of SNR and BER. A MMSE receiver structure with antenna arrays employing various multi-rate schemes is presented in [74]. The results suggest that the selection of the multirate scheme in implementation depends on the power consumptions, the hardware complexity of the receiver and operating scenario.

Blind detection techniques for VPG or MC multirate CDMA systems, which combine concepts of the code-constraint inverse filter criterion with a penalty function are investigated in [75]. Penalty functions are designed to extract the various virtual users associated with a given high rate user in "synchronization". The proposed blind detector was compared to a subspace-based blind receiver, a clairvoyant matched fil- 
ter receiver and a known channel/known signatures linear MMSE receiver in terms of the normalized equalization MSE and BER. A new blind and semiblind channel estimation and symbol detection algorithm is developed for long-code W-CDMA systems, including systems with multirate and multicode transmissions in [76]. This technique requires a small number of samples which makes it suitable for fast fading environments. An efficient implementation of the decorrelating RAKE receiver using time-varying state-space techniques is also introduced in this paper.

A successive multistage IC scheme for multirate CDMA systems with either MC, MDM or VPG is implemented in [77], which improves the performance significantly compared to the single-user detector. Both AWGN and Rayleigh fading channels are considered. A multistage PIC structure with hyperbolic tangent decision device designed for asynchronous MC multirate CDMA is analyzed and simulated over AWGN and flat Rayleigh fading channels in [78] assuming both total and partial interference cancellation. Three successive intracell interference cancellation schemes for VPG multirate (downlink) W-CDMA are proposed in [79]. Over the IMT-2000 vehicular channel model (a 6-path multipath channel) the proposed techniques yield a capacity increase compared to conventional single-user detection (with RAKE), while being of low complexity compared to conventional interference cancellation techniques. The performance of MC and MPG multirate methods is evaluated in [80] over AWGN channels by comparing the BER of the decorrrelating, PIC, and GSIC with either PIC or decorrelator with the group. The results show that decorrelating and GSIC yields better performance. Soft feedback interference cancellation scheme is analyzed in [81]. The results show that the performance of SIC depends on the suitable power control and large gain can be achieved under the slow fading channel and high SINR. Multistage SIC and multistage PIC are studied in [5] under perfect, no and imperfect power control and imperfect channel side information. It is found that the performance of 
multistage SIC is always better than that of single or multistage PIC. [82] proposes an energy-add multistage detection method combined with a Cholesky-iterative detection for multirate VPG transmission. It allows symbol-by-symbol detection which is independent of the length of data package and the performance is close to singleuser lower bound. [83] proposes a receiver architecture based on linear PIC scheme for multirate CDMA systems. It does not need the exact knowledge of signature waveforms of the interfering system, instead, it estimates the out-of-rate intersystem interference and subtract by means of its characteristic subspace.

The performance of the hard decision (HD) PIC is studied in [84] under the impact of imperfect channel delay estimation and near-far effect. The 2-stage HD-PIC detector outperforms the matched filter receiver and is near-far resistant when the power difference is small. The results also show that the impact of timing error on a multirate CDMA system is independent of the near-far problem.

A group-wise SIC (GWSIC) receiver structure is proposed in [85] for a dual-rate CDMA system where users are divided into two groups according to the data rates. PIC scheme is employed for the users among the high-rate group with the initial bit estimates determined through MMSE. After the interferences from the high-rate users are regenerated and subtracted or canceled, the bit estimates are obtained for the users among the low-rate group by PIC as well.

[86] proposes an improved hybrid interference cancellation (HIC) receiver for multirate DS-CDMA systems based on VPG. It divides the active users with different data rates into a number of groups, where each group consists of users with different data rates. The group-wise PIC (GWPIC) is performed among the groups, followed by the SIC within each group. The performance of the HIC receiver in AWGN channels is better than that of the adaptive multistage PIC or conventional SIC receiver. 


\subsubsection{Power control in DS-CDMA systems}

Most of the multiuser receivers mentioned earlier aim at providing better detection capabilities by combating MAI and the near-far effect. Power control is another technique to combat the near-far effect that is used in practical DS-CDMA systems employing the conventional single user matched filter (or correlator) detector [87, 88]. Power control consists of regulating the transmitting powers of mobiles users so that the base station receives their signals with approximately the same mean power $[87,89]$. Various power control algorithms have been proposed in the literature (see for example [90]). However, regardless of the method used, power control will not be perfect and imperfect power control yields significant performance degradation $[91,88]$, making the design of near-far resistant receivers important. Looking from another perspective, power control and multiuser detection techniques can be viewed as two different methods to combat near-far effects. Therefore, ways of combining

the two methods have been investigated in the literature. For example, [92, 93, 94] present power control techniques suitable for the SIC scheme. It is shown that as the users are detected successively in the order of decreasing received power or MF outputs, the suitable power control scheme for a SIC detector is to set the power level non-uniformly where the earlier detected users have higher received power so that the SIC can function effectively.

\subsubsection{AME performance}

BER is one commonly used performance measure for comparing multiuser schemes. $\mathrm{AME}$ is another performance analysis tool that is also used to evaluate the performance of wireless CDMA systems. [95] investigates the impact of the imperfect channel estimation on the AME of a two-stage multiuser detector and found that the 
channel mismatch leads to AME degradation over AWGN channels. The AME for an M-stage parallel interference cancellation receiver is presented in [96]. It is found that the M-stage cancellation receiver always outperforms the conventional receiver in terms of AME. As the number of detection stages goes to infinity, the AME over AWGN channels has a value larger than zero for all user energy meaning that the detector is near-far resistant. AME upper and lower bounds are derived in [97] for decision-feedback multiuser detectors. The results show that decision-feedback detection with decorrelated tentative decision achieves better AME over AWGN channels than that with zero-forcing feed-forward equalizer. [98] investigates further the AME for multi-stage decision-directed multiuser detectors and derives a closed-form expression for a two-user system with soft-decision. AME performance of VSL and MC multirate CDMA systems is evaluated for optimum [7, 59], decorrelating and MMSE detectors [59] over AWGN channels showing that VSL outperforms MC in case of equicorrelated correlation matrix but has similar performance for a correlation matrix obtained from random spreading codes. [58] shows that VSL has better AME compared to MC over 2-path Rayleigh channels with optimal detection. Upper and lower bounds of AME are given in [62] for a dual-rate decorrelating decision feedback (DDFB) detector. It shows that DDFB always outperforms the HRD. While DDFB achieves better performance than LRD in the scenario of strong interference, it has worse performance than LRD when the interference is weak. [99] presents an improved MMSE-based receiver and compares its AME performance with the conventional MMSE. In this paper the conventional AME definition was modified by considering the mismatched delay error channel. [100] studies AME for a generalized linear SIC detector for a DS-CDMA system over AWGN channels.

The DBSIC is presented in [101] for a multi-rate CDMA system. Unlike the conventional SIC scheme, at each stage of the DBSIC receiver, a decorrelator on top 
of the conventional MF detector is employed to detect the user's signal to be used at the next stage. Using simulations and analytical analysis, [101] illustrates the performance gains in BER of DBSIC over the conventional single-user MF, decorrelating and conventional SIC detectors under both perfect and imperfect channel side information, but does not consider AME performance.

\subsection{Literature review on channel estimation}

Most single-rate and multirate multiuser detectors require some channel state information (CSI), which needs to be estimated in practice. The amount of CSI required depends on the type of receiver structures considered. If the estimated CSI is not reliable, performance of multiuser detectors may degrade significantly. Therefore, performance of wireless communication systems such as multiuser detectors depends mainly on their ability to obtain an accurate estimation of the CSI they require. Channel estimation consequently is an important issue in mobile communications and accuracy of the channel estimates has a very important impact on the overall system performance. Unlike other types of multiple access schemes, CDMA systems require channel estimation techniques that take into consideration (or "combat" effectively) both noise and MAI.

Channel estimation can be performed either independently of the detection of the unknown data or can be done jointly with the data detection. In other words, either the required CSI is first estimated (using data-aided or blind channel estimation methods such as decision-directed or subspace methods) and then used by the detector, or the receiver structure estimates both the channel parameters and data at the same time (using for example the ML or expectation maximization (EM) algorithm with unknown parameters composed of both data and channel parameters). An intermedi- 
ate approach between independent channel estimation and detection, and "full" joint channel estimation and detection uses one channel estimator block and one detection block that are exchanging parameter information in an iterative way. Channel estimation is said to be data-aided if a known training sequence is used by the receiver for estimating the channel impulse response (CIR). The training sequence or pilot symbols can be transmitted either periodically, let us say every transmission burst, or can be transmitted on a dedicated communication channel (pilot channel). The receiver can utilize the known training bits and the corresponding received samples for estimating CIR, typically for each burst separately in the former case. The training sequence and noise are assumed to be zero mean uncorrelated wide sense stationary processes. Channel estimation methods not requiring training sequences are referred to as blind channel estimation techniques. For example, decision directed approach uses previous data decisions for estimating channel parameters and therefore does not require transmission of a training sequence. Another blind channel estimation technique, referred previously as subspace method exploits the orthogonality between the signal and noise subspaces [102]. Channel estimation techniques requiring an initial training sequence to get an initial channel estimate and then not requiring further training or not as much training could be referred to as semi-blind, or as channel acquisition and tracking methods. Note that decision-directed approach is often used in a tracking mode, e.g. after initial CSI estimates have been obtained using a dataaided or blind technique. Generally, channel estimation techniques can be performed in the time and/or frequency domain and can be classified as either single-user or multiuser.

ML approach is an optimum method for both joint data detection and channel estimation, or channel estimation alone based on the data-aided or decision-directed approaches. The joint ML estimator (or the ML estimator respectively) determines 
the channel vector and data (or the channel vector respectively) that maximize the conditional likelihood function conditioned on the unknown parameters [103].

Let us focus on ML channel estimation based on the data-aided or decision-directed approaches. In that case, a known training sequence has been sent or the transmitted sequence has been obtained using a decision-directed approach. What remains unknown are the channel parameters. Two cases can be considered, either the channel vector is assumed to be deterministic and unknown, and an estimate of the unknown channel vector is to be found, or the channel vector is assumed to be a random vector whose particular realization needs to be estimated. In both cases, a general likelihood expression needs to be maximized leading into a nonlinear multidimensional search over the parameters space. One way around the computational complexity problem is to apply the EM method [104]. The EM algorithm enables to find iteratively the ML estimate by decomposing large multi-dimensional ML problems into smaller ones.

If the channel information and its statistics are completely unknown, the ML estimator is the best estimator that can be obtained. Generally, we have some knowledge about the degree of correlation of the channel gains in time. One way to exploit this knowledge is to use smoothing filter to improve estimation results. An alternative approach is to consider the channel information to be a realization of a random process to be estimated by the channel estimation algorithm, which leads to the use of a Kalman filter. The Kalman filter is a time-varying linear filter widely used in statistical estimation when the unknown parameters evolve in time according to some dynamic model. In particular, for Rayleigh fading channels, the time-variant channel coefficients can be modeled as a zero-mean complex Gaussian process with autocorrelation function in the form of the zero ${ }^{\text {th }}$-order Bessel function of the first kind (which is a function of the Doppler frequency). If the Doppler frequency were known here, the channel could be modeled by an auto regressive (AR) process, and the optimal 
channel estimator would be a Kalman filter.

$[105,106]$ considers ML estimation of multipath channel parameters in asynchronous DS-CDMA communication systems assuming that the user whose channel parameters are to be estimated transmits a training sequence. By modeling the interfering signals as unknown colored Gaussian noise, the multiuser estimation problem is decomposed into a series of single user problems. [107] develops a ML channel estimation method for synchronous CDMA systems, which uses the known spreading sequences together with short training sequences. [108] proposes a joint ML multiuser channel estimation method for CDMA systems over multipath fading channels. The joint channel estimator uses the RAKE outputs at the symbol rate and assumes perfect knowledge of the signature and energies of all users, as well as their transmitted symbols (obtained either by using pilots symbols (training sequences) or by prior detection). [109] derives the ML channel estimate for asynchronous long-code CDMA systems over multipath channels using training sequences and approximates it using an iterative algorithm to reduce the computational complexity per bit. The proposed iterative channel estimation technique is extended to track slowly varying multipath fading channels using decision feedback (decision-directed method) assuming multistage PIC detection where bits are detected in blocks. The obtained multiuser channel estimation and tracking scheme is shown to be MAI-resistant. [110] investigates the problem of channel tracking for RAKE receivers in closely spaced multipath environments. The system model considers CDMA transmission where the interference from other users is modeled as additional noise. Both timing tracking and phasor estimator are considered. The principle of the phasor estimator is as follows: ML estimates from each Rake finger are generated and postprocessed by an interference canceller and then passed through a bank of Wiener filters (one for each RAKE finger). This approach partly cancels multipath interference. [111] addresses the problem of chan- 
nel estimation for multiple data rate DS-CDMA systems and proposes data-aided downlink and uplinks algorithms based on approximate solutions to ML estimation, that are suitable for both VC and VSL multirate systems. Direct exploitation of the multirate nature of the signal yields improved performance compared to single-rate estimation.

In [112] it is shown that when spatial/ temporal diversity is available at the receiver, a subspace technique can be directly applied to downlink channel estimation of long code CDMA systems. Detection of the desired user can be performed by typical linear techniques after channel is estimated. The method depends on eigenvalue decomposition on the data covariance matrix which is typically estimated from finite data samples. Using a unified model, [113] proposes a subspace method for channel estimation applicable to VPG, MC and VC multirate CDMA systems. A reduced complexity method is also proposed and performance/complexity trade-offs are investigated. In a similar work but including adaptation [114] proposes a subspacebased blind adaptive channel estimation algorithm for dual-rate quasi-synchronous DS-CDMA systems and shows that the proposed algorithm has better performance at the low-rate mode than at the high-rate mode, but at the expense of an increasing computational complexity for the low rate mode.

[115] investigates the transmission over time-variant multipath Rayleigh fading channels employing DS-CDMA. An iterated channel estimation scheme employing pilot symbols in conjunction with soft decision interference cancellation is applied to improve the reliability of the channel estimation as well as data symbol detection considerably. [116] considers a blind multiuser detection in uplink asynchronous CDMA systems employing aperiodic (long) spreading sequences based on the sequential EM algorithm. The receiver sequentially estimates the channel using the sequential EM algorithm and the snapshot estimates of each path are tracked by linear MMSE filters. 
The user data are detected by a ML sequence detector with the channel estimates information. [117] studied the problem of joint blind channel estimation and MAI suppression for an asynchronous CDMA system. A low-complexity sliding-window scheme based on the EM algorithm is developed for joint blind maximum a-posteriori probability (MAP) multiuser detection and stochastic ML channel estimation in a dispersive fading channel. The proposed scheme can track fading channels with no phase ambiguities even when channel gains are close to zero. [118] uses a ML approach to estimate an effective spreading code vector for each user which captures all channel parameters. The channel estimation algorithm is designed to estimate the channel vector in a computational efficient manner, instead of focusing on estimation of the individual channel parameters. The estimated effective spreading code is applied in the detection process and a computationally efficient solution is developed for the combined problem of channel estimation and multi-shot detection in a scenario involving multiple users, multiple paths, and multiple sensors.

Channel estimates can also be obtained using linear MMSE (LMMSE) method. Let $\vec{y}=\mathbf{R} \mathbf{b} \vec{\alpha}+\vec{n}$. The LMMSE algorithm for channel estimation computes the optimum matrix $\mathbf{W}$ such that the mean square error $E\left[\left\|\vec{\alpha}-\mathbf{W}^{*} \vec{y}\right\|^{2}\right]$ is minimized [119]. Then the linear MMSE estimate of $\vec{\alpha}$ can be obtained as $\hat{\vec{\alpha}}=\mathbf{W}^{*} \vec{y}$. [120] addresses LMMSE channel estimation based on a pilot transmission and the impact of channel estimation errors on coherent receiver performance. It derives a simple characterization of the MMSE estimator in terms of a bank of filters and shows that performance of the estimator depends on the effective SNR, product of pilot SNR and normalized coherence time. [121] considers the problem of pilot-symbol-aided estimation of multipath fading channels in uplink CDMA systems. It divides the transmitted symbol streams of each user into time-slots where each time-slot contains a number of pilot-symbols followed by information data symbols. Channel estimation is based 
on interpolation of the channel values corresponding to the pilot symbols in adjacent time-slots. Two new channel estimation methods (the robust channel interpolator, and the polynomial channel interpolator) are developed. [122] compares the BER performance of differential detection and pilot symbol-assisted coherent detection on the frequency-selective Rayleigh fading channel assuming an equal gain or a maximal ratio combining RAKE receiver. The results show the performance advantage of rate 1/8 convolutionally coded pilot symbol-assisted BPSK over coded differential detection. [123] proposes several algorithms including a subspace approach (based on a subspace decomposition of the data), a pilot-aided approach (based on exploiting a pilot channel), and a decision-based approach (based on a decision-based technique) for channel estimation of a synchronous CDMA point-to-multipoint link (downlink) that uses aperiodic spreading waveforms. The decision-based approach achieves the Cramer-Rao bound for realistic SNR and integration times. [124] proposes a pilotassisted MMSE multiuser channel estimation algorithm for quasi-synchronous CDMA systems that undergo frequency-selective channel fading. The multiuser channel tap coefficients at pilot symbol positions are estimated under the MMSE criterion with the help of the channel intertap correlation matrix, which is determined by the combined effects of the physical fading channel, transmit filter, and receive filter. A novel iterative method is proposed for the joint estimation of the channel intertap correlation and filter tap timing based on the received pilot samples. [125] addressed single-user channel estimation in multiuser DS-CDMA systems for the case of sparse channels with large delay spreads. In particular, it is proposed to use different spreading codes for the estimation of the fractional and integer part of the delays to achieve optimized estimation of these quantities.

Adaptive channel estimators can be implemented using the LMS algorithm which depends on a parameter $\mu$ called the step size. LMS algorithm is still widely used due 
to its simplicity and cheap implementation. It is seen as having low computational complexity, good stability properties, relatively good robustness against implementation errors and simplicity of its behavior. The disadvantages of LMS include poor performance due to oversimplified channel model which does not take into account correlations of the channel. The LMS adaptation rule is quite sensitive to the step size parameter. When selecting this parameter value, a wrong selection may ruin a long simulation due to possible divergence problems. It is better to be sure that the selected parameter value is suitable for the whole SNR range of interest and all possible channel profiles. Variable step size $\mu$ can be used in LMS algorithm through gradient descent method to adjust the $\mu$ parameter during the adaptation process where the adjustment depends on the channel estimation error. Another recursive least squares (RSL) scheme, which is more complicated than LMS, can offer faster convergence solution. RSL also implements a variable step size which is recursively defined but the selection of step size does not depend on the channel estimation error [126]. [127] proposed a joint multiuser detection and channel estimation scheme that includes a path-by-path decorrelator, a channel estimator based on the RSL algorithm and a channel-matched decorrelator. The path-by-path decorrelator is used for providing the channel estimator with the noisy channel information path-by-path (using training sequences). Decisions are made on the output of the channel-matched decorrelator and are fed back to the channel estimator to be used as the reference signals. The received complex envelope of each of the propagation paths is then estimated in the channel estimator using RSL algorithm.

[128] analyzes the BER performance of general linear coherent multiuser receivers with diversity reception and imperfect channel estimation using data-aided channel or decision-directed estimation. For data-aided channel estimation, both exact BERs and low-complexity BER approximations using the multivariate Gaussian approximation 
(MGA) are presented. A BER approximation for receivers with decision-directed channel estimation using a Markov chain steady-state analysis is also proposed. 


\section{Chapter 3}

\section{Multiuser Multirate Asynchronous}

\section{DS-CDMA Receivers}

Fig. 3.1 displays the block diagram for a single-rate DS-CDMA system. The data from each physical user are spread and scrambled by the signature/spreading codes, and transmitted through the AWGN and/or multipath fading channel. During transmission signals are corrupted by MAI, AWGN and faded by multipath effect. The corrupted signals that arrive at the receiver are processed through the matched filter (for AWGN channels), or the RAKE receiver (for multipath fading channels), the despreader and the multiuser detector to recover the transmitted signals. This classical DS-CDMA block diagram will be slightly modified in the case of multirate DS-CDMA systems as will be seen next.

\subsection{Transmitted signals for a multirate VPG CDMA system}

An asynchronous multi-rate DS-CDMA system is considered which consists of $C$ data rate classes and $K_{c}$ physical users in class c for a total of $K=\sum_{c=1}^{C} K_{c}$ users. VPG is 


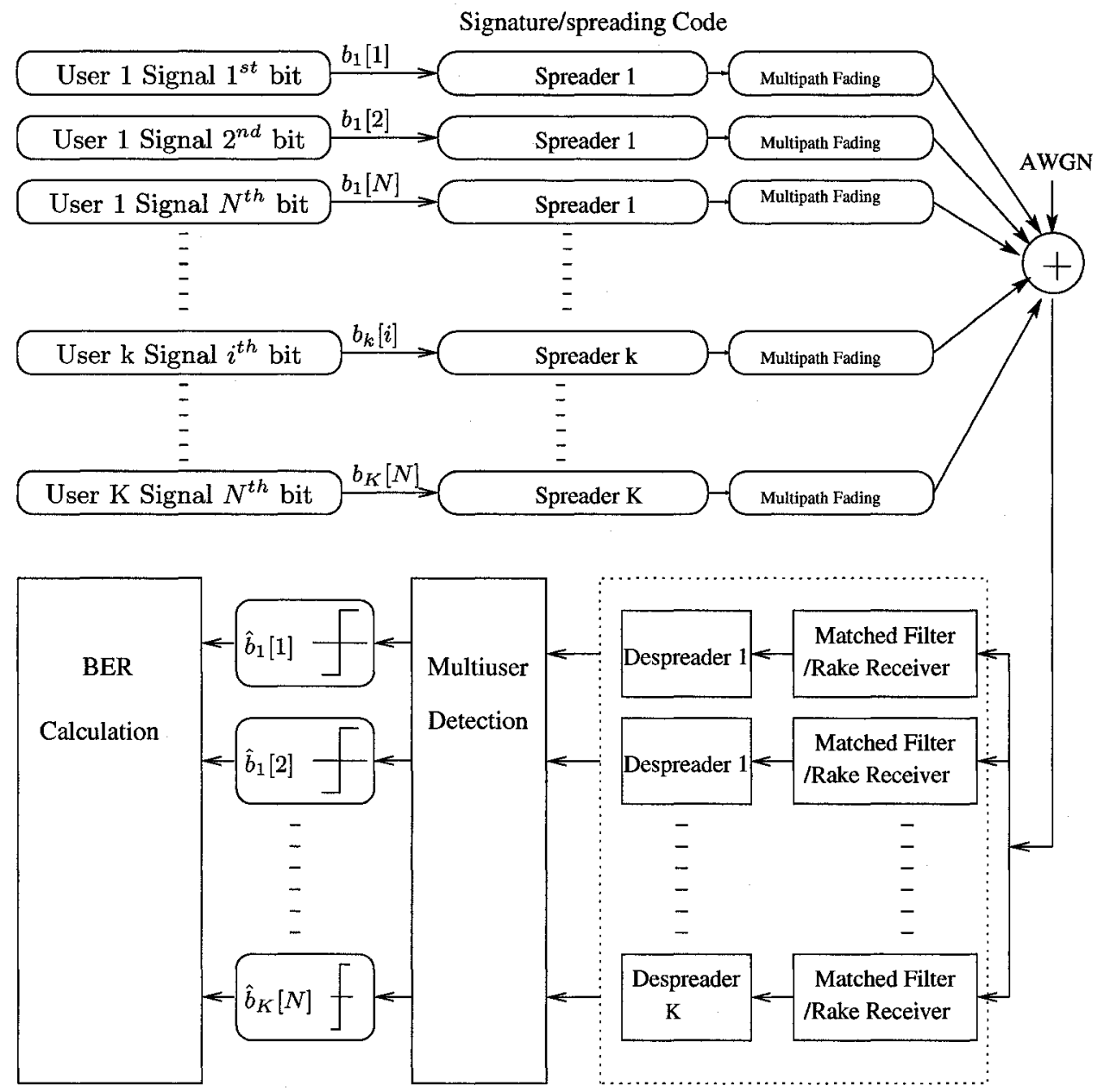

Fig. 3.1 Block diagram for a single-rate DS-CDMA system

employed in this multi-rate communication system where physical users with lowest data rate have the largest processing gain and physical users with highest data rate have the smallest processing gain. The data rate for class $c$ is denoted as $R_{c}$. The slowest data rate or base rate is denoted as $R$ which is the same as the data rate of class $1, R_{1}$. Assuming that $R_{c}=c R$, where $c$ is an integer, each physical user in class $c$ with data rate $R_{c}$ can be treated as $c$ independent virtual users with base data rate $R$. Indeed, during $T_{s}$ the duration of one symbol of the slowest rate users, a physical user of class $c$ transmits $c$ symbols since $R_{c}=c R$. The total number of virtual users 
is therefore $K_{v}=\sum_{c=1}^{C} c K_{c}$. Fig. 3.2 shows the virtual users' signals for the simulated multirate CDMA system.

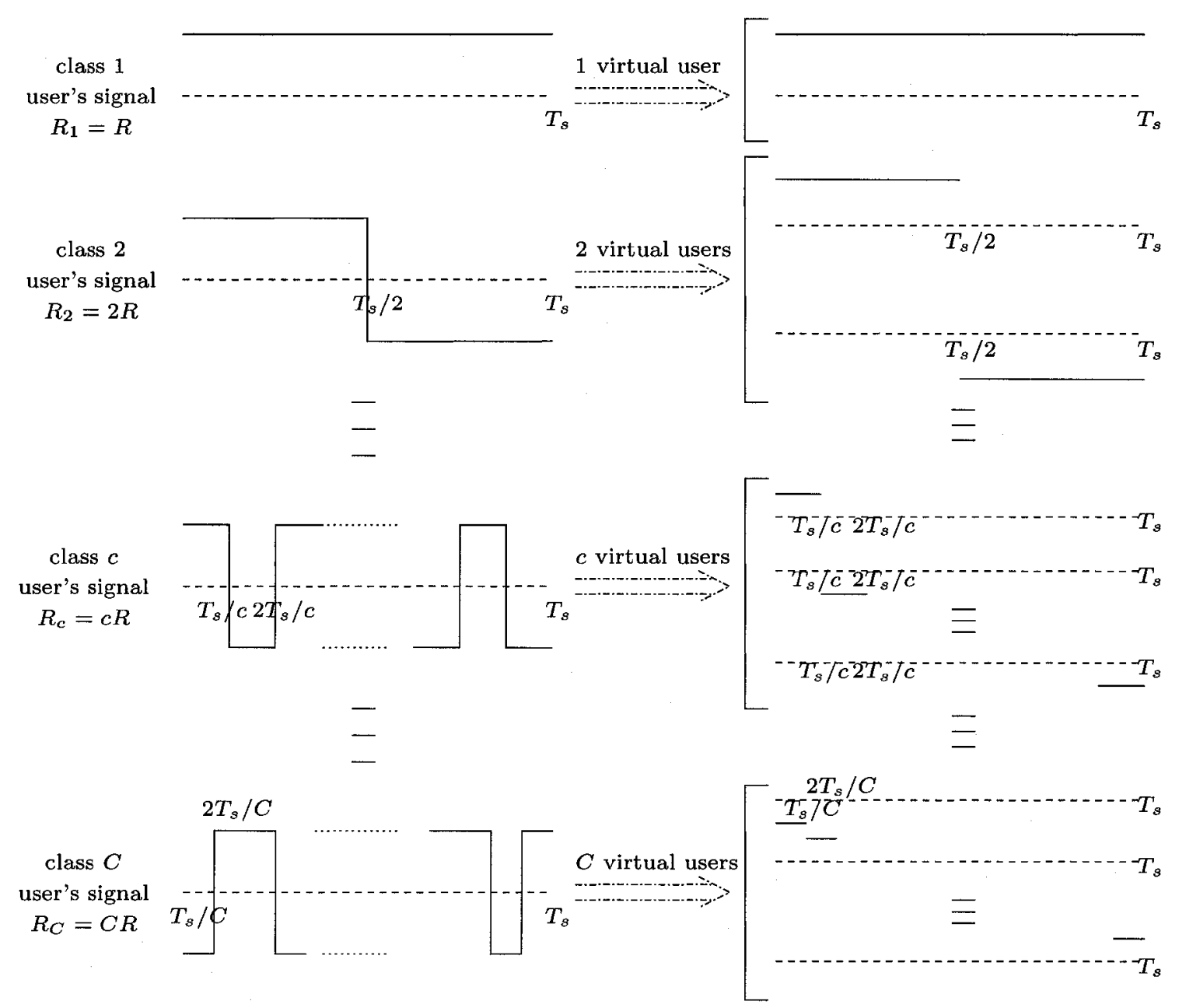

Fig. 3.2 Virtual users' signals for the simulated multirate CDMA system

Spreading sequences For both single rate or multirate DS-CDMA systems, users signals have to be spread using spreading codes. Spreading codes cannot be chosen arbitrarily if one wants to ensure good performance of a DS-CDMA system. Requirements for the spreading codes include good auto-correlation properties (for separating different paths) and good cross-correlation properties (for separating different chan- 
nels). The autocorrelation functions should be impulse like and self-orthogonal codes are desirable. Cross correlation should be as low as possible for suppression of other user signals. Orthogonal codes are the best. However, fulfilling simultaneously good auto and cross correlation properties is impossible and trade-off is required. A spreading code has the property of periodic repetition where each data symbol is spread with the same code. Depending on the period of the spreading code, the spreading code can be classified as long code or short code. For long codes the period of the spreading code is much longer than the data symbol duration. They are often used for additional scrambling for suppression of asynchronous signal parts. Short codes are used for channel separation in uplink and downlink. The spreading codes can also be classified as orthogonal or non-orthogonal codes.

Orthogonal Spreading Code: Walsh sequence (code) is an orthogonal spreading code and is obtained from Hadamard Codes by BPSK modulation (through recursive construction with matrices). They can achieve perfect suppression of other signals for synchronous transmission but very poor performance for asynchronous transmission due to the bad correlation properties in that case. Hence, they are only used in combination with scrambling codes in practice.

Maximum Length Sequences ( $m$-sequences): One of the non-orthogonal spreading codes is $\mathrm{m}$-sequence which is generated by linear feedback shift register (LFSR) as a Pseudo-Random (PN) sequence. A PN sequence is a binary sequence of $1 \mathrm{~s}$ or $0 \mathrm{~s}$ with an autocorrelation that is approximately equal to that of a random binary sequence. Strictly speaking a PN sequence is not random at all instead it is a deterministic periodic signal. However it has statistical characteristics that are similar to those of random sequences such as white noise. One important feature of PN sequences in 
CDMA systems is that there is a very low correlation among the shifted versions of the sequences and PN sequences ensure good MAI rejection capability. The output of an LFSR sequence generator depends on the number of stages, the feedback tap connections, and the initial conditions. A feedback configuration can be found to give a period of $2^{n}-1$ ( $n$ is the number of the shift registers) and leads to a m-sequence which has $2^{n-1}$ ones and $2^{n-1}-1$ zeros. M-sequences are periodical sequences and have very good autocorrelation properties, but poor cross correlation. There exist only few sequences for given lengths of $n$. Primitive polynomial for feedback ensures maximum period of $2^{n}-1$.

Gold Sequences: Gold sequences are one kind of PN sequences and are used to scramble and spread data in CDMA systems for the purpose of separating the signals from different users and/or reducing the interference produced by other users. Spreading sequences with good statistical properties can be generated from PN Gold sequences. Each user has a distinct Gold code and the code is known both at the transmitter and receiver [129]. Since Gold sequences yield odd processing gains, to obtain even processing gains, one random chip is added to the Gold sequences [80].

A Gold sequence $G_{c, k}(t)$ of length $G_{c}$ can be written as:

$$
G_{c, k}(t)=\sum_{i=1}^{G_{c}}\left\{a_{c, k}[i] \frac{1}{\sqrt{G_{c}}} P\left(t-(i-1) T_{c h}\right)\right\}
$$

where $P(t)$ is a pulse of duration $T_{c h}$ and $a_{c, k}[i]$ is the value of $i^{t h}$ chip for user $k$ of class $c$. In this thesis, $P(t)$ is assumed to be a rectangular pulse with unit energy, and no filtering is assumed in the user transmitted signals.

Gold codes consist of combination of preferred pair of m-sequences and are generated by the XOR of a pair of preferred m-sequences, with the output being the same 
length as the two maximal codes, but it is nonmaximal. Over a set of codes available from a given generator, the cross-correlation between the two codes is uniform and bounded. The procedures to generate Gold sequence can be as follows: start with two preferred m-sequences, $m_{1}$ and $m_{2} ;$ XOR these two sequences and a new sequence $m_{3}$ can be produced; shift $m_{2}$ by one and XOR this sequence with $m_{1}$ to produce $m_{4}$; repeat the procedures to get $m_{5}, \cdots ;$ the sequences $m_{1}, m_{2}, m_{3}, \cdots$ are Gold sequences.

Table 3.1 lists the preferred pairs (polynomial 1 and 2) for the generation of Gold sequences.

\begin{tabular}{|r|r|r|r|}
\hline$n$ & $G$ & Preferred polynomial 1 & Preferred polynomial 2 \\
\hline 4 & 15 & $10011_{2}=19_{10}$ & $111101_{2}=61_{10}$ \\
5 & 31 & $100101_{2}=37_{10}$ & $11111_{2}=31_{10}$ \\
6 & 63 & $1000011_{2}=67_{10}$ & $1100111_{2}=103_{10}$ \\
7 & 127 & $10001001_{2}=137_{10}$ & $10001111_{2}=143_{10}$ \\
\hline
\end{tabular}

Table 3.1 Preferred Pairs for Gold Sequences

The cross-correlation between any two sequences takes on one of the values $-t(n),-1$, $t(n)-2$, where:

$$
t(n)= \begin{cases}2^{(n+1) / 2}+1 & \text { odd } \mathrm{n} \\ 2^{(n+2) / 2}+1 & \text { even } \mathrm{n} .\end{cases}
$$

There are two ways of choosing the spreading sequences of the virtual users corresponding to one high rate physical user. Equivalently, this corresponds to two ways of implementing a VPG system. 
Signature sequences of virtual users (first method) In the first method, the $k^{\text {th }}$ physical user of class $c$ is assigned a spreading sequence of length $G_{c}$

$$
S_{c, k}(t)=\sum_{i=1}^{G_{c}}\left\{a_{c, k}[i] \frac{1}{\sqrt{G_{c}}} P\left(t-(i-1) T_{c h}\right)\right\}
$$

where $G_{c}$ is the processing gain of all users of class $c, a_{c, k}[i](\in\{-1,1\})$ is the value of the $i^{\text {th }}$ chip for user $k$ of class $c, T_{c h}$ is the chip duration, $P(t)$ is the chip pulse. The virtual users derived from a real physical user have the same signature codes but occupying different time slots in one symbol interval. Mathematically, the signature sequence of the $j^{\text {th }}$ virtual user corresponding to user $k$ of class $c S_{c, k}^{(j)}(t)$ is defined as follows:

$$
\left\{S_{1, k}^{(1)}(t)=S_{1, k}(t) \quad\left\{\begin{array} { c } 
{ S _ { 2 , k } ^ { ( 1 ) } ( t ) = S _ { 2 , k } ( t ) } \\
{ S _ { 2 , k } ^ { ( 2 ) } ( t ) = S _ { 2 , k } ( t - T _ { 2 , s } ) }
\end{array} \cdots \left\{\begin{array}{c}
S_{C, k}^{(1)}(t)=S_{C, k}(t) \\
\vdots \\
S_{C, k}^{(j)}(t)=S_{C, k}\left(t-(j-1) T_{C, s}\right) \\
\vdots \\
S_{C, k}^{(C)}(t)=S_{C, k}\left(t-(C-1) T_{C, s}\right)
\end{array}\right.\right.\right.
$$

Equivalently

$$
S_{c, k}^{(j)}(t)=S_{c, k}\left(t-(j-1) T_{c, s}\right) \quad 0 \leq t \leq T_{s} \quad \begin{aligned}
& j=1, \ldots, c \\
& c=1, \ldots, C
\end{aligned} \quad k=1, \ldots, K_{c}
$$

where $T_{c, s}=1 / R_{c}=T_{1, s} / c=T_{s} / c$ is the symbol interval for class $c$ users. 
Signature sequence of virtual users (second method) In the second method, the $k^{\text {th }}$ physical user of class $c$ is assigned a spreading sequence of length $G_{1}$

$$
S_{c, k}(t)=\sum_{i=1}^{G_{1}}\left\{a_{c, k}[i] \frac{1}{\sqrt{G_{1}}} P\left(t-(i-1) T_{c h}\right)\right\}
$$

where $G_{1}$ is the processing gain for the slowest rate users (i.e. the highest processing gain). In other words, regardless of their rates all the physical users are assigned spreading sequences of equal lengths. The signature codes of the virtual users derived from a real physical user are obtained by considering only a portion of the spreading sequence of length $G_{1}$. Mathematically, the signature sequence of the $j^{\text {th }}$ virtual user corresponding to user $k$ of class $c S_{c, k}^{(j)}(t)$ is defined as follows: for $0 \leq t \leq T_{s}$ we have

$$
\begin{aligned}
& \left\{S_{1, k}^{(\mathbf{1})}(t)=S_{1, k}(t) \quad 0<t<T_{1, s} \quad\left(\text { recall } T_{1, s}=T_{s}\right)\right.
\end{aligned}
$$

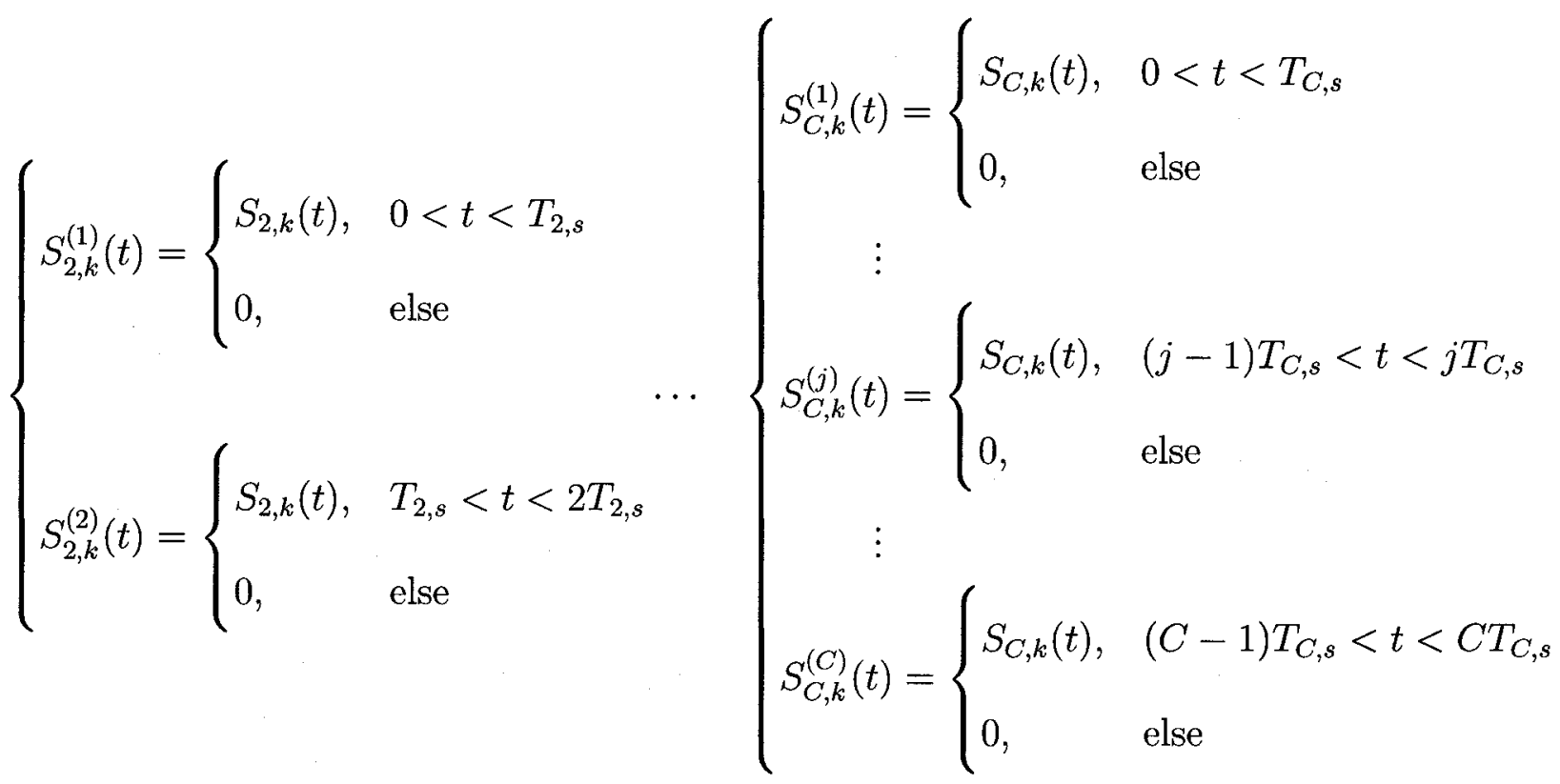


Equivalently for $0 \leq t \leq T_{s}$

$$
S_{c, k}^{(j)}(t)=\left\{\begin{array}{lll}
S_{c, k}(t), & (j-1) T_{c, s}<t<j T_{c, s} & j=1, \ldots, c \\
0, & \text { else } & c=1, \ldots, C
\end{array} \quad k=1, \ldots, K_{c}\right.
$$

For example let us consider a dual-rate asynchronous DS-CDMA system containing three low-rate users and two high-rate users where the processing gain of the low-rate users is 32 and the processing gain of the high-rate users is 16. An initial random signature sequence of length 32 is assigned to each physical user. Each physical high rate user can be treated as two virtual users. The spreading sequence for the first virtual user can be formed by adding 16 ' 0 's after the first half of the 32 chips to make the total length of 32 . The spreading sequence for the second virtual user is adding 16 ' 0 's before the second half of the same 32 chips. A full 32-bit spreading sequence is assigned to each one of the low rate user. The total number of the virtual users is considered as seven and the corresponding 32-bit signature sequences are listed in Table 3.2, where the number in bracket in the virtual users column corresponds to the index $j$. Since we are considering even processing, the spreading sequences $S_{c, k}(t)$ are generated by adding one random chip to Gold sequences [80].

Multirate multiuser transmitted signal Assuming antipodal BPSK modulation, the transmitted signal of the $j^{\text {th }}$ virtual user derived from user $k$ of class $c$ corresponding to an information sequence of length $N$ (with respect to class 1 users) can be written in the baseband format as:

$$
x_{c, k}^{(j)}(t)=\sum_{i=1}^{N}\left\{\sqrt{E_{c, k}^{(j)}} b_{c, k}^{(j)}[i] S_{c, k}^{(j)}\left(t-\tau_{c, k}^{(j)}-(i-1) T_{s}\right)\right\}
$$




\begin{tabular}{|c|c|c|c|c|}
\hline \multicolumn{2}{|c|}{$\begin{array}{l}\text { physical } \\
\text { users }\end{array}$} & \multirow{2}{*}{$\begin{array}{c}\text { 32-bit sequence } \\
\text { physical users } \\
S_{c, k}\end{array}$} & \multirow{2}{*}{$\begin{array}{c}\text { virtual } \\
\text { users } \\
\text { No. }(j) \\
\end{array}$} & \multirow{2}{*}{$\begin{array}{c}\text { 32-bit sequence } \\
\text { virtual users } \\
S_{c, k}^{(j)} \\
\end{array}$} \\
\hline class & No. & & & \\
\hline 1 & 1 & $S_{1,1}$ & $1(1)$ & $S_{1,1}$ \\
\hline 1 & 2 & $S_{1,2}$ & $2(1)$ & $S_{1,2}$ \\
\hline 1 & 3 & $S_{1,3}$ & $3(1)$ & $S_{1,3}$ \\
\hline \multirow[t]{2}{*}{2} & \multirow[t]{2}{*}{1} & \multirow[t]{2}{*}{$S_{2,1}=\left(S_{2,1_{\text {first-half }}}, S_{2,1_{\text {second-half }}}\right)$} & $4 \quad(1)$ & $\left(S_{2,1_{\text {first-half }}}\right),(0, \cdots, 0)_{16}$ \\
\hline & & & $5(2)$ & $(0, \cdots, 0)_{16},\left(S_{2,1_{\text {second-half }}}\right)$ \\
\hline \multirow[t]{2}{*}{2} & \multirow[t]{2}{*}{2} & \multirow{2}{*}{$S_{2,2}=\left(S_{2,2_{\text {first-half }}}, S_{2,2_{\text {second-half }}}\right)$} & $6(1)$ & $\left(S_{2,2_{\text {first-half }}}\right),(0, \cdots, 0)_{16}$ \\
\hline & & & $7(2)$ & $(0, \cdots, 0)_{16},\left(S_{2,2_{\text {second-half }}}\right)$ \\
\hline
\end{tabular}

Table 3.2 Signature Sequences for a 3-low-rate and 2-high-rate system

where $b_{c, k}^{(j)}[i]=i^{\text {th }}$ bit of $j^{\text {th }}$ virtual user derived from user $k$ of class $c$ and $\in\{-1,1\}$; $E_{c, k}^{(j)} / c$ is the transmitted signal energy of the $j^{\text {th }}$ virtual user derived from user $k$ in class $c, S_{c, k}^{(j)}(t)=$ signature waveform of $j^{t h}$ virtual user derived from user $k$ of class c. $T_{s}=T_{1, s}$ is the symbol duration for the slowest class users, $\tau_{c, k}^{(j)}$ is the delay of the $j^{\text {th }}$ virtual user derived from user $k$ of class $c$ (is independent of $j$ and is equal to the delay of user $k$ of class $c$ ). All virtual users associated with the same physical user have the same delay. The delay of each physical user is assumed to be an integer of $T_{c h}$ (chip interval) and less than $T_{s}$, and is uniformly randomly generated. In other words, the system is assumed to be symbol-asynchronous but chip-synchronous.

\subsection{Channel model and received signal}

Radio waves propagate from a transmitting antenna through mobile free space channel and are affected by shadowing (large scale fading) and multipath fading (small-scale fading) [130].

Large-scale fading is primarily attributed to path loss when the received signal strength decays over relatively large distances (several hundreds or thousands of me- 
ters) between the transmitter and the receiver. It is also known as slow fading or shadowing. Shadowing is a long-term gross variation in the mean signal amplitude level and caused by the movement over distances large enough between the transmitter and the receiver to cause gross variations in the overall path.

The rapid fluctuations in the received signal amplitude over a short period of time or travel distance is called small-scale fading, and is observed over distances of about half a wavelength. These short-term fluctuations in the received signal amplitude are caused by atmospheric reflection, refraction, diffraction, and scattering from ground terrain, the atmosphere such as building, bridges, hills, trees and other objects in the path.

Reflection occurs when a propagating electromagnetic wave encounters a surface that is large relative to the wavelength of the propagating wave. This reflected wave may interfere constructively or destructively at the receiver due to the change in phase shift after reflection, resulting in the fading phenomenon. Sources for reflections include the surface of the earth, buildings and walls.

Diffraction occurs at the edge of an impenetrable body or at a surface with sharp irregularities that is large compared to the wavelength of the radio wave. The secondary waves resulting from such edges or surfaces are partially reflected and retransmitted with a bend of waves around the obstacle, also creating fading. This allows the signal to be transmitted even when there is no line-of-sight (LOS) path between the transmitter and the receiver.

Scattering occurs when the radio path between the transmitter and receiver consists of large amount of objects with dimensions that are small compared to the wavelength of the signal. The scattered waves can be produced by rough surfaces or by other irregularities in the channel such as foliage and traffic signs.

In most mobile or cellular systems, the height of the mobile antenna may be smaller 
than the surrounding structures. Thus, the existence of a direct or LOS path between the transmitter and the receiver is highly unlikely. In such a case, propagation is mainly due to reflection and scattering from the buildings and by diffraction over and/or around them. So, in practice, the transmitted signal arrives at the receiver via several paths with different time delays creating a multipath situation.

These multiple physical phenomena are responsible for most of the characteristic features of the received signal. The random phase and amplitudes of the different multipath components cause fluctuations in signal strength therefore inducing multipath fading.

Multipath fading can be classified as flat (frequency non-selective) or frequency selective based on multipath time delay spread, and slow or fast based on Doppler spread. These two types of fading are independent of each other.

Flat fading occurs when the transmitted signal has a narrow bandwidth which is less than the coherence bandwidth of the channel (the baseband signal has a duration greater than the excess delay of the channel). Equivalently, flat fading occurs when the mobile radio channel has a constant gain and a linear phase response over a bandwidth larger than the bandwidth of the transmitted signal. Under these conditions, the strength of the received signal might change with time (the received signal has amplitude fluctuations due to the variations in the channel gain over time caused by multipath). However, all the spectral components go through the same effect where the spectral characteristics of the transmitted signal are maintained at the receiver. Flat-fading can cause a dramatic increase in either the average bit-error-rate or the signal outage probability.

Frequency selective fading occurs when the bandwidth of the transmitted signal is larger than the coherence bandwidth of channel (the delay spread is larger than the symbol period). The mobile channel has a constant gain and a linear phase 
response over a bandwidth smaller than that of the transmitted signal. In that case, the received signal includes multiple versions of the transmitted waveform, attenuated and delayed in time. So the received signal is distorted and dispersed. The channel is said to be frequency selective as it has different gains for different frequencies. This leads to time dispersion of the transmitted symbols within the channel arising from these different time delays resulting in ISI, which causes an irreducible error floor in performance if ISI is not mitigated.

Wireless channels also exhibit Doppler shift which causes spectral broadening and leads to adjacent channel interference. The objects in the radio channel are generally in motion and relative motion of the mobile with respect to the transmitter antenna causes different Doppler frequency shifts in the received signal spectrum, increasing the signal bandwidth. The channel impulse response changes rapidly within the symbol duration. This causes frequency dispersion due to Doppler spreading, which leads to signal distortion. Fast fading occurs when the Doppler spread is significant larger than the bandwidth of the transmitted baseband signal (the coherence time is smaller than the symbol period). This form of fading typically occurs for very low data rates. Slow fading occurs when the Doppler spread of the channel is much less than the bandwidth of the baseband signal (the coherence time is larger than the symbol period).

This thesis focuses mainly on the three propagation mechanisms that cause the occurrence of small-scale multipath fading. The multipath signals are time-shifted signals and can be represented as taps in an impulse-response model of a channel. The well known Gaussian wide sense stationary uncorrelated scattering (GWSSUS) model is applied for the stochastic properties of the time varying path/tap weights. Based on the GWSSUS model, throughout this work the particular case of zero mean path weights whose magnitudes are Rayleigh distributed is considered. For a GWSSUS 
channel, the uncorrelated multipaths are commonly assumed to be chip spaced for DS-CDMA systems, but is generally not common in practice due to the random nature of multipaths. This assumption leads the fading channels to be represented as tapped delay line (TDL) filters whose taps are statistically uncorrelated.

Transmission over a slow Rayleigh multipath fading channel is considered (Fig. 3.3). The fading is assumed to be sufficiently slow such that the fading is constant over a sequence of $N$ symbols. Modeling the multipath Rayleigh fading channel as a truncated tapped delay line with taps delays equal to the chip duration $T_{c h}$, the complex low-pass equivalent impulse response of the channel is expressed as

$$
h(t)=\sum_{l=1}^{L} \alpha[l] \delta\left(t-(l-1) T_{c h}\right) \quad 0 \leq t \leq N T_{s}
$$

where $L$ is the number of paths, $T_{c h}$, equal to the chip duration, is the delay of the tapped-delay line, for all $l . \alpha[l]$ are uncorrelated zero-mean complex valued Gaussian random variables (i.e. the envelope $|\alpha[l]|$ is Rayleigh-distributed).

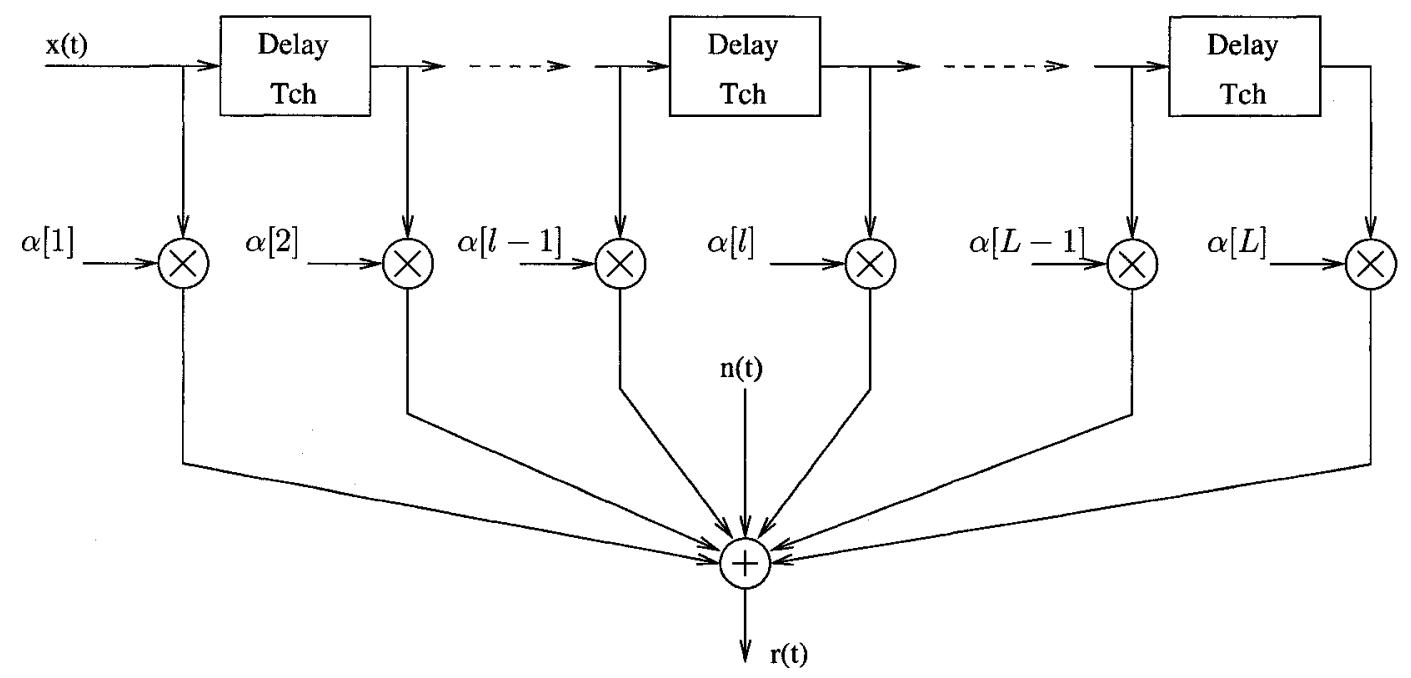

Fig. 3.3 Multipath Fading Channel Model 
The received signal is the convolution of the transmitted signals and the channel impulse response plus the additive noise and is given by:

$r(t)=\sum_{i=1}^{N} \sum_{c=1}^{C} \sum_{k=1}^{K_{c}} \sum_{j=1}^{c} \sum_{l=1}^{L}\left\{\sqrt{E_{c, k}^{(j)}} b_{c, k}^{(j)}[i] \alpha_{c, k}^{(j)}[l] S_{c, k}^{(j)}\left(t-\tau_{c, k}^{(j)}-(l-1) T_{c h}-(i-1) T_{s}\right)\right\}+n(t)$

where $\alpha_{c, k}^{(j)}[l]$ is the $l^{t h}$ path gain of the $j^{\text {th }}$ virtual user derived from the $k^{\text {th }}$ physical user of class $c$ (modeled as a zero mean circularly complex Gaussian random variable). The noise $n(t)$ is a complex Gaussian random process with zero mean and power spectral density $\frac{N_{0}}{2}$.

For simplicity of notation, the virtual users for all physical users for all classes are ordered such that the $j^{\text {th }}$ virtual user derived from the $k^{\text {th }}$ user of class $c$ is assigned the index $m \in\left\{1, \ldots, K_{v}\right\}$ defined as $m=\sum_{i=0}^{c-1} i K_{i}+c(k-1)+j$, where $j \in\{1, \ldots, c\}$, $k \in\left\{1, \ldots, K_{c}\right\}, c \in\{1, \ldots C\}$, and $K_{v}=\sum_{c=1}^{C} c K_{c}$ is the total number of virtual users. Therefore the received signal (3.8) can be re-written as:

$$
r(t)=\sum_{i=1}^{N} \sum_{m=1}^{K_{v}} \sum_{l=1}^{L} \sqrt{E_{m}} b_{m}[i] \alpha_{m}[l] S_{m}\left(t-\tau_{m}-(l-1) T_{c h}-(i-1) T_{s}\right)+n(t)
$$

where for example with the new notation $S_{1,1}^{(1)}(t)=S_{1}(t), S_{1,2}^{(1)}(t)=S_{2}(t), \ldots, S_{1, K_{1}}^{(1)}=$ $S_{K_{1}}(t)$ (for physical (=virtual) users of class 1 ), $S_{2,1}^{(1)}(t)=S_{K_{1}+1}(t), S_{2,1}^{(2)}(t)=S_{K_{1}+2}(t)$, $S_{2,2}^{(1)}(t)=S_{K_{1}+3}(t), S_{2,2}^{(2)}(t)=S_{K_{1}+4}(t), \ldots, S_{2, K_{2}}^{(2)}(t)=S_{K_{1}+2\left(K_{2}-1\right)+2}(t)$ (for all virtual users of all physical users of class 2 ).

Considering the reordering of virtual users, Fig. 3.4 displays the block diagram for the VPG multirate CDMA system, which is similar to Fig. 3.1 except that virtual users are considered instead of physical users, where the virtual users and their corresponding bits are defined/generated as illustrated in Fig. 3.2.

In an ideal situation (no channel effect), the user's spreading waveform is merely 


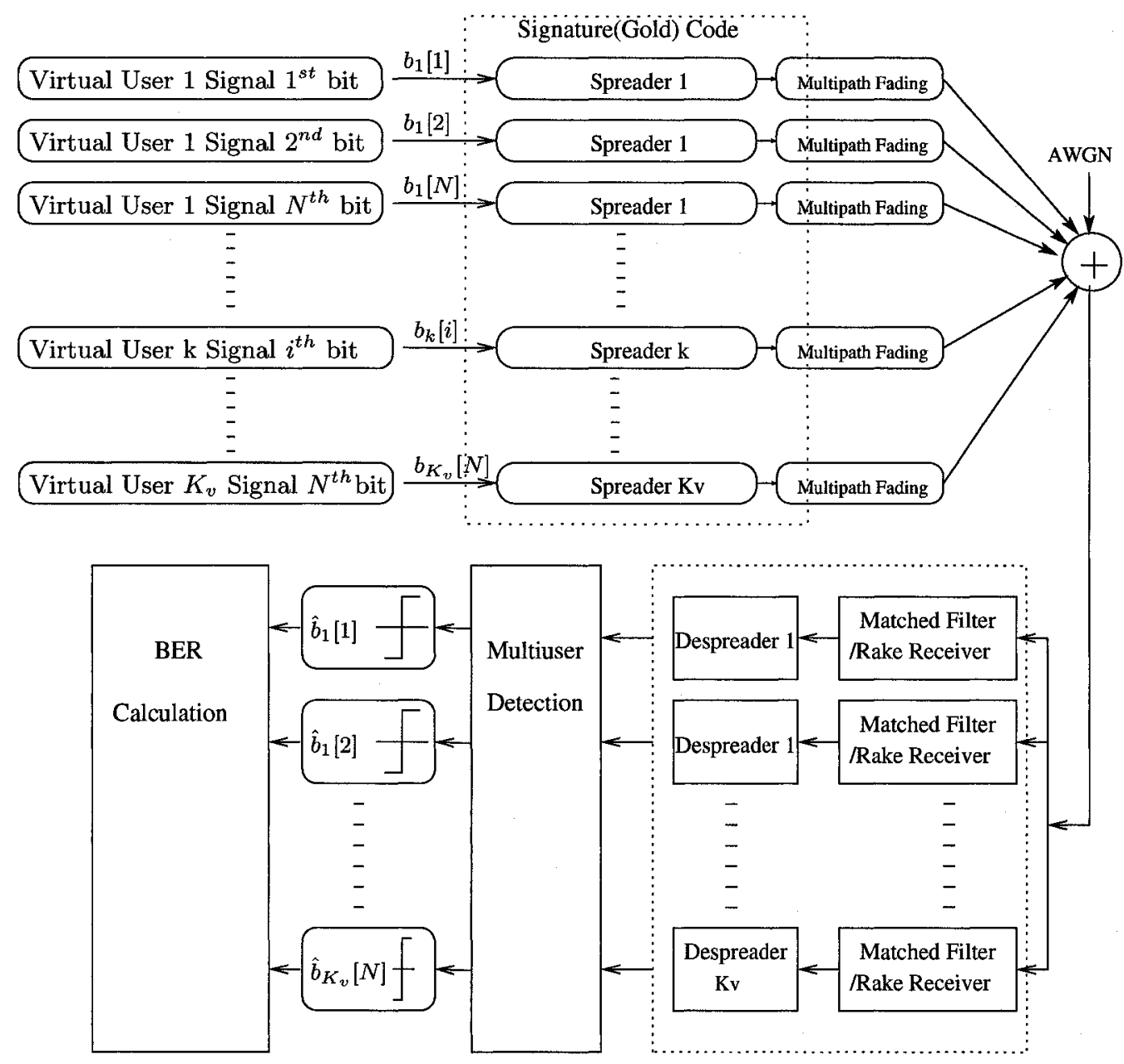

Fig. 3.4 Block diagram for the VPG multirate CDMA system

the convolution of its assigned code word and the pulse shaping filter. In the presence of multipath, the received signal also depends on the channel impulse response. Therefore, when the channel impulse is known to the receiver, it is convenient to express the received signal as a function of the user's effective signature waveform instead of its spreading waveform. The effective signature waveform depends on the user's spreading code and the channel impulse response as follows; Let $C_{m}(t)=\vec{\alpha}_{m}^{T} \cdot \vec{S}_{m}(t)$ be the effective signature sequence for the $m^{t h}$ virtual user, where $\vec{S}_{m}(t)=\left[S_{m}(t), S_{m}(t-\right.$ $\left.\left.T_{c h}\right), \cdots, S_{m}\left(t-(L-1) T_{c h}\right)\right]^{T}$ and $\vec{\alpha}_{m}=\left[\alpha_{m}[1], \alpha_{m}[2], \cdots, \alpha_{m}[L]\right]^{T}$. The received 
signal (3.9) can be re-written as:

$$
r(t)=\sum_{i=1}^{N} \sum_{m=1}^{K_{v}} \sqrt{E_{m}} b_{m}[i] C_{m}\left(t-\tau_{m}-(i-1) T_{s}\right)+n(t)
$$

\subsection{Proposed DBSIC receiver}

Multipath fading degrades the system's performance significantly. The RAKE receiver combats the effects of multipath by taking advantage of the inherent time diversity of multipath fading channels.

RAKE receiver: A RAKE receiver allows each arriving multipath signal to be individually demodulated and then combined to produce a stronger and more accurate signal [130]. It improves the quality of the received signal through processing of more than one of the multipath signals (use as much of the received energy as possible). The RAKE receiver uses a combination of correlators, code generators, and delays, or "fingers" that operate over each symbol interval (even if there is only one antenna), to spread out the individual echo signals of the multipath. Each signal is then delayed according to peaks found in the received signal. Each RAKE finger despreads a received signal component using an appropriately delayed code sequence. The same symbols obtained via different paths are then combined together using a combining scheme such as equal-gain-combining (EGC) or MRC where the correlator outputs are weighted so that the correlators responding to strong paths in the multipath environment have their energies counted, while the correlators not synchronizing with any significant path (outside the symbol interval) are suppressed or dispersed (e.g., MRC corrects channel phase rotation and weights components with channel amplitude estimates). The combined outputs are then sent to a simple decision device to decide on the transmitted symbols. 
RAKE receiver takes advantage of the inherent time diversity of multipath fading channels when the transmitting bandwidth is large enough to resolve the multipath components. It needs to know the multipath delays, phases of multipath components, amplitudes of multipath components and number of multipath components.

Rake principle can be used in DS-CDMA receivers to minimize the communication errors resulting from multipath effects. A coherent Rake receiver structure with MRC technique is implemented in this thesis with the number of RAKE fingers equal to the number of channel taps of the corresponding user being detected (Fig. 3.5).

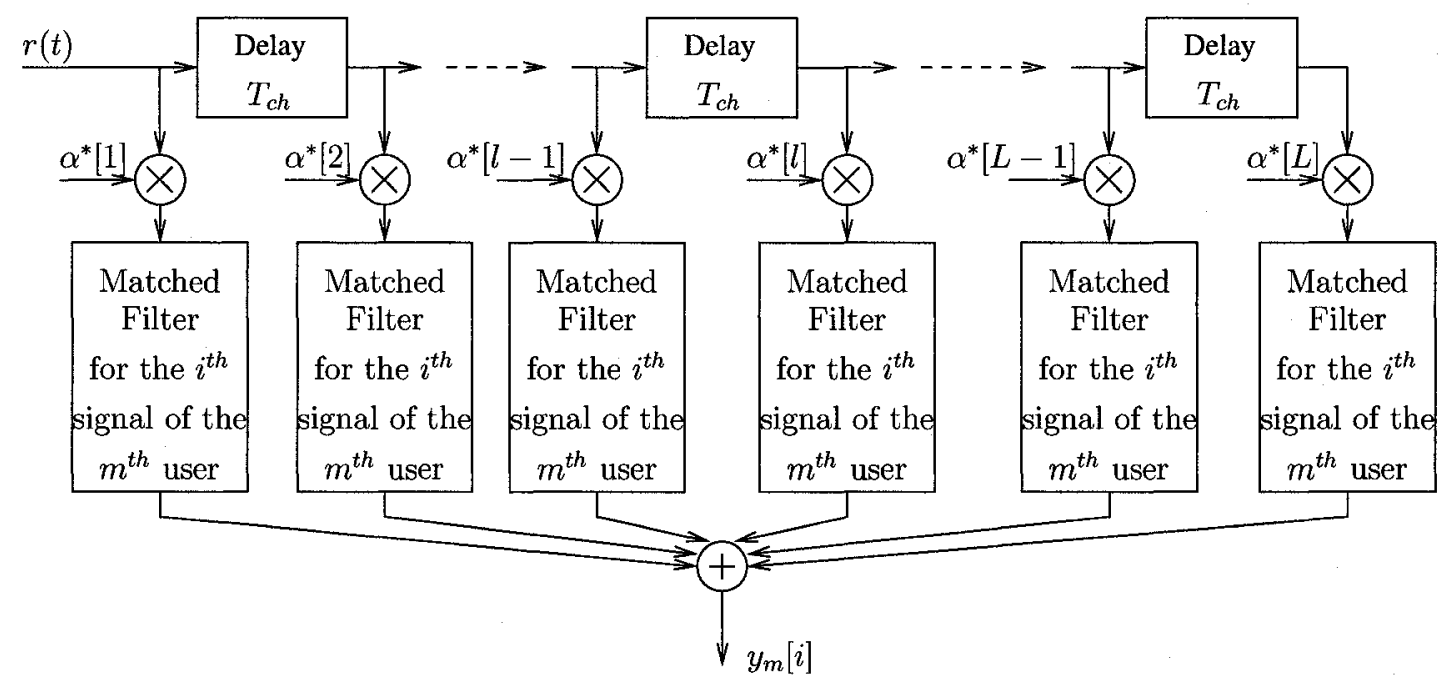

Fig. 3.5 RAKE receiver with MRC 
Output of a bank of $K_{v}$ RAKE matched filters: The output of the bank of $K_{v}$ Rake matched filters sampled at $T_{s}$ is given by:

$$
\begin{aligned}
y_{m}[i] & =\int_{(i-1) T_{s}+\tau_{m}}^{i T_{s}+\tau_{m}} r(t) C_{m}^{*}\left(t-\tau_{m}-(i-1) T_{s}\right) d t \\
& =\sum_{\check{i}=1}^{N} \sum_{\breve{m}=1}^{K_{v}}\left\{\sqrt{E_{\breve{m}}} b_{\breve{m}}[\check{i}] \int_{(i-1) T_{s}+\tau_{m}}^{i T_{s}+\tau_{m}} C_{\check{m}}\left(t-\tau_{\check{m}}-(\check{i}-1) T_{s}\right) C_{m}^{*}\left(t-\tau_{m}-(i-1) T_{s}\right)\right\} d t \\
& +\int_{(i-1) T_{s}+\tau_{m}}^{i T_{s}+\tau_{m}}\left\{n(t) C_{m}^{*}\left(t-\tau_{m}-(i-1) T_{s}\right)\right\} d t
\end{aligned}
$$

where $m=1, \ldots, K_{v}$ and $i=1, \ldots, N$. Let $\vec{y}[i]=\left[y_{1}[i], \cdots, y_{K_{v}}[i]\right]^{T}$, since $\tau_{m}<T_{s}$, the output $\vec{y}[i]$ can be written as $[131]$

$$
\vec{y}[i]=\mathbf{R}(-1) \mathbf{E} \vec{b}[i+1]+\mathbf{R}(0) \mathbf{E} \vec{b}[i]+\mathbf{R}(1) \mathbf{E} \vec{b}[i-1]+\vec{n}[i]
$$

where

$$
\begin{gathered}
\vec{b}[i]_{\left(K_{v} \times 1\right)}=\left[b_{1}[i], b_{2}[i], \cdots, b_{K_{v}}[i]\right]^{T} \\
\mathbf{E}_{\left(K_{v} \times K_{v}\right)}=\operatorname{diag}\left(\sqrt{E_{1}}, \sqrt{E_{2}}, \cdots, \sqrt{E_{K_{v}}}\right) \\
\vec{n}[i]_{\left(K_{v} \times 1\right)}=\left[n_{1}[i], n_{2}[i], \cdots, n_{K_{v}}[i]\right]^{T} \\
n_{m}[i]=\int_{(i-1) T_{s}+\tau_{m}}^{i T_{s}+\tau_{m}} n(t) C_{m}^{*}\left(t-\tau_{m}-(i-1) T_{s}\right) d t
\end{gathered}
$$

and $\mathbf{R}(-1), \mathbf{R}(0)$ and $\mathbf{R}(1)$ are $K_{v} \times K_{v}$ matrices defined as follows: 
The $(m n)^{t h}$ element $r_{m n}^{(0)}$ in $\mathbf{R}(0)$ is given by:

$$
\begin{aligned}
r_{m n}^{(0)} & =\int_{(i-1) T_{s}+\tau_{m}}^{i T_{s}+\tau_{m}}\left\{C_{n}\left(t-\tau_{n}-(i-1) T_{s}\right) C_{m}^{*}\left(t-\tau_{m}-(i-1) T_{s}\right)\right\} d t \\
& =\vec{\alpha}_{n}^{T} \int_{(i-1) T_{s}+\tau_{m}}^{i T_{s}+\tau_{m}}\left\{\vec{S}_{n}\left(t-\tau_{n}-(i-1) T_{s}\right) \vec{S}_{m}^{H}\left(t-\tau_{m}-(i-1) T_{s}\right)\right\} d t \vec{\alpha}_{m}^{*} \\
& =\vec{\alpha}_{n}^{T} \rho^{(0)}(n, m) \vec{\alpha}_{m}^{*}
\end{aligned}
$$

since $C_{n}(t)=\vec{\alpha}_{n}^{T} \cdot \vec{S}_{n}(t) . H$ denotes Hermitian conjugation, and $\rho^{(0)}(n, m)$ is an $L \times L$ matrix with $(k j)^{t h}$ element $\rho_{k j}^{(0)}(n, m)$ given by:

$\rho_{k j}^{(0)}(n, m)=\int_{(i-1) T_{s}+\tau_{m}}^{i T_{s}+\tau_{m}}\left\{\tau_{n}\left(t-(i-1) T_{s}-(k-1) T_{c h}\right) S_{m}^{*}\left(t-\tau_{m}-(i-1) T_{s}-(j-1) T_{c h}\right)\right\} d t$

The $(m n)^{t h}$ element $r_{m n}^{(1)}$ in $\mathbf{R}(1)$ is given by:

$$
\begin{aligned}
r_{m n}^{(1)} & =\int_{(i-1) T_{s}+\tau_{m}}^{i T_{s}+\tau_{m}}\left\{C_{n}\left(t-\tau_{n}-(i-2) T_{s}\right) C_{m}^{*}\left(t-\tau_{m}-(i-1) T_{s}\right)\right\} d t \\
& =\vec{\alpha}_{n}^{T} \int_{(i-1) T_{s}+\tau_{m}}^{i T_{s}+\tau_{m}}\left\{\vec{S}_{n}\left(t-\tau_{n}-(i-2) T_{s}\right) \vec{S}_{m}^{H}\left(t-\tau_{m}-(i-1) T_{s}\right)\right\} d t \vec{\alpha}_{m}^{*} \\
& =\vec{\alpha}_{n}^{T} \rho^{(\mathbf{1})}(\boldsymbol{n}, \boldsymbol{m}) \vec{\alpha}_{m}^{*}
\end{aligned}
$$

$\boldsymbol{\rho}^{(1)}(\boldsymbol{n}, \boldsymbol{m})$ is an $L \times L$ matrix with $(k j)^{t h}$ element $\rho_{k j}^{(1)}(n, m)$ given by:

$\rho_{k j}^{(1)}(n, m)=\int_{(i-1) T_{s}+\tau_{m}}^{i T_{s}+\tau_{m}}\left\{\tau_{n}\left(t-\tau_{n}-\left(i-T_{s}-(k-1) T_{c h}\right) S_{m}^{*}\left(t-\tau_{m}-(i-1) T_{s}-(j-1) T_{c h}\right)\right\} d t\right.$ 
The $(m n)^{t h}$ element $r_{m n}^{(-1)}$ in $\mathbf{R}(-1)$ is given by:

$$
\begin{aligned}
r_{m n}^{(-1)} & =\int_{(i-1) T_{s}+\tau_{m}}^{i T_{s}+\tau_{m}}\left\{C_{n}\left(t-\tau_{n}-i T_{s}\right) C_{m}^{*}\left(t-\tau_{m}-(i-1) T_{s}\right)\right\} d t \\
& =\vec{\alpha}_{n}^{T} \int_{(i-1) T_{s}+\tau_{m}}^{i T_{s}+\tau_{m}}\left\{\vec{S}_{n}\left(t-\tau_{n}-i T_{s}\right) \vec{S}_{m}^{H}\left(t-\tau_{m}-(i-1) T_{s}\right)\right\} d t \vec{\alpha}_{m}^{*} \\
& =\vec{\alpha}_{n}^{T} \rho^{(-1)}(\boldsymbol{n}, \boldsymbol{m}) \vec{\alpha}_{m}^{*}
\end{aligned}
$$

$\boldsymbol{\rho}^{(-1)}(\boldsymbol{n}, \boldsymbol{m})$ is an $L \times L$ matrix with $(k j)^{t h}$ element $\rho_{k j}^{(-1)}(n, m)$ given by:

$$
\rho_{k j}^{(-1)}(n, m)=\int_{(i-1) T_{s}+\tau_{m}}^{i T_{s}+\tau_{m}}\left\{S_{n}\left(t-\tau_{n}-i T_{s}-(k-1) T_{c h}\right) S_{m}^{*}\left(t-\tau_{m}-(i-1) T_{s}-(j-1) T_{c h}\right)\right\} d t
$$

In general, for $l=-1,0,1, \mathbf{R}(l)$ is a $K_{v} \times K_{v}$ matrix with $(m n)^{\text {th }}$ element $r_{m n}^{(l)}=$ $\vec{\alpha}_{n}^{T} \rho^{(l)}(\boldsymbol{n}, \boldsymbol{m}) \vec{\alpha}_{m}^{*}$ given by:

$$
\vec{\alpha}_{n}^{T} \rho^{(l)}(\boldsymbol{n}, \boldsymbol{m}) \vec{\alpha}_{m}^{*}=\int_{(i-1) T_{s}+\tau_{m}}^{i T_{s}+\tau_{m}} C_{n}\left(t-\tau_{n}-(i-1-l) T_{s}\right) C_{m}^{*}\left(t-\tau_{m}-(i-1) T_{s}\right) d t
$$

The matrix $\rho_{\boldsymbol{L} \times \boldsymbol{L}}^{(l)}(\boldsymbol{n}, \boldsymbol{m})$ has $(k j)^{t h}$ element $\rho_{k j}^{(l)}(n, m)$ :

$$
\begin{gathered}
\rho_{k j}^{(l)}(n, m)=\int_{(i-1) T_{s}+\tau_{m}}^{i T_{s}+\tau_{m}}\left[S_{n}\left(t-\tau_{n}-(i-1-l) T_{s}-(k-1) T_{c h}\right)\right. \\
\left.\cdot S_{m}^{*}\left(t-\tau_{m}-(i-1) T_{s}-(j-1) T_{c h}\right)\right] d t
\end{gathered}
$$

Considering the sequence of $N$ symbols, since $\tau_{m}<T_{s}$, from [131] the output $\vec{y}_{\left(N K_{v} \times 1\right)}=\left[\vec{y}^{T}[1], \vec{y}^{T}[2], \cdots \vec{y}^{T}[L]\right]^{T}$, with $\vec{y}[i]=\left[y_{1}[i], \cdots, y_{K_{v}}[i]\right]^{T}$ can be written as:

$$
\vec{y}=\overline{\mathbf{R}} \overline{\mathbf{E}} \vec{b}+\vec{n}
$$


where $\overline{\mathbf{R}}$ is a matrix of dimension $N K_{v} \times N K_{v}$

$$
\begin{aligned}
& \overline{\mathbf{R}}_{\left(N K_{v} \times N K_{v}\right)}=\left(\begin{array}{cccccc}
\mathbf{R}(0) & \mathbf{R}(-1) & 0 & 0 & \cdots & 0 \\
\mathbf{R}(1) & \mathbf{R}(0) & \mathbf{R}(-1) & 0 & \cdots & 0 \\
0 & \mathbf{R}(1) & \mathbf{R}(0) & \mathbf{R}(-1) & \ddots & 0 \\
\vdots & \ddots & \ddots & \ddots & \ddots & 0 \\
0 & 0 & \ddots & \mathbf{R}(1) & \mathbf{R}(0) & \mathbf{R}(-1) \\
0 & 0 & \cdots & 0 & \mathbf{R}(1) & \mathbf{R}(0)
\end{array}\right) \\
& \vec{y}_{\left(N K_{v} \times 1\right)}=\left[\vec{y}^{T}[1], \vec{y}^{T}[2], \cdots \vec{y}^{T}[N]\right]^{T} \vec{y}[i]=\left[y_{1}[i], \cdots, y_{K_{v}}[i]\right]^{T} \\
& \vec{b}_{\left(N K_{v} \times 1\right)}=\left[\vec{b}^{T}[1], \vec{b}^{T}[2], \cdots, \vec{b}^{T}[N]\right]^{T} \vec{b}[i]=\left[b_{1}[i], \cdots, b_{K_{v}}[i]\right]^{T} \\
& \overline{\mathbf{E}}_{\left(N K_{v} \times N K_{v}\right)}=\operatorname{diag}(\mathbf{E}, \mathbf{E}, \cdots, \mathbf{E}) \mathbf{E}=\operatorname{diag}\left(\sqrt{E_{1}}, \cdots, \sqrt{E_{K_{v}}}\right) \\
& \vec{n}_{\left(N K_{v} \times 1\right)}=\left[\vec{n}^{T}[1], \vec{n}^{T}[2], \cdots, \vec{n}^{T}[N]\right]^{T} \vec{n}[i]=\left[n_{1}[i], \cdots, n_{K_{v}}[i]\right]^{T}
\end{aligned}
$$

Rake Output after cancellation reordering Similarly to the conventional SIC and decorrelating DF schemes, for each sequence of length $N$, the received signal is passed through the bank of matched filters once and the DBSIC cancellation/detecting order is determined based on these outputs for all the symbols of the sequence. Let $\vec{y}_{n e w}$ be the vector obtained by reordering $\vec{y}$ so that its elements $y_{i}$ are listed in decreasing order. Then

$$
\vec{y}_{n e w}=\overline{\mathbf{R}}_{\text {new }} \overline{\mathbf{E}}_{\text {new }} \vec{b}_{n e w}+\vec{n}_{n e w}=\overline{\mathbf{Z}} \vec{b}_{n e w}+\vec{n}_{n e w}
$$




$$
y_{i}=\sum_{j=1}^{N K_{v}} z_{i j} b_{j}+n_{i} \quad i=1, \cdots, N K_{v}
$$

with $\overline{\mathbf{Z}}=\overline{\mathbf{R}}_{\text {new }} \overline{\mathbf{E}}_{\text {new }}$ and $b_{j}=j^{\text {th }}$ element of $\vec{b}_{\text {new }}$.

Proposed Decorrelator based SIC (DBSIC) receiver First step is to determine $b_{1}$, the first symbol of the strongest virtual user. Let

$$
\overline{\mathbf{Z}}^{(1)}=\overline{\mathbf{Z}}=\left(\begin{array}{ccccccc}
z_{11} & z_{12} & z_{13} & z_{14} & z_{15} & \cdots & z_{1 N K_{v}} \\
z_{21} & z_{22} & z_{23} & z_{24} & z_{25} & \cdots & z_{2 N K_{v}} \\
z_{31} & z_{32} & z_{33} & z_{34} & z_{35} & \cdots & z_{3 K_{v}} \\
\vdots & \vdots & \vdots & \vdots & \vdots & \vdots & \vdots \\
z_{N K_{v}-11} & z_{N K_{v}-12} & z_{N K_{v}-13} & z_{N K_{v}-14} & z_{N K_{v}-15} & \cdots & z_{N K_{v}-1 N K_{v}} \\
z_{N K_{v} 1} & z_{N K_{v} 2} & z_{N K_{v} 3} & z_{N K_{v} 4} & z_{N K_{v} 5} & \cdots & z_{N K_{v} N K_{v}}
\end{array}\right)
$$

Define $\vec{y}_{\text {new }}^{(1)}=\vec{y}_{\text {new }}=\left[y_{1}^{(1)}, y_{2}^{(1)}, y_{3}^{(1)}, y_{4}^{(1)}, y_{5}^{(1)}, \cdots y_{N K_{v}}^{(1)}\right]^{T}$, then

$$
\hat{b}_{1, \mathrm{DBSIC}}=\operatorname{sgn}\left(\Re\left\{\left[\overline{\mathbf{Z}}^{-\mathbf{1}} \vec{y}_{n e w}\right]_{1}\right\}\right)
$$

but unlike the decorrelating detector that detects all users' signals, only the first signal is determined at this stage. 
Second step is to detect $b_{2}$. Let

$$
\left\{\begin{array}{c}
y_{2}^{(2)}=y_{2}^{(1)}-z_{21} \hat{b}_{1} \\
\vdots \\
y_{i}^{(2)}=y_{i}^{(1)}-z_{i 1} \hat{b}_{1} \\
\vdots \\
y_{N K_{v}}^{(2)}=y_{N K_{v}}^{(1)}-z_{N K_{v} 1} \hat{b}_{1}
\end{array}\right.
$$

and $\vec{y}_{n e w}^{(2)}=\left[y_{2}^{(2)}, y_{3}^{(2)}, y_{4}^{(2)}, y_{5}^{(2)}, \cdots, y_{N K_{v}}^{(2)}\right]^{T}, \vec{b}_{n e w}^{(2)}=\left[b_{2}, b_{3}, b_{4}, b_{5}, \cdots, b_{N K_{v}}\right]^{T}, \vec{n}_{n e w}^{(2)}=$ $\left[n_{2}^{(2)}, n_{3}^{(2)}, n_{4}^{(2)}, n_{5}^{(2)}, \cdots, n_{N K_{v}}^{(2)}\right]^{T}$. We obtain

$$
\vec{y}_{\text {new }}^{(2)}=\overline{\mathbf{Z}}^{(2)} \vec{b}_{\text {new }}^{(2)}+\vec{n}_{\text {new }}^{(2)}
$$

where

$$
\overline{\mathbf{Z}}_{N K_{v}-1 \times N K_{v}-1}^{(2)}=\left(\begin{array}{cccccc}
z_{22} & z_{23} & z_{24} & z_{25} & \cdots & z_{2 N K_{v}} \\
z_{32} & z_{33} & z_{34} & z_{35} & \cdots & z_{3 N K_{v}} \\
\vdots & \vdots & \vdots & \vdots & \vdots & \vdots \\
z_{N K_{v}-12} & z_{N K_{v}-13} & z_{N K_{v}-14} & z_{N K_{v}-15} & \cdots & z_{N K_{v}-1 N K_{v}} \\
z_{N K_{v} 2} & z_{N K_{v} 3} & z_{N K_{v} 4} & z_{N K_{v} 5} & \cdots & z_{N K_{v} N K_{v}}
\end{array}\right)
$$

The estimate of $b_{2}$ is obtained using

$$
\hat{b}_{2}=\operatorname{sgn}\left(\Re\left\{\left[\left\{\overline{\mathbf{Z}}^{(\mathbf{2})}\right\}^{-1} \vec{y}_{\text {new }}^{(2)}\right]_{1}\right\}\right)
$$


Similarly, let

$$
\left\{\begin{array}{c}
y_{3}^{(3)}=y_{3}^{(2)}-z_{32} \hat{b}_{2} \\
\vdots \\
y_{i}^{(3)}=y_{i}^{(2)}-z_{i 2} \hat{b}_{2} \\
\vdots \\
y_{N K_{v}}^{(3)}=y_{N K_{v}}^{(2)}-z_{N K_{v} 2} \hat{b}_{2}
\end{array}\right.
$$

and follow the same procedure to detect the other $b_{i}$ 's $\left(b_{3}, \cdots, b_{k}, \cdots, b_{N K_{v}}\right)$. Generally, define $\vec{y}_{\text {new }}^{(k)}=\left[y_{k}^{(k)}, \cdots, y_{N K_{v}}^{(k)}\right]^{T}, \vec{n}_{\text {new }}^{(k)}=\left[n_{k}^{(k)}, \cdots, n_{N K_{v}}^{(k)}\right]^{T} \vec{b}_{n e w}^{(k)}=\left[b_{k}, \cdots, b_{N K_{v}}\right]^{T}$, where $y_{i}^{(1)} \triangleq y_{i}$, and for $i=k, \ldots, N K_{v}, k \geq 2$

$$
\begin{aligned}
y_{i}^{(k)} & \triangleq y_{i}^{(k-1)}-z_{i k-1} \hat{b}_{k-1}=\sum_{j=k}^{N K_{v}} z_{i j} b_{j}+n_{i}^{(k)} \\
n_{i}^{(k)} & \triangleq n_{i}+\sum_{j=1}^{k-1} z_{i j}\left(b_{j}-\hat{b}_{j}\right) \\
& \Longrightarrow \vec{y}_{\text {new }}^{(k)}=\overline{\mathbf{Z}}^{(\mathbf{k})} \vec{b}_{\text {new }}^{(k)}+\vec{n}_{\text {new }}^{(k)}
\end{aligned}
$$

where the matrix $\overline{\mathbf{Z}}_{\left(N K_{v}-k+1\right) \times\left(N K_{v}-k+1\right)}^{(\mathbf{k})}$ is given by:

$$
\left(\begin{array}{cccc}
z_{k k} & z_{k k+1} & \cdots & z_{k N K_{v}} \\
z_{k+1 k} & z_{k+1 k+1} & \cdots & z_{k+1 N K_{v}} \\
\vdots & \vdots & \vdots & \vdots \\
z_{N K_{v} k} & z_{N K_{v} k+1} & \cdots & z_{N K_{v} N K_{v}}
\end{array}\right)
$$

The estimate of $b_{k}$ is obtained using

$$
\hat{b}_{k, \text { DBSIC }}=\operatorname{sgn}\left(\Re\left\{\left[\left\{\overline{\mathbf{Z}}^{(\mathbf{k})}\right\}^{-1} \vec{y}_{\text {new }}^{(k)}\right]_{1}\right\}\right)
$$


Fig. 3.6 shows the receiver structure for DBSIC: As can be seen from Fig. 3.6,
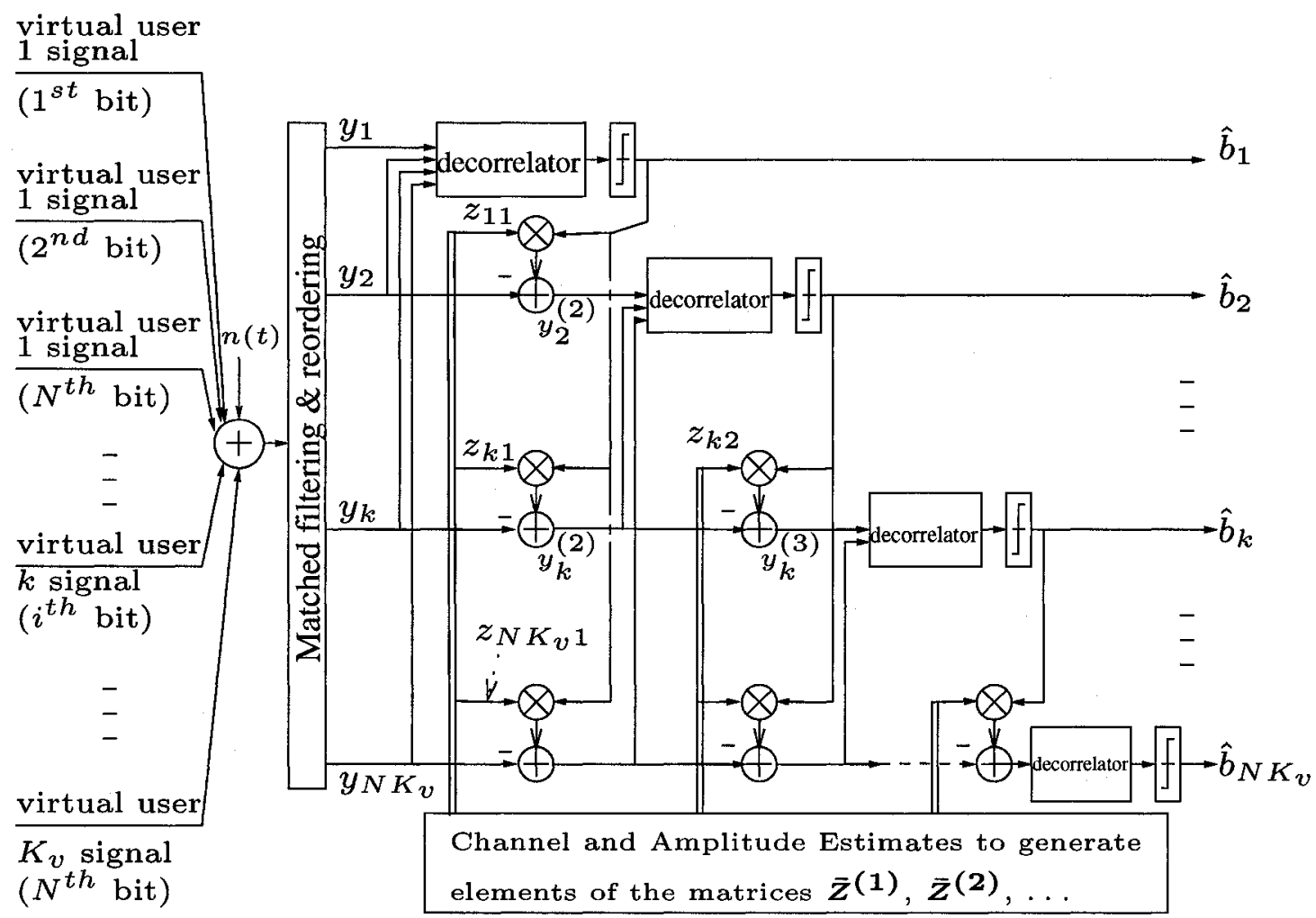

Fig. 3.6 DBSIC structure

DBSIC is similar to the conventional SIC detector except that a decorrelator is utilized on top of the conventional single-user MF detector to determine the signal estimate to be used for the users cancellation at the next stage, hence the named DecorrelatorBased SIC scheme.

\subsection{Commonly used multiuser detectors}

1. Conventional single-user MF detector:

$$
\hat{\vec{b}}_{\mathrm{MF}}=\operatorname{sgn}(\Re\{\vec{y}\})
$$


where $\Re\{\cdot\}$ = real part of · (elements by elements).

2. Decorrelating detector:

$$
\hat{\vec{b}}_{\mathrm{DEC}}=\operatorname{sgn}\left(\Re\left\{[\overline{\mathbf{R}} \overline{\mathbf{E}}]^{-1} \vec{y}\right\}\right)
$$

3. MMSE detector:

$$
\hat{\vec{b}}_{\mathrm{MMSE}}=\operatorname{sgn}\left(\Re\left\{\left[\overline{\mathbf{R}} \overline{\mathbf{E}}+\left(N_{0} / 2\right) \mathbf{I}\right]^{-1} \vec{y}\right\}\right)
$$

4. PIC detector: The decision statistic at stage $[\mathrm{g}]$ is:

$$
\hat{b}_{k, \mathrm{PIC}}^{(g)}=\operatorname{sgn}\left(\Re\left\{y_{k}^{\mathrm{MF}}-\sum_{j=1, j \neq k}^{N K_{v}} z_{k j}^{\mathrm{MF}} \hat{b}_{j, \mathrm{PIC}}^{(g-1)}\right\}\right) \begin{gathered}
k=1, \ldots, N K_{v} \\
\hat{b}_{j, \mathrm{PIC}}^{(0)}=0
\end{gathered}
$$

where $y_{k}^{\mathrm{MF}}=k^{\text {th }}$ element of $\vec{y}$ and $z_{k j}^{\mathrm{MF}}=k j^{\text {th }}$ element of $\overline{\mathbf{R}} \overline{\mathbf{E}}$.

For the conventional SIC and decorrelating DF, decision are obtained after the virtual users have been reordered, i.e. based on the elements of $\vec{y}_{n e w}$ given by (3.17).

5. Conventional SIC detector:

$$
\left\{\begin{aligned}
& \hat{b}_{1, \mathrm{SIC}}= \operatorname{sgn}\left(\Re\left\{y_{1}\right\}\right) \\
& \vdots \\
& \hat{b}_{k, \mathrm{SIC}}= \operatorname{sgn}\left(\Re\left\{y_{k}-\sum_{j=1}^{k-1} z_{k j} \hat{b}_{j, \mathrm{SIC}}\right\}\right) \\
& \vdots \\
& \hat{b}_{N K_{v}, \mathrm{SIC}}=\operatorname{sgn}\left(\Re\left\{y_{N K_{v}}-\sum_{j=1}^{N K_{v}-1} z_{N K_{v} j} \hat{b}_{j, \mathrm{SIC}}\right\}\right)
\end{aligned}\right.
$$


or equivalently

$$
\begin{aligned}
& \hat{b}_{1, \mathrm{SIC}}=\operatorname{sgn}\left(\Re\left\{y_{1}\right\}\right) \\
& \hat{b}_{k, \text { SIC }}=\operatorname{sgn}\left(\Re\left\{y_{k}-\sum_{j=1}^{k-1} z_{k j} \hat{b}_{j, \mathrm{SIC}}\right\}\right) \quad k=2, \ldots, N K_{v}
\end{aligned}
$$

6. Decorrelating DF detector: Let $\overline{\mathbf{Z}}=\mathbf{F}^{H} \mathbf{F}$, where $\mathbf{F}$ is a lower triangular matrix (obtained using techniques similar to the classical Cholesky decomposition). Applying $\mathbf{F}^{H^{-1}}$ (noise whitening filter) to $\vec{y}_{\text {new }}$ yields: $\quad \vec{y}_{f}=\mathbf{F} \vec{b}_{\text {new }}+\vec{n}_{f}$

$$
\hat{b}_{k, \mathrm{DF}}=\operatorname{sgn}\left(\Re\left\{y_{f, k}-\sum_{j=1}^{k-1} F_{k j} \hat{b}_{j, \mathrm{DF}}\right\}\right) \begin{gathered}
\hat{b}_{1, \mathrm{DF}}=\operatorname{sgn}\left(\Re\left\{y_{f, 1}\right\}\right) \\
k=2, \ldots, N K_{v}
\end{gathered}
$$

\subsection{Receivers'computational complexity}

In general one real addition is counted as one flop operation [55], one real multiplication is counted as one [55] or a few flop operations. Division and square root are more complicated and usually counted as multiple of flops. Therefore, in this work we count the number of multiplications, additions/subtractions, divisions and square roots separately in calculating the computational complexity. In the evaluation of the receivers complexity, we count only the operations after the matched filters' outputs (i.e. starting from (3.15)) [132]. The computational complexity for all the detection techniques used in this work are listed as below and detailed information can be found in appendix A.

1. Decorrelating:

$\left(N K_{v}\right)^{2}$ (divisions) $+\left(N K_{v}+1\right)\left(N K_{v}\right)^{2}$ (multiplications) $+\left[\left(N K_{v}\right)^{2}-1\right]\left(N K_{v}\right)$ (additions) 
If, similarly to [55] approach, we count the total number of multiplications and additions together, the complexity of the decorrelating detector is $\sim O\left[\left(N K_{v}\right)^{3}\right]$.

2. MMSE:

Implemented directly MMSE is more complex than the decorrelating detector (due to the additional additions before the matrix inversion), but its complexity can be reduced by using the LMS algorithm [55].

3. PIC with $g$ IC stages :

$$
g\left(N K_{v}-1\right) N K_{v}(\text { multiplications })+g\left(N K_{v}-1\right) N K_{v}(\text { additions/subtractions })
$$

and counting multiplications and additions together the total complexity is $\sim$ $O\left[g\left(N K_{v}\right)^{2}\right]$.

4. Conventional SIC:

$\frac{1}{2}\left(3 N K_{v}-1\right) N K_{v}($ multiplications $)+\left\{\frac{1}{2}\left(N K_{v}-1\right) N K_{v}+N K_{v} \log _{2}\left(N K_{v}\right)\right\}$ (additions) where the term $N K_{v} \log _{2}\left(N K_{v}\right)$ represents the complexity of the reordering of $\vec{y}$ to get $\vec{y}_{n e w}$ and has been treated as equivalent to $N K_{v} \log _{2}\left(N K_{v}\right)$ additions. Counting multiplications and additions together, the complexity is $\sim O\left[\left(N K_{v}\right)^{2}\right]$. 
5. Decorrelating DF:

$$
\begin{gathered}
\left(N K_{v}\right) \text { (square roots) }+\left(N K_{v}\right)^{2} \text { (divisions) } \\
+\frac{1}{3} N K_{v}\left[\left(N K_{v}\right)^{2}+6 N K_{v}-1\right](\text { multiplications }) \\
+\left\{\frac{1}{6} N K_{v}\left(N K_{v}-1\right)\left(2 N K_{v}+5\right)+N K_{v} \log _{2}\left(N K_{v}\right)\right\} \text { (additions) }
\end{gathered}
$$

Counting multiplications and additions together, the complexity is $\sim O\left[\left(N K_{v}\right)^{3}\right]$.

6. DBSIC:

$$
\begin{gathered}
\frac{1}{6} N K_{v}\left(N K_{v}+1\right)\left(2 N K_{v}+1\right)(\text { divisions }) \\
\left.+\frac{1}{12} N K_{v}\left[3\left(N K_{v}\right)^{3}+2\left(N K_{v}\right)^{2}+21 N K_{v}-2\right)\right] \text { (multiplications) } \\
+\left\{\frac{1}{12} N K_{v}\left[3\left(N K_{v}\right)^{3}+2\left(N K_{v}\right)^{2}+9 N K_{v}-2\right]+N K_{v} \log _{2}\left(N K_{v}\right)\right\} \text { (additions) }
\end{gathered}
$$

Counting multiplications and additions together, the complexity is $\sim O\left[\left(N K_{v}\right)^{4}\right]$. It is seen that DBSIC has a more complex structure than the other receivers due to the implementation of the decorrelator at each stage which translates into a repeated matrix inversion of decreasing dimension at each stage. However, with the implementation of iterative algorithms such as steepest descent (SD), conjugate gradient (CG), and preconditioned conjugate gradient (PCG) [18], the complexity and processing delay of DBSIC can be reduced.

This thesis investigates asynchronous multiuser multirate detection techniques. A novel multiuser multirate receiver (named DBSIC) has been proposed in section 3.3. The DBSIC scheme is similar to the conventional SIC scheme, except that at each stage of the SIC receiver, a decorrelator on top of conventional MF detector is employed to detect that user's signal. Then that new estimate is used for the 
next user cancellation stage. As will be seen in chapter 6, DBSIC yields improved performance over commonly-used multiuser receivers such as the decorrelating receiver under various multirate VPG scenarios (see for example Figs. 6.5-6.8). However, as seen in Section 3.5, the decorrelating receiver and DBSIC are computationally complex due to the presence of matrix inversion. Sections 3.6 and 3.7 present alternate multiuser multirate receiver structures to address the receiver complexity problem due to the presence of matrix inversion. Section 3.6 considers an implementation of the decorrelating detector with reduced complexity. Note since the decorrelating receiver and DBSIC require similar type of matrix inversion, the proposed algorithm in section 3.6 could be viewed as preliminary work towards the reduction of complexity of DBSIC. On the other hand, section 3.7 proposes an alternate receiver structure using PIC and decision feedback principles.

\subsection{Implementation of linear decorrelating detector with reduced complexity}

The linear decorrelating detector has a near-far resistance property and a linear complexity in the synchronous scenario. However, in the asynchronous case, the decorrelating detector is no longer near-far resistant and is computationally complicated. As mentioned in sections 2.1.2 and 2.1.3, there are two problems associated with using the decorrelating detector in an asynchronous scenario. The first one, similar to the synchronous case, is that the noise term in the matched filter bank output is no longer white due to the despreading process [2]. The second is related to the complexity of the inversion of the cross-correlation matrix due to its large size since in the asynchronous case it depends on both the number of users and the sequence length. One way to solve these problems is to use an equivalent white noise model obtained by 
passing the matched filter bank output through a pre-whitened filter. The challenge is the implementation of the ideal noise whitening filter. Various near ideal or approximate noise whitening filters have been proposed or examined [20]-[23]. We present a simple method to implement the ideal noise whitening filter directly by providing a novel adaptive algorithm that performs the cross-correlation matrices factorization. The introduced algorithm not only implements the ideal noise whitening filter, but also reduces the computational complexity that is related to the matrix inversion for the asynchronous decorrelating receiver.

As given by (3.10), the output of the bank of $K_{v}$ RAKE matched filters sampled at $T_{s}$ is written by:

$$
\vec{y}[i]=\mathbf{R}(-1) \mathbf{E} \vec{b}[i+1]+\mathbf{R}(0) \mathbf{E} \vec{b}[i]+\mathbf{R}(1) \mathbf{E} \vec{b}[i-1]+\vec{n}[i],
$$

where $\{\vec{n}[i]\}$ is a Gaussian vector with zero mean and autocorrelation matrix $E\left\{\vec{n}[i] \vec{n}^{H}[j] / 2\right\}=\frac{N_{0}}{2} \mathbf{R}(i-j)$. The noise term has an autocorrelation matrix as $\frac{N_{0}}{2} \mathbf{R}(i-j)$, thus is not white any more. An equivalent model is obtained by passing the received signal through a pre-whitened filter such that the noise term after filtering is white. The discrete-time model assuming no noise can be re-written in the $z$-transform domain as $[2]: \mathbf{S}(z)=\mathbf{R}^{T}[1] z+\mathbf{R}[0]+\mathbf{R}[1] z^{-1}=[\mathbf{F}[0]+\mathbf{F}[1] z]^{T}\left[\mathbf{F}[0]+\mathbf{F}[1] z^{-1}\right]$, where

$$
\begin{aligned}
& \mathbf{R}[0]=\mathbf{F}^{T}[0] \mathbf{F}[0]+\mathbf{F}^{T}[1] \mathbf{F}[1] \\
& \mathbf{R}[1]=\mathbf{F}^{T}[0] \mathbf{F}[1]
\end{aligned}
$$

$\mathbf{F}[0]$ is a lower triangular matrix, $\mathbf{F}[1]$ is an upper triangular matrix with zero diagonal entries, and $\left(\mathbf{F}^{T}[0]+\mathbf{F}^{T}[1] z^{-1}\right)^{-1}$ is the pre-whitened filter impulse response.

With the matched filter outputs passed through the filter $\left(\mathbf{F}^{T}[0]+\mathbf{F}^{T}[1] z^{-1}\right)^{-1}$, 
the output sequence becomes:

$$
\vec{y}_{w}[i]=\mathbf{F}(0) \mathbf{E} \vec{b}[i]+\mathbf{F}(1) \mathbf{E} \vec{b}[i-1]+\vec{n}_{w}[i]
$$

$\vec{n}_{w}(i)$ is a white Gaussian vector with zero mean and covariance matrix $\frac{N_{0}}{2} \mathbf{I}$.

The following recursive algorithm is proposed to obtain the matrices $\mathbf{F}[0]$ and $\mathbf{F}[1]$. Rewrite (3.27b) as:

$$
\mathbf{F}[1]=\left\{\mathbf{F}^{T}[0]\right\}^{-1} \mathbf{R}[1]
$$

then we can get

$$
\mathbf{F}^{T}[1]=\mathbf{R}^{T}[1]\left\{\mathbf{F}^{T}[0]^{-1}\right\}^{T}
$$

Plug $\mathbf{F}[1]$ and $\mathbf{F}^{T}[1]$ into (3.27a) yields:

$$
\mathbf{F}^{T}[0] \mathbf{F}[0]+\mathbf{R}^{T}[1]\left\{\mathbf{F}^{T}[0]^{-1}\right\}^{T}\left\{\mathbf{F}^{T}[0]\right\}^{-1} \mathbf{R}[1]=\mathbf{R}[\mathbf{0}]
$$

Let $\mathbf{Z}[\mathbf{0}]=\mathbf{F}^{T}[0] \mathbf{F}[0]$ which is a positive definite matrix. Equation (3.31) becomes

$$
\mathbf{Z}[0]+\mathbf{R}^{T}[1]\left\{\mathbf{F}^{T}[0]^{-1}\right\}^{T}\left\{\mathbf{F}^{T}[0]\right\}^{-1} \mathbf{R}[1]=\mathbf{R}[0]
$$

1. start from $\mathbf{Z}[0]=\mathbf{R}[0]$;

2. do Cholesky factorization on $\mathbf{Z}[0]$ to get $\mathbf{F}^{T}[0]$;

3. obtain $\left\{\mathbf{F}^{T}[0]\right\}^{-1}$ and plug into (3.32);

4. update $\mathbf{Z}[0]$ in (3.32), if $\left|\mathbf{Z}[0]-\mathbf{Z}[0]_{\text {previous }}\right|>$ convergence criteria, then go to 2nd step; otherwise stop and go to step 5;

5. take $\mathbf{F}[0]$ obtained in step 2 and find $\mathbf{F}[1]$ using (3.29); 
Let $\vec{y}_{\mathbf{w}}=\left[\vec{y}_{w}^{T}[1] \vec{y}_{w}^{T}[2] \cdots \vec{y}_{w}^{T}[N]\right]^{T}, \vec{b}_{\mathbf{w}}=\left[\vec{b}_{w}^{T}[1] \vec{b}_{w}^{T}[2] \cdots \vec{b}_{w}^{T}[N]\right]^{T}$, and $\mathbf{\mathbf { E }}$ is given by $(3.16 \mathrm{c}) . \quad \vec{n}_{\mathbf{w}}=\left[\vec{n}_{w}^{T}[1] \vec{n}_{w}^{T}[2] \cdots \vec{n}_{w}^{T}[N]\right]^{T}$, where $\vec{n}_{w}[i]$ is the white Gaussian vector with zero mean and covariance matrix $\frac{N_{0}}{2} \mathbf{I}$ present in (3.28). Using (3.28), the vector $\vec{y}_{\mathbf{w}}$ can be rewritten as:

$$
\vec{y}_{\mathbf{w}}=\mathbf{F} \overline{\mathbf{E}} \vec{b}_{\mathbf{w}}+\vec{n}_{\mathbf{w}}
$$

where $\mathbf{F}$ is given by

$$
\mathbf{F}_{\left(N K_{v} \times N K_{v}\right)}=\left(\begin{array}{ccccc}
\mathbf{F}(0) & 0 & 0 & \ldots & 0 \\
\mathbf{F}(1) & \mathbf{F}(0) & 0 & \ldots & 0 \\
\vdots & \ddots & \ddots & \ddots & \vdots \\
0 & 0 & \mathbf{F}(1) & \mathbf{F}(0) & 0 \\
0 & \ldots & 0 & \mathbf{F}(1) & \mathbf{F}(0)
\end{array}\right)
$$

For the decorrelating detector, the transmitted bits can be recovered by multiplying the inverse of $\mathbf{F} \overline{\mathbf{E}}$ with the vector $\vec{y}_{\mathbf{w}}$ and then taking the sign of each of the components. $\mathbf{F} \overline{\mathbf{E}}$ is a lower triangular matrix and there is a simple method to get the inverse. The estimate of the data can be easily obtained from $\hat{\vec{b}}_{\mathbf{w}}$, where $\hat{\vec{b}}_{\mathbf{w}}=\operatorname{sgn}\left(\Re\left\{[\mathbf{F} \overline{\mathbf{E}}]^{-\mathbf{1}} \vec{y}_{\mathbf{w}}\right\}\right)$.

The computational complexity for this method is obtained as:

$$
\begin{gathered}
3\left(K_{v}\right) \text { (square roots) }+\left\{\frac{1}{2}\left(N K_{v}+1\right) N K_{v}+3\left(K_{v}\right)^{2}\right\} \text { (divisions) } \\
+\left\{\frac{1}{6} N K_{v}\left[N K_{v}+1\right)\left(N K_{v}+2\right)+\frac{1}{2} K_{v}\left[5 K_{v}+1\right)\left(K_{v}-1\right)\right\} \text { (multiplications) } \\
+\left\{\frac{1}{6}\left[\left(K_{v}\right)^{2}-1\right] K_{v}+\frac{1}{2}\left(K_{v}-1\right)\left[5\left(K_{v}\right)^{2}-10 K_{v}+6\right]\right\} \text { (additions) }
\end{gathered}
$$

Comparing the decorrelating detector with the conventional method, it is observed this new method can reduce the computational complexity significantly when the 
length of transmitted bits is not very small $(N>3)$. The complexity for this method is $\sim O\left[\frac{1}{6}\left(N K_{v}\right)^{3}\right]$.

\subsection{Modified PIC}

This thesis investigates asynchronous multiuser multirate detection techniques. A novel multiuser multirate receiver (named DBSIC) combining SIC and decorrelating principles has been proposed in section 3.3. This section proposes another multiuser detection technique which is based on the same principles as multistage PIC but includes decision feedback as well.

Considering the same asynchronous VPG multi-rate CDMA system as for DBSIC (see section 3.3), the output of a bank of $K_{v}$ RAKE matched filters, matched to the effective signature sequence of each user, sampled at $T_{s}$ is given in matrix form as (or see also (3.15))

$$
\vec{y}=\overline{\mathbf{R}} \overline{\mathbf{E}} \vec{b}+\vec{n}
$$

where $\overline{\mathbf{R}}$ is a matrix of dimension $N K_{v} \times N K_{v}$ given by

$$
\overline{\mathbf{R}}_{\left(N K_{v} \times N K_{v}\right)}=\left(\begin{array}{cccccc}
\mathbf{R}(0) & \mathbf{R}(-1) & 0 & 0 & \ldots & 0 \\
\mathbf{R}(1) & \mathbf{R}(0) & \mathbf{R}(-1) & 0 & \ldots & 0 \\
0 & \mathbf{R}(1) & \mathbf{R}(0) & \mathbf{R}(-1) & \ddots & 0 \\
\vdots & \ddots & \ddots & \ddots & \ddots & 0 \\
0 & 0 & \ddots & \mathbf{R}(1) & \mathbf{R}(0) & \mathbf{R}(-1) \\
0 & 0 & \ldots & 0 & \mathbf{R}(1) & \mathbf{R}(0)
\end{array}\right)
$$


For $l=-1,0,1, \mathbf{R}(l)$ is a $K_{v} \times K_{v}$ matrix with $(m n)^{t h}$ element $r_{m n}^{(l)}$ given by:

$$
\begin{array}{rlr}
r_{m n}^{(l)} & =\int_{(i-1) T_{s}+\tau_{m}}^{i T_{s}+\tau_{m}} C_{n}\left(t-\tau_{n}-(i-1-l) T_{s}\right) C_{m}^{*}\left(t-\tau_{m}-(i-1) T_{s}\right) d t \\
\vec{y}_{\left(N K_{v} \times 1\right)} & =\left[\vec{y}^{T}[1], \vec{y}^{T}[2], \cdots, \vec{y}^{T}[N]\right]^{T} & \vec{y}[i]=\left[y_{1}[i], \cdots, y_{K_{v}}[i]\right]^{T} \\
\vec{b}_{\left(N K_{v} \times 1\right)} & =\left[\vec{b}^{T}[1], \vec{b}^{T}[2], \cdots, \vec{b}^{T}[N]\right]^{T} & \vec{b}[i]=\left[b_{1}[i], \cdots, b_{K_{v}}[i]\right]^{T} \\
\overrightarrow{\mathbf{E}}_{\left(N K_{v} \times N K_{v}\right)} & =\operatorname{diag}(\mathbf{E}, \mathbf{E}, \cdots, \mathbf{E}) & \mathbf{E}=\operatorname{diag}\left(\sqrt{E_{1}}, \cdots, \sqrt{E_{K_{v}}}\right) \\
\vec{n}_{\left(N K_{v} \times 1\right)} & =\left[\vec{n}^{T}[1], \vec{n}^{T}[2], \cdots, \vec{n}^{T}[N]\right]^{T} & \\
y_{m}[i] & \left.=\int_{(i-1) T_{s}+\tau_{m}}^{i T_{s}+\tau_{m}} r i\right]=\left[n_{1}[i], \cdots, n_{K_{v}}[i]\right]^{T} \\
n_{m}[i] & =\int_{(i-1) T_{s}+\tau_{m}}^{i T_{s}+\tau_{m}} n(t) C_{m}^{*}\left(t-\tau_{m}-(i-1) T_{s}\right) d t &
\end{array}
$$

Let $y_{i}^{\mathrm{MF}}, z_{i j}^{\mathrm{MF}}$ and $b_{i}^{\mathrm{MF}}$ be the elements of $\vec{y}, \overline{\mathbf{R}} \overline{\mathbf{E}}$ and $\vec{b}$ respectively, the conventional multistage PIC detector makes its decision based on

$$
\hat{b}_{k}^{(g)}=\operatorname{sgn}\left(\Re\left\{y_{k}^{\mathrm{MF}}-\sum_{j=1, j \neq k}^{N K_{v}} z_{k j}^{\mathrm{MF}} \hat{b}_{j}^{(g-1)}\right\}\right)
$$

where $\hat{b}_{k}^{(g-1)}$ is the estimate of the $k^{\text {th }}$ symbol from previous stage.

In this work, a new method is proposed to update the decision at the second stage 
with the latest $\hat{b}_{k}$ where:

$$
\left\{\begin{aligned}
\hat{b}_{1}^{(g)} & =\operatorname{sgn}\left(\Re\left\{y_{1}^{\mathrm{MF}}-\sum_{j=2}^{N K_{v}} z_{k j}^{\mathrm{MF}} \hat{b}_{j}^{(g-1)}\right\}\right) \\
\hat{b}_{2}^{(g)} & =\operatorname{sgn}\left(\Re\left\{y_{2}^{\mathrm{MF}}-\sum_{j=3}^{N K_{v}} z_{k j}^{\mathrm{MF}} \hat{b}_{j}^{(g-1)}-\hat{\lambda} z_{k 1}^{\mathrm{MF}} \hat{b}_{1}^{(g)}\right\}\right) \\
\vdots & \\
\hat{b}_{k}^{(g)} & =\operatorname{sgn}\left(\Re\left\{y_{k}^{\mathrm{MF}}-\sum_{j=k+1}^{N K_{v}} z_{k j}^{\mathrm{MF}} \hat{b}_{j}^{(g-1)}-\hat{\lambda} \sum_{j=1}^{k-1} z_{k j}^{\mathrm{MF}} \hat{b}_{j}^{(g)}\right\}\right) \\
\vdots &
\end{aligned}\right.
$$

In each stage of PIC (such as stage $g$ ), when detecting the $k^{\text {th }}$ symbol, the already detected results from $1^{\text {st }}$ to $(k-1)^{\text {th }}$ symbols at the current stage (stage $g$ ) are fed back to the detector by multiplying a feedback coefficient so that the system can make decision on the $k^{\text {th }}$ symbol in real-time and with more accurate results. The determination of the feedback coefficient $\hat{\lambda}$ is based on the comparison for these detected symbols between the decisions at the current stage $\left(\hat{b}_{1}^{(g)}, \ldots, \hat{b}_{k-1}^{(g)}\right)$ and decisions at the previous stage $\left(\hat{b}_{1}^{(g-1)}, \ldots, \hat{b}_{k-1}^{(g-1)}\right)$. If they are the same, then $\hat{\lambda}$ will be set as one. Otherwise, $\hat{\lambda}$ will be set as a pre-set value $\lambda$ which is less than one. This method is expected to improve the performance of the conventional multistage PIC scheme while keeping the same computational complexity. 


\section{Chapter 4}

\section{Joint Channel Estimation and Multiuser Detection}

\subsection{Introduction}

The task of a channel estimator is to estimate the fading channel vector given the matched filter output (received observation) and the transmitted data. Joint detection of all users is a means to improve the receiver's performance in DS-CDMA communication systems. Blind detection does not require knowledge of the channel but in some cases it is not the solution. Therefore, it is important to also investigate detection schemes that use knowledge of the channel parameters which are distinct for every user. The DBSIC scheme proposed in section 3.3 assumes perfect knowledge of the channel parameters.

In practical wireless communications systems, the channel coefficients are unknown to the receiver and the corresponding channel estimates are imperfect due to the existence of noise and interference. Due to MAI, conventional estimation of these parameters is not always reliable, which may lead to poor detection accuracy. DBSIC 
performance will be investigated under imperfect channel estimation assuming a model for the channel estimation errors in section 6.5. But it is also of interest to investigate the performance of DBSIC with imperfect channel estimates actually coming from a channel estimator, and to propose a joint channel estimation and multiuser detection scheme that would incorporate DBSIC principles.

There are several ways of estimating channel parameters. A commonly-used method, which can be referred to as data-aided channel estimation method, consists of sending a known sequence of symbols which is unique for a certain transmitter and which could be repeated in every transmission burst. These known bits could be available either as a preamble before the data or as bits in a separate pilot channel. Then the channel estimator is able to estimate the CIR or the channel parameters for each burst separately by exploiting the known transmitted bits and the corresponding received samples. The channel attenuations and delays are assumed to be constant during the estimation process. An alternate method, often used in tracking mode consists of using the previously detected symbols as "known symbols" to estimate channel parameters (decision-directed method). In this chapter, an adaptive MAI-resistant multiuser channel estimation scheme based on the combination of data-aided and decision-directed approaches with reasonable computational complexity that works jointly with the multiuser detection technique is developed for asynchronous multirate DS-CDMA systems. An integrated approach is taken such that channel estimation is coupled with data detection (DBSIC in particular). 


\subsection{Joint channel estimation and multiuser detection}

Similar to (3.8), the received signal at the receiver can be written as:

$$
\begin{aligned}
r(t)= & \sum_{i=1}^{N} \sum_{c=1}^{C} \sum_{k=1}^{K_{c}} \sum_{j=1}^{c} \sum_{l=1}^{L}\left\{\sqrt{E_{c, k}^{(j)}} b_{c, k}^{(j)}[i] \alpha_{c, k}^{(j)}[i, l] S_{c, k}^{(j)}\left(t-\tau_{c, k}^{(j)}-(l-1) T_{c h}-(i-1) T_{s}\right)\right\} \\
& +n(t)
\end{aligned}
$$

where $N^{1}$ represents either the number of the training symbols or the fed back transmission symbols, and we have assumed that the fading is constant over a symbol duration but may vary from symbol to symbol, hence the additional index $i$ in $\alpha_{c, k}^{(j)}[i, l]$. In other words, $\alpha_{c, k}^{(j)}[i, l]$ is the $l^{\text {th }}$ path gain of the $j^{\text {th }}$ virtual user derived from the $k^{\text {th }}$ physical user of class $c$ for the duration of the $i^{t h}$ transmitted symbol. $b_{c, k}^{(j)}[i]$ is the $i^{\text {th }}$ transmitted symbol for the $j^{\text {th }}$ virtual user derived from user $k$ in class $c, j=1, \cdots, c$ and $\in\{-1,1\} ; E_{c, k}^{(j)} / c$ is the transmitted signal energy of the $j^{\text {th }}$ virtual user derived from user $k$ in class $c, S_{c, k}^{(j)}(t)$ is the signature waveform of $j^{\text {th }}$ virtual user derived from user $k$ in class $c . T_{s}=T_{1, s}$ is the symbol duration for the slowest-class users, $\tau_{c, k}^{(j)}$ is the delay of the $j^{\text {th }}$ virtual user derived from user $k$ of class $c$. The noise $n(t)$ is a complex Gaussian random process with zero mean and power spectral density $\frac{N_{0}}{2}$.

Similar to (3.9), by ordering the virtual users, (4.1) can be simplified as

$$
r(t)=\sum_{i=1}^{N} \sum_{m=1}^{K_{v}} \sum_{l=1}^{L}\left\{\sqrt{E_{m}} b_{m}[i] \alpha_{m}[i, l] S_{m}\left(t-\tau_{m}-(l-1) T_{c h}-(i-1) T_{s}\right)\right\}+n(t)
$$

where $m$ has been defined as $m=\sum_{i=0}^{c-1} i K_{i}+c(k-1)+j ; k=1, \ldots, K_{c} ; j=1, \ldots, c$;

\footnotetext{
${ }^{1}$ Generally, the number of training symbols $N_{T}$ is different from the number of transmitted symbols $N_{\text {symbols }}\left(N_{T}<N_{\text {symbols }}\right)$, but the joint detector processes the signal similarly regardless if it is during the training mode (data-aided mode) or the decision-directed mode. For convenience we will use $N$ throughout this section.
} 
$c=1, \ldots, C$. But unlike (3.9) introduced for the DBSIC receiver structure, this expression does not consider the composite signature waveform since the fading for each path of each user is unique and has to be estimated separately.

Assuming that the users delays are known or have been previously estimated, and that all the spreading sequences of all users are known, the output of the bank of $K_{v}$ matched filters sampled at $T_{s}$ is given by:

$$
\begin{aligned}
y_{m}[i, l]= & \int_{(i-1) T_{s}+\tau_{m}+(l-1) T_{c h}}^{i T_{s}+\tau_{m}+(l-1) T_{c h}} r(t) S_{m}^{*}\left(t-\tau_{m}-(i-1) T_{s}-(l-1) T_{c h}\right) d t \\
= & \sum_{\check{i}=1}^{N} \sum_{\check{m}=1}^{K_{v}} \sum_{\check{l}=1}^{L}\left\{\sqrt{E_{\check{m}}} b_{\breve{m}}[\check{i}] \alpha_{\check{m}}[\check{i}, \check{l}] \int_{(i-1) T_{s}+\tau_{m}+(l-1) T_{c h}}^{i T_{s}+\tau_{m}+(l-1) T_{c h}}\right. \\
& \left.S_{\breve{m}}\left(t-\tau_{\check{m}}-(\check{i}-1) T_{s}-(\check{l}-1) T_{c h}\right) S_{m}^{*}\left(t-\tau_{m}-(i-1) T_{s}-(l-1) T_{c h}\right)\right\} d t \\
& +\int_{(i-1) T_{s}+\tau_{m}+(l-1) T_{c h}}^{i T_{s}+\tau_{m}+(l-1) T_{c h}} n(t) S_{m}^{*}\left(t-\tau_{m}-(i-1) T_{s}-(l-1) T_{c h}\right) d t \\
= & \sum_{u=-1}^{1} \sum_{\check{m}=1}^{K_{v}} \sum_{\check{l}=1}^{L} \sqrt{E_{\check{m}}} b_{\check{m}}[i-u] \alpha_{\check{m}}[i-u, \check{l}] r_{m \check{m}}^{l \grave{l}}(u)+n_{m}[i, l]
\end{aligned}
$$

where $r_{m \check{m}}^{\check{l}}(u)$ and $n_{m}[i, l]$ are defined as

$$
\begin{aligned}
r_{m \check{m}}^{l \check{l}}(u) & \left.=\int_{(i-1) T_{s}+\tau_{m}+(l-1) T_{c h}}^{i T_{s}+\tau_{m}+(l-1) T_{c h}} S_{\breve{m}}(t-u-1) T_{s}-(\check{l}-1) T_{c h}\right) S_{m}^{*}\left(t-\tau_{m}-(i-1) T_{s}-(l-1) T_{c h}\right) d t \\
& =\int_{\tau_{m}+(l-1) T_{c h}}^{\tau_{m}+(l-1) T_{c h}} S_{\breve{m}}\left(t-\tau_{\breve{m}}+u T_{s}-(\check{l}-1) T_{c h}\right) S_{m}^{*}\left(t-\tau_{m}-(l-1) T_{c h}\right) d t \\
n_{m}[i, l]= & \int_{(i-1) T_{s}+\tau_{m}+(l-1) T_{c h}}^{i T_{s}+\tau_{m}+(l-1) T_{c h}} n(t) S_{m}^{*}\left(t-\tau_{m}-(i-1) T_{s}-(l-1) T_{c h}\right) d t
\end{aligned}
$$


Let us define the $L \times L$ matrix $\mathbf{R}_{\mathbf{m n}}(\mathbf{u})_{(L \times L)}$ with $l \check{l}$ element $r_{m n}^{l \check{l}}(u)$, i.e.

$$
\mathbf{R}_{\mathbf{m n}}(\mathbf{u})_{(L \times L)}=\left(\begin{array}{cccc}
r_{m n}^{11}(u) & r_{m n}^{12}(u) & \cdots & r_{m n}^{1 L}(u) \\
r_{m n}^{21}(u) & r_{m n}^{22}(u) & \cdots & r_{m n}^{2 L}(u) \\
\vdots & \vdots & \vdots & \vdots \\
r_{m n}^{L 1}(u) & r_{m n}^{L 2}(u) & \cdots & r_{m n}^{L L}(u)
\end{array}\right)
$$

and

$$
\begin{aligned}
\vec{y}_{m}[i]_{(L \times 1)} & =\left[y_{m}[i, 1], y_{m}[i, 2], \cdots, y_{m}[i, L]\right]^{T} \\
\mathbf{E}_{\mathbf{m}(L \times L)} & =\operatorname{diag}\left(\sqrt{E_{m}}, \sqrt{E_{m}}, \cdots, \sqrt{E_{m}}\right) \\
\mathbf{b}_{\mathbf{m}}[\mathbf{i}]_{(L \times L)} & =\operatorname{diag}\left(b_{m}[i], b_{m}[i], \cdots, b_{m}[i]\right) \\
\vec{\alpha}_{m}[i]_{(L \times 1)} & =\left[\alpha_{m}[i, 1], \alpha_{m}[i, 2], \cdots, \alpha_{m}[i, L]\right]^{T} \\
\vec{n}_{m}[i]_{(L \times 1)} & =\left[n_{m}[i, 1], n_{m}[i, 2], \cdots, n_{m}[i, L]\right]^{T}
\end{aligned}
$$

Then (4.3) can be rewritten in vector form as

$$
\vec{y}_{m}[i]=\sum_{u=-1}^{1} \sum_{\check{m}=1}^{K_{v}} \mathbf{R}_{\mathbf{m} \check{\mathbf{m}}}(\mathbf{u}) \mathbf{E}_{\check{\mathbf{m}}} \mathbf{b}_{\check{\mathbf{m}}}[\mathbf{i}-\mathbf{u}] \vec{\alpha}_{\check{m}}[i-u]+\vec{n}_{m}[i]
$$

Let us define the $K_{v} L \times K_{v} L$ matrix $\mathbf{R}^{\mathbf{J}}(\mathbf{u})$ as

$$
\mathbf{R}^{\mathbf{J}}(\mathbf{u})_{\left(K_{v} L \times K_{v} L\right)}=\left(\begin{array}{cccc}
\mathbf{R}_{\mathbf{1 1}}(\mathbf{u}) & \mathbf{R}_{\mathbf{1 2}}(\mathbf{u}) & \cdots & \mathbf{R}_{\mathbf{1} \mathbf{K}_{\mathbf{v}}}(\mathbf{u}) \\
\mathbf{R}_{\mathbf{2 1}}(\mathbf{u}) & \mathbf{R}_{\mathbf{2 2}}(\mathbf{u}) & \cdots & \mathbf{R}_{\mathbf{2} \mathbf{K}_{\mathbf{v}}}(\mathbf{u}) \\
\vdots & \vdots & \vdots & \vdots \\
\mathbf{R}_{\mathbf{K}_{\mathbf{v}} \mathbf{1}}(\mathbf{u}) & \mathbf{R}_{\mathbf{K}_{\mathbf{v}} \mathbf{2}}(\mathbf{u}) & \cdots & \mathbf{R}_{\mathbf{K}_{\mathbf{v}} \mathbf{K}_{\mathbf{v}}}(\mathbf{u})
\end{array}\right)
$$


and

$$
\begin{aligned}
\vec{y}^{J}[i]_{\left(K_{v} L \times 1\right)} & =\left[\vec{y}_{1}^{T}[i], \vec{y}_{2}^{T}[i], \cdots, \vec{y}_{K_{v}}^{T}[i]\right]^{T} \\
\mathbf{E}_{\left(K_{v} L \times K_{v} L\right)}^{\mathbf{J}} & =\operatorname{diag}\left(\mathbf{E}_{\mathbf{1}}, \mathbf{E}_{\mathbf{2}}, \cdots, \mathbf{E}_{\mathbf{K}_{\mathbf{v}}}\right) \\
\mathbf{b}[\mathbf{i}]_{\left(K_{v} L \times K_{v} L\right)} & =\operatorname{diag}\left(\mathbf{b}_{1}[\mathbf{i}], \mathbf{b}_{\mathbf{2}}[\mathbf{i}], \cdots, \mathbf{b}_{\mathbf{K}_{\mathbf{v}}}[\mathbf{i}]\right) \\
\vec{\alpha}[i]_{\left(K_{v} L \times 1\right)} & =\left[\vec{\alpha}_{1}^{T}[i], \vec{\alpha}_{2}^{T}[i], \cdots, \vec{\alpha}_{K_{v}}^{T}[i]\right]^{T} \\
\vec{n}[i]_{\left(K_{v} L \times 1\right)} & =\left[\vec{n}_{1}^{T}[i], \vec{n}_{2}^{T}[i], \cdots, \vec{n}_{K_{v}}^{T}[i]\right]^{T}
\end{aligned}
$$

Then (4.4) can be written in vector form as

$$
\vec{y}^{J}[i]=\sum_{u=-1}^{1} \mathbf{R}^{\mathbf{J}}(\mathbf{u}) \mathbf{E}^{\mathbf{J}} \mathbf{b}[\mathbf{i}-\mathbf{u}] \vec{\alpha}[i-u]+\vec{n}[i]
$$

Then grouping the vectors $\vec{y}^{J}[i], \quad i=1, \ldots, N$ into one vector $\vec{y}^{J}=\left[\vec{y}^{J}[1]^{T}, \vec{y}^{J}[2]^{T}, \cdots, \vec{y}^{J}[N]^{T}\right]^{T},(4.5)$ can be written in vector form as

$$
\vec{y}^{J}=\overline{\mathbf{R}}^{\mathrm{J}} \overline{\mathbf{E}}^{\mathrm{J}} \overline{\mathbf{b}} \vec{\alpha}^{J}+\vec{n}
$$

where

$$
\begin{array}{r}
\vec{y}_{\left(N K_{v} L \times 1\right)}^{J}=\left[\vec{y}^{J}[1]^{T}, \vec{y}^{J}[2]^{T}, \cdots, \vec{y}^{J}[N]^{T}\right]^{T} ; \\
\vec{y}^{J}[i]_{\left(K_{v} L \times 1\right)}=\left[\vec{y}_{1}^{T}[i], \vec{y}_{2}^{T}[i], \cdots, \vec{y}_{K_{v}}^{T}[i]\right]^{T} ; \\
\vec{y}_{m}[i]_{(L \times 1)}=\left[y_{m}[i, 1], y_{m}[i, 2], \cdots, y_{m}[i, L]\right]^{T} ; \\
\overline{\mathbf{E}}_{\left(N K_{v} L \times N K_{v} L\right)}^{\mathbf{J}}=\operatorname{diag}\left(\mathbf{E}^{\mathbf{J}}, \mathbf{E}^{\mathbf{J}}, \cdots, \mathbf{E}^{\mathbf{J}}\right) ; \\
\mathbf{E}_{\left(K_{v} L \times K_{v} L\right)}^{\mathbf{J}}=\operatorname{diag}\left(\mathbf{E}_{\mathbf{1}}, \mathbf{E}_{\mathbf{2}}, \cdots, \mathbf{E}_{\mathbf{K}_{\mathbf{v}}}\right) ; \\
\mathbf{E}_{\mathbf{m}(L \times L)}=\operatorname{diag}\left(\sqrt{E_{m}}, \sqrt{E_{m}} \cdots, \sqrt{E_{m}}\right) ;
\end{array}
$$




$$
\begin{array}{r}
\overline{\mathbf{b}}_{\left(N K_{v} L \times N K_{v} L\right)}=\operatorname{diag}(\mathbf{b}[\mathbf{1}], \mathbf{b}[\mathbf{2}], \cdots, \mathbf{b}[\mathbf{N}]) ; \\
\mathbf{b}[\mathbf{i}]_{\left(K_{v} L \times K_{v} L\right)}=\operatorname{diag}\left(\mathbf{b}_{\mathbf{1}}[\mathbf{i}], \mathbf{b}_{\mathbf{2}}[\mathbf{i}], \cdots, \mathbf{b}_{\mathbf{K}_{\mathbf{v}}}[\mathbf{i}]\right) ; \\
\mathbf{b}_{\mathbf{m}}[\mathbf{i}](L \times L)=\operatorname{diag}\left(b_{m}[i], b_{m}[i] \cdots, b_{m}[i]\right) ; \\
\vec{\alpha}_{\left(N K_{v} L \times 1\right)}^{J}=\left[\vec{\alpha}^{T}[1], \vec{\alpha}^{T}[2], \cdots, \vec{\alpha}^{T}[N]\right]^{T} ; \\
\vec{\alpha}[i]_{\left(K_{v} L \times 1\right)}=\left[\vec{\alpha}_{1}^{T}[i], \vec{\alpha}_{2}^{T}[i], \cdots, \vec{\alpha}_{K_{v}}^{T}[i]\right]^{T} ; \\
\vec{\alpha}_{m}[i]_{(L \times 1)}=\left[\alpha_{m}[i, 1], \alpha_{m}[i, 2], \cdots, \alpha_{m}[i, L]\right]^{T} ; \\
\vec{n}_{\left(N K_{v} L \times 1\right)}=\left[\vec{n}^{T}[1], \vec{n}^{T}[2], \cdots, \vec{n}^{T}[N]\right]^{T} ; \\
\vec{n}[i]_{\left(K_{v} L \times 1\right)}=\left[\vec{n}_{1}^{T}[i], \vec{n}_{2}^{T}[i], \cdots, \vec{n}_{K_{v}}^{T}[i]\right]^{T} ; \\
\vec{n}_{m}[i]_{(L \times 1)}=\left[n_{m}[i, 1], n_{m}[i, 2], \cdots n_{m}[i, L]\right]^{T} ;
\end{array}
$$

and the matrix $\overline{\mathbf{R}}^{\mathrm{J}}$ has dimension of $N K_{v} L \times N K_{v} L$ and has a similar form as $\overline{\mathbf{R}}$ in section 3.3:

$$
\overline{\mathbf{R}}_{\left(N K_{v} L \times N K_{v} L\right)}^{\mathbf{J}}=\left(\begin{array}{cccccc}
\mathbf{R}^{\mathbf{J}}(0) & \mathbf{R}^{\mathbf{J}}(-1) & 0 & 0 & \cdots & 0 \\
\mathbf{R}^{\mathbf{J}}(1) & \mathbf{R}^{\mathbf{J}}(0) & \mathbf{R}^{\mathbf{J}}(-1) & 0 & \ldots & 0 \\
0 & \mathbf{R}^{\mathbf{J}}(1) & \mathbf{R}^{\mathbf{J}}(0) & \mathbf{R}^{\mathbf{J}}(-1) & \ddots & 0 \\
\vdots & \ddots & \ddots & \ddots & \ddots & 0 \\
0 & 0 & \ddots & \mathbf{R}^{\mathbf{J}}(1) & \mathbf{R}^{\mathbf{J}}(0) & \mathbf{R}(-1) \\
0 & 0 & \cdots & 0 & \mathbf{R}^{\mathbf{J}}(1) & \mathbf{R}^{\mathbf{J}}(0)
\end{array}\right)
$$

Note that the known pilot symbols sequence $\overline{\mathbf{b}}$ is written in diagonal matrix form while the unknown channel side information $\vec{\alpha}^{J}$ is in vector form.

The task of a channel estimator is to estimate the fading vector $\vec{\alpha}^{J}$ in (4.6) given the received observation $\vec{y}^{J}$ and the transmitted data $\overline{\mathrm{b}}$. The ML algorithm is shown to be efficient for estimating multipath Rayleigh fading channels when the underlying 
channels are completely unknown and when the fading processes are slow. The ML estimates of the parameter vector $\vec{\alpha}^{J}$ can be expressed as:

$$
\hat{\vec{\alpha}}^{J}=\left(\overline{\mathbf{R}}^{\mathbf{J}} \overline{\mathbf{E}}^{\mathbf{J}} \overline{\mathbf{b}}\right)^{-1} \vec{y}^{J}
$$

where similar to $\vec{\alpha}^{J}, \hat{\vec{\alpha}}^{J}$ can be written as

$$
\begin{array}{r}
\hat{\vec{\alpha}}_{\left(N K_{v} L \times 1\right)}^{J}=\left[\hat{\vec{\alpha}}^{T}[1], \hat{\vec{\alpha}}^{T}[2], \cdots, \hat{\vec{\alpha}}^{T}[N]\right]^{T} ; \\
\hat{\vec{\alpha}}[i]_{\left(K_{v} L \times 1\right)}=\left[\hat{\vec{\alpha}}_{1}^{T}[i], \hat{\vec{\alpha}}_{2}^{T}[i], \cdots, \hat{\vec{\alpha}}_{K_{v}}^{T}[i]\right]^{T} ; \\
\hat{\vec{\alpha}}_{m}[i]_{(L \times 1)}=\left[\hat{\alpha}_{m}[i, 1], \hat{\alpha}_{m}[i, 2], \cdots, \hat{\alpha}_{m}[i, L]\right]^{T} ;
\end{array}
$$

Because the channel estimation includes added noise, filtering might be needed for reducing the noise. In other words the channel estimates can be smoothened by using an FIR filter. However, this filtering operation causes delay in the channel estimation process.

As the matrix dimension for inversion in (4.8) is obviously larger than that in the multiuser detection process and the direct inversion may need a significant amount of computing time and memory storage, techniques not involving direct matrix inversion could be used. For example, the LMS adaptive method can be implemented for the channel estimation process in (4.8). Some other adaptation rules like RLS could also be considered. These could improve the speed of convergence, but on the other hand are computationally more complex than LMS.

In the case of a slowly fading channel, $\vec{\alpha}[1]=\vec{\alpha}[2]=\cdots=\vec{\alpha}[N]$. Furthermore, all the virtual users derived from the same physical user undergo the same channel, so there are only $K L$ physical channel paths that need to be estimated where $K=$ $\sum_{c=1}^{C} K_{c}$ is the total number of physical users. Therefore, we can use these properties 
to improve the estimates of the channel fading coefficients estimates obtained from (4.8).

For convenience, let us define two new vectors:

$\hat{\vec{\alpha}}_{p}$ and $\hat{\vec{\alpha}}_{v}$ are the estimates of the channel vectors for the paths of all the physical and virtual users with the dimension of $K L \times 1$ and $K_{v} L \times 1$ respectively.

Using the fact that $\vec{\alpha}[1]=\vec{\alpha}[2]=\cdots=\vec{\alpha}[N]$, an estimate of the channel vector for the paths of all the virtual users in the case of a slowly fading channel can be obtained as

$$
\hat{\vec{\alpha}}_{v}=\frac{1}{N} \sum_{i=1}^{N} \hat{\vec{\alpha}}[i]
$$

$\hat{\vec{\alpha}}_{v}$ can be written as

$$
\begin{array}{r}
\hat{\vec{\alpha}}_{v\left(K_{v} L \times 1\right)}=\left[\hat{\vec{\alpha}}_{v, 1}^{T}, \hat{\vec{\alpha}}_{v, 2}^{T}, \cdots, \hat{\vec{\alpha}}_{v, K_{v}}^{T}\right]^{T} ; \\
\hat{\vec{\alpha}}_{v, m(L \times 1)}=\left[\hat{\alpha}_{v, m}[1], \hat{\alpha}_{v, m}[2], \cdots, \hat{\alpha}_{v, m}[L]\right]^{T} ;
\end{array}
$$

where for every $l=1, \ldots, L, m=1, \ldots, K_{v}, \hat{\alpha}_{v, m}[l]$ is an improved estimate of $\alpha_{m}[i, l]$ (regardless of the value of $i \in\{1, \ldots, N\})$ compared to $\hat{\alpha}_{m}[i, l]$ obtained from $(4.9 \mathrm{c}$ ) and (4.8).

Considering the ordering of the virtual users used (e.g. $m=\sum_{i=0}^{c-1} i K_{i}+c(k-1)+j$, $\left.k=1, \ldots, K_{c} ; j=1, \ldots, c ; c=1, \ldots, C\right)$, an estimate of the channel vector for the paths of all the physical users can be obtained as

$$
\begin{array}{r}
\hat{\vec{\alpha}}_{p(K L \times 1)}=\left[\hat{\vec{\alpha}}_{p, 1}^{T}, \hat{\vec{\alpha}}_{p, 2}^{T}, \cdots, \hat{\vec{\alpha}}_{p, K}^{T}\right]^{T} ; \\
\hat{\vec{\alpha}}_{p, r(L \times 1)}=\left[\hat{\alpha}_{p, r}[1], \hat{\alpha}_{p, r}[2], \cdots \hat{\alpha}_{p, r}[L]\right]^{T} ;
\end{array}
$$


where for $l=1, \ldots, L$

$$
\left\{\begin{array}{rlrl}
\hat{\alpha}_{p, r}[l] & =\hat{\alpha}_{v, r}[l], & 1 \leq r \leq K_{1} \\
\hat{\alpha}_{p, r}[l] & =\frac{1}{2}\left(\hat{\alpha}_{v, 2 r-K_{1}-1}[l]+\hat{\alpha}_{v, 2 r-K_{1}}[l]\right), & & K_{1}+1 \leq r \leq K_{1}+K_{2} \\
\vdots & & \\
\hat{\alpha}_{p, r}[l]=\frac{1}{c} \sum_{i=1}^{c} \hat{\alpha}_{v, c r-\sum_{k=1}^{c-1}(c-k) K_{k}+i-c}[l], & & \sum_{i=1}^{c-1} K_{i}+1 \leq r \leq \sum_{i=1}^{c} K_{i} \\
\vdots & & \\
\hat{\alpha}_{p, r}[l]=\frac{1}{C} \sum_{i=1}^{C} \hat{\alpha}_{v, C r-\sum_{k=1}^{C-1}(C-k) K_{k}+i-C}[l], & & \sum_{i=1}^{C-1} K_{i}+1 \leq r \leq \sum_{i=1}^{C} K_{i}
\end{array}\right.
$$

Once the channel estimated information are obtained for the physical users, they are used to generate an estimate of the channel information for the virtual users $\left\{\hat{\alpha}_{v, m}\right\}_{m=1, \ldots, K_{v}}$ (which will be used by the multiuser detection) as follows:

$$
\left\{\begin{array}{cc}
\hat{\alpha}_{v, m}[l]=\hat{\alpha}_{p, m}[l], & 1 \leq m \leq K_{1} \\
\hat{\alpha}_{v, m}[l]=\hat{\alpha}_{p,\left\lfloor\frac{m+K_{1}+1}{2}\right\rfloor}[l], & K_{1}+1 \leq m \leq K_{1}+2 K_{2} \\
\vdots & \\
\hat{\alpha}_{v, m}[l]=\hat{\alpha}_{p,\left\lfloor\frac{m+\sum_{k=1}^{c-1}(c-k) K_{k}-1+c}{c}\right\rfloor}[l], & \sum_{i=1}^{c-1} i K_{i}+1 \leq m \leq \sum_{i=1}^{c} i K_{i} \\
\vdots & \\
\hat{\alpha}_{v, m}[l]=\hat{\alpha}_{p,\left\lfloor\frac{m+\sum_{k=1}^{C-1}(C-k) K_{k}-1+C}{C}\right\rfloor}[l], & \sum_{i=1}^{C-1} i K_{i}+1 \leq m \leq \sum_{i=1}^{C} i K_{i}
\end{array}\right.
$$

where $\lfloor x\rfloor$ is the integer part of $x$ (ex. $\left\lfloor\frac{3}{2}\right\rfloor=1$ ). 
The proposed iterative joint channel estimation and multiuser detection scheme works then as follows:

- Step 1: Estimate the channel information for the virtual users from (4.14) upon receiving the training symbol sequence. Note that in fact first (4.8) is calculated, then (4.10), followed by (4.13) and finally (4.14).

- Step 2: Using DBSIC scheme described in section 3.3, detect the symbol sequence of the virtual users (which can be directly translated in symbol sequences of the physical users taking into consideration the various user rates).

- Step 3: The estimates of the symbols of the virtual users are fedback to the channel estimator to be considered as new training symbols. Assuming that the channel is time-invariant (slow fading) for the duration of the training sequence plus the transmitted sequence, new channel estimates ${ }^{2}$ are obtained according to step 1 but with the detected symbols as a training sequence.

- Step 4: Using the DBSIC scheme described in Section 3.3, redetect the symbol sequence of the virtual users using the new channel estimates ${ }^{3}$ (from step 3).

- Step 5: Repeat steps 3-4 iteratively.

In other words, the channel estimation is carried out iteratively where each channel estimation is followed by DBSIC detection employing the latest channel estimates. And the decisions obtained by DBSIC are in turn used to reduce the channel estimation error. Steps 1-4 constitutes one iteration, any subsequent iteration would involve only

\footnotetext{
${ }^{2}$ For sufficiently large SNR such that decision errors are small, step 3 is expected to yield improved channel estimates compared to those obtained in step 1.

${ }^{3}$ When improved channel estimates compared to those obtained in step 1 are obtained, step 4 is expected to yield improved estimates of the symbol sequences compared to step 2 .
} 
steps 3-4. Such joint iterative channel estimation and multiuser (DBSIC) detection scheme is illustrated in Fig. 4.1.

The main complexity of the algorithm lies in the matrix inversion in (4.8) which can be reduced considerably by using LMS or by using the fact that the matrix $\overline{\boldsymbol{R}}^{J}$ has a special form with a significant number of zeros. Therefore, the proposed algorithm including (4.10), (4.13) and (4.14) is expected to be simple enough to be suitable for practical implementation.

The effectiveness of the channel estimator will be demonstrated in section 6.6 in terms of the normalized MSE of the channel fading estimates of the virtual users obtained from (4.14) as well as the BER performance of a ten-user multirate CDMA system that employs the proposed joint channel estimation and multiuser detection algorithm.

We define the normalized MSE as $E\left[\left\{\vec{\alpha}_{v}-\hat{\vec{\alpha}}_{v}\right\}^{H}\left\{\vec{\alpha}_{v}-\hat{\vec{\alpha}}_{v}\right\}\right] / E\left[\vec{\alpha}_{v}^{H} \vec{\alpha}_{v}\right\}$, where the elements of $\hat{\vec{\alpha}}_{v}$ (see (4.11)) are obtained from (4.14). 


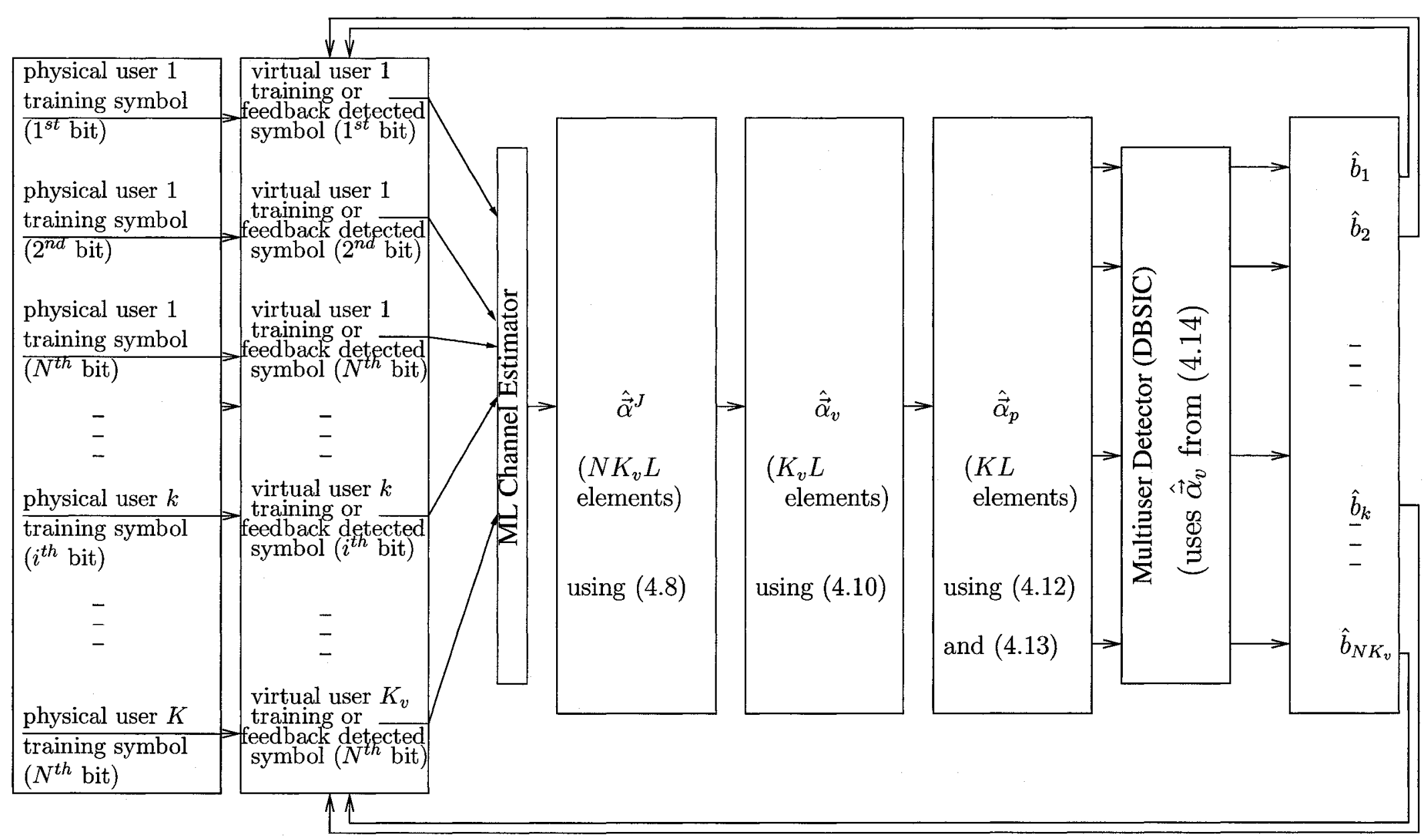

Fig. 4.1 Channel Estimator structure 


\section{Chapter 5}

\section{Analytical Performance Analysis}

\subsection{BER performance analysis}

Analysis for DBSIC Let $\mathbf{V}^{(k)}=\left\{\overline{\mathbf{Z}}^{(\mathbf{k})}\right\}^{-1}$, substituting (3.24) into (3.25) yields

$$
\begin{aligned}
\hat{b}_{k, \mathrm{DBSIC}} & =\operatorname{sgn}\left(\Re\left\{\left[\vec{b}_{\text {new }}^{(k)}+\mathbf{V}^{(k)} \vec{n}_{\text {new }}^{(k)}\right]_{1}\right\}\right)=\operatorname{sgn}\left(\Re\left\{b_{k}+\sum_{i=1}^{N K_{v}-k+1} v_{1 i}^{(k)} n_{k+i-1}^{(k)}\right\}\right) \\
& =\operatorname{sgn}\left(b_{k}+\Re\left\{\sum_{i=1}^{N K_{v}-k+1} v_{1 i}^{(k)} n_{i+k-1}\right\}+\sum_{i=1}^{N K_{v}-k+1} \sum_{j=1}^{k-1} \Re\left\{v_{1 i}^{(k)} z_{i+k-1 j}\right\}\left(b_{j}-\hat{b}_{j}\right)\right)
\end{aligned}
$$

In this section the performance of DBSIC and conventional SIC is analyzed by treating all the residual interference (residual MAI and residual noise component) in (5.1)[43, 101]. Let us assume further that the residual MAI is uncorrelated with the noise at the soft detector output (i.e. before the threshold stage). Then the probability of error for the $k^{\text {th }}$ virtual user, conditioned on the knowledge of the channel coefficients can be obtained as a Q-function. And the conditional probability of error for the system can be obtained by taking the average of all virtual users. The final error probability is obtained by averaging over the channel coefficients. 
Following [43] we assume that $\Re\left\{\sum_{i=1}^{N K_{v}-k+1} v_{1 i}^{(k)} n_{i+k-1}\right\}$ and $\left(b_{j}-\hat{b}_{j}\right)$ are independent Gaussian random variables. Since all terms of the summation are independent, $\Re\left\{\sum_{i=1}^{N K_{v}-k+1} v_{1 i}^{(k)} n_{k+i-1}^{(k)}\right\}$ has zero mean, and its variance is given by the sum of the variances of the two terms.

$$
\begin{aligned}
& \operatorname{Var}\left[\Re\left\{\sum_{i=1}^{N K_{v}-k+1} v_{1 i}^{(k)} n_{k+i-1}^{(k)}\right\} \mid \vec{\alpha}\right] \\
& =\operatorname{Var}\left[\Re\left\{\sum_{i=1}^{N K_{v}-k+1} v_{1 i}^{(k)} n_{i+k-1}\right\} \mid \vec{\alpha}\right]+\operatorname{Var}\left[\sum_{i=1}^{N K_{v}-k+1} \sum_{j=1}^{k-1} \Re\left\{v_{1 i}^{(k)} z_{i+k-1 j}\right\}\left(\hat{b}_{j}-b_{j}\right) \mid \vec{\alpha}\right]
\end{aligned}
$$

Assuming uncorrelated zero mean decision errors, using $E\left[n_{k} n_{l}^{*} / 2 \mid \vec{\alpha}\right]=\frac{N_{0}}{2}\left[\overline{\mathbf{R}}_{\text {new }}\right]_{k l}=$ $\frac{N_{0}}{2 \epsilon_{l}} z_{k l}$. and the Gaussian assumption of [43], it can be obtained that (see Appendix B)

$$
P_{e, k \mid \vec{\alpha}, \text { DBSIC }} \approx Q\left(\left[v_{11}^{(k)} \frac{N_{0}}{2 \epsilon_{k}}+4 \sum_{j=1}^{k-1}\left(\Re\left\{\sum_{i=1}^{N K_{v}-k+1} v_{1 i}^{(k)} z_{i+k-1 j}\right\}\right)^{2} P_{e, j \mid \vec{\alpha}}\right]^{-\frac{1}{2}}\right)
$$

where $P_{e, 1 \mid \vec{\alpha}, \mathrm{DBSIC}}=Q\left(\left[v_{11}^{(1)} \frac{N_{0}}{2 \epsilon_{1}}\right]^{-\frac{1}{2}}\right)$ and $\epsilon_{k}$ denotes the $k k^{t h}$ element of the diagonal matrix $\overline{\mathbf{E}}_{\text {new }}$. The average system BER performance is then obtained by averaging $P_{e \mid \vec{\alpha}, \text { DBSIC }}=\frac{1}{N K_{v}} \sum_{k=1}^{N K_{v}} P_{e, k \mid \vec{\alpha}, \text { DBSIC }}$ over the joint pdf of $\vec{\alpha}$.

Analysis for SIC : The expression of the conditional BER for the conventional SIC can be obtained using the same technique and it is shown to be (see Appendix C)

$$
P_{e, k \mid \vec{\alpha}, \mathrm{SIC}} \approx Q\left(\left[z_{k k}^{-1} \frac{N_{0}}{2 \epsilon_{k}}+\sum_{j=k+1}^{N K_{v}}\left(\Re\left\{z_{k j} z_{k k}^{-1}\right\}\right)^{2}+4 \sum_{j=1}^{k-1}\left(\Re\left\{z_{k j} z_{k k}^{-1}\right\}\right)^{2} P_{e, j \mid \vec{\alpha}}\right]^{-\frac{1}{2}}\right)
$$

with

$$
P_{e, 1 \mid \vec{\alpha}, \mathrm{SIC}}=Q\left(\left[z_{11}^{-1} \frac{N_{0}}{2 \epsilon_{1}}+\sum_{j=2}^{N K_{v}}\left(\Re\left\{z_{1 j} z_{11}^{-1}\right\}\right)^{2}\right]^{-\frac{1}{2}}\right)
$$


Comparing $P_{e, k \mid \vec{\alpha}, \text { DBSIC }}$ with $P_{e, k \mid \vec{\alpha}, \text { SIC }}$, we note the extra term $\sum_{j=k+1}^{N K_{v}}\left(\Re\left\{z_{k j} z_{k k}^{-1}\right\}\right)^{2}$ in the Q-function of SIC that is caused by a residual MAI. This term dominates the performance of SIC. As a decorrelation is employed at each stage of DBSIC, the residual MAI is eliminated completely, yielding better performance starting from the $1^{s t}$ stage. Hence, the accumulated detection estimate error term at each stage for DBSIC in the Q-function is much less than that for conventional SIC.

The average system performance of DBSIC can be obtained by averaging $P_{e \mid \vec{\alpha}, \text { DBSIC }}$ over the joint pdf of $\vec{\alpha}$. Since no closed-form solution of this integration could be found, an estimate of the average system BER performance of DBSIC can be found by calculating $P_{e \mid \vec{\alpha}, \text { DBSIC }}$ for a sufficiently large number of values of $\vec{\alpha}$ and averaging them. As will be seen later, averaging $P_{e \mid \vec{\alpha}, \text { DBSIC }}$ over at least 100 values of $\vec{\alpha}$ provides a very good estimate of the BER for DBSIC.

The theoretical performance analysis of DBSIC and SIC presented in this section gave some insight why DBSIC performs better than SIC. Recall that the theoretical analysis was derived based on a Gaussian assumption. To demonstrate the validity of this approximation in order to be able later on to predict DBSIC performance without simulating the entire system, simulation results are compared to the corresponding results obtained from the theoretical approximation.

Fig. 5.1 presents the BER averaged over all users versus the SNR per bit $E_{b} / N_{0}$ over a 2-path Rayleigh fading channel for an asynchronous multirate DS-CDMA system employing DBSIC with ten physical users according to the cases outlined in table 5.1. For example, for case 2, there are 7 physical users of rate $R\left(K_{1}=7\right)$ and 3 physical users of rate $2 R$. The processing gain of the lowest rate users is 32 . The results obtained by simulation of the entire system (dashed lines) are obtained as follows: Simulations are run such that at least 15 errors would occur for the lowest BER desired and at least 1000 trials are run. Within each trial each user transmits 5 consecutive 
symbols. Further details on the simulation procedure are given in section 6.1 and section 6.2 including the flow chart presented in Fig. 6.1. The results obtained by using the theoretical analysis (solid lines) are obtained as follows: an estimate of the average system BER performance of DBSIC has been found by calculating $P_{e \mid \vec{\alpha}, \mathrm{DBSIC}}$ for at least 100 values of $\vec{\alpha}$ and averaging them.

Table 5.1 Simulated physical users rates

\begin{tabular}{|r||r|r|r|r|r|}
\hline case & Class1 & Class2 & Class3 & Class4 & virtual users \\
\hline 1 & 10 & 0 & 0 & 0 & tot. \#=10 \\
2 & 7 & 3 & 0 & 0 & tot. \#=13 \\
3 & 5 & 3 & 2 & 0 & tot. \#=17 \\
4 & 4 & 3 & 2 & 1 & tot. \#=20 \\
\hline
\end{tabular}

It is seen from Fig. 5.1 that there is a good match between the simulations (dashed lines) and the curves obtained from the approximate theoretical analysis (solid lines) for single and multi-rate systems. Although some numerical calculations are still required to calculate the system performance using the analytical approach in order to perform the average over the channel fading coefficients, the results can be obtained much more easily than a full scale simulation. Detailed comments on the performance of DBSIC under perfect channel estimation will be given in section 6.4 along with the other simulation results. 


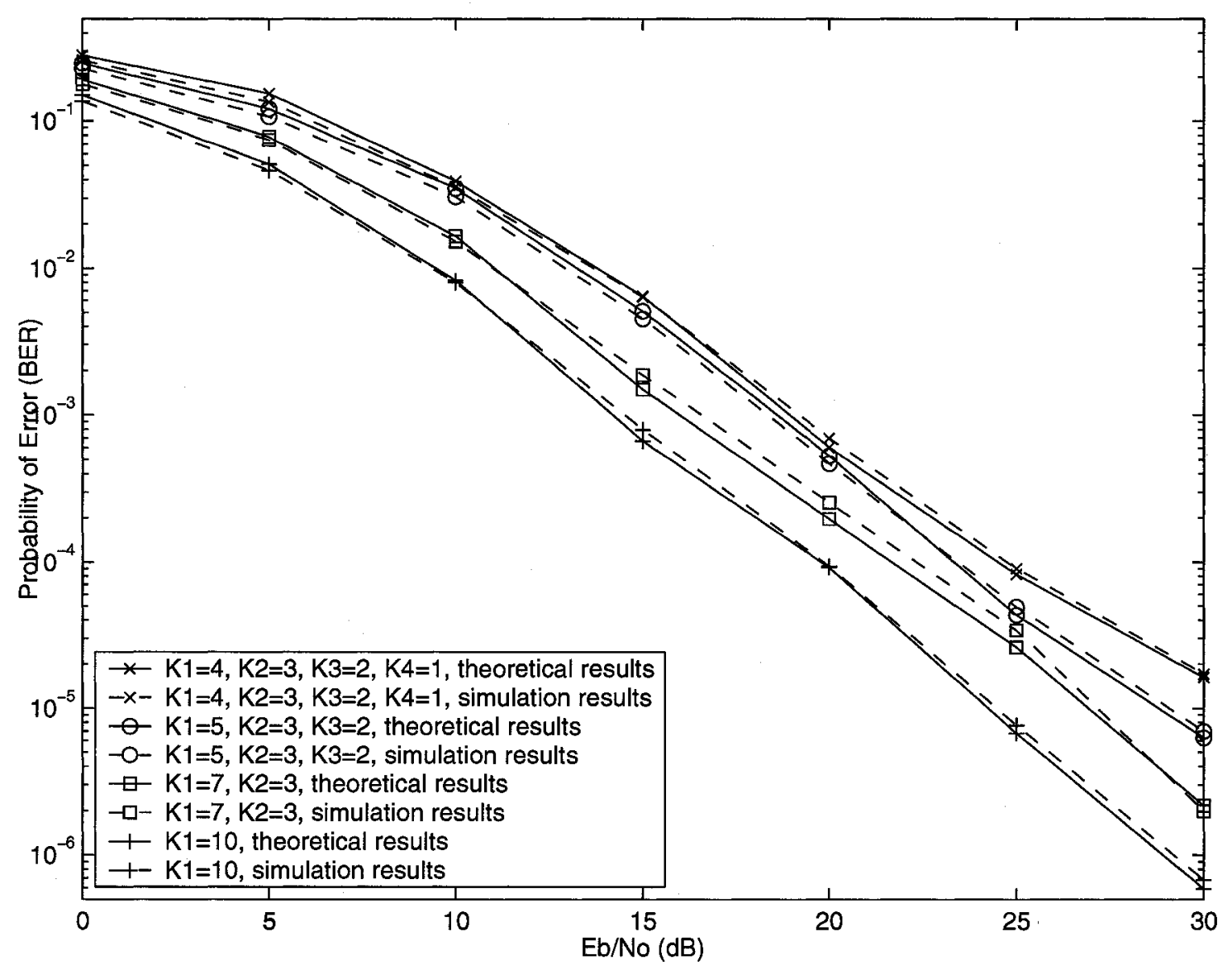

Fig. 5.1 Performance of DBSIC over 2-path Rayleigh fading channel with RAKE, perfect channel estimate, $G_{1}=32$ 


\subsection{AME performance analysis}

\subsubsection{System models and receiver structures for synchronous DS-CDMA systems}

Considering a synchronous single-rate BPSK DS-CDMA system with $K$ physical users, the received signal is

$$
r(t)=\sum_{k=1}^{K}\left\{\sqrt{E_{k}^{s}} b_{k}^{\mathrm{s}} \alpha_{k} S_{k}^{s}(t)\right\}+n(t)
$$

where $b_{k}^{\mathrm{s}} \in\{-1,1\}, E_{k}^{s}, S_{k}^{s}(t)$ and $\alpha_{k}$ are respectively the transmitted bit, signal energy, signature waveform and path gain (modeled as a zero-mean circularly complex Gaussian random variable) of the $k^{t h}$ physical user. The noise $n(t)$ is a zero mean complex Gaussian random process with power spectral density $\frac{N_{0}}{2}$.

Let $C_{k}^{s}(t)=\alpha_{k} S_{k}^{s}(t)$ be the effective signature sequence for the $k^{t h}$ physical user. The received signal is re-written as:

$$
r(t)=\sum_{k=1}^{K} \sqrt{E_{k}^{s}} b_{k}^{s} C_{k}^{s}(t)+n(t)
$$

Assuming that the channel gains are known to the receiver, the sampled outputs of $K$ matched filters are

$$
y_{k}^{\mathrm{s}}=\int_{0}^{T_{s}} r(t) C_{k}^{s *}(t) d t \quad k=1, \ldots, K
$$

where $T_{s}$ is the symbol duration. Let $\vec{y}^{\mathrm{s}}=\left[y_{1}^{\mathrm{s}}, \cdots, y_{K}^{\mathrm{s}}\right]^{T}$. The output vector $\vec{y}^{\mathrm{s}}$ is

$$
\vec{y}^{\mathrm{s}}=\boldsymbol{R}_{\mathrm{s}} \boldsymbol{E}_{\mathrm{s}} \vec{b}^{\mathrm{s}}+\vec{n}^{\mathrm{s}}
$$


where $\boldsymbol{R}_{\mathrm{s}}$ is a correlation matrix of dimension $K \times K$ with $m n^{\text {th }}$ element:

$$
\begin{gathered}
\rho_{n m}^{\mathrm{s}}=\int_{0}^{T_{s}} C_{n}^{s}(t) C_{m}^{s *}(t) d t \\
\boldsymbol{E}_{\mathrm{s}}=\operatorname{diag}\left(\sqrt{E_{1}^{s}}, \cdots, \sqrt{E_{K}^{s}}\right), \vec{b}^{\mathrm{s}}=\left[b_{1}^{\mathrm{s}}, \cdots, b_{K}^{\mathrm{s}}\right]^{T}, \vec{n}^{\mathrm{s}}=\left[n_{1}^{\mathrm{s}}, \cdots, n_{K}^{\mathrm{s}}\right]^{T} \\
n_{k}^{\mathrm{s}}=\int_{0}^{T_{s}} n(t) C_{k}^{s *}(t) d t
\end{gathered}
$$

1. Conventional single-user matched filter (MF) detector:

$$
\hat{\vec{b}}_{\mathrm{MF}}^{\mathrm{s}}=\operatorname{sgn}\left(\Re\left\{\vec{y}^{\mathrm{s}}\right\}\right)
$$

where $\Re\left\{\vec{y}^{\mathrm{s}}\right\}$ represents the vector formed by the real parts of the elements of the vector $\vec{y}^{\mathrm{s}}$.

2. Decorrelating detector:

$$
\hat{\vec{b}}_{\mathrm{DEC}}^{\mathrm{s}}=\operatorname{sgn}\left(\Re\left\{\left[\boldsymbol{R}_{\mathrm{s}} \boldsymbol{E}_{\mathrm{s}}\right]^{-1} \vec{y}^{\mathrm{s}}\right\}\right)
$$

For the conventional SIC and DBSIC, the received signal is passed through the bank of matched filters once and the SIC cancellation/detecting order is determined based on these outputs for all the symbols of the sequence. Let $\vec{y}_{\text {new }}^{\mathrm{s}}$ be the vector obtained by reordering $\vec{y}^{\mathrm{s}}$ so that its elements $y_{i}^{\mathrm{s}, \mathrm{new}}$ are listed in decreasing order. Then

$$
\begin{aligned}
\vec{y}_{\text {new }}^{\mathrm{s}} & =\boldsymbol{R}_{\mathrm{s}, \text { new }} \boldsymbol{E}_{\mathrm{s}, \text { new }} \vec{b}_{\text {new }}^{\mathrm{s}}+\vec{n}_{\text {new }}^{\mathrm{s}}=\mathbf{Z}_{\mathrm{s}} \vec{b}_{\text {new }}^{\mathrm{s}}+\vec{n}_{\text {new }}^{\mathrm{s}} \\
y_{i}^{\mathrm{s}, \text { new }} & =\sum_{j=1}^{K} z_{i j}^{\mathrm{s}} b_{j}^{\mathrm{s} \text {,new }}+n_{i}^{\mathrm{s} \text {,new }} \quad i=1, \cdots, K
\end{aligned}
$$

with $\mathbf{Z}_{\mathrm{s}}=\boldsymbol{R}_{\mathrm{s}, \text { new }} \boldsymbol{E}_{\mathrm{s}, \text { new }}, z_{i j}^{\mathrm{s}}=i j^{\text {th }}$ entry of $\mathbf{Z}_{\mathrm{s}}$ and $b_{j}^{\text {s,new }}=j^{\text {th }}$ element of $\vec{b}_{\text {new }}^{\mathrm{s}}$. 
3. Conventional SIC detector: $\hat{b}_{1, \text { sIC }}^{\text {s,new }}=\operatorname{sgn}\left(\Re\left\{y_{1}^{\mathrm{s}, \text { new }}\right\}\right)$

$$
\hat{b}_{k, \text { sIC }}^{\mathrm{s}, \text { new }}=\operatorname{sgn}\left(\Re\left\{y_{k}^{\mathrm{s}, \text { new }}-\sum_{j=1}^{k-1} z_{k j}^{\mathrm{s}} \hat{b}_{j, \text { sIC }}^{\mathrm{s} \text {,new }}\right\}\right) k=2, \cdots, K
$$

4. DBSIC detector: The first symbol of the strongest user is determined using

$$
\hat{b}_{1, \mathrm{DBSIC}}^{\mathrm{s}, \mathrm{new}}=\operatorname{sgn}\left(\Re\left\{\left[\mathbf{Z}_{\mathrm{s}}^{-1} \vec{y}_{\text {new }}^{\mathrm{s}}\right]_{1}\right\}\right)
$$

but unlike the decorrelating detector that detects all users' signals, only the first signal is determined at this stage. The other $b_{i}^{\text {s,new }}$, s are determined as follows. Define $\vec{y}_{\text {new }}^{\mathrm{s}(k)}=\left[y_{k}^{\mathrm{s}(k)}, \cdots, y_{K}^{\mathrm{s}(k)}\right]^{T}, \vec{n}_{\text {new }}^{\mathrm{s}(k)}=\left[n_{k}^{\mathrm{s}(k)}, \cdots, n_{K}^{\mathrm{s}(k)}\right]^{T}, \vec{b}_{\text {new }}^{\mathrm{s}(k)}=\left[b_{k}^{\mathrm{s}, \text { new }}, \cdots, b_{K}^{\mathrm{s}, \text { new }}\right]^{T}$, where $y_{i}^{\mathrm{s}(1)} \triangleq y_{i}^{\mathrm{s} \text {,new }}$, and for $i=k, \ldots, K, k \geq 2$

$$
\begin{aligned}
y_{i}^{\mathrm{s}(k)} & \triangleq y_{i}^{\mathrm{s}(k-1)}-z_{i k-1}^{\mathrm{s}} \hat{b}_{k-1}^{\mathrm{s}, \text { new }}=\sum_{j=k}^{K} z_{i j}^{\mathrm{s}} b_{j}^{\mathrm{s}, \text { new }}+n_{i}^{\mathrm{s}(k)} \\
n_{i}^{\mathrm{s}(k)} & \triangleq n_{i}^{\mathrm{s}}+\sum_{j=1}^{k-1} z_{i j}^{\mathrm{s}}\left(b_{j}^{\mathrm{s}, \text { new }}-\hat{b}_{j}^{\mathrm{s}, \text { new }}\right) \\
\Longrightarrow \vec{y}_{n e w}^{\mathrm{s}(k)} & =\mathbf{Z}_{\mathrm{s}}^{(k)} \vec{b}_{\text {new }}^{\mathrm{s}(k)}+\vec{n}_{\text {new }}^{\mathrm{s}(k)}
\end{aligned}
$$

where the matrix $\mathbf{Z}_{\mathrm{S}}^{(k)}(K-k+1) \times(K-k+1)$ is given by

$$
\left(\begin{array}{cccc}
z_{k k}^{\mathrm{s}} & z_{k k+1}^{\mathrm{s}} & \cdots & z_{k K}^{\mathrm{s}} \\
z_{k+1 k}^{\mathrm{s}} & z_{k+1 k+1}^{\mathrm{s}} & \cdots & z_{k+1 K}^{\mathrm{s}} \\
\vdots & \vdots & \ddots & \vdots \\
z_{K k}^{\mathrm{s}} & z_{K k+1}^{\mathrm{s}} & \cdots & z_{K K}^{\mathrm{s}}
\end{array}\right)
$$


The estimate of $b_{k}^{\mathrm{s}, \text { new }}$ is obtained using

$$
\hat{b}_{k, \mathrm{DBSTC}}^{\mathrm{s}, \text { new }}=\operatorname{sgn}\left(\Re\left\{\left[\left\{\mathbf{Z}_{\mathrm{s}}^{(k)}\right\}^{-1} \vec{y}_{\text {new }}^{\mathrm{s}(k)}\right]_{1}\right\}\right)
$$

\subsubsection{AME performance analysis over AWGN channels}

AME is another performance analysis tool that is often used to evaluate the performance of wireless CDMA systems, especially in the analysis of multiuser detectors. AME is referred to as the asymptotic limit of the loss in the SNR as the noise power spectral density approaches zero. Let $P_{e, k}(\sigma)$ be the BER of the $k^{\text {th }}$ user and let $E_{k}^{s}$ be the actual energy of the $k^{\text {th }}$ user. Let us define the effective energy of the $k^{\text {th }}$ user as the energy required by user $k$ such that it achieves the same $P_{e, k}(\sigma)$ in the same AWGN channel but without the MAI, i.e. $e_{k}(\sigma)$ is defined such that $P_{e, k}(\sigma)=Q\left(\sqrt{\frac{e_{k}(\sigma)}{\sigma^{2}}}\right)$, where $\sigma^{2}=N_{0} / 2$ is the power spectral density of the additive white Gaussian noise. Then AME $\eta$ is defined as [2]

$$
\eta_{k}=\lim _{\sigma \rightarrow 0} \frac{e_{k}(\sigma)}{E_{k}^{s}}
$$

or can also be defined as

$$
\begin{aligned}
\eta_{k} & =\sup _{r \in[0,1]}\left\{\lim _{\sigma \rightarrow 0} \frac{P_{e, k}(\sigma)}{Q\left(\sqrt{\frac{r E_{k}^{s}}{\sigma^{2}}}\right)}=0\right\} \\
& =\frac{2}{E_{k}^{s}} \lim _{\sigma \rightarrow 0} \sigma^{2} \log \left[\frac{1}{P_{e, k}(\sigma)}\right]
\end{aligned}
$$

Two-user synchronous (or one-shot asynchronous) systems over AWGN and Rayleigh fading channels are considered for the AME performance evaluation. For convenience, the notation $\sigma^{2}=\frac{N_{0}}{2}$ is used in the following derivations.

For the AWGN channel with 2 users, the matched filter outputs (5.3) are simplified 
as:

$$
\begin{gathered}
\vec{y}^{\mathrm{s}}=\left(\begin{array}{c}
y_{1}^{\mathrm{s}} \\
y_{2}^{\mathrm{s}}
\end{array}\right)=\boldsymbol{R}_{\mathrm{s}} \boldsymbol{E}_{\mathrm{s}} \vec{b}^{\mathrm{s}}+\vec{n}^{\mathrm{s}}=\left(\begin{array}{cc}
1 & \rho \\
\rho & 1
\end{array}\right)\left(\begin{array}{cc}
\sqrt{E_{1}^{s}} & 0 \\
0 & \sqrt{E_{2}^{s}}
\end{array}\right)\left(\begin{array}{c}
b_{1}^{\mathrm{s}} \\
b_{2}^{\mathrm{s}}
\end{array}\right)+\left(\begin{array}{c}
n_{1}^{\mathrm{s}} \\
n_{2}^{\mathrm{s}}
\end{array}\right) \\
\left\{\begin{array}{l}
y_{1}^{\mathrm{s}}=\sqrt{E_{1}^{s}} b_{1}^{\mathrm{s}}+\sqrt{E_{2}^{s}} \rho b_{2}^{\mathrm{s}}+n_{1}^{\mathrm{s}} \\
y_{2}^{\mathrm{s}}=\sqrt{E_{1}^{s}} \rho b_{1}^{\mathrm{s}}+\sqrt{E_{2}^{s}} b_{2}+n_{2}^{\mathrm{s}}
\end{array}\right.
\end{gathered}
$$

where

$$
\rho=\int_{0}^{T_{s}} S_{1}(t) S_{2}^{*}(t) d t
$$

is the cross-correlation between user 1 and user 2 , and $\vec{n}^{\mathrm{s}}=\left(n_{1}^{\mathrm{s}}, n_{2}^{\mathrm{s}}\right)^{T}$ has zero mean and autocorrelation function $E\left[\vec{n}^{\mathrm{s}} \vec{n}^{\mathrm{s}} / 2\right]=\left(N_{0} / 2\right) \boldsymbol{R}_{\mathrm{s}}$.

Referring to [2], the AME for the conventional single-user MF, optimum, decorrelating and SIC receivers are given as follows:

1. Conventional MF receiver [2]:

$$
\begin{aligned}
& \eta_{1}=\left(\max \left\{0,1-\sqrt{\frac{E_{2}^{s}}{E_{1}^{s}}}|\rho|\right\}\right)^{2} \\
& \eta_{2}=\left(\max \left\{0,1-\sqrt{\frac{E_{1}^{s}}{E_{2}^{s}}}|\rho|\right\}\right)^{2}
\end{aligned}
$$

2. Optimum receiver [2]:

$$
\begin{aligned}
& \eta_{1}=\min \left\{1,1+\frac{E_{2}^{s}}{E_{1}^{s}}-2 \sqrt{\frac{E_{2}^{s}}{E_{1}^{s}}}|\rho|\right\} \\
& \eta_{2}=\min \left\{1,1+\frac{E_{1}^{s}}{E_{2}^{s}}-2 \sqrt{\frac{E_{1}^{s}}{E_{2}^{s}}}|\rho|\right\}
\end{aligned}
$$


3. Decorrelating receiver [2]:

$$
\eta_{1}=\eta_{2}=1-\rho^{2}
$$

4. SIC receiver [2]:

$$
\eta_{2}= \begin{cases}\eta_{1}=\left(\max \left\{0,1-\sqrt{\frac{E_{2}^{s}}{E_{1}^{s}}}|\rho|\right\}\right)^{2} \\
\left\{\begin{array}{ll}
1-4 \sqrt{\frac{E_{1}^{s}}{E_{2}^{s}}}|\rho|+\frac{1}{1-\rho^{2}} \frac{E_{1}^{s}}{E_{2}^{s}}, & \sqrt{\frac{E_{1}^{s}}{E_{2}^{s}}} \leq \frac{1}{|\rho|}-|\rho| \\
\left(\sqrt{\frac{E_{1}^{s}}{E_{2}^{s}}}-|\rho|\right)^{2}, & \frac{1}{|\rho|}-|\rho| \leq \sqrt{\frac{E_{1}^{s}}{E_{2}^{s}}} \leq 1+|\rho| \\
1, & 1+|\rho|<\sqrt{\frac{E_{1}^{s}}{E_{2}^{s}}}
\end{array},|\rho| \geq \frac{1}{2}\right. \\
\left\{\begin{array}{ll}
1-4 \sqrt{\frac{E_{s}^{s}}{E_{2}^{s}}}|\rho|+\frac{1}{1-\rho^{2}} \frac{E_{1}^{s}}{E_{2}^{s}}, & \sqrt{\frac{E_{s}^{s}}{E_{2}^{s}}} \leq 4|\rho|\left(1-\rho^{2}\right) \\
1, & \sqrt{\frac{E_{1}^{s}}{E_{2}^{s}}}>4|\rho|\left(1-\rho^{2}\right)
\end{array},|\rho|<\frac{1}{2}\right.\end{cases}
$$

\section{AME of DBSIC for AWGN channels}

For simplicity we assume that user 1 is the first user to be canceled (no reordering done, $\vec{y}_{\text {new }}^{\mathrm{s}}=\vec{y}^{\mathrm{s}}, \vec{b}_{\text {new }}^{\mathrm{s}}=\vec{b}^{\mathrm{s}}$ ). Since the estimate of the first bit using DBSIC is the same as the one that would be obtained with the decorrelating detector, we have

$$
\eta_{1}=\eta_{1, \mathrm{dec}}=1-\rho^{2}
$$

The $\eta_{2}$ can be obtained as (see Appendix D) [133]

$$
\eta_{2}=\min \left\{1, \frac{E_{1}^{s}}{E_{2}^{s}}\left(1-\rho^{2}\right)+\left(\max \left\{0,1-2|\rho| \sqrt{\frac{E_{1}^{s}}{E_{2}^{s}}}\right\}\right)^{2}\right\}
$$




\section{AME performance comparison over AWGN channels}

Fig. 5.2 shows the AME results for DBSIC, optimum, conventional SIC, and decorrelating receivers over the AWGN channel when $\rho=0.5$ corresponding to an intermediate correlation value between the users spreading sequences. It is observed that DBSIC receiver has a lower AME than the optimum receiver when the interfering user is weaker than the user of interest and achieves the unity optimum receiver AME as the interferer becomes stronger than the user of interest. The decorrelating receiver has a constant AME due to the fact its performance does not depend on the received energy of all users, i.e. it is completely near-far resistant. When the interferer is much weaker or slightly stronger than the user of interest the DBSIC AME is better than that of the decorrelating detector but is worse when the interferer is not much weaker. It is also observed that DBSIC shows a similar AME curve shape as the conventional SIC receiver but with higher AME performance for all user energy ratios. Such trend is also seen for other values of the spreading codes correlation as illustrated in Fig. 5.3 (highly correlated scenario, $\rho=0.8$ ) and Fig. 5.4 (low correlated scenario, $\rho=0.2$ ). Combining both the decorrelating and SIC schemes, DBSIC implements a decorrelating process at each stage of the conventional SIC so that smaller decision errors and hence less error propagation from one stage to the next are obtained yielding improved performance. In a highly correlated scenario such as in Fig. 5.3 where $\rho$ equals to 0.8 , the AME performance of all receivers degrades and the inverse bell shape for optimum, DBSIC and SIC becomes deeper as the minimum shifts lower. In a low correlated scenario where $\rho$ is equal to 0.2 , illustrated in Fig. 5.4, all detectors except MF have a significant AME performance improvement and approach the unity optimum receiver AME as expected. 


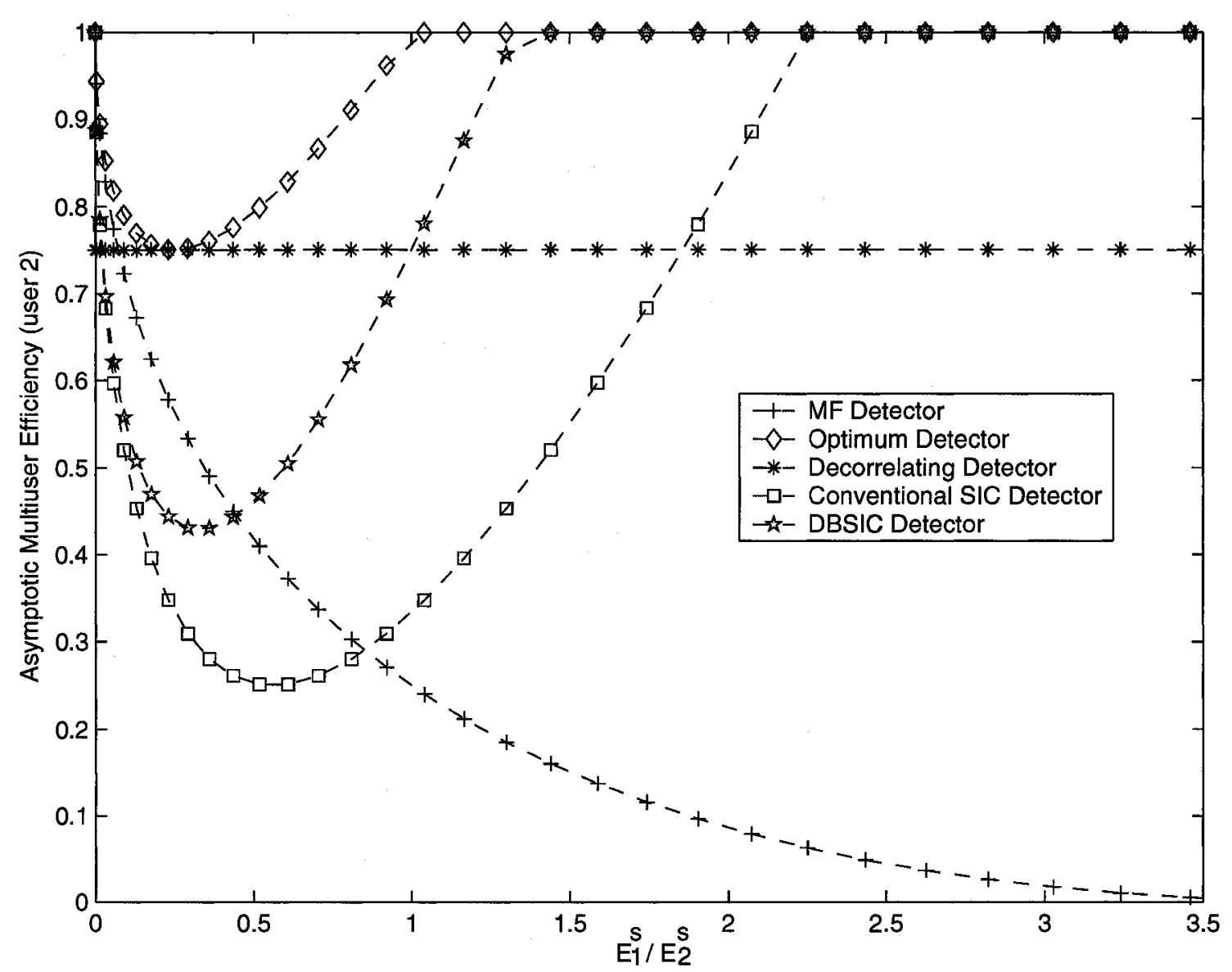

Fig. 5.2 Asymptotic Multiuser Efficiency for user 2 over the AWGN Channel, $\rho=0.5$

\subsubsection{AME performance analysis over a Rayleigh flat fading channel}

The definition of AME in an Rayleigh fading channel is different from the AWGN one and is given as

$$
\eta_{k}=\lim _{\sigma \rightarrow 0} \frac{\sigma^{2}}{4 E_{k}^{s} P_{e, k}(\sigma)}
$$




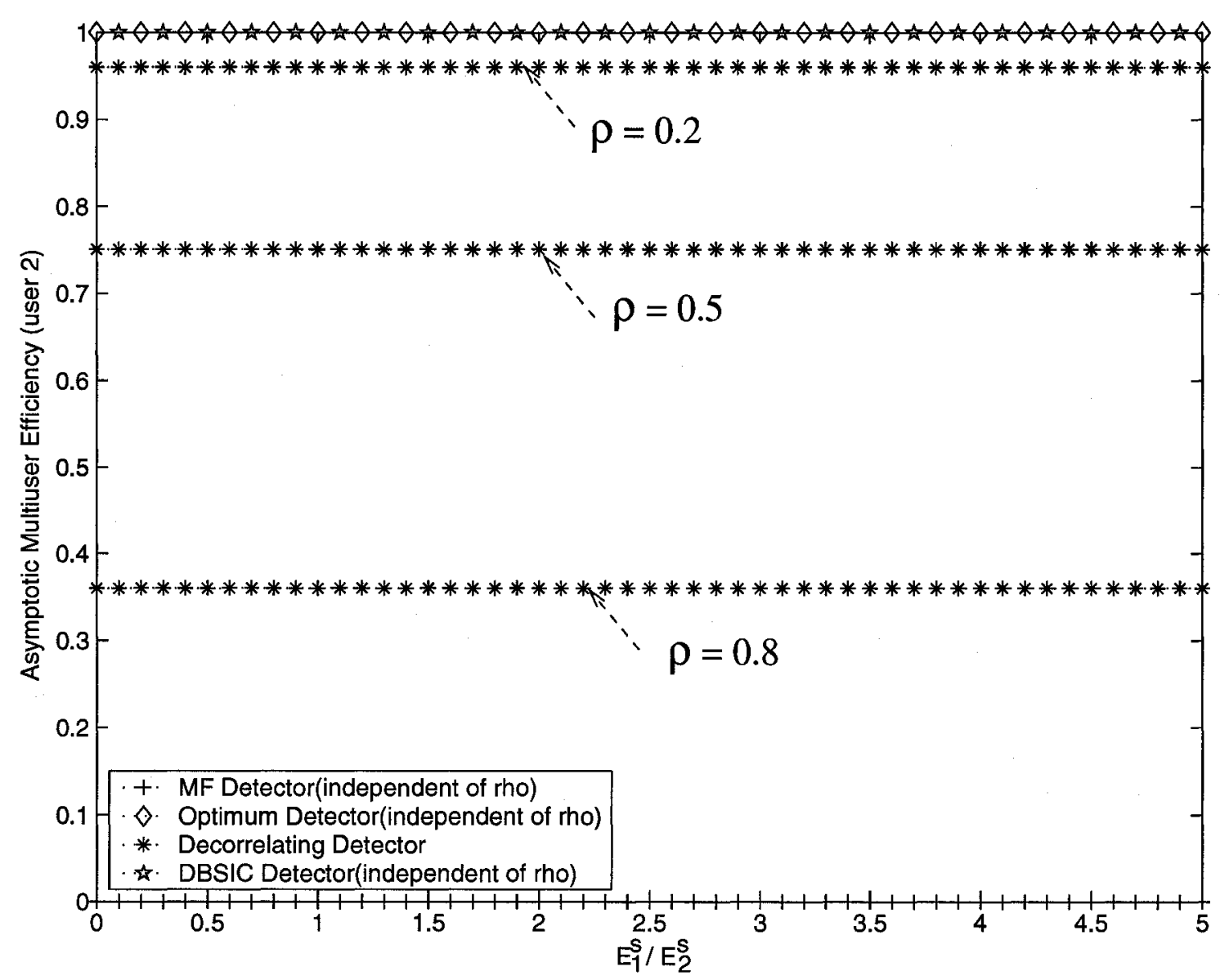

Fig. 5.3 Asymptotic Multiuser Efficiency for user 2 over the AWGN Channel, $\rho=0.8$

Considering a two-user system over a single-path Rayleigh fading channel, the matched filter outputs (5.3) reduces to

$$
\vec{y}^{\mathrm{s}}=\left(\begin{array}{c}
y_{1}^{\mathrm{s}} \\
y_{2}^{\mathrm{s}}
\end{array}\right)=\boldsymbol{R}_{\mathrm{s}} \boldsymbol{E}_{\mathrm{s}} \vec{b}^{\mathrm{s}}+\vec{n}^{\mathrm{s}}=\left(\begin{array}{cc}
\rho_{11}^{\mathrm{s}} & \rho_{21}^{\mathrm{s}} \\
\rho_{12}^{\mathrm{s}} & \rho_{22}^{\mathrm{s}}
\end{array}\right)\left(\begin{array}{cc}
\sqrt{E_{1}^{s}} & 0 \\
0 & \sqrt{E_{2}^{s}}
\end{array}\right)\left(\begin{array}{c}
b_{1}^{\mathrm{s}} \\
b_{2}^{\mathrm{s}}
\end{array}\right)+\left(\begin{array}{c}
n_{1}^{\mathrm{s}} \\
n_{2}^{\mathrm{s}}
\end{array}\right)
$$

where

$$
\rho_{11}^{\mathrm{s}}=\int_{0}^{T_{s}} C_{1}(t) C_{1}^{*}(t) d t=\int_{0}^{T_{s}} \alpha_{1} S_{1}(t) \alpha_{1}^{*} S_{1}^{*}(t) d t=\left|\alpha_{1}\right|^{2}
$$




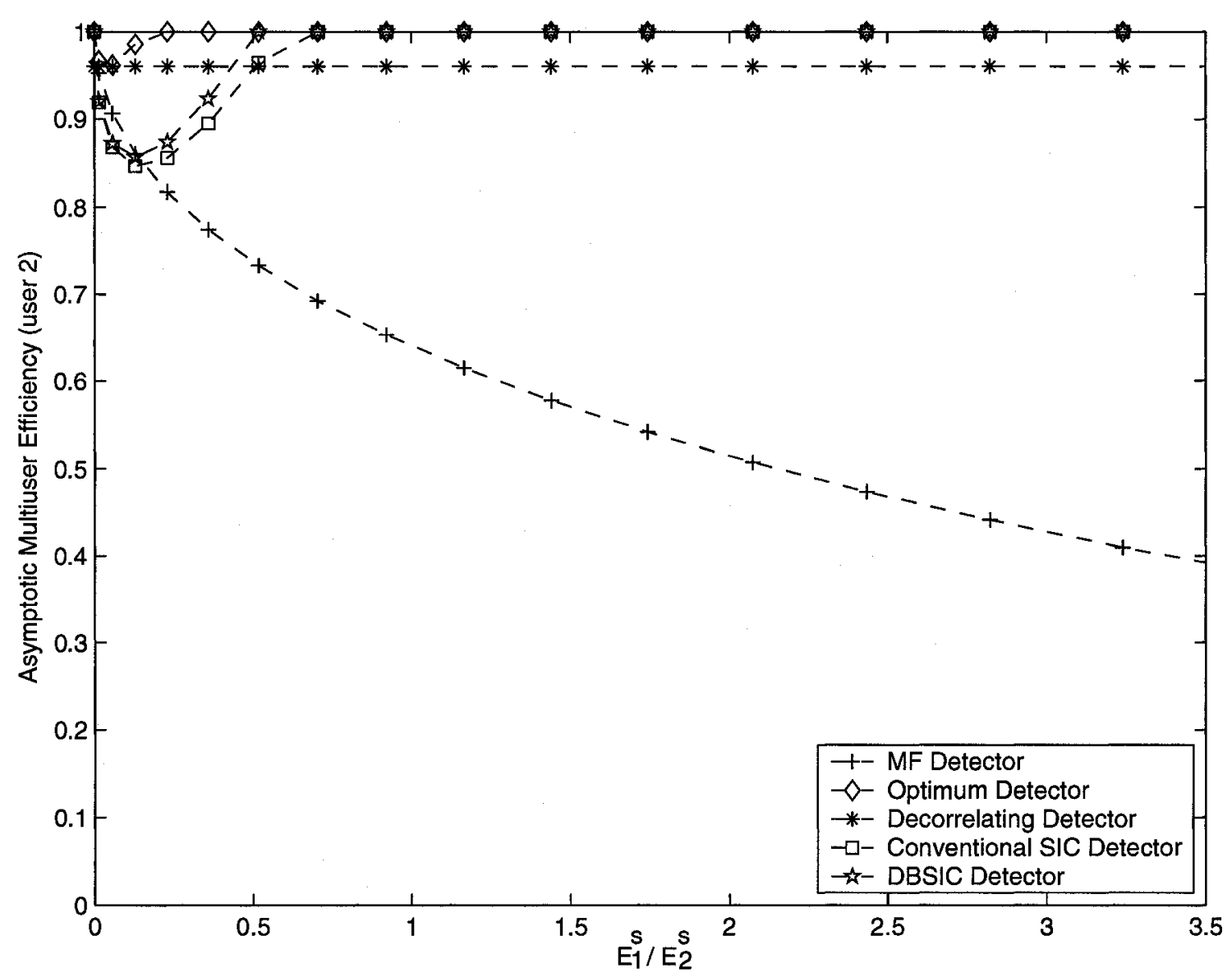

Fig. 5.4 Asymptotic Multiuser Efficiency for user 2 over the AWGN Channel, $\rho=0.2$

$$
\begin{aligned}
& \rho_{12}^{\mathrm{s}}=\int_{0}^{T_{s}} C_{1}(t) C_{2}^{*}(t) d t=\int_{0}^{T_{s}} \alpha_{1} S_{1}(t) \alpha_{2}^{*} S_{2}^{*}(t) d t=\alpha_{1} \alpha_{2}^{*} \rho \\
& \rho_{21}^{\mathrm{s}}=\int_{0}^{T_{s}} C_{2}(t) C_{1}^{*}(t) d t=\int_{0}^{T_{s}} \alpha_{2} S_{2}(t) \alpha_{1}^{*} S_{1}^{*}(t) d t=\alpha_{1}^{*} \alpha_{2} \rho \\
& \rho_{22}^{\mathrm{s}}=\int_{0}^{T_{s}} C_{2}(t) C_{2}^{*}(t) d t=\int_{0}^{T_{s}} \alpha_{2} S_{2}(t) \alpha_{2}^{*} S_{2}^{*}(t) d t=\left|\alpha_{2}\right|^{2}
\end{aligned}
$$

and where $\rho$ is the cross-correlation between user 1 and user 2 given by (5.9).

$$
\left\{\begin{array}{l}
y_{1}^{\mathrm{s}}=\sqrt{E_{1}^{s}} \rho_{11}^{\mathrm{s}} b_{1}^{\mathrm{s}}+\sqrt{E_{2}^{s}} \rho_{21}^{\mathrm{s}} b_{2}^{\mathrm{s}}+n_{1}^{\mathrm{s}}=\left|\alpha_{1}\right|^{2} \sqrt{E_{1}^{s}} b_{1}^{\mathrm{s}}+\alpha_{2} \alpha_{1}^{*} \sqrt{E_{2}^{\mathrm{s}}} \rho b_{2}^{\mathrm{s}}+n_{1}^{\mathrm{s}} \\
y_{2}^{\mathrm{s}}=\sqrt{E_{1}^{s}} \rho_{12}^{\mathrm{s}} b_{1}^{\mathrm{s}}+\sqrt{E_{2}^{\mathrm{s}}} \rho_{22}^{\mathrm{s}} b_{2}^{\mathrm{s}}+n_{2}^{\mathrm{s}}=\alpha_{1} \alpha_{2}^{*} \sqrt{E_{1}^{s}} \rho b_{1}^{\mathrm{s}}+\left|\alpha_{2}\right|^{2} \sqrt{E_{2}^{s}} b_{2}^{\mathrm{s}}+n_{2}^{\mathrm{s}}
\end{array}\right.
$$


For the Rayleigh fading channel, $\left|\alpha_{k}\right|$ has a Rayleigh probability distribution:

$$
P_{R}(r)=r e^{-r^{2} / 2}, \quad r \geq 0
$$

1. Conventional MF receiver [2]:

$$
\eta_{1}=\eta_{2}=0
$$

2. Optimum receiver [2]:

$$
\eta_{1}=\eta_{2}=1
$$

3. Decorrelating receiver [2]:

$$
\eta_{1}=\eta_{2}=1-\rho^{2}
$$

\section{AME of DBSIC for Rayleigh Channels}

$$
\begin{aligned}
& P_{e, 1 \mid \alpha_{1}}\left(N_{0}\right)=P\left(\hat{b}_{1}^{\mathrm{s}} \neq b_{1}^{\mathrm{s}} \mid \alpha_{1}\right) \\
& =P\left(\hat{b}_{1}^{\mathrm{s}}=-1 \mid b_{1}^{\mathrm{s}}=1, \alpha_{1}\right) \cdot P\left(b_{1}^{\mathrm{s}}=1 \mid \alpha_{1}\right)+P\left(\hat{b}_{1}^{\mathrm{s}}=1 \mid b_{1}^{\mathrm{s}}=-1, \alpha_{1}\right) \cdot P\left(b_{1}^{\mathrm{s}}=-1 \mid \alpha_{1}\right) \\
& =\frac{1}{2} P\left(\hat{b}_{1}^{\mathrm{s}}=-1 \mid b_{1}^{\mathrm{s}}=1, \alpha_{1}\right)+\frac{1}{2} P\left(\hat{b}_{1}^{\mathrm{s}}=1 \mid b_{1}^{\mathrm{s}}=-1, \alpha_{1}\right) \\
& =P\left(\hat{b}_{1}^{\mathrm{s}}=-1 \mid b_{1}^{\mathrm{s}}=1, \alpha_{1}\right) \text { or }=P\left(\hat{b}_{1}^{\mathrm{s}}=-1 \mid \hat{b}_{1}^{\mathrm{s}}=1, \alpha_{1}\right) \quad \text { (due to symmetry) }
\end{aligned}
$$

It is obtained that (see Appendix E) [133]

$$
P_{e, 1}\left(N_{0}\right)=\frac{1}{2}\left(1-\frac{1}{\sqrt{1+\frac{N_{0} / 2}{E_{1}^{s}\left(1-\rho^{2}\right)}}}\right)
$$


and

$$
\eta_{1}=1-\rho^{2}
$$

For DBSIC,

$$
\begin{gathered}
y_{2}^{\mathrm{s}(2)}=y_{2}^{\mathrm{s}(1)}-z_{21}^{\mathrm{s}} \hat{b}_{1}^{\mathrm{s}}=y_{2}^{\mathrm{s}(1)}-\left[\mathbf{Z}_{\mathrm{s}}\right]_{21} \hat{b}_{1}^{\mathrm{s}}=y_{2}^{\mathrm{s}(1)}-\left[\boldsymbol{R}_{\mathrm{s}} \boldsymbol{E}_{\mathrm{s}}\right]_{21} \hat{b}_{1}^{\mathrm{s}}=y_{2}^{\mathrm{s}(1)}-\rho_{12}^{\mathrm{s}} \sqrt{E_{1}^{\mathrm{s}}} \hat{b}_{1}^{\mathrm{s}} \\
=y_{2}^{\mathrm{s}(1)}-\alpha_{1} \alpha_{2}^{*} \rho \sqrt{E_{1}^{\mathrm{s}}} \hat{b}_{1}^{\mathrm{s}}=y_{2}^{\mathrm{s}}-\alpha_{1} \alpha_{2}^{*} \rho \sqrt{E_{1}^{\mathrm{s}}} \hat{b}_{1}^{\mathrm{s}} \\
=\alpha_{1} \alpha_{2}^{*} \sqrt{E_{1}^{s}} \rho b_{1}^{\mathrm{s}}+\left|\alpha_{2}\right|^{2} \sqrt{E_{2}^{\mathrm{s}}} b_{2}^{\mathrm{s}}+n_{2}^{\mathrm{s}}-\alpha_{1} \alpha_{2}^{*} \rho \sqrt{E_{1}^{\mathrm{s}}} \hat{b}_{1}^{\mathrm{s}} \quad \text { using }(5.20) \\
=\left|\alpha_{2}\right|^{2} \sqrt{E_{2}^{\mathrm{s}}} b_{2}^{\mathrm{s}}+n_{2}^{\mathrm{s}}+\alpha_{1} \alpha_{2}^{*} \sqrt{E_{1}^{\mathrm{s}}} \rho\left(b_{1}^{\mathrm{s}}-\hat{b}_{1}^{\mathrm{s}}\right) \\
\hat{b}_{2}^{\mathrm{s}}=\operatorname{sgn}\left(\Re\left\{\left[\left(\mathbf{Z}_{\mathrm{s}}^{(2)}\right)^{-1} \vec{y}^{\mathrm{s}(2)}\right]_{1}\right\}\right) \\
=\operatorname{sgn}\left(\Re\left\{b_{2}^{\mathrm{s}}+\frac{1}{\left|\alpha_{2}\right|^{2} \sqrt{E_{2}^{s}}} n_{2}^{\mathrm{s}}+\frac{\alpha_{1}}{\alpha_{2}} \sqrt{\frac{E_{1}^{s}}{E_{2}^{s}}} \rho\left(b_{1}^{\mathrm{s}}-\hat{b}_{1}^{\mathrm{s}}\right)\right\}\right)
\end{gathered}
$$

since $z_{21}^{\mathbf{s}}=\alpha_{1} \alpha_{2}^{*} \sqrt{E_{1}^{s}} \rho$ and $\mathbf{Z}_{\mathbf{s}}^{(2)}=\left[z_{22}^{\mathbf{s}}\right]=\left[\rho_{22}^{\mathbf{s}} \sqrt{E_{2}^{s}}\right]=\left[\left|\alpha_{2}\right|^{2} \sqrt{E_{2}^{s}}\right]$. Assuming equally likely transmitted bits, the BER of user 2 is

$$
\begin{aligned}
& P_{e, 2 \mid \alpha_{1}, \alpha_{2}}\left(N_{0}\right)=P\left(\hat{b}_{2}^{\mathrm{s}} \neq b_{2}^{\mathrm{s}} \mid \alpha_{1}, \alpha_{2}\right) \\
& =P\left(\hat{b}_{2}^{\mathrm{s}}=1 \mid b_{2}^{\mathrm{s}}=-1, \alpha_{1}, \alpha_{2}\right) \text { or }=P\left(\hat{b}_{2}^{\mathrm{s}}=-1 \mid b_{2}^{\mathrm{s}}=1, \alpha_{1}, \alpha_{2}\right) \quad \text { (due to symmetry) } \\
& =P\left(\hat{b}_{2}^{\mathrm{s}}=1 \mid b_{2}^{\mathrm{s}}=-1, \alpha_{1}, \alpha_{2}\right) \\
& =P\left(\hat{b}_{2}^{\mathrm{s}}=1 \mid\left(b_{2}^{\mathrm{s}}=-1, b_{1}^{\mathrm{s}}=1\right), \alpha_{1}, \alpha_{2}\right) \cdot P\left(b_{1}^{\mathrm{s}}=1 \mid \alpha_{1}, \alpha_{2}\right) \\
& \quad+P\left(\hat{b}_{2}^{\mathrm{s}}=1 \mid\left(b_{2}^{\mathrm{s}}=-1, b_{1}^{\mathrm{s}}=-1\right), \alpha_{1}, \alpha_{2}\right) \cdot P\left(b_{1}^{\mathrm{s}}=-1 \mid \alpha_{1}, \alpha_{2}\right) \\
& =\frac{1}{2} P\left(\hat{b}_{2}^{\mathrm{s}}=1 \mid\left(b_{2}^{\mathrm{s}}=-1, b_{1}^{\mathrm{s}}=1\right), \alpha_{1}, \alpha_{2}\right)+\frac{1}{2} P\left(\hat{b}_{2}^{\mathrm{s}}=1 \mid\left(b_{2}^{\mathrm{s}}=-1, b_{1}^{\mathrm{s}}=-1\right), \alpha_{1}, \alpha_{2}\right)
\end{aligned}
$$


Starting from this expression it can be obtained that (see Appendix E)

$$
\eta_{2}=\lim _{N_{0} \rightarrow 0} \frac{N_{0}}{8 E_{2}^{s} P_{e, 2}\left(N_{0}\right)}=1
$$

\section{AME performance comparison over Rayleigh channels}

The AME of user 1 for the Rayleigh fading channel case is presented in Fig. 5.5 for various values of $\rho$. It is seen that both DBSIC and the decorrelating detectors have the same AME, which is lower than the optimum scheme one. When $\rho$ becomes smaller, their AME performance approaches close to that of the optimum detector which is unity. Fig. 5.6 illustrates user $2 \mathrm{AME}$ and shows that DBSIC and the optimum detector have the same AME value, which is independent of the value of $\rho$, and have higher AME than the decorrelating detector. Finally it is seen that DBSIC is completely near-far resistant for both users, since its AME remains positive for users energy ratios. 


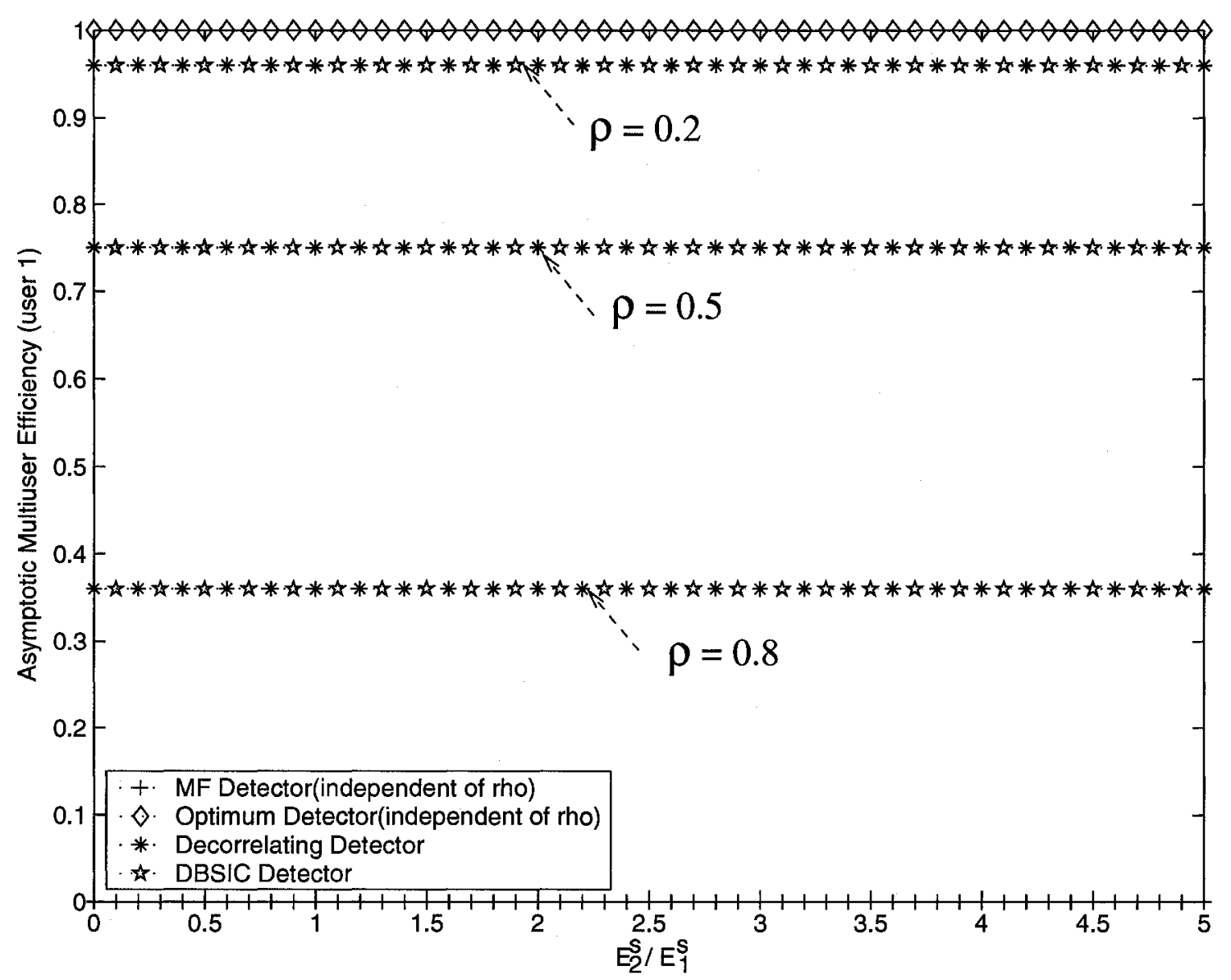

Fig. 5.5 Asymptotic Multiuser Efficiency for user 1 over a Rayleigh Fading Channel 


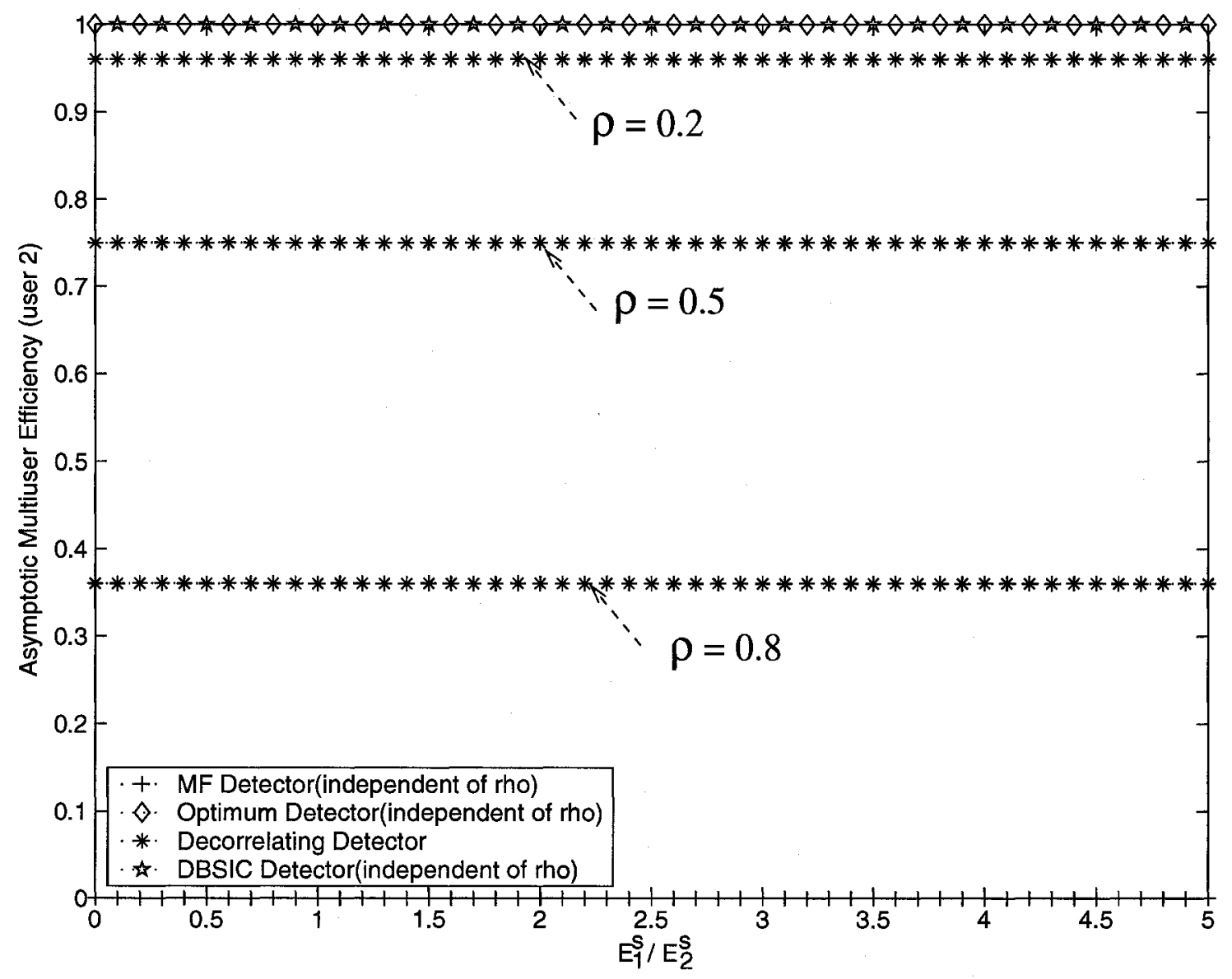

Fig. 5.6 Asymptotic Multiuser Efficiency for user 2 over a Rayleigh Fading Channel 


\section{Chapter 6}

\section{Simulation Results and Discussions}

\subsection{Assumptions and specifications}

The following assumptions are used in this work:

- Uplink simulation of CDMA only;

- No filtering;

- No error control coding;

- Noise is AWGN;

- Small scale fading considered only (in other words, perfect power control is assumed to compensate for large scale fading effects)

- The number of fingers employed in the RAKE receiver is equal to the number of channel paths $L$;

- Only one antenna employed at the receiver;

- The number of the active users $K$ in the system is smaller than the spreading gain. 
An asynchronous multirate DS-CDMA system is considered in all simulations. The system is assumed to be symbol-asynchronous but perfectly chip-synchronous. This means that transmission delays of users are integer multiples of chip intervals. We assume that all virtual users of all classes are such that $E_{c, k}^{(j)}=1$ for $c=1, \ldots, C, k=$ $1, \ldots, K_{c}, j=1, \ldots, c$. The code sequences assigned to each virtual user are kept constant for the whole simulation set once generated. Recall from section 3.1 there are two ways of implementing a VPG DS-CDMA system or equivalently two ways of choosing the spreading sequences of the virtual users corresponding to one high rate physical user. Unless otherwise stated, the second method will be used in the simulations using Gold codes as baseline. Since Gold sequences yield odd processing gains, to obtain even processing gains, one random chip is added to the Gold sequences [80]. A two-path equal gain Rayleigh slow fading channel model is considered. The fading gains of all physical users are assumed to be mutually independent, constant over a sequence of 5 consecutive bits (BPSK modulation) and are implemented based on Clarke's model [130]. It is also assumed that the receiver for each user has perfect knowledge of the spreading codes and delays of all users in the system. In the case of perfect channel estimation, it is also assumed that each user has perfect knowledge of the fading gains of all users. Hard bit decisions are used in the simulations with no coding.

BER performance of the proposed DBSIC scheme and various commonly used CDMA detectors such as the conventional MF detector, decorrelating detector, MMSE detector, SIC detector (with cancellation in the order of received signals' energy or random order), PIC detector, decorrelating DF detector are simulated and compared. To evaluate the exact system BERs and/or to determine the accuracy of analytical approximations, Monte Carlo simulations must be used. A widely used approximation for estimating the accuracy of Monte Carlo simulations is that for a large number of 
trials ten independent errors will result in a $95 \%$ confidence interval. In this study, we run simulations such that at least 15 errors would occur for the lowest BER desired and at least 1000 trials are run. The BER averaged over all users is plotted against the SNR per bit $E_{b} / N_{0}$, where each point is averaged over at least 1000 trials depending on the value of BER obtained, and within each trial each user transmits 5 consecutive bits.

\subsection{Flowchart}

Fig. 6.1 shows the flowchart for the simulation code with no channel estimation scheme implemented. The simulation considers either the receiver knows perfectly the channel information (perfect channel estimation scenario) or the receiver has only access to estimated channel information which differs from the true channel information (imperfect channel estimation scenario).

Fig. 6.2 shows the flowchart for the simulation code with the channel estimation scheme implemented. In this case, the channel information is obtained through the channel estimation scheme where a series of training symbols are generated and fed into the channel estimator. With the estimated channel information, the multiuser detector starts to detect the transmitted symbols for each user in the system. The detected symbols can then be fed back to the channel estimation scheme as the new training symbols to improve the channel estimation performance. This process can be repeated for couple of times but basically one iteration will be carried out to ease the computational time. 


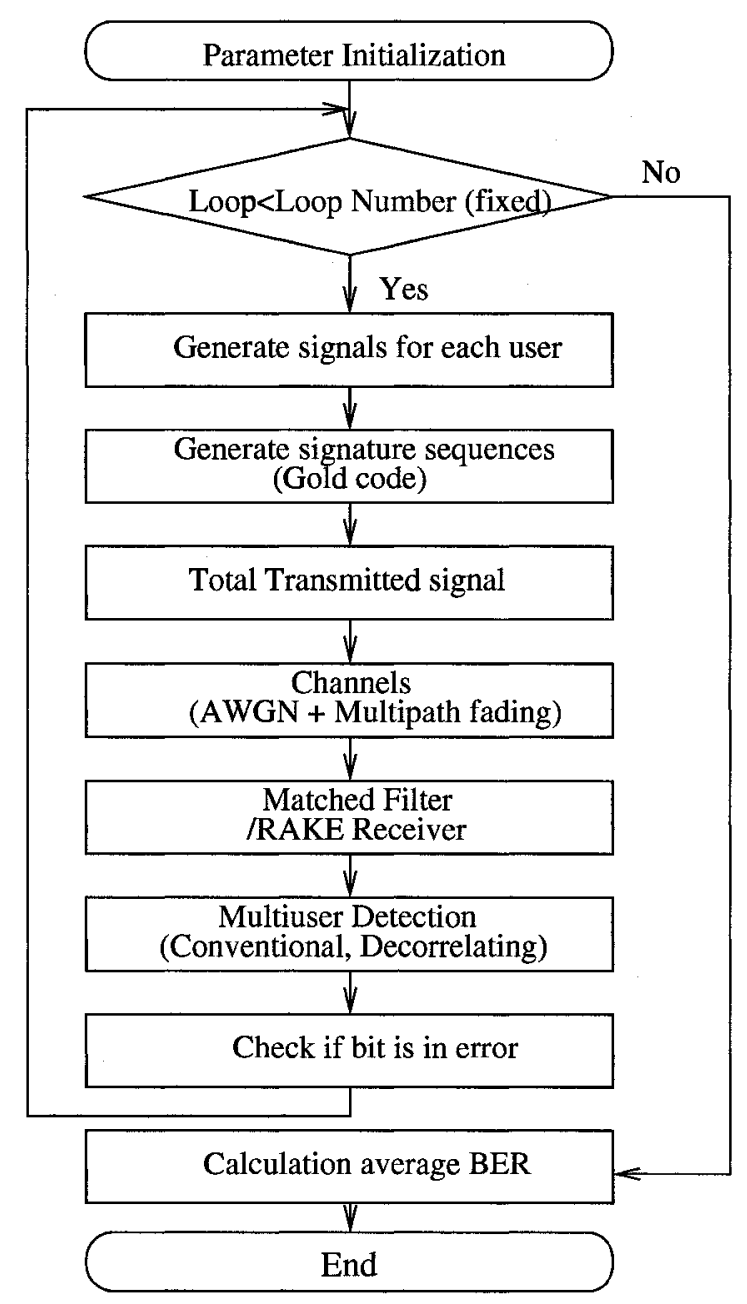

Fig. 6.1 Flowchart for the simulation code with no channel estimation scheme implemented 


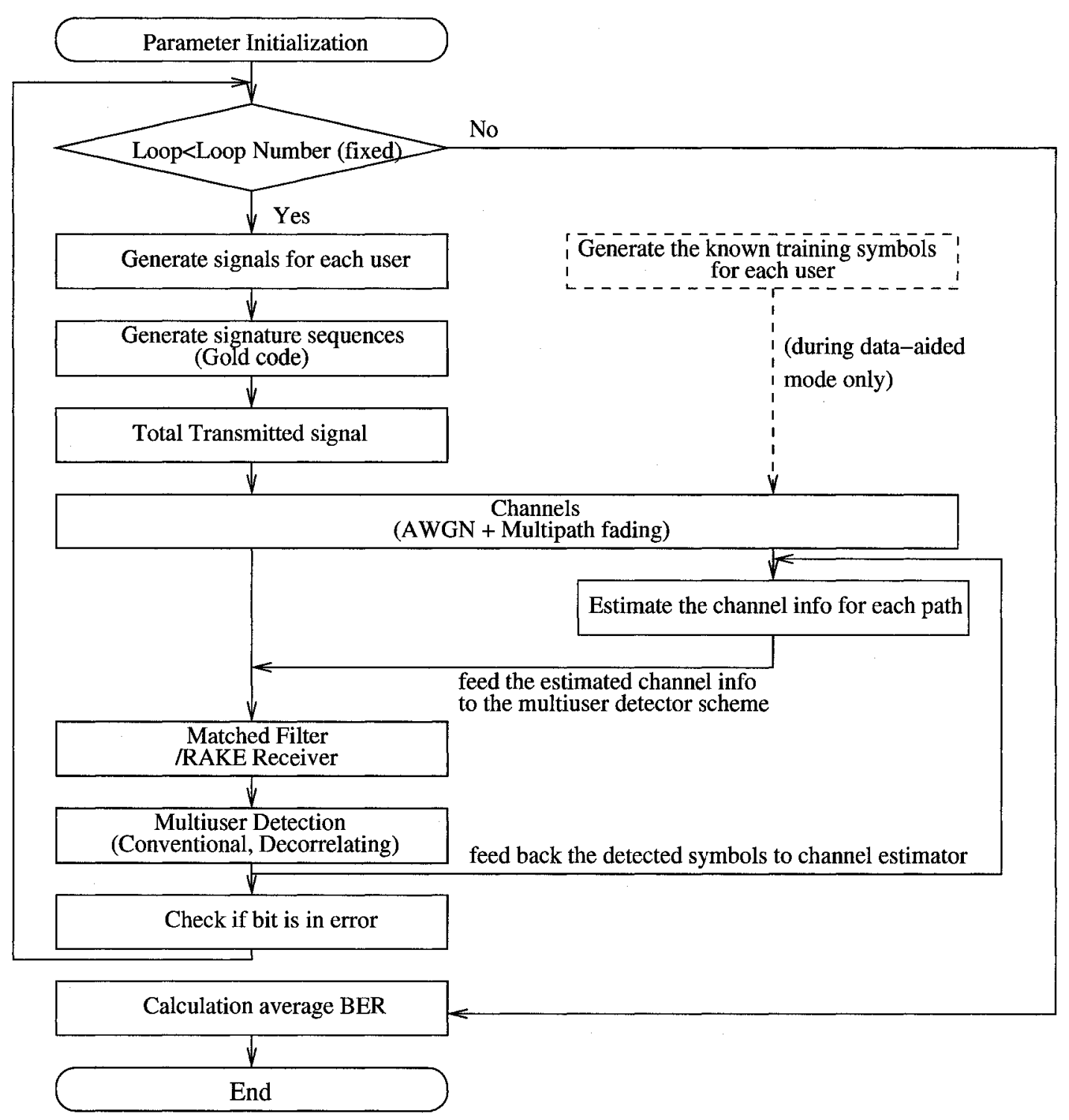

Fig. 6.2 Flowchart for the simulation code with channel estimation scheme implemented 


\subsection{Code verification}

For code verification purpose, first a system consisting of 10 single rate users over an AWGN channel was simulated. The obtained simulation results for single user bound, the conventional MF detector and the decorrelating detector are depicted in Fig. 6.3 where the MF output is plotted as an upper bound and single user BPSK output plotted as a lower bound for the performance curves. The plot illustrates the BER performance gains achieved by the decorrelating detector compared to that of the MF detector as $E_{b} / N_{0}$ increases. For example the gain is approximately $3 \mathrm{~dB}$ when BER is $2 \times 10^{-3}$. Similar results for this scenario are presented in [55].

Fig. 6.4 shows the simulation results for almost the same configuration and scenario as previously except that Rayleigh fading is employed in this case. It is observed that BER performance is degraded for both the decorrelating and MF detectors as well as single user bound due to the channel fading, but the decorrelating detector still outperforms the conventional MF detector significantly. The decorrelating detector yields a bigger performance gain over the MF detector simply because the decorrelating detector scheme is robust to channel fading and does not depend on the signal's transmitting power or the channel information in the case of perfect channel estimation. 


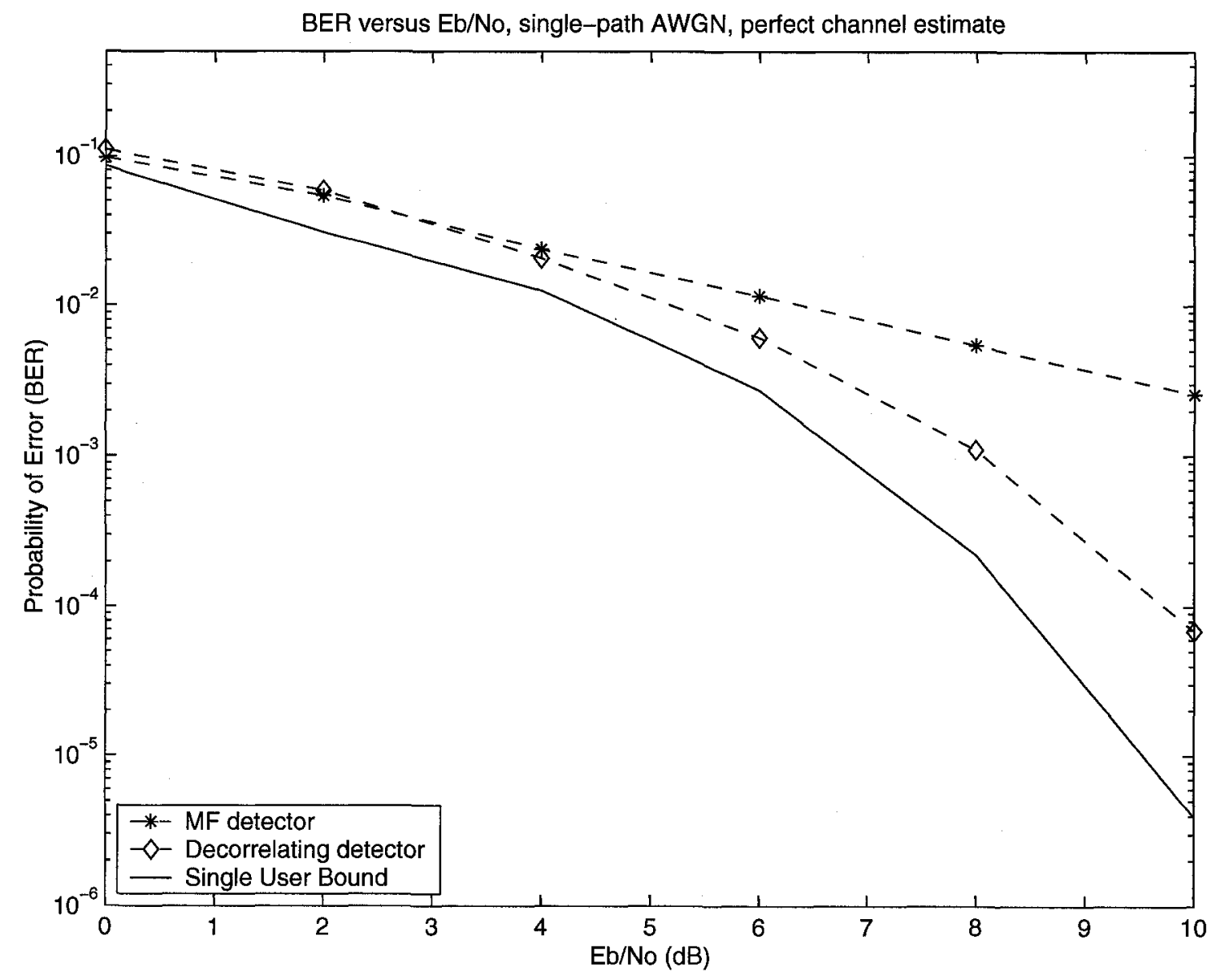

Fig. 6.3 BER performance under single-path AWGN channel, 10 single-rate users with processing gain of 32 


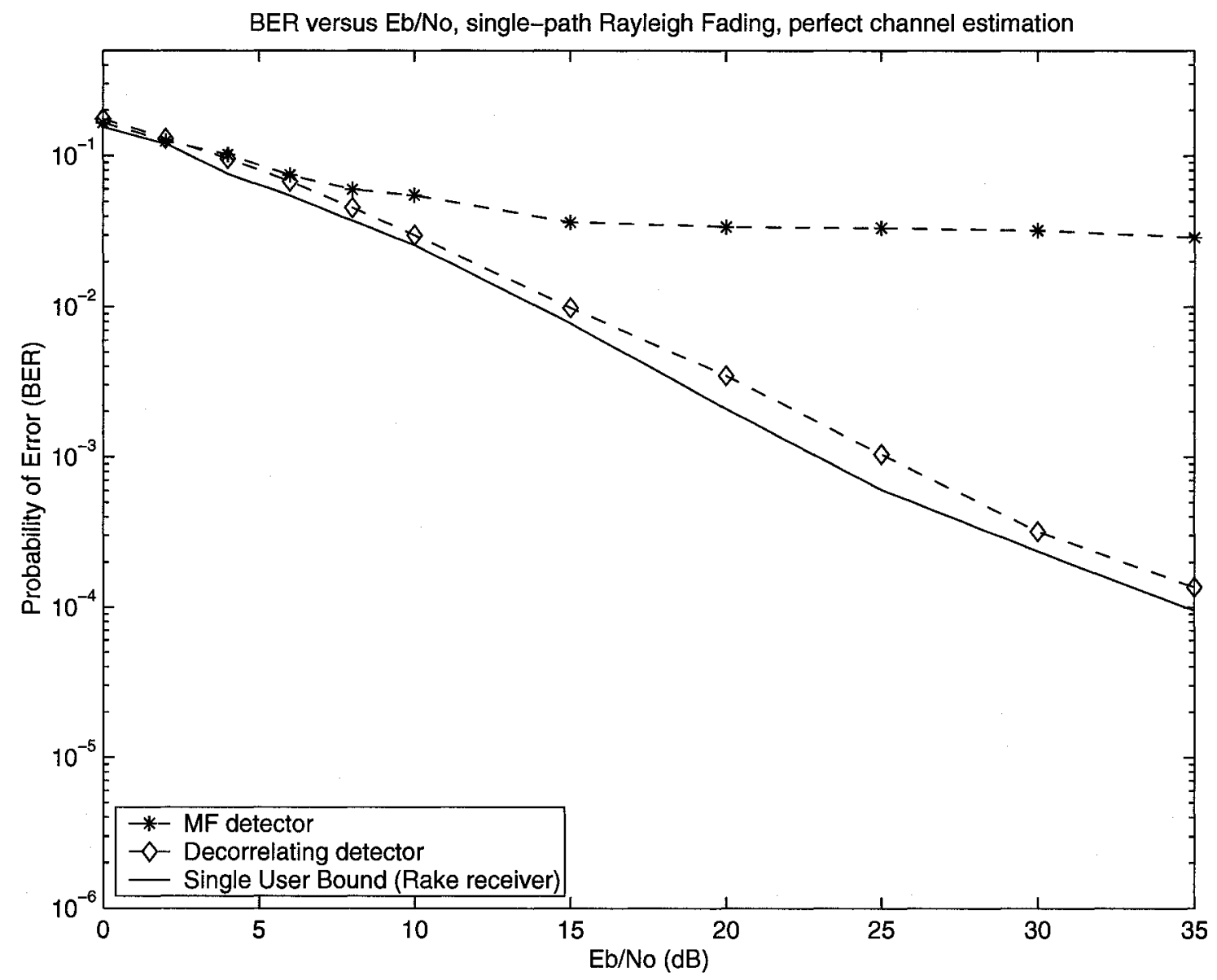

Fig. 6.4 BER performance under single-path Rayleigh Fading channel, 10 singlerate users with processing gain of 32

\subsection{Performance in the case of perfect channel estimation}

\subsubsection{Effect of having multirate users}

An asynchronous multirate DS-CDMA system with the same number of physical users (ten for multi-rate systems) is modeled in the simulations according to the cases outlined in Table 6.1. Case 1 corresponds to 10 single-rate users, while for case 4 , the system has 4 users in class 1 (of rate $R$ ), 3 users in class 2 (of rate $2 R$ ), 2 users in class 
3 (of rate $3 R$ ), 1 user in class 4 (of rate $4 R$ ). The processing gain of the lowest-rate users is 32 .

Table 6.1 Simulated physical users rates

\begin{tabular}{|r||r|r|r|r|r|}
\hline case & Class1 & Class2 & Class3 & Class4 & virtual users \\
\hline 1 & 10 & 0 & 0 & 0 & tot. \#=10 \\
2 & 7 & 3 & 0 & 0 & tot. \#=13 \\
3 & 5 & 3 & 2 & 0 & tot. \# =17 \\
4 & 4 & 3 & 2 & 1 & tot. \#=20 \\
\hline
\end{tabular}

Figs. 6.5-6.8 present performance of the conventional MF, decorrelating, MMSE, conventional SIC, PIC, decorrelating DF and DBSIC detectors over 2-path equal strength Rayleigh fading channels under perfect channel side information with various data rate classes as specified in Table 6.1 (from case 1 to case 4 ). It is seen that among all detectors, DBSIC achieves the best BER performance for both single and multirate systems, yielding for example compared to the decorrelating DF detector, a gain of $4.8 \mathrm{~dB}$ at a $10^{-5} \mathrm{BER}$ in a single-rate system (Fig. 6.5), $2.8 \mathrm{~dB}$ at a $2 \cdot 10^{-5} \mathrm{BER}$ in a 2-rate system (Fig. 6.6), 3dB at a $2 \cdot 10^{-5}$ BER in a 3-rate system (Fig. 6.7) and 2.2dB at a $4 \cdot 10^{-5} \mathrm{BER}$ in a 4-rate system (Fig. 6.8). Higher gains are obtained compared to the decorrelating detector $\left(6.2 \mathrm{~dB}\right.$ at a $2 \cdot 10^{-5} \mathrm{BER}$ in a single-rate system, $4.8 \mathrm{~dB}$ at a $3 \cdot 10^{-5} \mathrm{BER}$ in a 2-rate system), or the MMSE detector (4.5dB at a $4 \cdot 10^{-5} \mathrm{BER}$ in a 3 -rate system and $4.7 \mathrm{~dB}$ at a $8 \cdot 10^{-5} \mathrm{BER}$ in a 4 -rate system).

DBSIC also outperforms greatly the conventional SIC. This can be explained by the fact that SIC makes decisions based on the MF outputs which are then used for the next user detection and the decision estimate accuracy might be poor in the presence of MAI. DBSIC has more accurate decision estimate due to its decorrelating operation at each interference cancellation stage. The accuracy of the decision estimate is crucial to the overall system performance. However, the conventional SIC makes decisions based on MF outputs which is not good at all especially when SNR is 


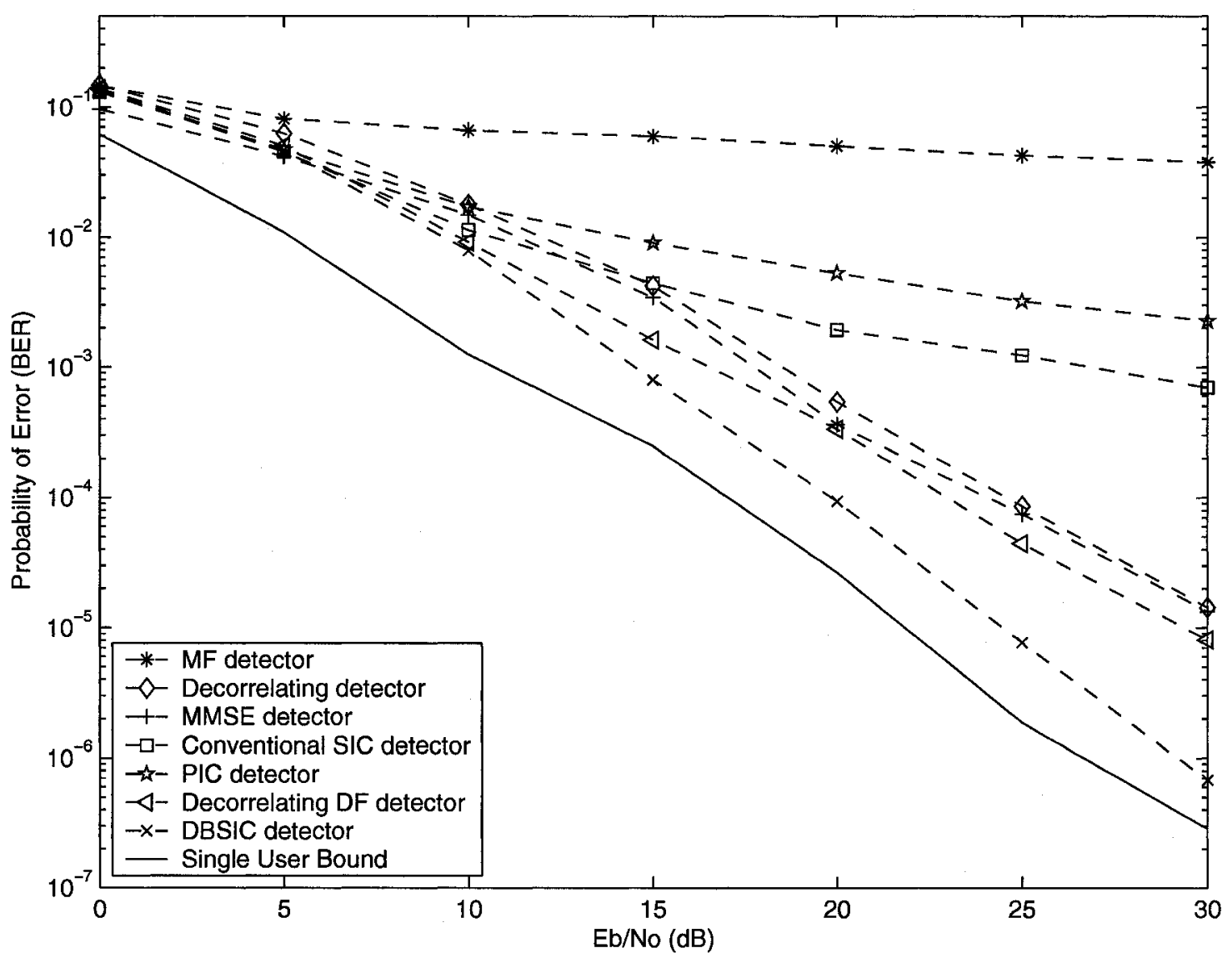

Fig. 6.5 Performance over 2-path Rayleigh fading channel with RAKE, perfect channel estimation, $K_{1}=10, G_{1}=32$

high. It is observed from Figs. 6.3-6.4 that the decorrelating detector has much better performance than the MF one, especially at high SNR. Therefore, with the combination of decorrelating detector and SIC structure, the DBSIC detector exploits the advantages of both schemes and boosts the overall system's performance with feasible computational complexity. As DBSIC conducts the detection in the order of received signals energy/amplitude (based on the outputs of the bank of MF), those users with higher received SNR would be determined first. The initial decision estimate would be more accurate as well due to the fact that a decorrelator is implemented in each 


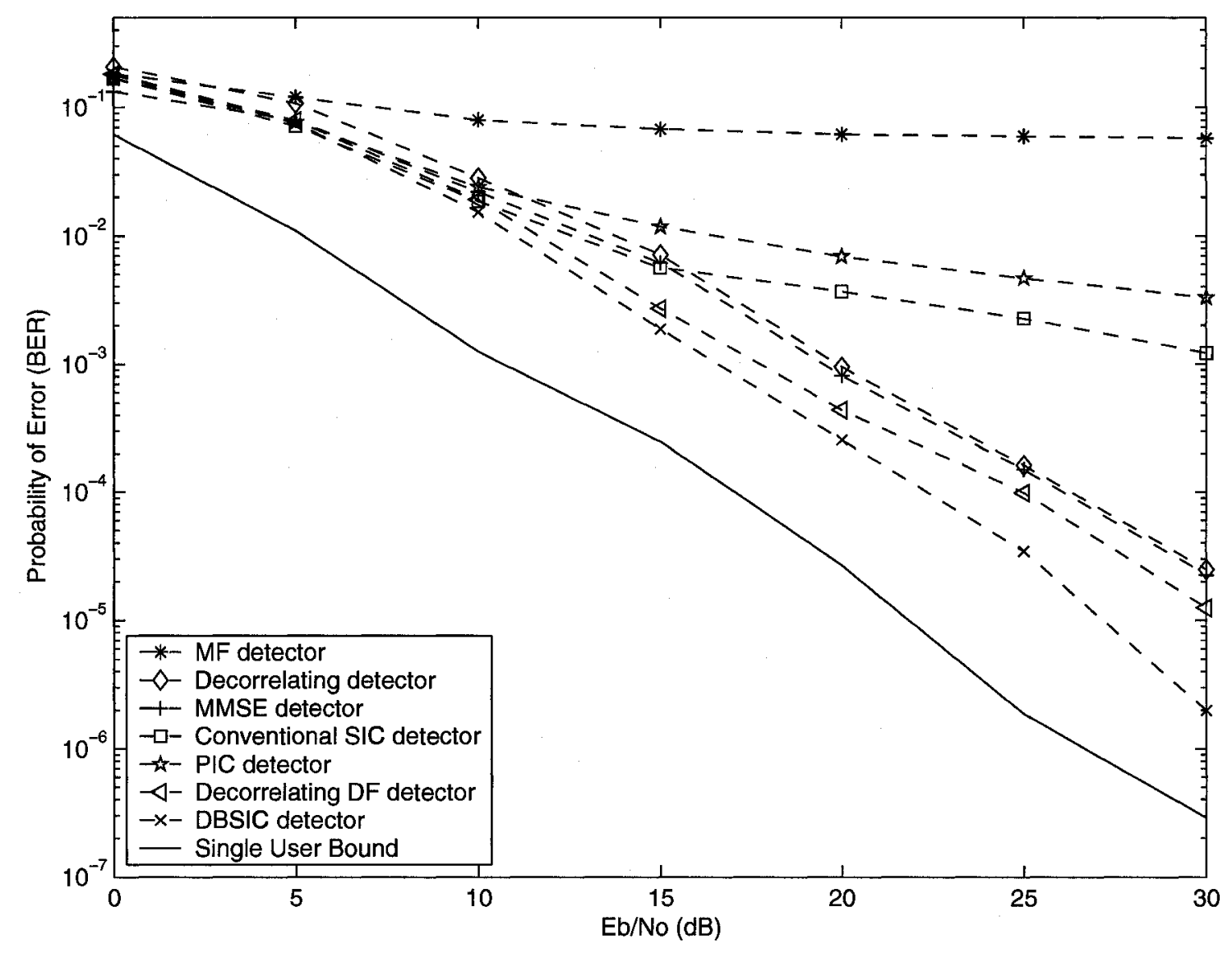

Fig. 6.6 Performance over 2-path Rayleigh fading channel with RAKE, perfect channel estimation, $K_{1}=7, K_{2}=3, G_{1}=32$

stage of DBSIC. This explains the dramatically improved performance of the proposed detector structure compared to the conventional SIC. It is also observed that the performance differences for all detectors are subtle at low $E_{b} / N_{0}$ and become significant as $E_{b} / N_{0}$ increases. This is because the noise term dominates when $E_{b} / N_{0}$ is small no matter what detector structure is implemented. As $E_{b} / N_{0}$ increases, the interference term including MAI and ISI overweights the noise term gradually and the DBSIC demonstrates its capability to improve the system performance over the existing known multi-user detectors. 


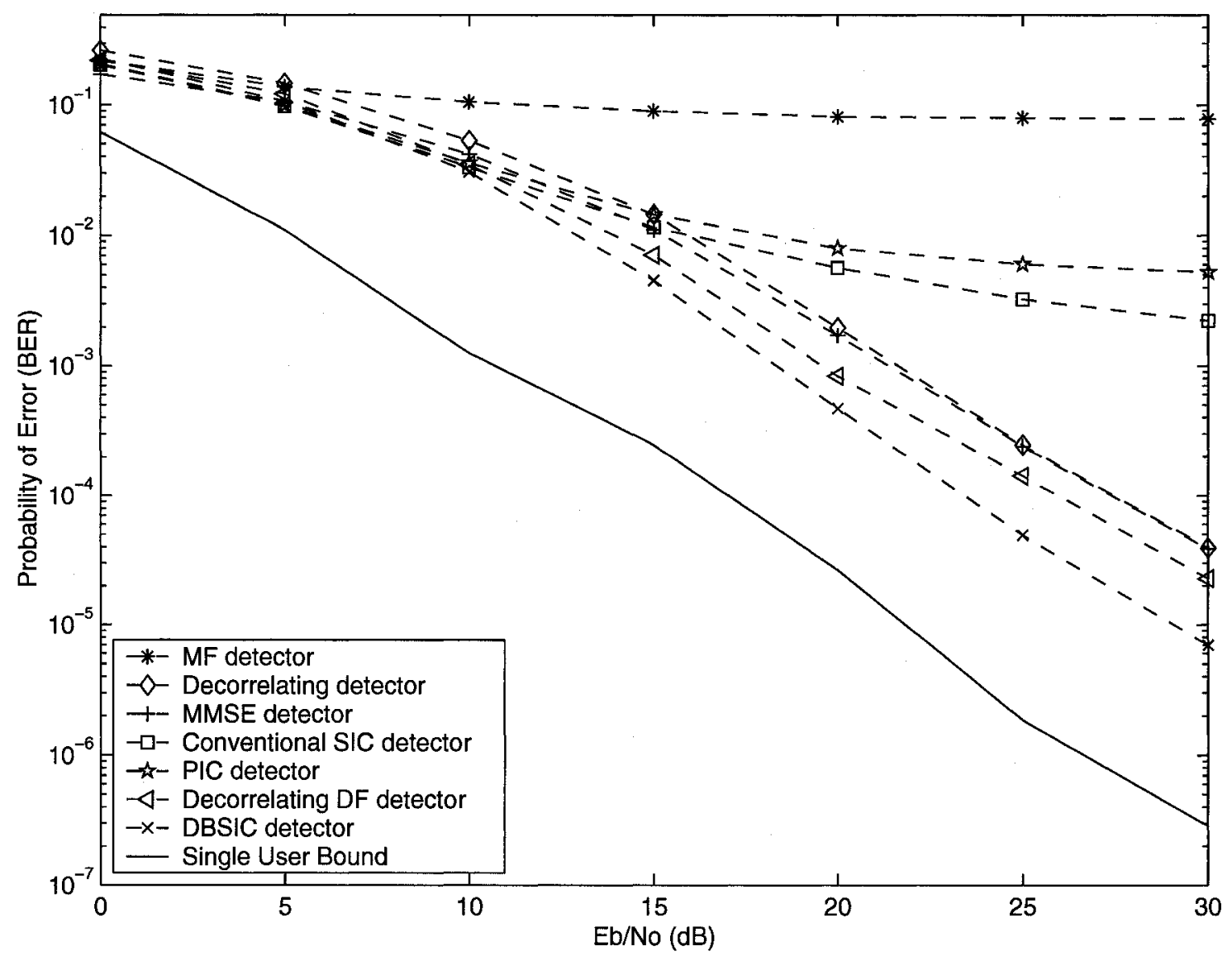

Fig. 6.7 Performance over 2-path Rayleigh fading channel with RAKE, perfect channel estimation, $K_{1}=5, K_{2}=3, K_{3}=2, G_{1}=32$

As more and more users demand higher data rate such as from case 1 to case 4 , the number of virtual users increases accordingly and yields performance loss for all receivers as expected (heavier loaded system case). However, Fig. 6.8 shows that DBSIC's performance degradation is smaller. For example, Fig. 6.8 shows a penalty of $2.2 \mathrm{~dB}$ for DBSIC at a $2 \cdot 10^{-6}$ BER when some physical users rates double from the original single-rate system. The performance degrades by an additional $3.4 \mathrm{~dB}$ in the 3-rate case where the data rate triples. 


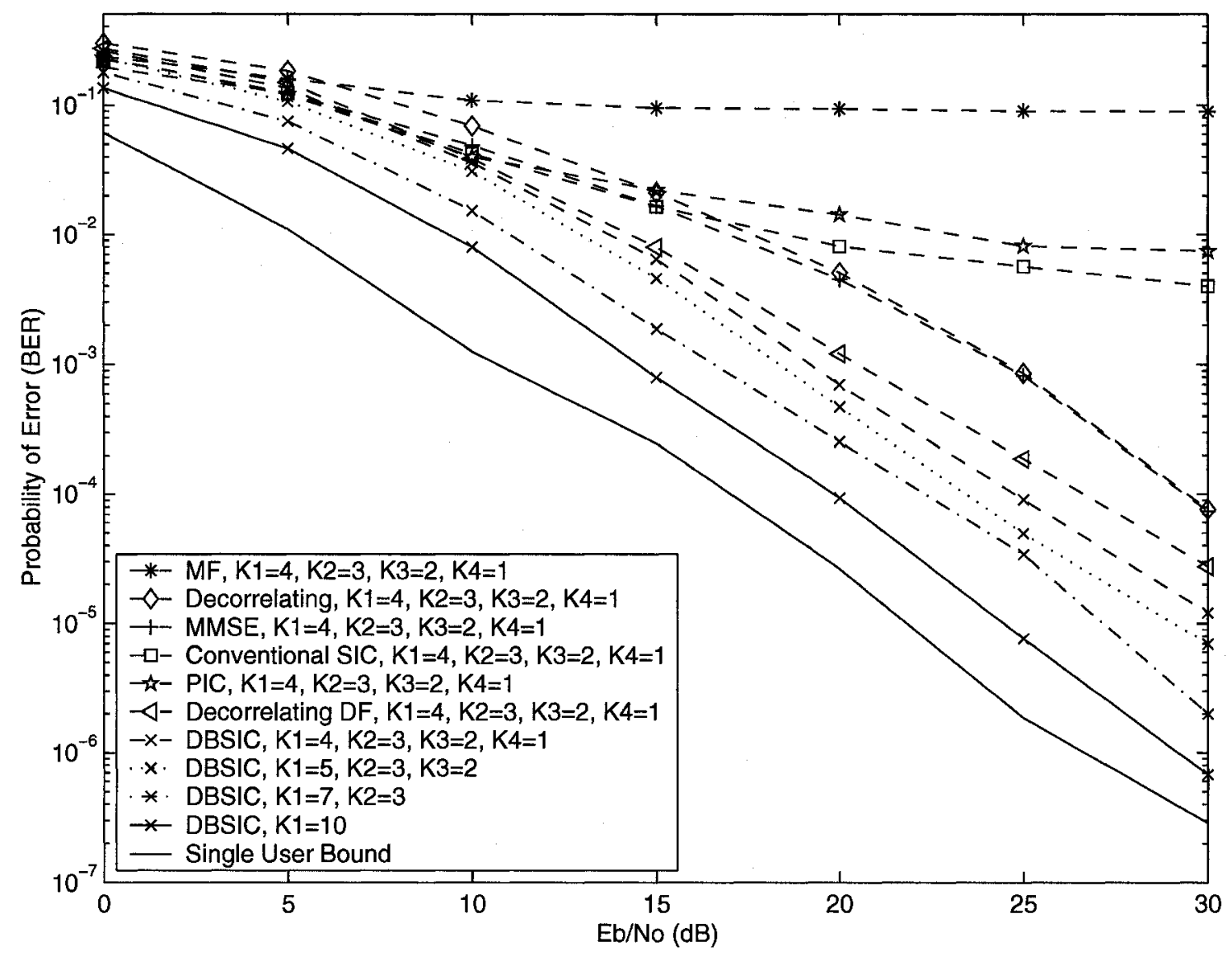

Fig. 6.8 Performance over 2-path Rayleigh fading channel with RAKE, perfect channel estimation, single and multi-rates, $G_{1}=32$

\subsubsection{Performance of best and worst users}

The results presented in Figs 6.5-6.8 are all based on the BER performance averaged over all users in the system, which will be referred to as "cumulative BER performance". Figs. 6.9-6.12 show both the cumulative BER performance for the system and BER for the users with best and worst performance (denoted as "best performance" and "worst performance", respectively). It can be observed that even the user with worst performance for DBSIC achieves the best BER performance among 


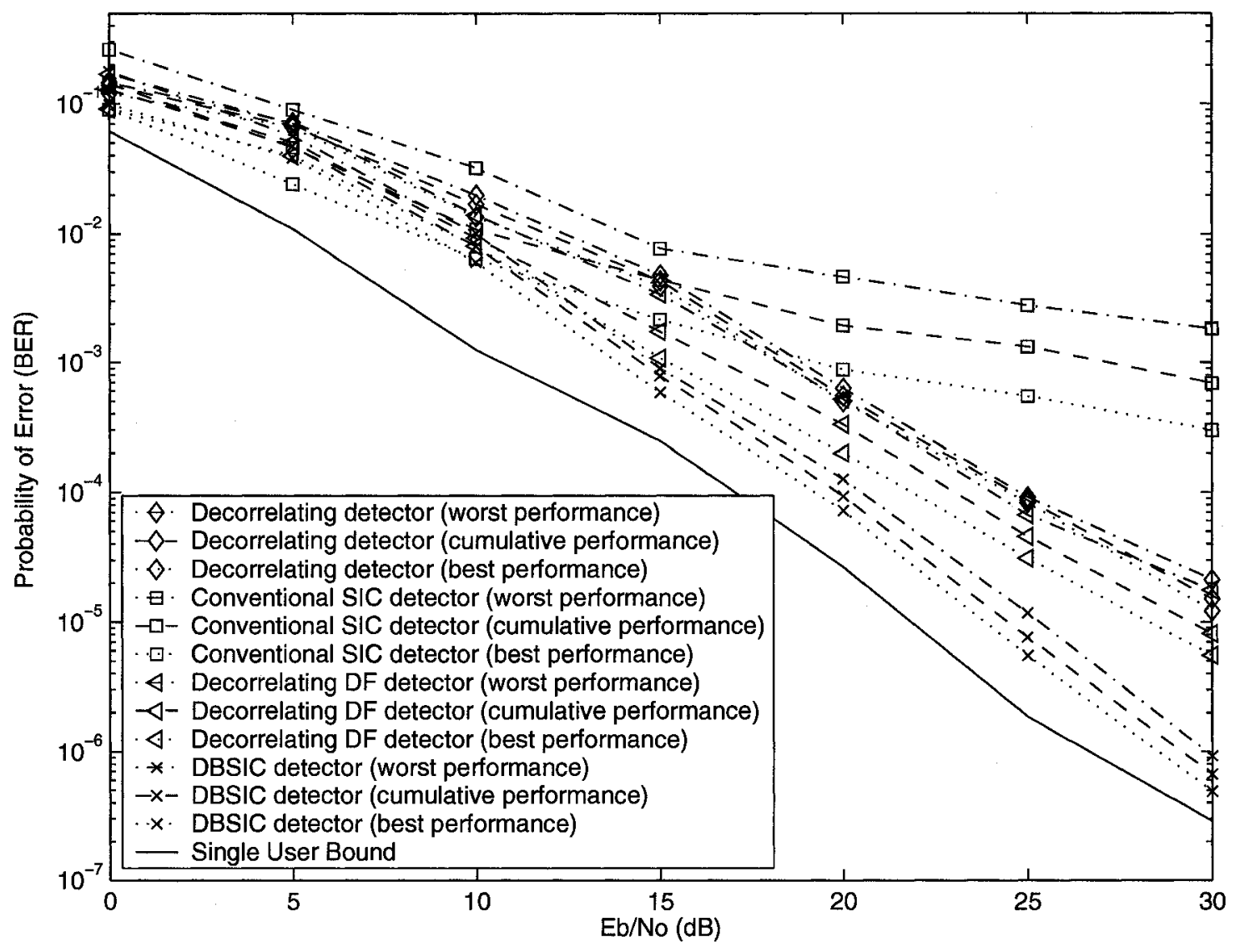

Fig. 6.9 Performance over 2-path Rayleigh fading channel with RAKE, perfect channel estimation, $K_{1}=10, G_{1}=32$

all detectors for both single and multirate systems, yielding compared to the decorrelating DF detector, a gain of $3.0 \mathrm{~dB}$ at a $10^{-5}$ BER in a single-rate system (Fig. 6.9), $1.5 \mathrm{~dB}$ at a $2 \cdot 10^{-5} \mathrm{BER}$ in a 3-rate system (Fig. 6.11) and compared to the MMSE detector, a gain of $3.2 \mathrm{~dB}$ at a $2 \cdot 10^{-5} \mathrm{BER}$ in a 2-rate system (Fig. 6.11) and 3.6dB at a $10^{-4} \mathrm{BER}$ in a 4 -rate system (Fig. 6.12).

Figs.6.5-6.12 previously presented assume that the cancellation order of the conventional SIC and DBSIC is determined based on the outputs of the bank of matched filters as explained in section 3.3. In the next section, we will investigate the effect of 


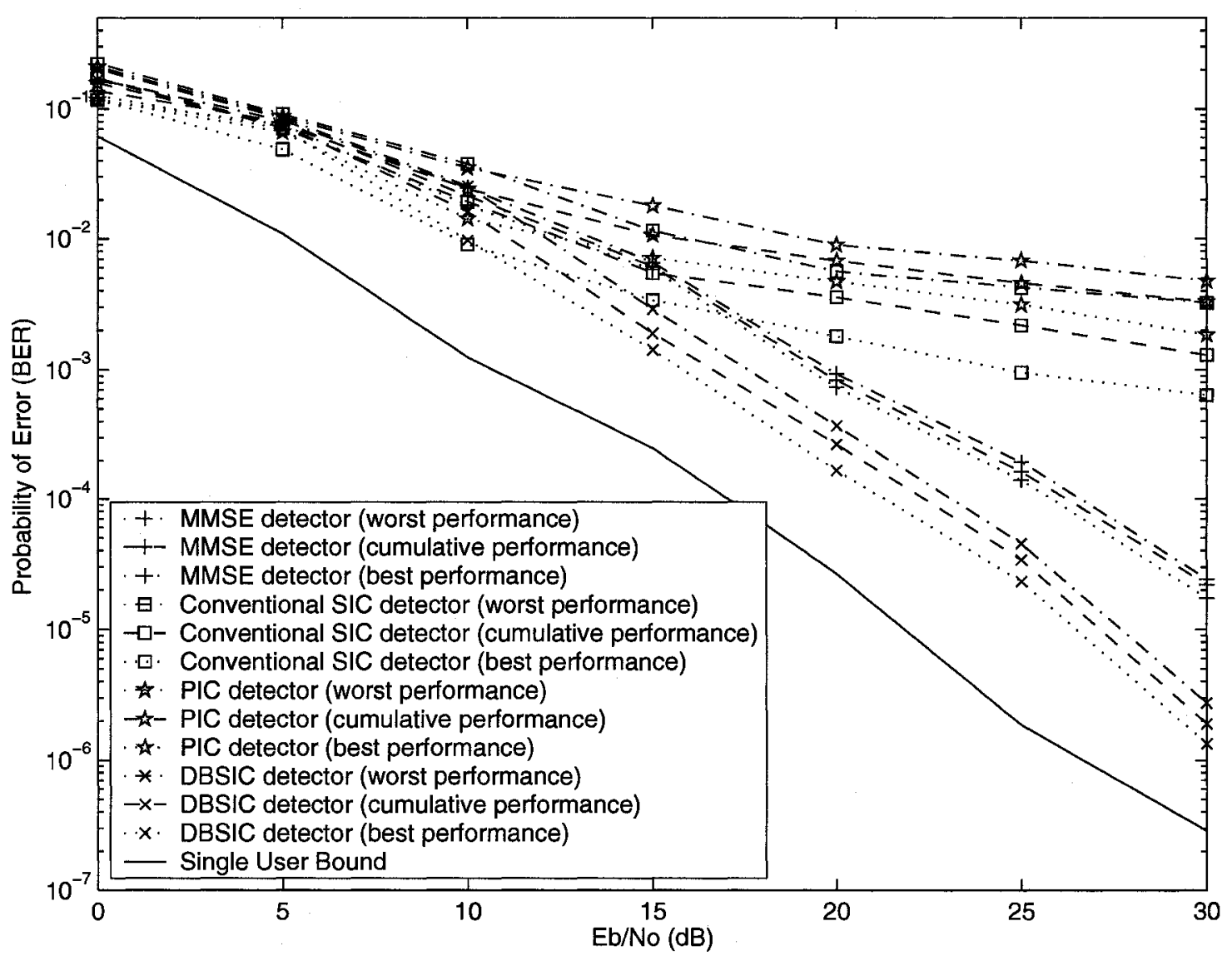

Fig. 6.10 Performance over 2-path Rayleigh fading channel with RAKE, perfect channel estimation, $K_{1}=7, K_{2}=3, G_{1}=32$

the order of user cancellation on the performance of conventional SIC and DBSIC. 


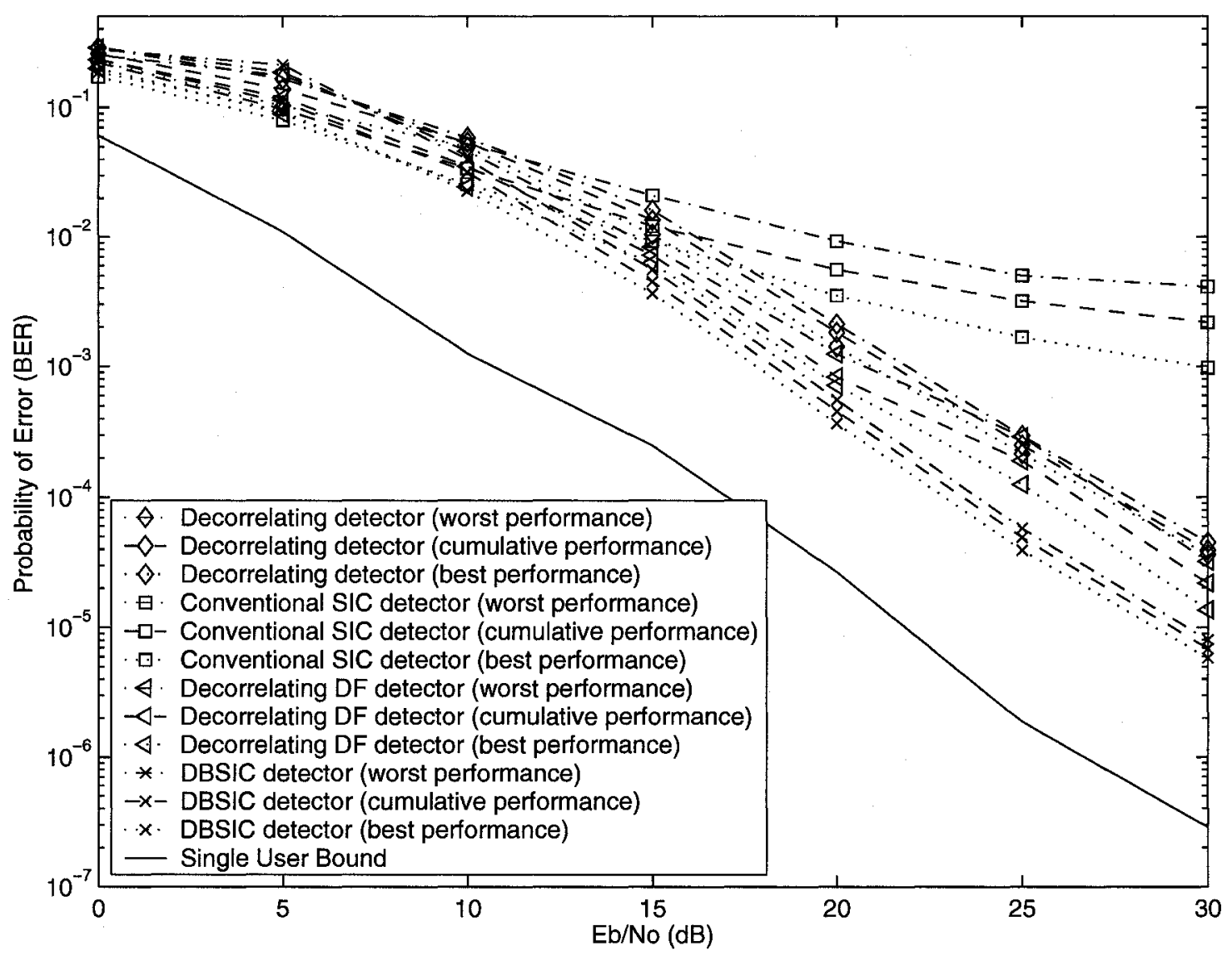

Fig. 6.11 Performance over 2-path Rayleigh fading channel with RAKE, perfect channel estimation, $K_{1}=5, K_{2}=3, K_{3}=2, G_{1}=32$ 


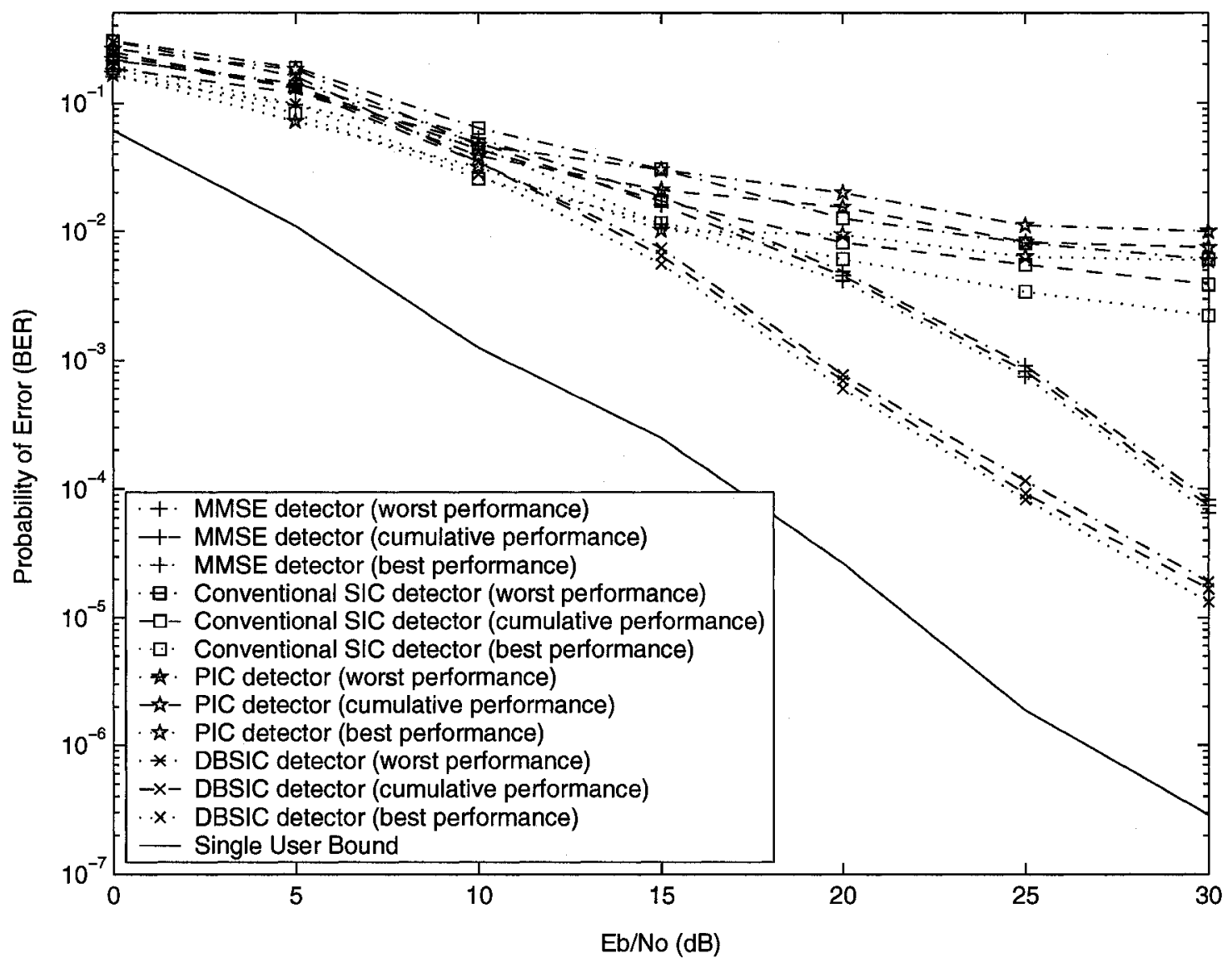

Fig. 6.12 Performance over 2-path Rayleigh fading channel with RAKE, perfect channel estimation, $K_{1}=4, K_{2}=3, K_{3}=2, K_{4}=1, G_{1}=32$

\subsubsection{Effect of user cancellation order}

A dual-rate system with 3 users in class $1\left(K_{1}=3\right)$ and two users in class $2\left(K_{2}=2\right)$ yielding a total number of 7 virtual users is considered. The processing gain of the lowest rate users is 32 . Fig. 6.13 presents the performance of the conventional MF detector, decorrelating detector, conventional SIC and proposed DBSIC detectors with random user cancellation order, conventional SIC and DBSIC detectors with user cancellation based on MF outputs (as explained in section 3.3). Among all 
schemes, the DBSIC detector achieves the best BER performance regardless of the user cancellation/detecting order it uses, and outperforms significantly the conventional SIC detector.

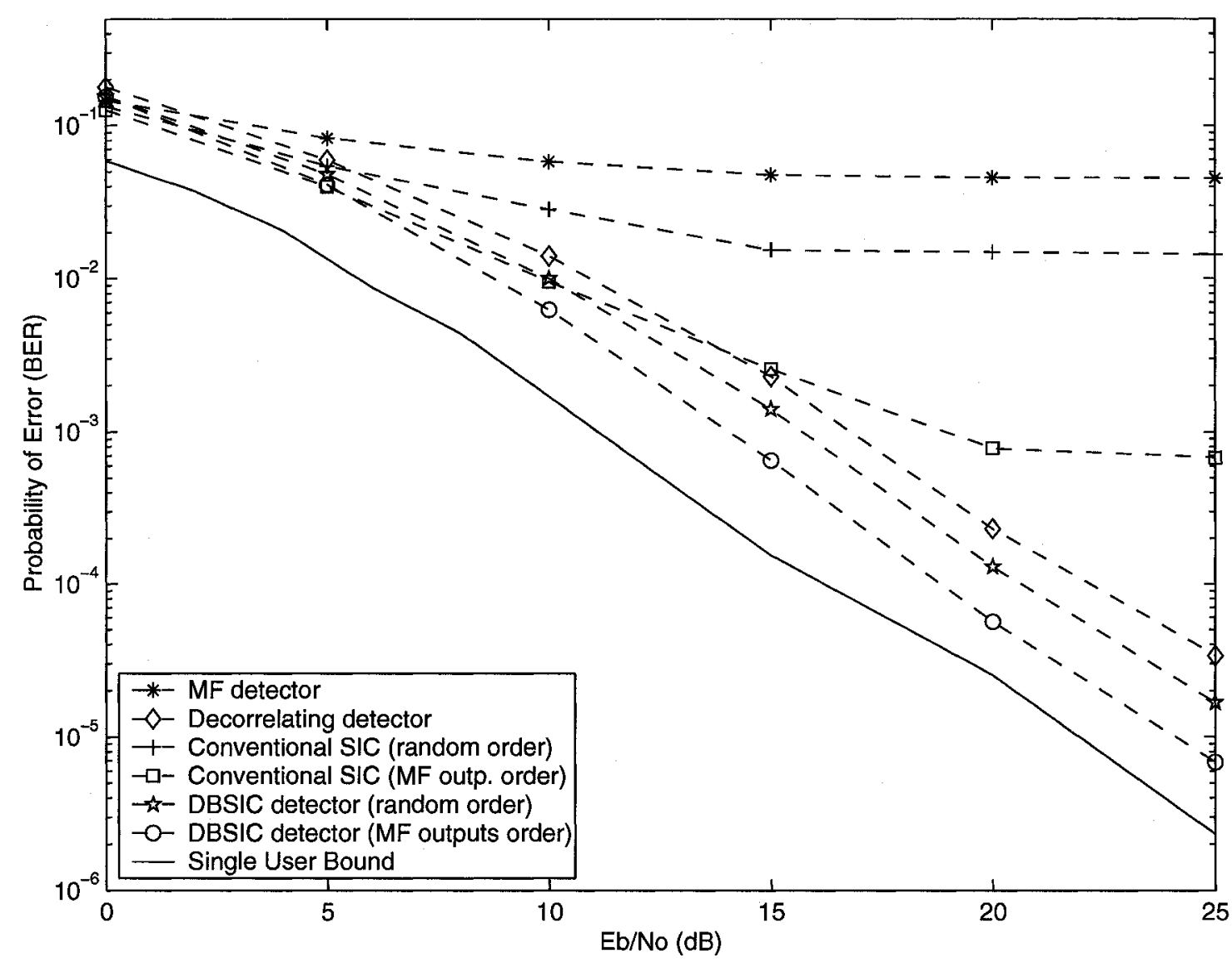

Fig. 6.13 Performance over 2-path Rayleigh fading channel with RAKE, perfect channel estimation, $K_{1}=3, K_{2}=2, G_{1}=32$ (MF outputs/random user cancellation order)

It is seen from Fig. 6.13 that SIC performance is quite sensitive to the user cancellation order having a quite high error floor in the case of random ordering, whereas DBSIC still achieves diversity gains even with random ordering. One way to improve performance of the conventional SIC is to use it in combination with a non-uniform 
power control system [92, 93, 94]. Given that DBSIC also uses successive interference cancellation strategy, the techniques proposed in $[92,93,94]$ could be modified to further enhance the performance of DBSIC as well. As seen in chapter 5, and confirmed by the simulation results, DBSIC is more near-far resistant than the MF and conventional SIC detectors, and is less sensitive to the user cancellation order than SIC. Therefore, it could be inferred that in the event where a power control scheme is used with DBSIC, DBSIC would be less sensitive to imperfect power control than the MF or conventional SIC schemes.

When comparing DBSIC with other commonly used multiuser detectors in terms of BER, the same effects, observed in the dual rate case presented in Fig. 6.6, are observed in Fig. 6.13 (which also considers a dual rate case but different from the one in Fig. 6.6). For example, Fig. 6.13 shows that DBSIC with user cancellation order based on MF outputs outperforms the decorrelating detector by approximately $4 \mathrm{~dB}$ at BER of $3 \cdot 10^{-5}$. The more striking result from Fig. 6.13 is that DBSIC with random user cancellation order can still offer $1.5 \mathrm{~dB}$ performance gain over the decorrelating detector. It is also observed that the DBSIC scheme has roughly $2.3 \mathrm{~dB}$ performance degradation compared to Rake receiver single user bound.

\subsubsection{Effect of assignment of Gold sequences to users}

The simulation results presented in Figs. 6.5-6.8 are based on using a fixed set of Gold sequences to generate the signature sequences of the virtual users using the second method described in section 3.1. In Figs. 6.14-6.17 a different set of Gold sequences is used to generate the signature sequences of the virtual users (still using the second method). Compared with Figs. 6.5-6.8, the results presented in Figs. 6.146.17 indicate that performance results are quite independent of the choice of Gold sequences. Figs. 6.16-6.17 show some slight variations of performance according to 
the choice of Gold sequences as the number of high rate users increases, but the trends and the orders of BER remain unchanged. In particular, regardless of the Gold sequences used, DBSIC still outperforms all the other multiuser detectors.

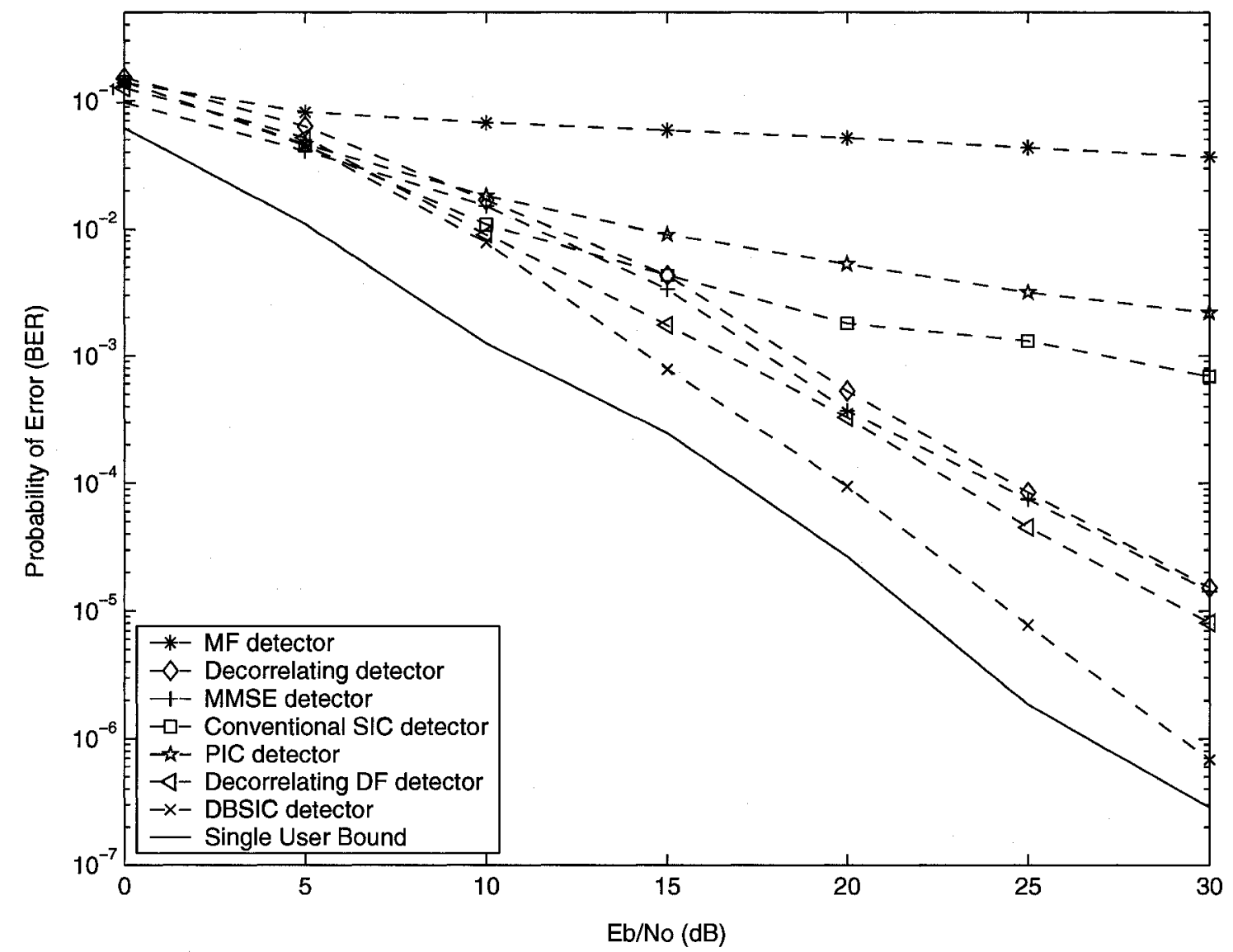

Fig. 6.14 Performance over 2-path Rayleigh fading channel with RAKE, perfect channel estimation, $K_{1}=10, G_{1}=32$, different set of Gold sequences 


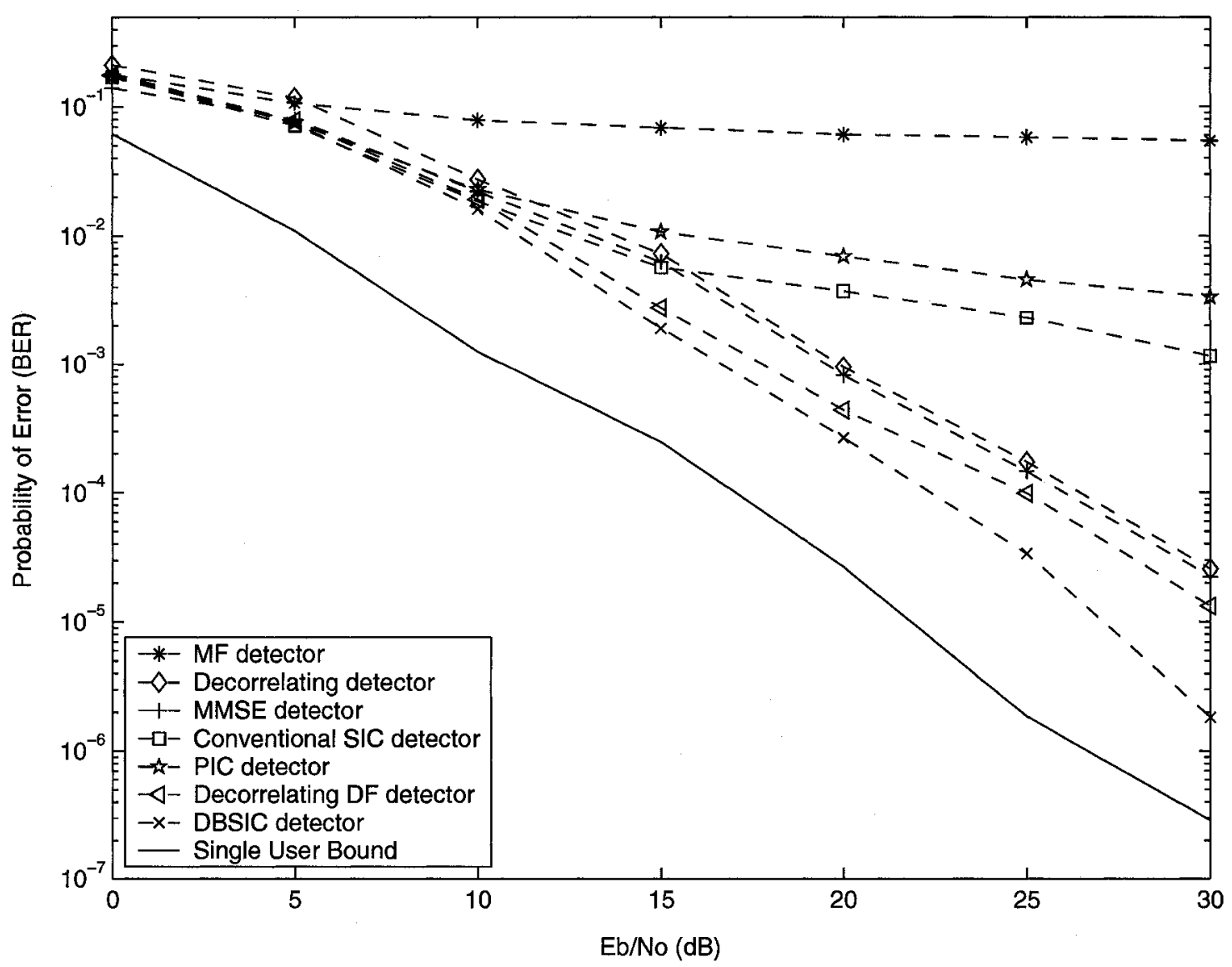

Fig. 6.15 Performance over 2-path Rayleigh fading channel with RAKE, perfect channel estimation, $K_{1}=7, K_{2}=3, G_{1}=32$, different set of Gold sequences 


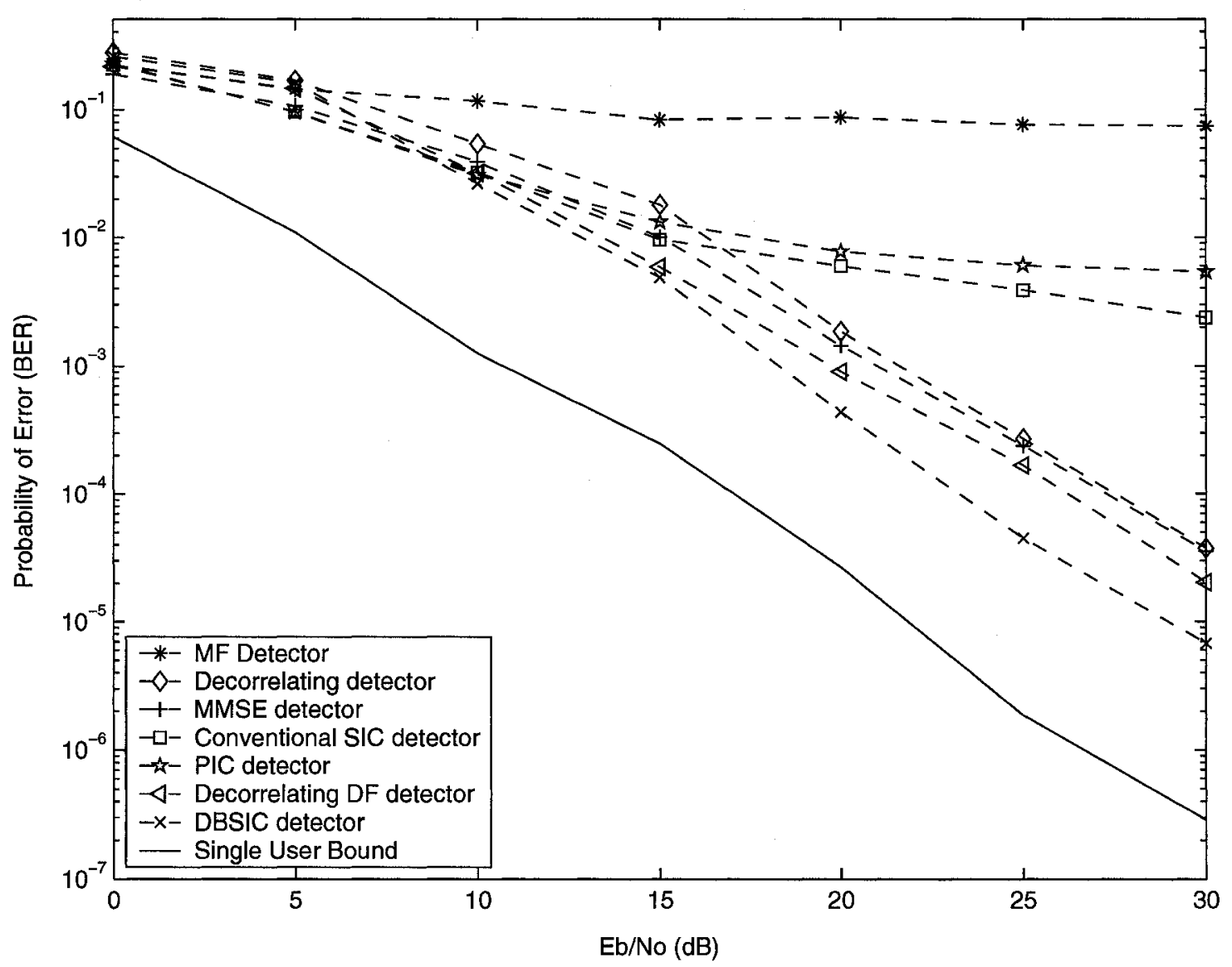

Fig. 6.16 Performance over 2-path Rayleigh fading channel with RAKE, perfect channel estimation, $K_{1}=5, K_{2}=3, K_{3}=2, G_{1}=32$, different set of Gold sequences 


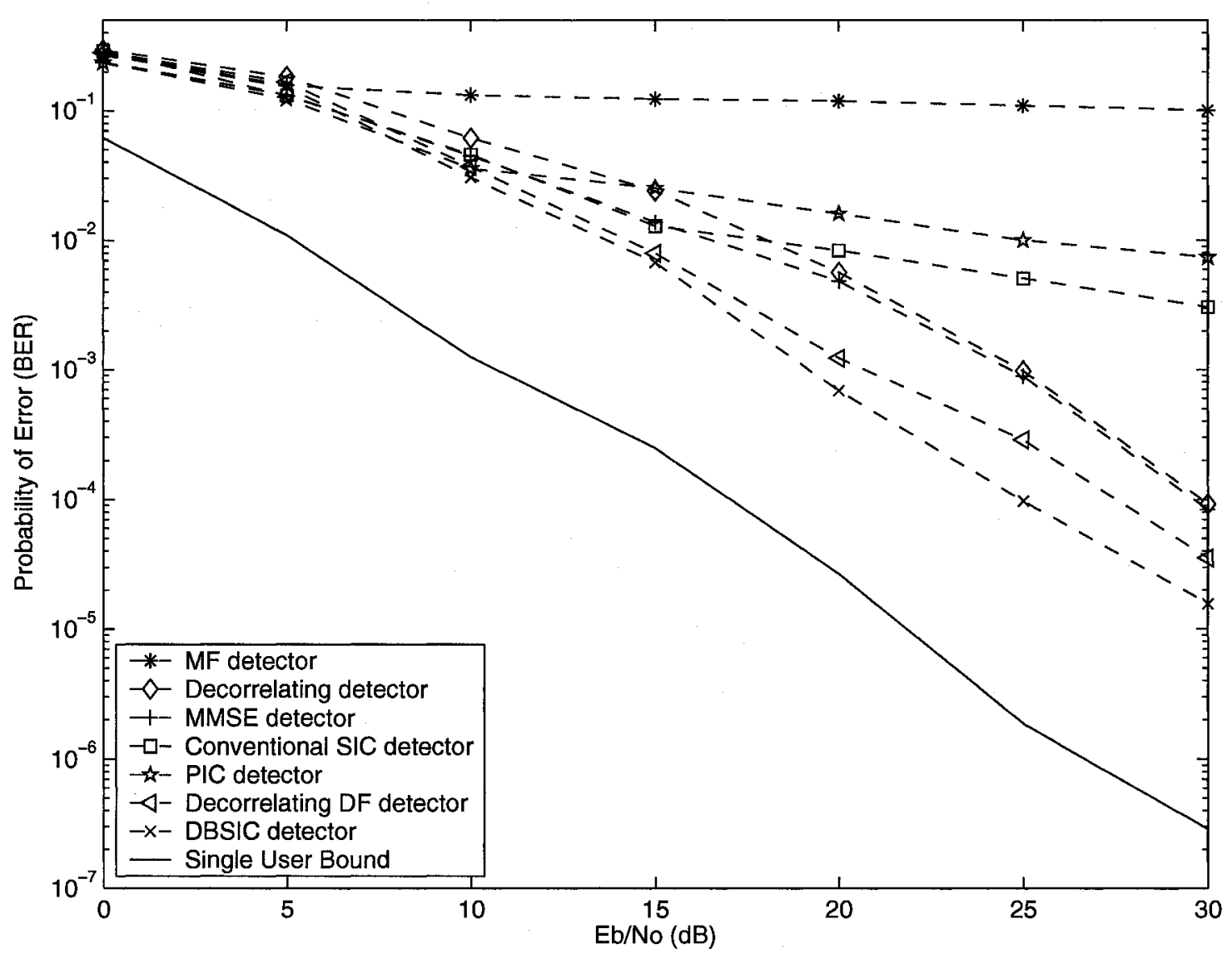

Fig. 6.17 Performance over 2-path Rayleigh fading channel with RAKE, perfect channel estimation, $K_{1}=4, K_{2}=3, K_{3}=2, K_{4}=1, G_{1}=32$, different set of Gold sequences

\subsubsection{Effect of the method of implementation of VPG}

Recall from section 3.1 that there are two ways of implementing a VPG DS-CDMA system or equivalently two ways of choosing the signature sequences (spreading codes) of the virtual users corresponding to one high rate physical user. So far, all results presented in this chapter were obtained using the second method. Figs. 6.18-6.21 show the performance results based on using the first method of implementing the VPG system (i.e. using (3.2)-(3.3) to generate the signature sequences of the virtual users). Comparing Figs. 6.18-6.21 with Figs. 6.5-6.8, it is observed DBSIC achieves 


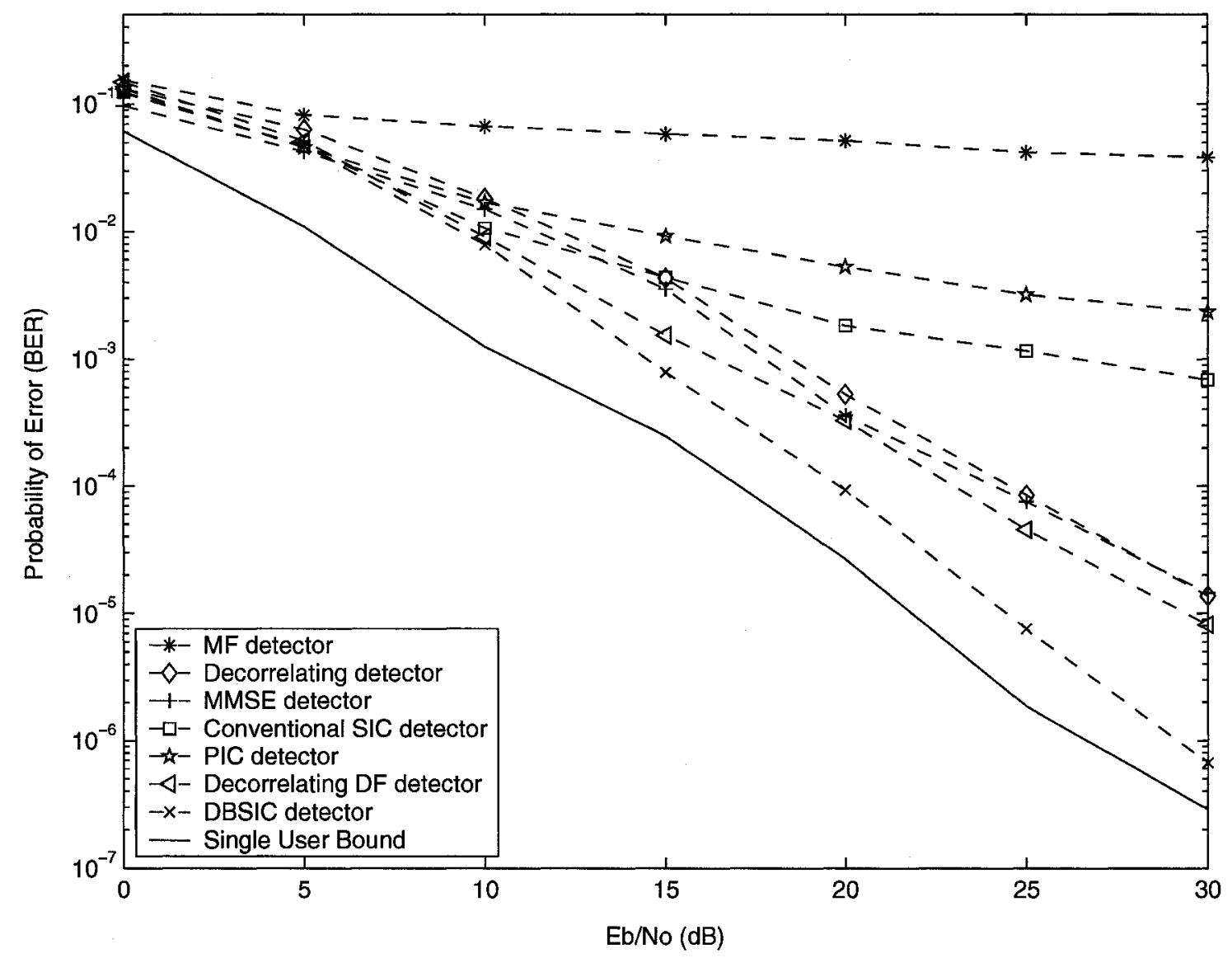

Fig. 6.18 Performance over 2-path Rayleigh fading channel with RAKE, perfect channel estimation, $K_{1}=10, G_{1}=32$, virtual users signatures sequences generated with the 1st method

similar BER performance improvement over the other multiuser detectors when the first implementation method of VPG is used. 


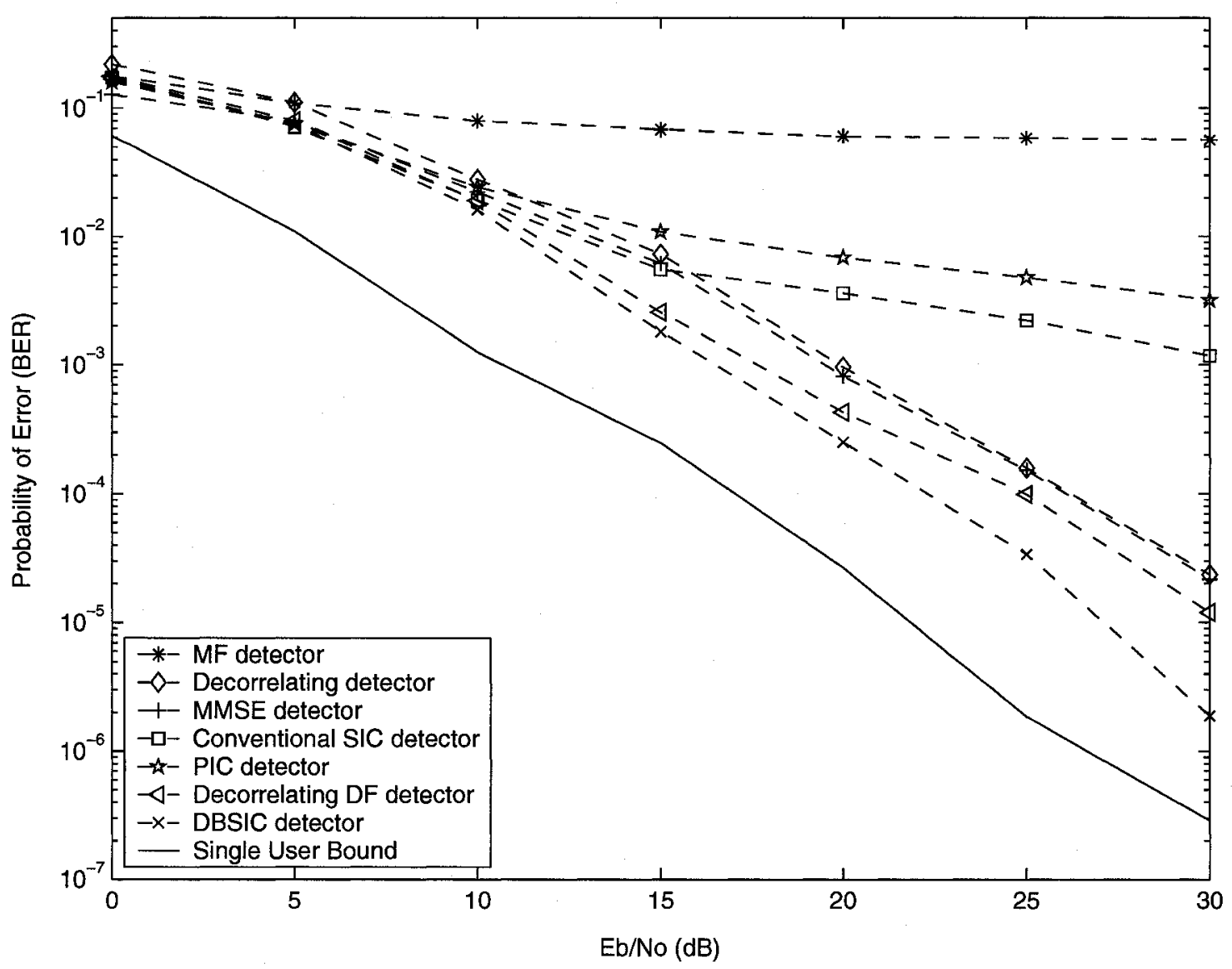

Fig. 6.19 Performance over 2-path Rayleigh fading channel with RAKE, perfect channel estimation, $K_{1}=7, K_{2}=3, G_{1}=32$, virtual users signatures sequences generated with the 1st method 


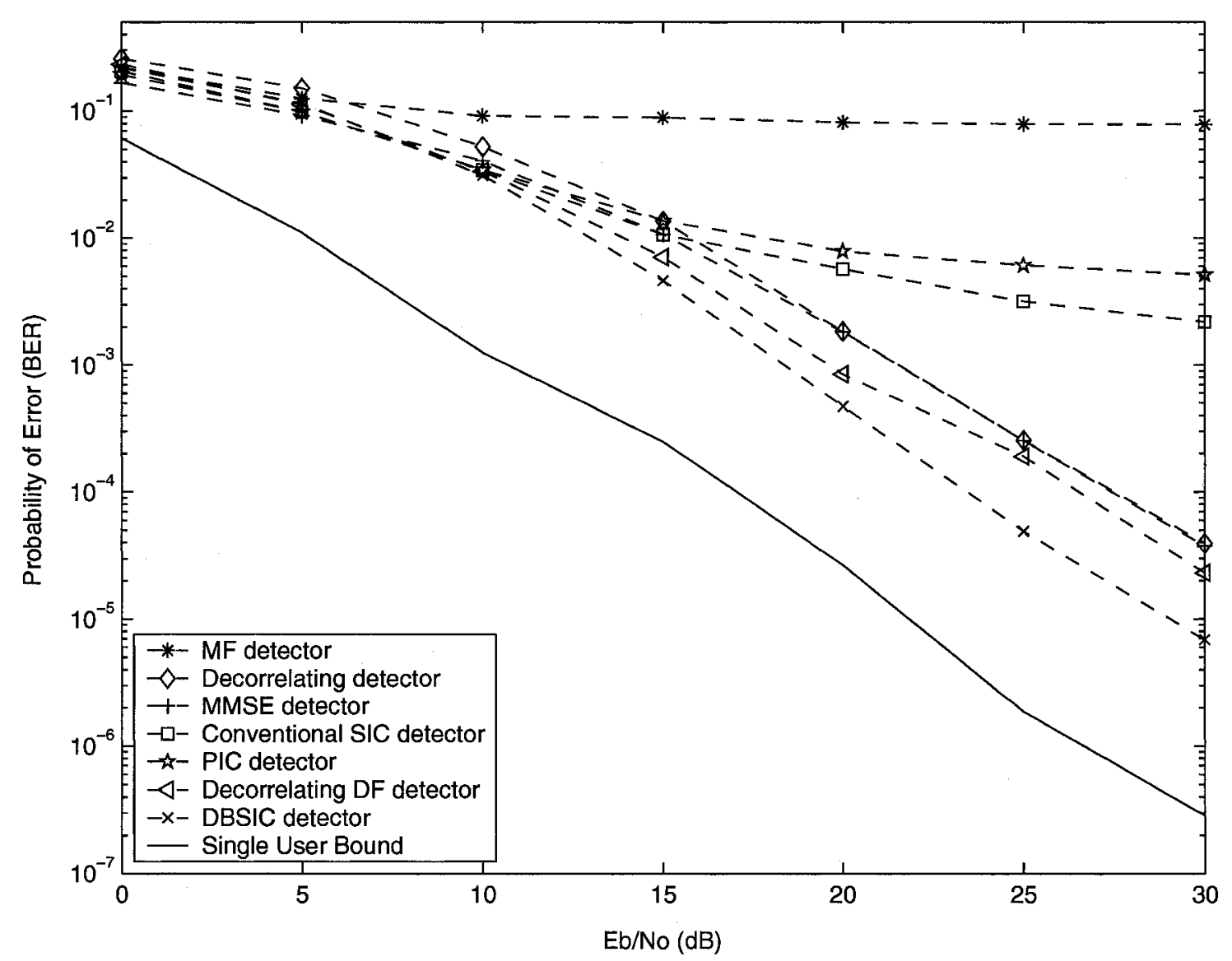

Fig. 6.20 Performance over 2-path Rayleigh fading channel with RAKE, perfect channel estimation, $K_{1}=5, K_{2}=3, K_{3}=2, G_{1}=32$, virtual users signatures sequences generated with the 1st method 


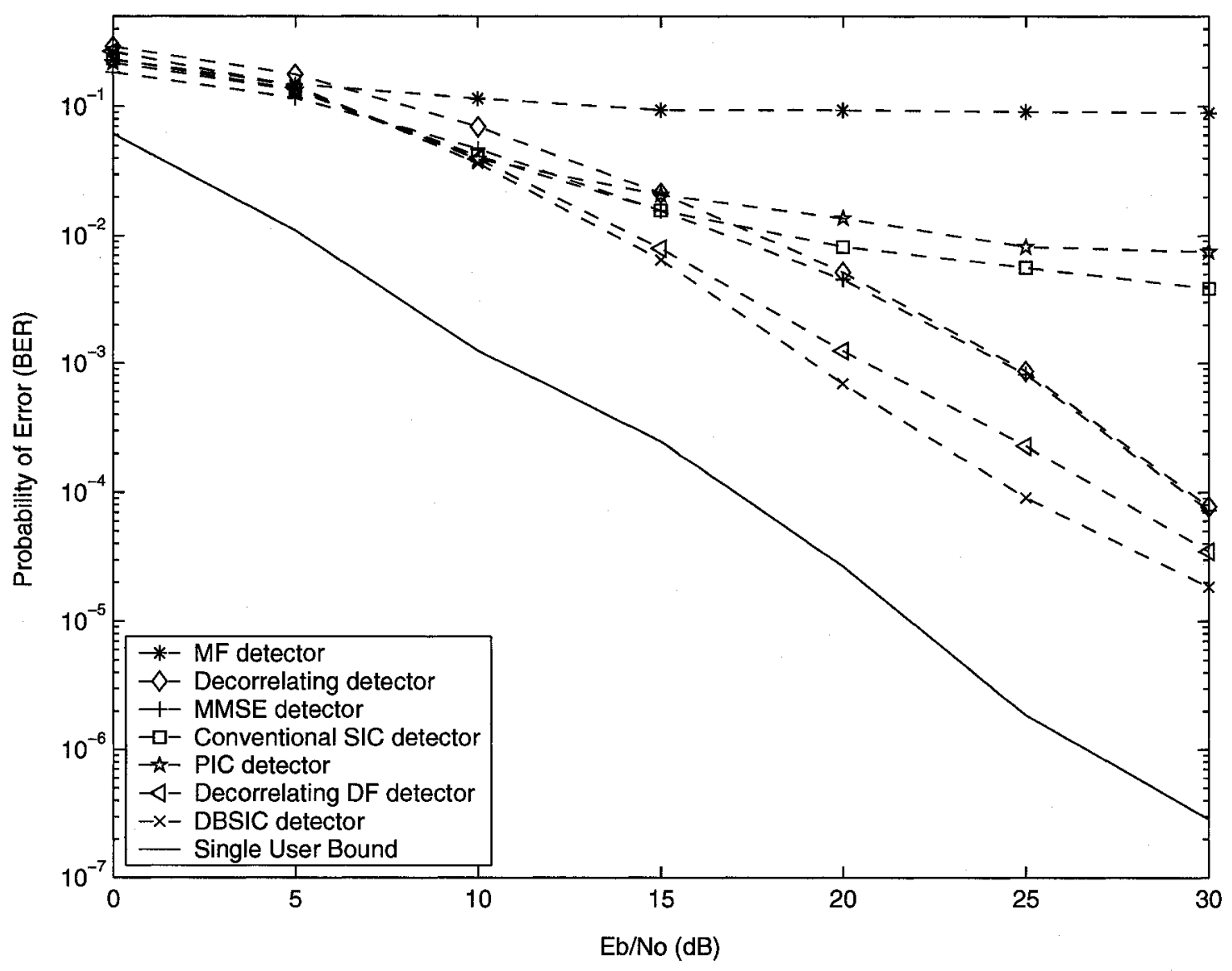

Fig. 6.21 Performance over 2-path Rayleigh fading channel with RAKE, perfect channel estimation, $K_{1}=4, K_{2}=3, K_{3}=2, K_{4}=1, G_{1}=32$, virtual users signatures sequences generated with the 1st method

\subsubsection{Effect of processing gain}

In Figs. 6.5-6.21, the processing gain for the lowest rate users is 32. Fig 6.22 presents BER performance for a 2-rate system with a processing gain of 64 for the lowest rate users. The total number of physical users is twenty with seventeen 1-rate users and three 2-rate users in the system. It is shown that DBSIC achieves the performance gain of $5.0 \mathrm{~dB}$ at a $10^{-5} \mathrm{BER}$ compared to the decorrelating $\mathrm{DF}$ detector, $5.0 \mathrm{~dB}$ at a $10^{-4} \mathrm{BER}$ compared to the decorrelating detector. Fig. 6.22 shows that DBSIC 
maintains its performance advantage over the other detectors for higher processing gains.

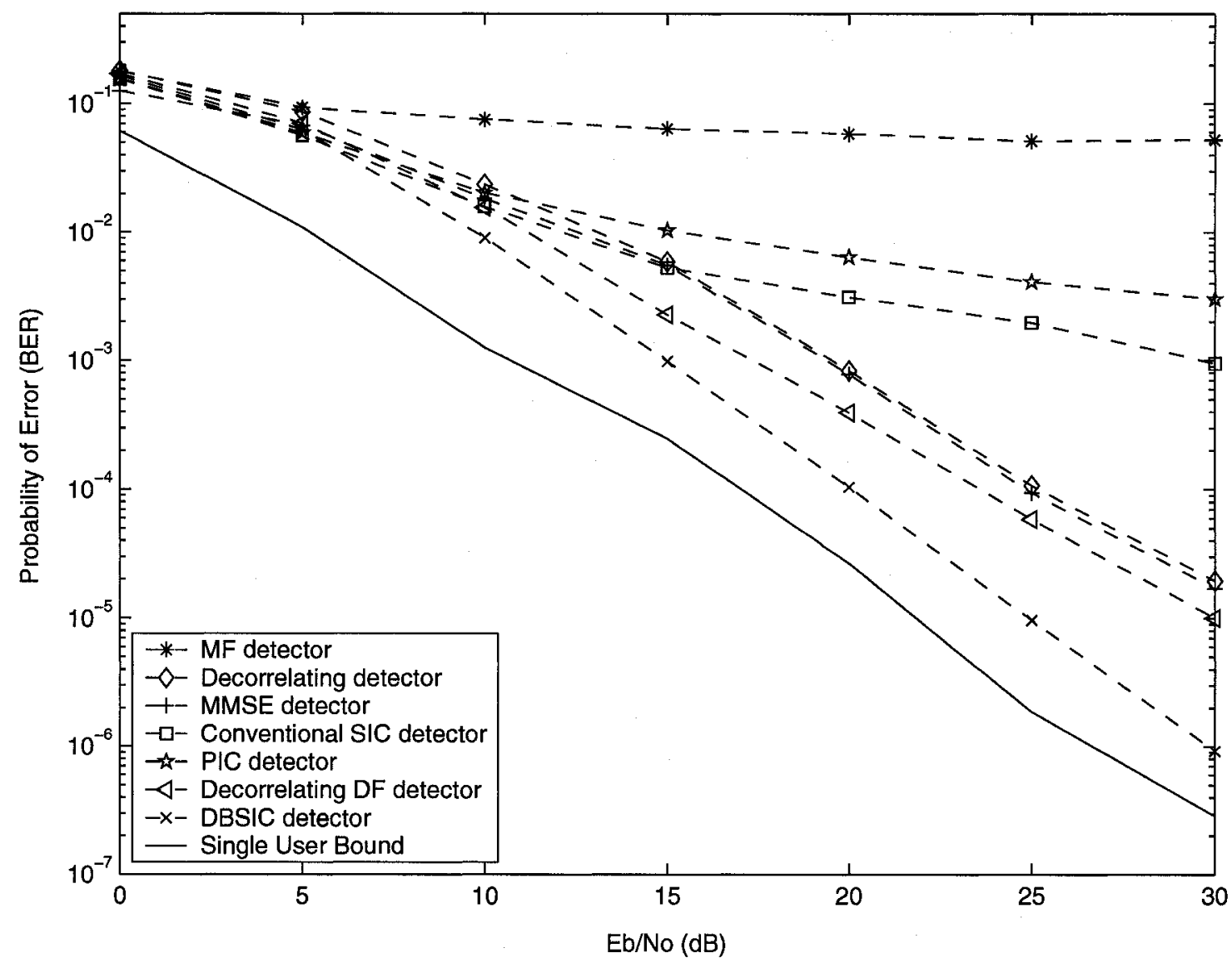

Fig. 6.22 Performance over 2-path Rayleigh fading channel with RAKE, perfect channel estimation, $K_{1}=17, K_{2}=3, G_{1}=64$

\subsubsection{Effect of extremely disparate rates}

Fig 6.23 presents BER performance for a 2-rate system with one user of class 1 and one user of class 30 , corresponding to a case where users have extremely different rates. The processing gain for the lowest rate user is 64. It is shown that DBSIC still outperforms the other receivers in that difficult scenario, with for example a $3.8 \mathrm{~dB}$ gain over the decorrelating DF detector at a BER of $10^{-4}$. 


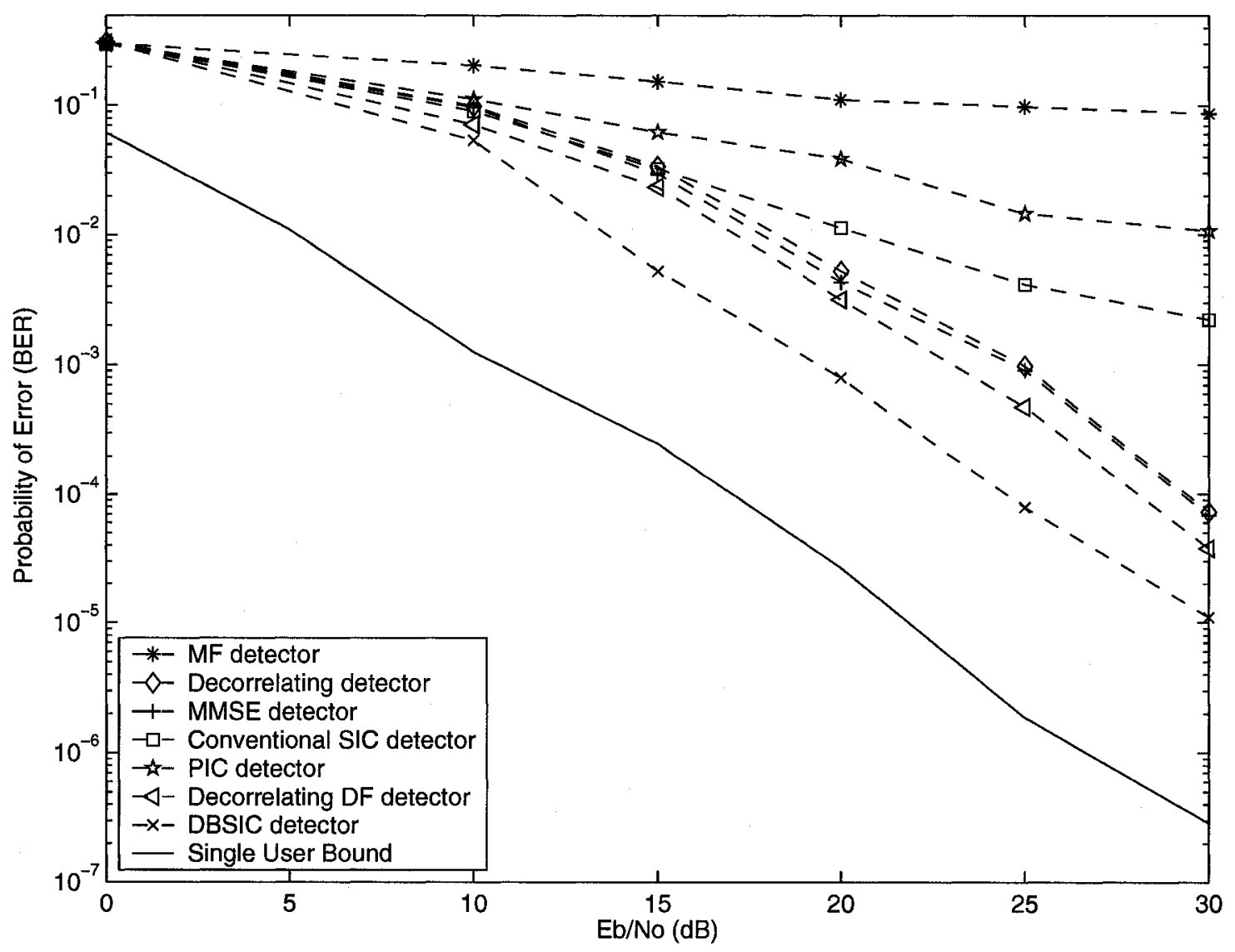

Fig. 6.23 Performance over 2-path Rayleigh fading channel with RAKE, perfect channel estimation, $K_{1}=1, K_{30}=1, G_{1}=64$

The results depicted in Figs. 6.5-6.23 are based on the condition of perfect channel estimation. In other words it is assumed that the true channel information is known to the receiver all the time. Theoretically the channel coefficients can be perfectly tracked/estimated by the receiver which enables the detectors to achieve excellent performance. However, in practical wireless systems, the true channel coefficients are unknown to the receiver, instead the receiver uses estimates of the channel information which are usually imperfect due to the existence of noise and interference. Therefore, the effect of channel estimation errors has to be considered when evaluating 
the performance of a detector scheme, which is studied next.

\subsection{Performance in the case of imperfect channel estimation}

As an example of non-perfect channel estimation, it is assumed that the estimated channel fading coefficient of each path is equal to the true channel coefficient multiplied by a random variable uniformly distributed between $-6.25 \%$ and $6.25 \%$, which is referred in this work as $12.5 \%$ channel estimate error. Case denoted as " $25 \%$ channel estimate error" is defined similarily.

\subsubsection{Effect of having multirate users}

An example of $12.5 \%$ channel estimate error is illustrated in Fig. 6.24. DBSIC performance under perfect channel estimation has also been included as a reference using circle markers. It is clear from Fig. 6.24 that the quality of channel estimates plays a big role in system performance for all multi-user detectors. However, while all receivers considered in this work including DBSIC undergo performance loss under imperfect channel estimation, DBSIC still outperforms the other receivers and achieves for example a $1.5 \mathrm{~dB}$ gain over the decorrelating $\mathrm{DF}$ scheme at a BER of $5 \cdot 10^{-3}$ in a 4-rate system with $12.5 \%$ channel estimate error ${ }^{1}$.

\subsubsection{Effect of user cancellation order}

Both conventional SIC and DBSIC schemes assume that the users are reordered for cancellation based on the outputs of the bank of matched filters. These outputs and hence the ordering/cancellation of users will depend on the transmission signal's power/amplitude, the true channel fading coefficients as well as the estimated channel

\footnotetext{
${ }^{1}$ as defined in the introduction of section 6.5 .
} 


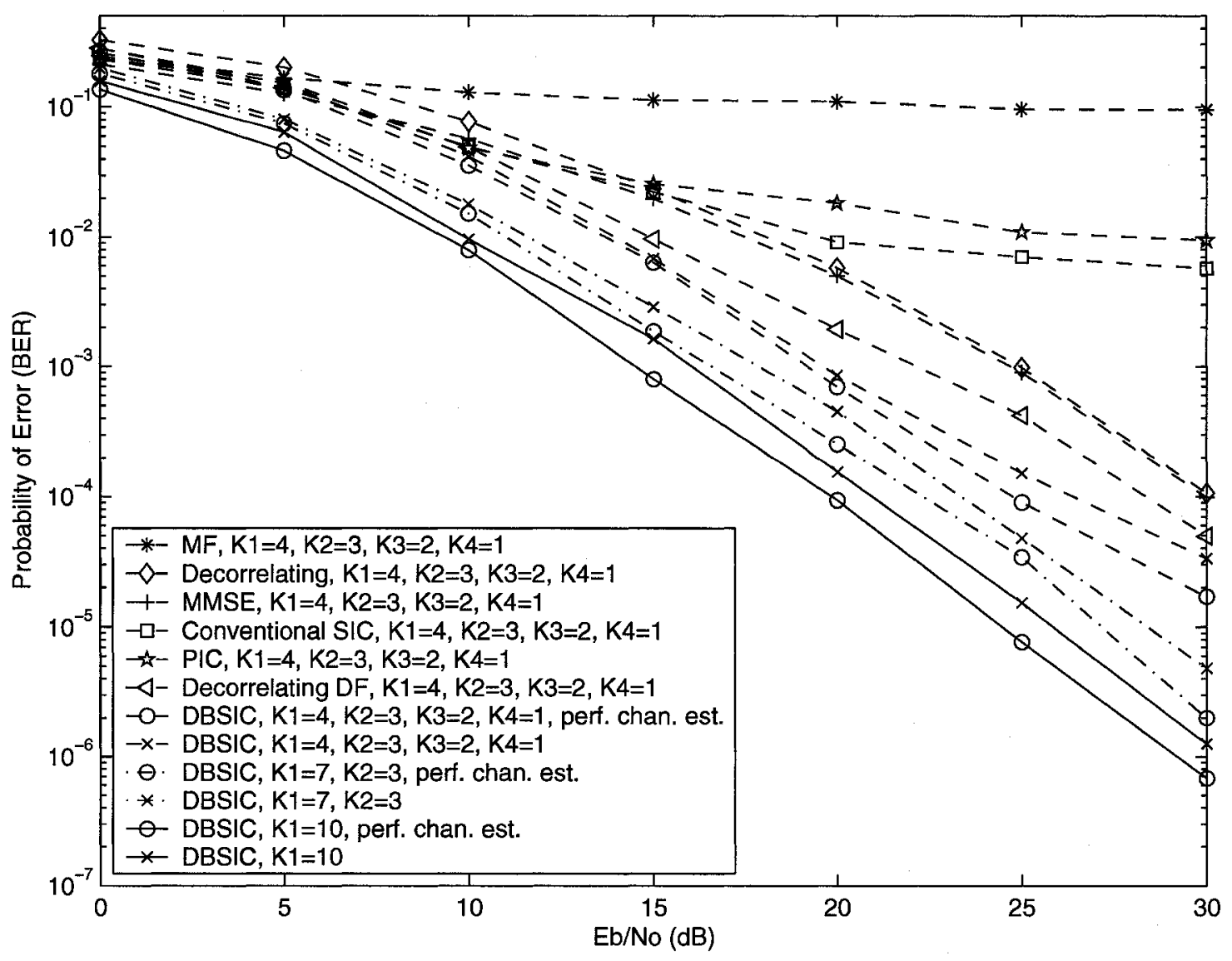

Fig. 6.24 Performance over 2-path Rayleigh fading channel with RAKE, 12.5\% channel estimate error, single and multi-rates, $G_{1}=32$

fading coefficients used by the detectors. Therefore, it is important to assess the effect of imperfect channel estimation on the performance of those receivers when various ordering/cancellation strategies are used.

Similar to section 6.4 .3 , we will consider the conventional case where users are ordered/cancelled based on the outputs of the bank of MF (denoted as 'MF output order') and the case where users are ordered/cancelled randomly (denoted as 'random order'). Fig. 6.25 and Fig. 6.26 present BER performance with $12.5 \%$ and $25 \%$ channel estimate error, respectively. The penalty is approximately $8.6 \mathrm{~dB}$ performance 


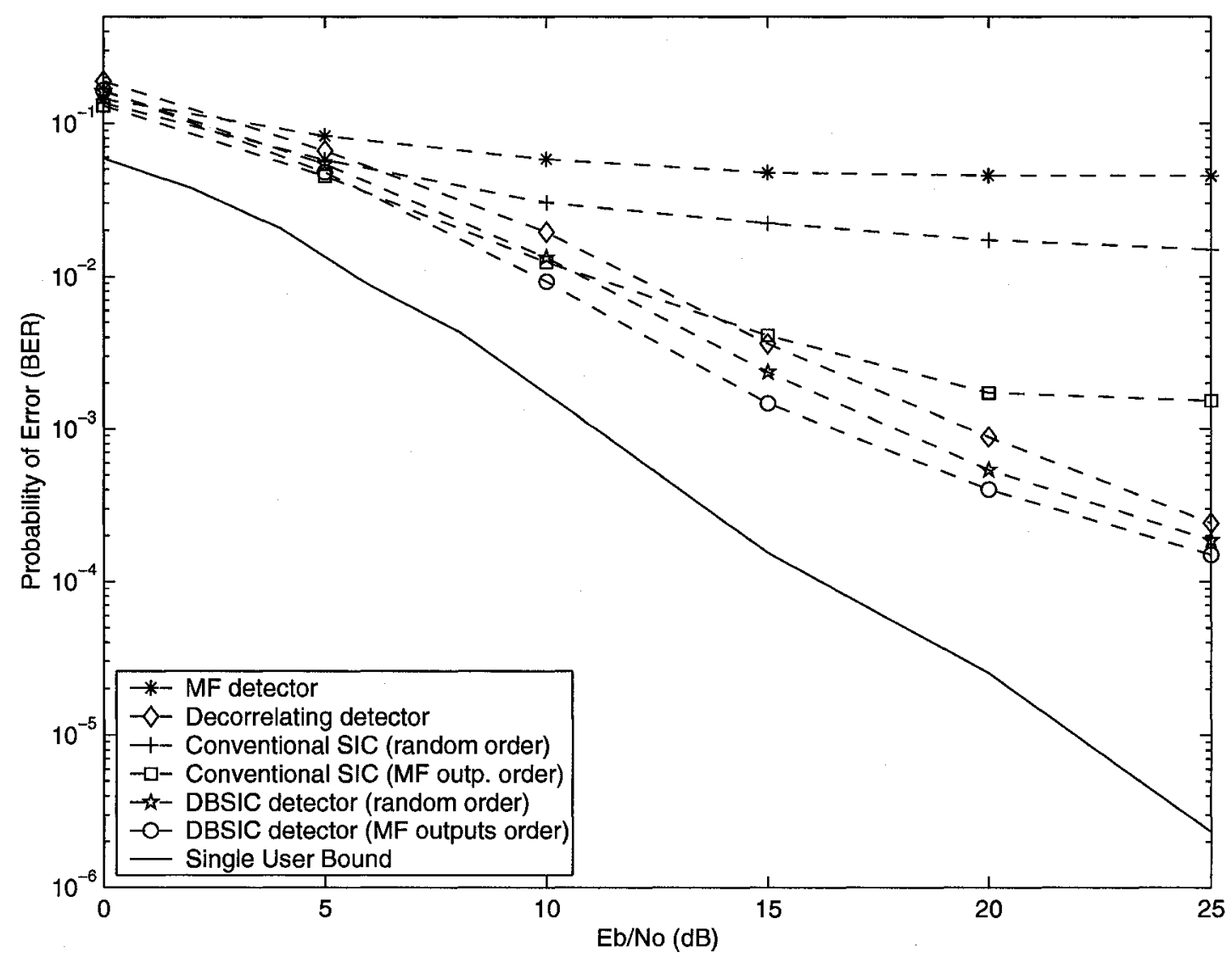

Fig. 6.25 BER performance over 2-path Rayleigh fading channel with RAKE, $12.5 \%$ channel estimate error, $K_{1}=3, K_{2}=2, G_{1}=32$ (MF outputs $/$ random user cancellation order)

degradation for DBSIC detector compared to Rake receiver single-user bound when the channel estimate error is $12.5 \%$. The loss of performance is even bigger when estimate error increases to $25 \%$. However, in the case of $12.5 \%$ channel estimate error, DBSIC still outperforms the decorrelating detector by $2.5 \mathrm{~dB}$ when its user cancellation/detecting order is based on the MF outputs and by $1 \mathrm{~dB}$ when its user cancellation/detecting order is random at a BER of $3 \cdot 10^{-4}$. Fig. 6.25 also shows that DBSIC outperforms the conventional SIC when both have user cancellation/detecting 


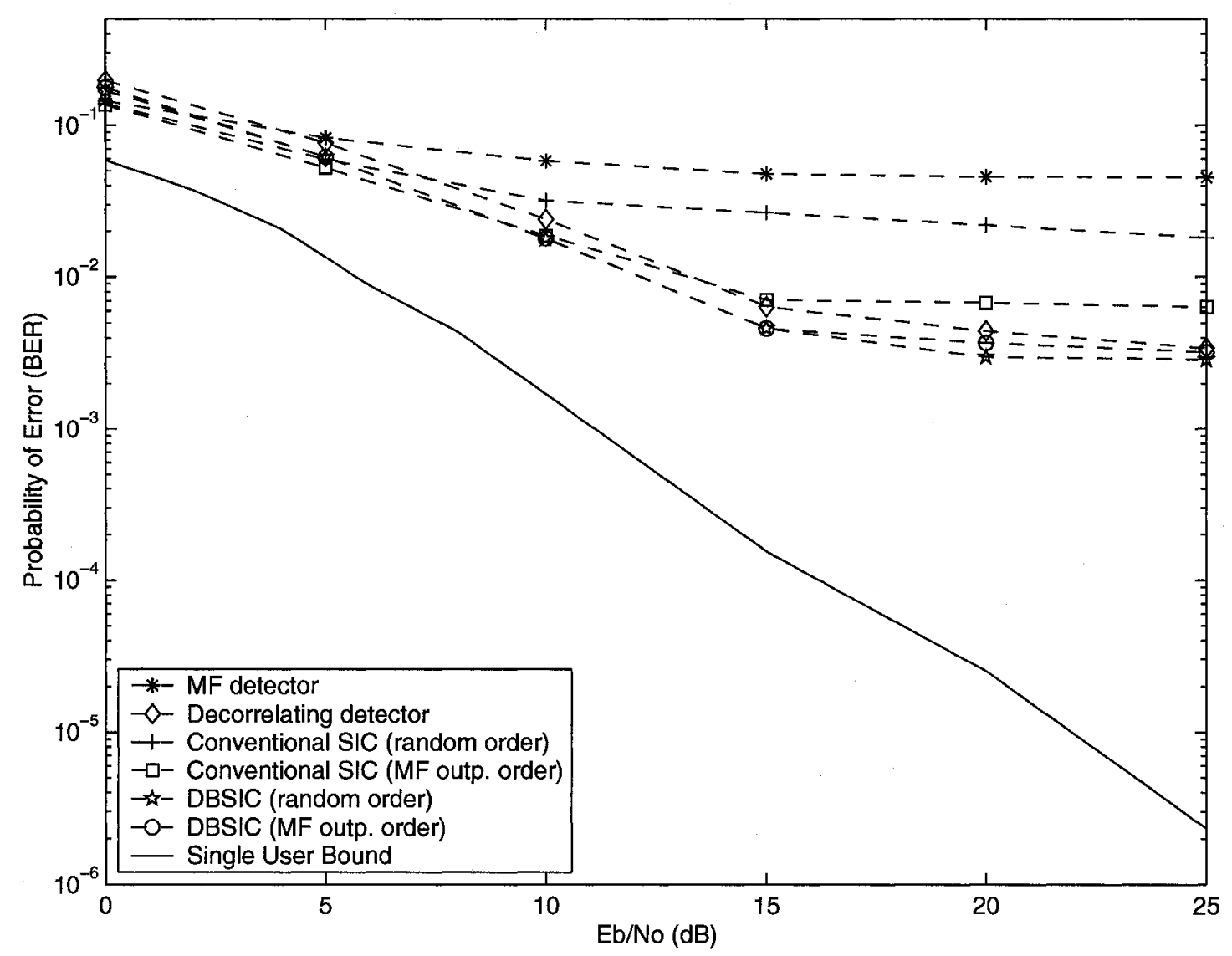

Fig. 6.26 BER performance over 2-path Rayleigh fading channel with RAKE, $25 \%$ channel estimate error, $K_{1}=3, K_{2}=2, G_{1}=32$ (MF outputs/random user cancellation order)

order based on MF outputs. It is also seen that DBSIC with random detecting order outperforms significantly the conventional SIC with random detecting order under $12.5 \%$ channel estimate error, showing that DBSIC performance is much less sensitive to the user cancellation order than the conventional SIC also under imperfect channel estimation.

Figs.6.27-6.29 compare the BER performance of each receiver for various degrees of channel estimate error. We observe from Figs.6.27-6.29 that with the increase of 


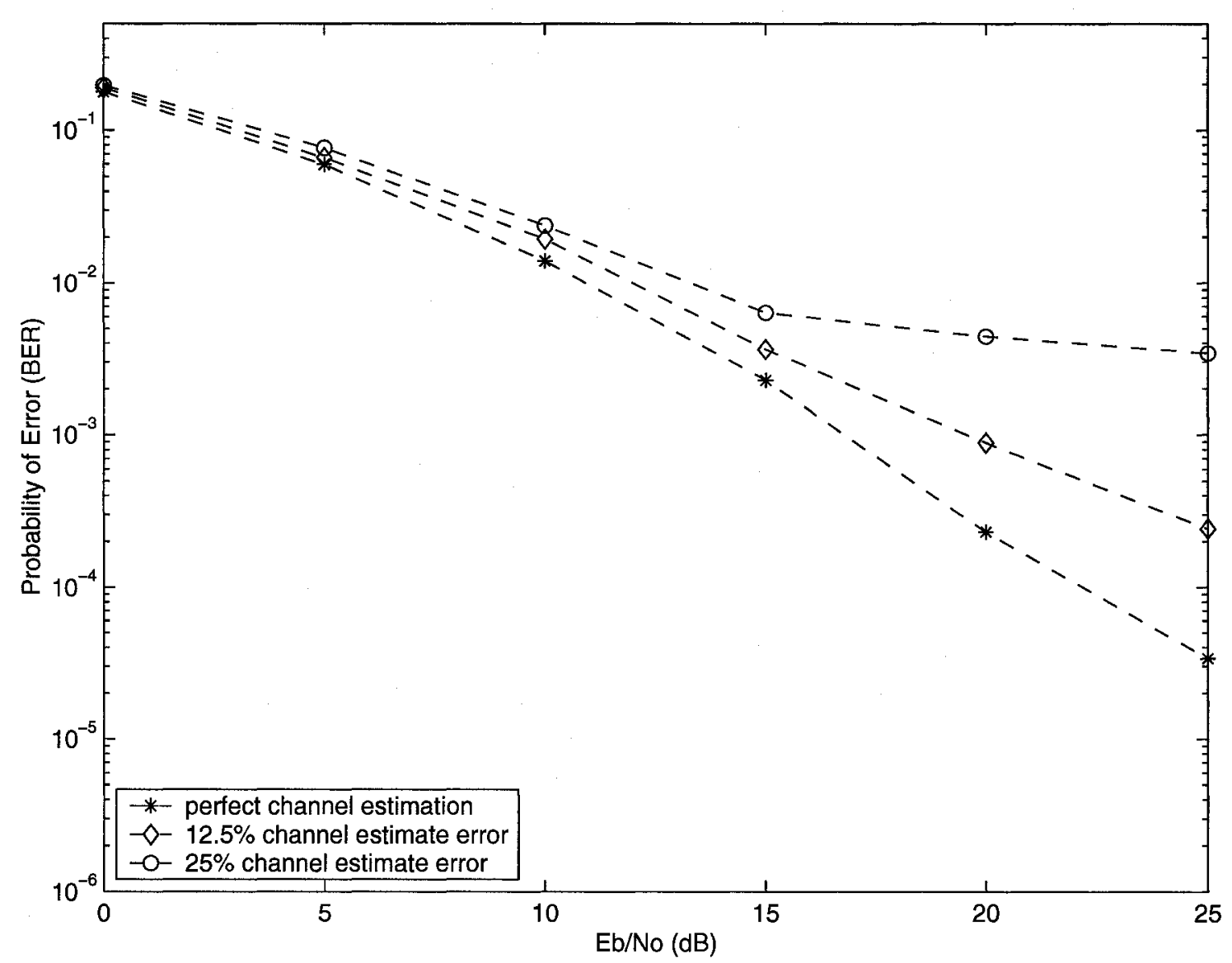

Fig. 6.27 BER performance over 2-path Rayleigh Fading channel with RAKE, Decorrelating detector, $K_{1}=3, K_{2}=2, G_{1}=32$

channel estimate errors (for example $25 \%$ channel estimate error), the performance for all the detectors improves little when $E_{b} / N_{0}$ increases from $15 \mathrm{~dB}$ to $25 \mathrm{~dB}$. This is because channel estimation errors cancel out the performance gains obtained by the multi-user detector schemes. Therefore, it is seen that to benefit from advanced multiuser detection techniques such as DBSIC or even of commonly used multiuser schemes, a channel estimator with reasonably good capabilities must be used so that channel estimation errors are under control and within reasonable bounds. One approach to address this problem consists of designing joint channel estimation and 
multiuser detection schemes and one such design has been proposed in chapter 4 . Its performance in terms of channel estimate MSE and BER will be presented next.

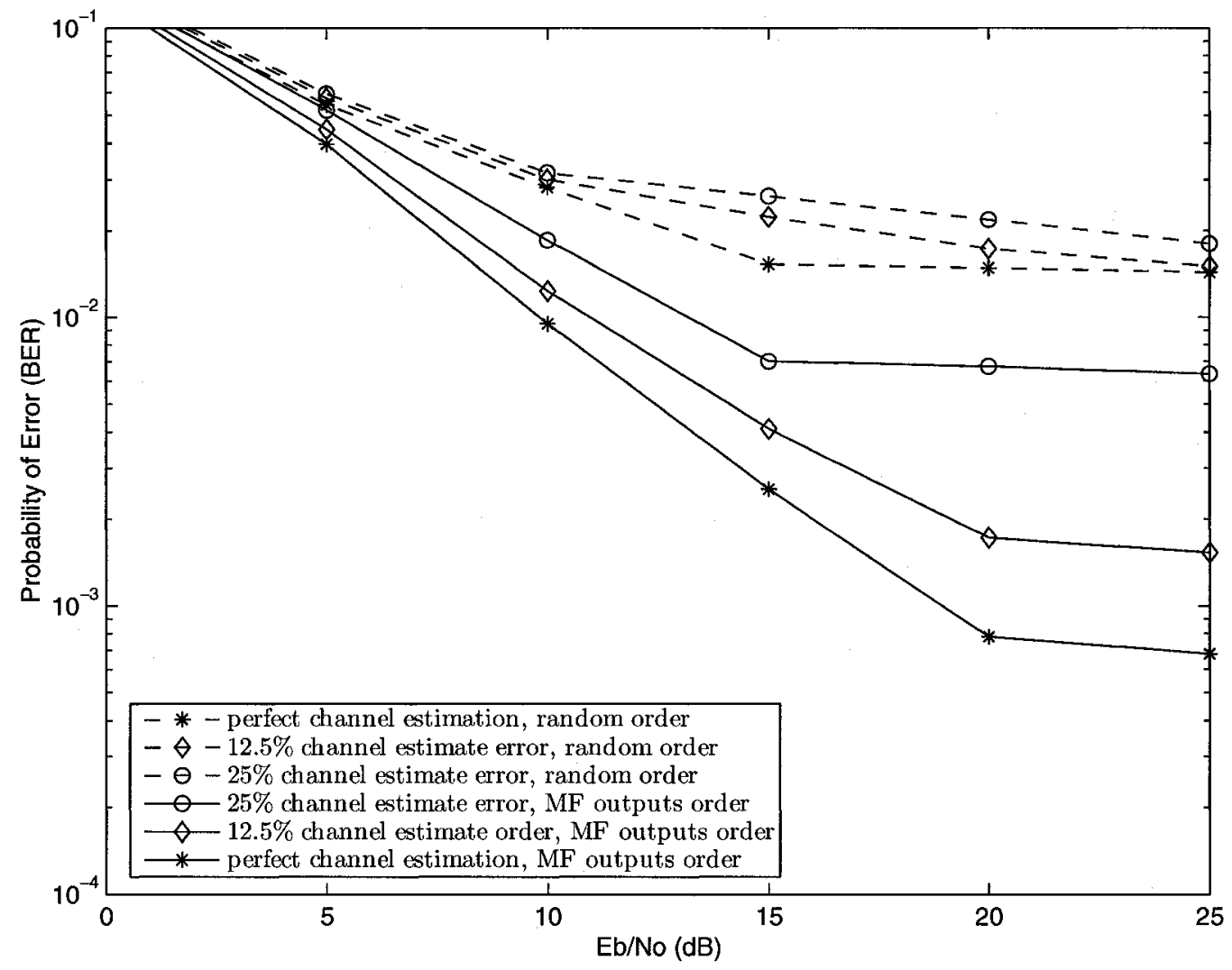

Fig. 6.28 BER performance over 2-path Rayleigh Fading channel with RAKE, conventional SIC detector (MF outputs/random user cancellation ordering), $K_{1}=$ $3, K_{2}=2, G_{1}=32$ 


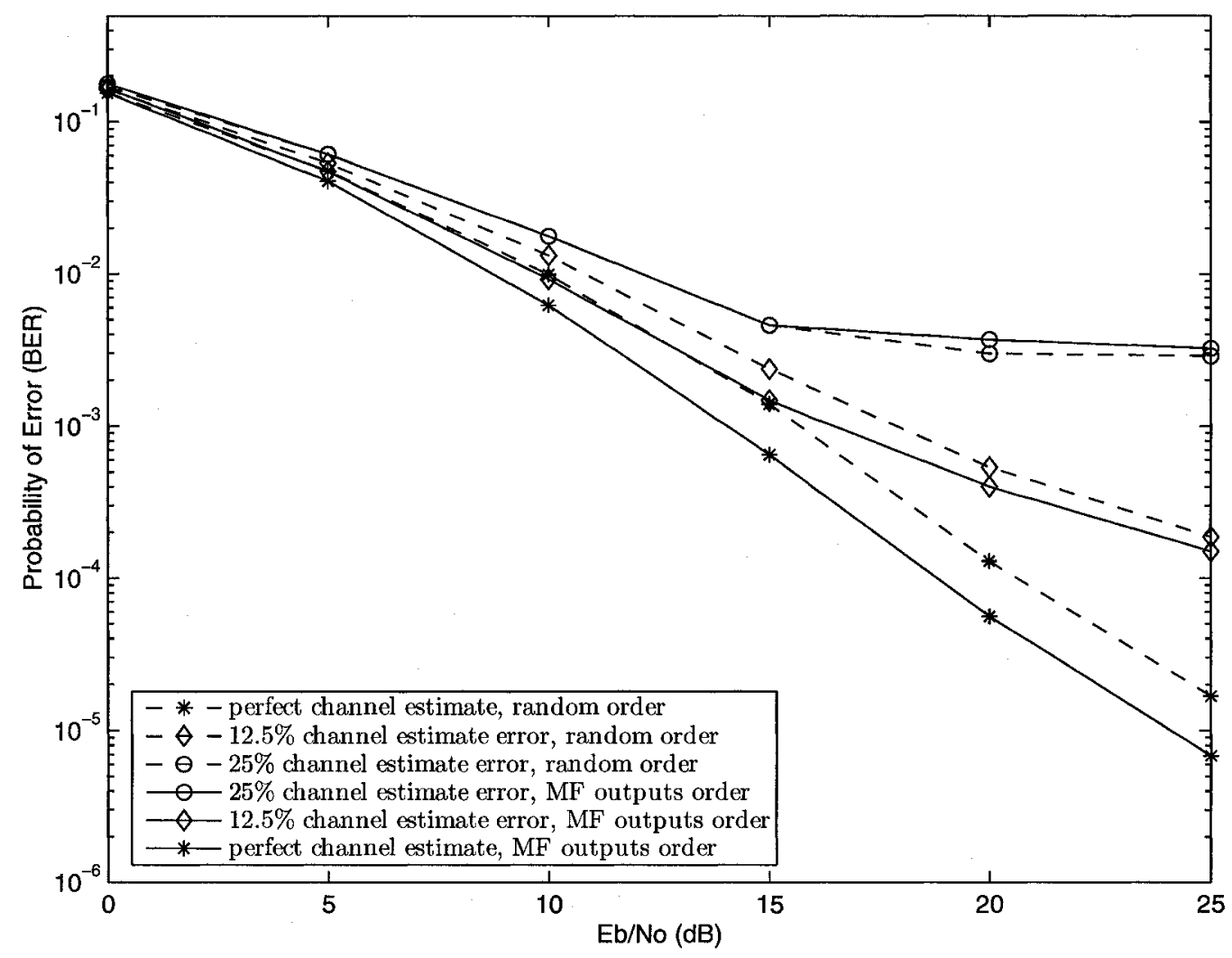

Fig. 6.29 BER performance over 2-path Rayleigh Fading channel with RAKE, DBSIC detector (MF outputs/random ordering), $K_{1}=3, K_{2}=2, G_{1}=32$

\subsection{Performance of the joint channel estimator multiuser detector}

\subsubsection{Performance of the channel estimator}

The effectiveness of the channel estimator proposed in section 4.2 is first demonstrated in terms of the normalized MSE of the channel fading gains of the virtual users. We define the normalized MSE as $E\left[\left\{\vec{\alpha}_{v}-\hat{\vec{\alpha}}_{v}\right\}^{H}\left\{\vec{\alpha}_{v}-\hat{\vec{\alpha}}_{v}\right\}\right] / E\left[\vec{\alpha}_{v}^{H} \vec{\alpha}_{v}\right]$, where the elements of $\hat{\vec{\alpha}}_{v}$ (see (4.11)) are obtained from (4.14). 
Fig. 6.30 shows the normalized MSE of the channel fading coefficients of the virtual users for a system with ten physical users having same or different rates as indicated in Table 6.1. We assume that the joint channel estimator multiuser detector performs one iteration (e.g., step1-step4 from section 4.2) and the MSE is calculated after step 3. In Step 1, 3 training symbols are considered. Then in step 2, 15 symbols are sent through the channel and detected using DBSIC.

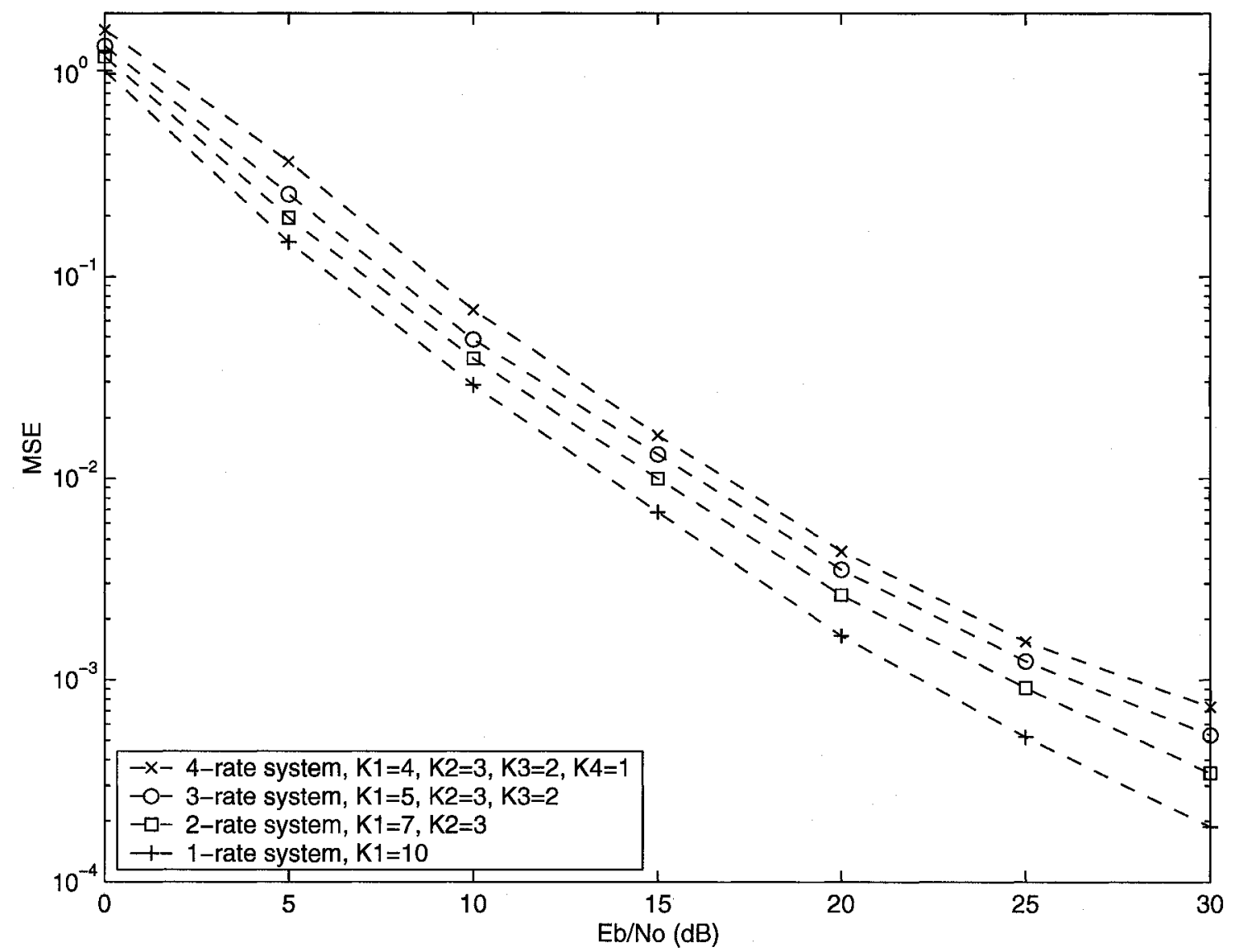

Fig. 6.30 Normalized MSE of channel fading coefficients of the virtual users over 2-path Rayleigh fading channel with RAKE, $G_{1}=32$

It is seen from Fig. 6.30 that in general the proposed channel estimator achieves good performance especially in the scenario of high SNR. The performance deterio- 
rates when the SNR becomes smaller or the system load becomes heavier (e.g. when some users have higher rates).

\subsubsection{Performance of the joint channel estimator multiuser detector}

Figs. 6.31-6.34 show the BER performance of the joint channel estimation multiuser detection scheme illustrated in Fig. 4.1 when either DBSIC or other detectors

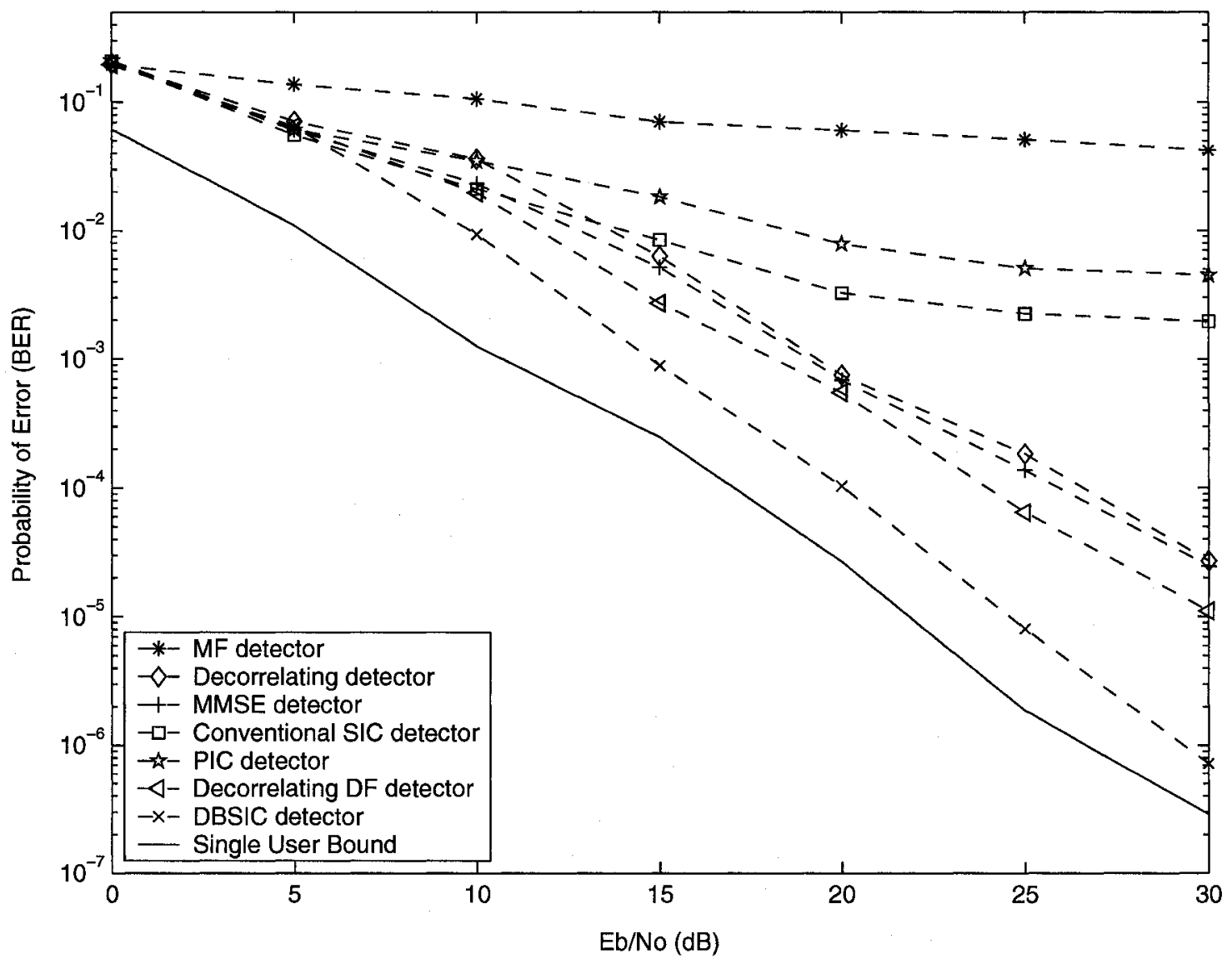

Fig. 6.31 Performance over 2-path Rayleigh fading channel with RAKE, joint channel estimation and multiuser detection, $K_{1}=10, G_{1}=32$ 
are used for the multiuser detection part of the receiver. A ten user single-rate and multirate CDMA system is considered according to the cases outlined in Table 6.1. One iteration in the receiver is assumed (e.g., step1-step4 are performed once).

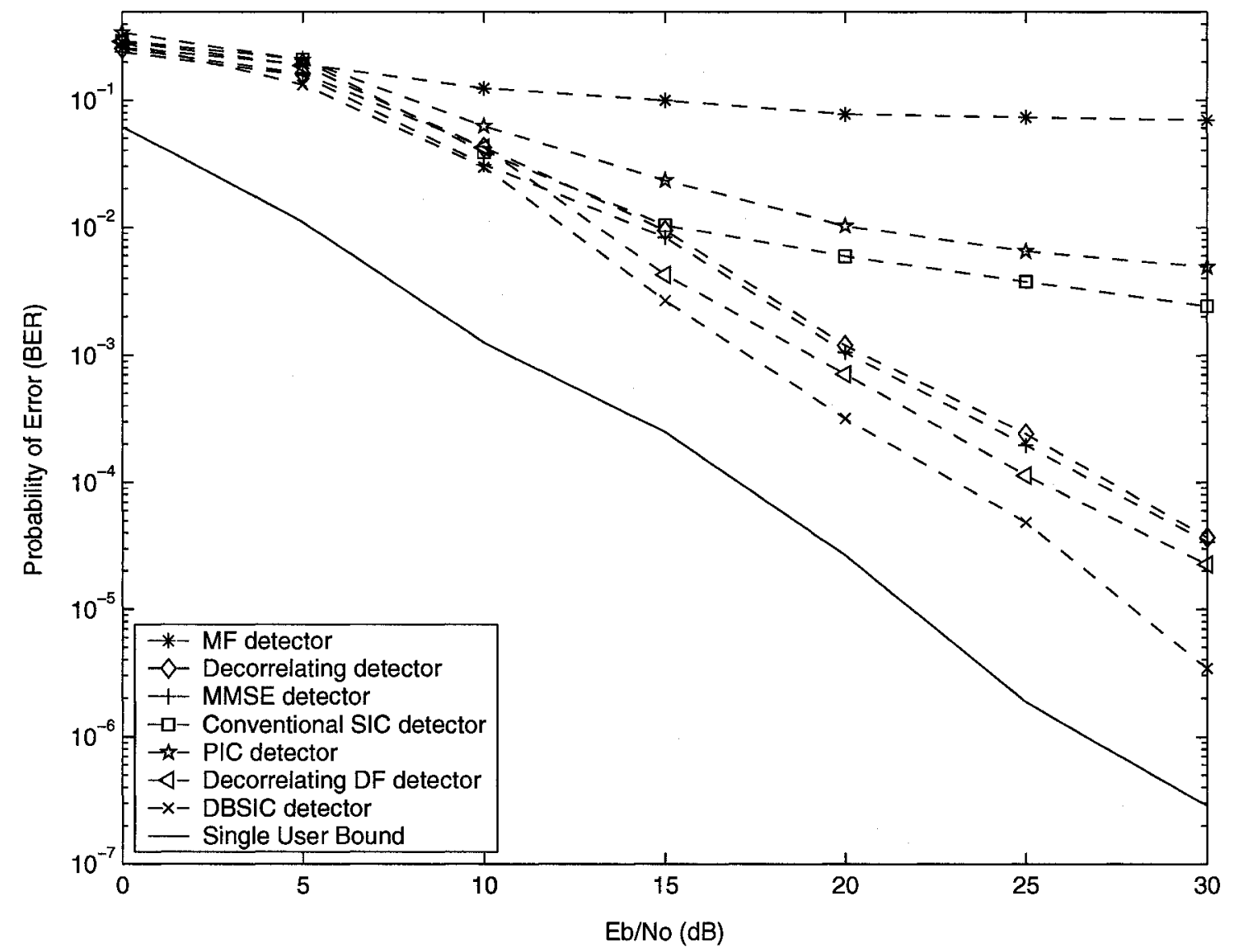

Fig. 6.32 Performance over 2-path Rayleigh fading channel with RAKE, joint channel estimation and multiuser detection, $K_{1}=7, K_{2}=3, G_{1}=32$

It can be seen from Figs. 6.31-6.34 that the joint channel estimator multiuser detector scheme that employs DBSIC in its detection part outperforms the other joint channel estimator multiuser detectors that employ other types of multiuser detection techniques. Although the performance is degraded as the number of multirate users and the rates of the users increases (heavier system load for the virtual users), 


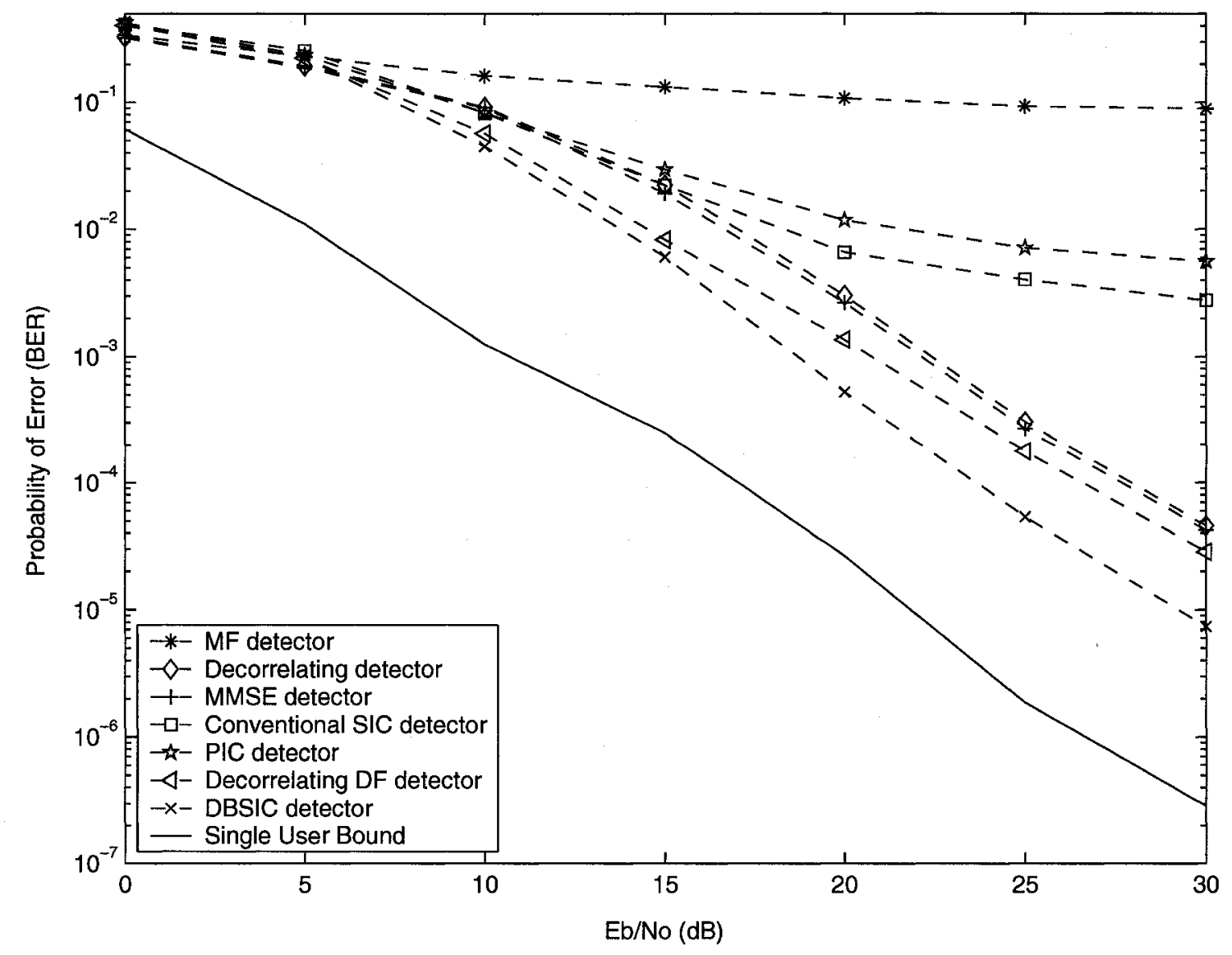

Fig. 6.33 Performance over 2-path Rayleigh fading channel with RAKE, joint channel estimate and multiuser detection, $K_{1}=5, K_{2}=3, K_{3}=2, G_{1}=32$

Figs. 6.31-6.34 show that the DBSIC based joint channel estimator multiuser detector is still the best. 


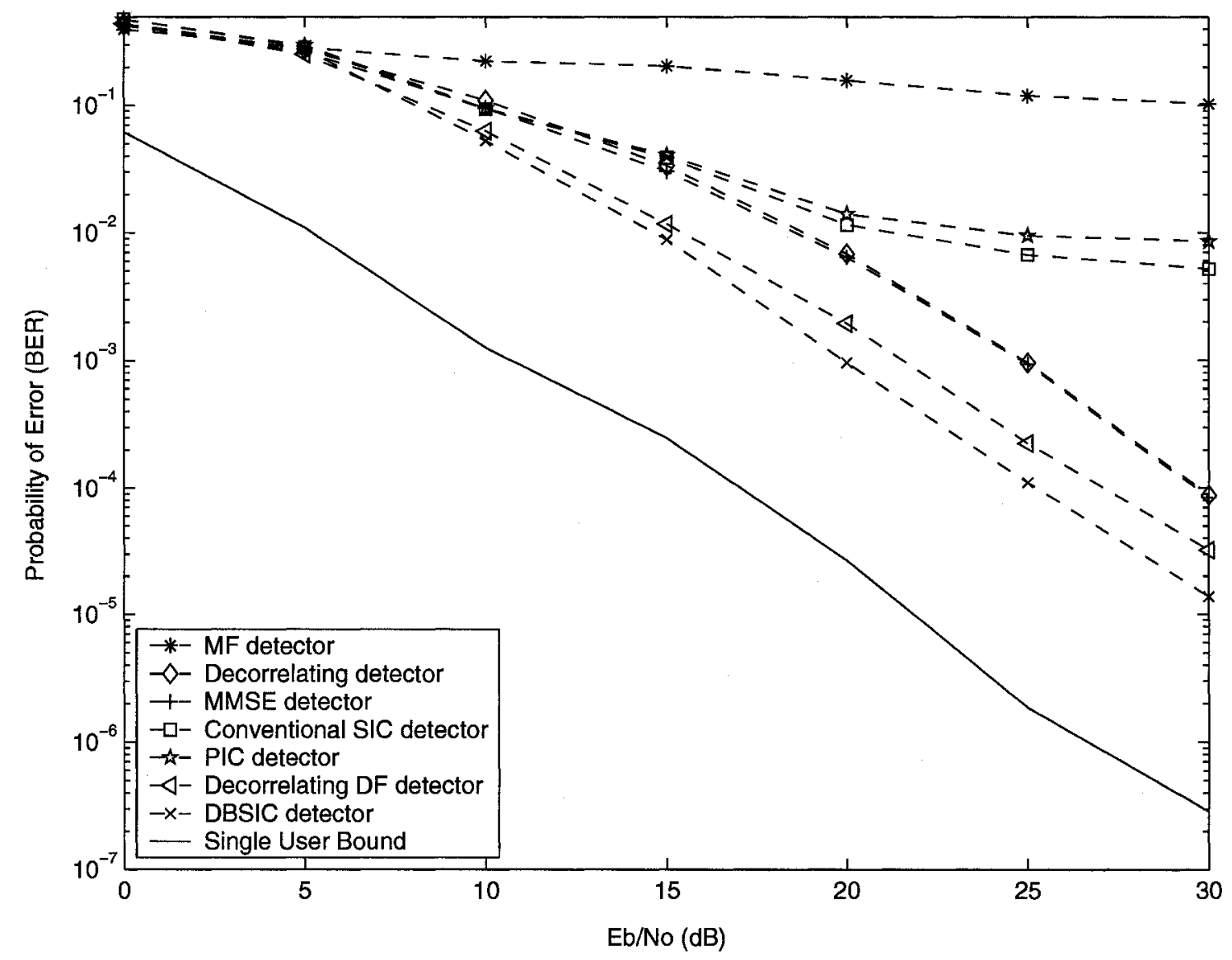

Fig. 6.34 Performance over 2-path Rayleigh fading channel with RAKE, joint channel estimate and multiuser detection, $K_{1}=4, K_{2}=3, K_{3}=2, K_{4}=1$, $G_{1}=32$

Fig. 6.35 shows the BER performance of the joint DBSIC-based channel estimation and multiuser detection scheme and DBSIC assuming perfect channel estimation for 10 users with same or different rates. It can be seen that although the performance of DBSIC is degraded by the channel estimation process, the differences are not significant at a sufficiently high SNR. For example, in a single rate case, the joint channel estimator multiuser detector based on DBSIC performs almost the same as DBSIC with perfect channel estimation. In a 2-rate case, the performance of DBSIC in the system employing this channel estimator is close to the scenario of perfect channel es- 


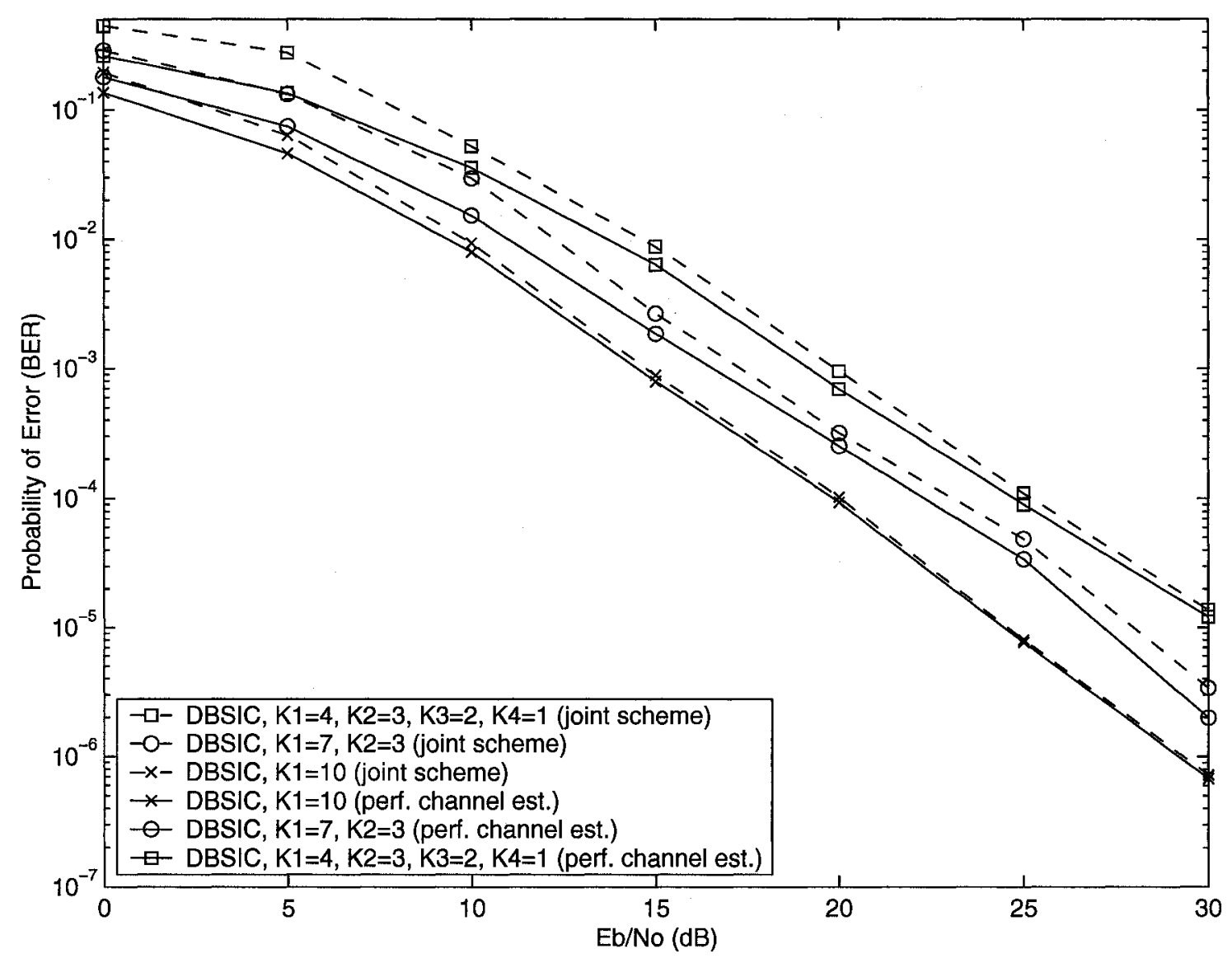

Fig. 6.35 Performance of DBSIC over 2-path Rayleigh fading channel with RAKE, (joint channel estimation and multiuser detection versus multiuser detection with perfect channel estimation), single and multi-rate, $G_{1}=32$

timation. For a given SNR, it is observed that larger degradation is observed for cases with a larger number of high rate users. This is to be expected as the rates of users increases, VPG implies a higher system load in terms of virtual users, hence MAI is more significant. For low SNR, there is a difference between the performance of the joint DBSIC-based scheme and DBSIC with perfect channel estimation. This is to be expected as channel fading estimates obtained from the training sequences are subject to larger errors due to the higher level of noise and larger errors are also expected in the detection of the subsequent symbols to be used later as training. Therefore, even 
the new channel estimates after the iteration will be subject to channel estimation error reducing the overall system performance compared to perfect channel estimation case.

Figs. 6.36-6.39 compare the BER performance of the joint scheme with various multiuser detectors used in the detection part with their counterparts under perfect channel estimation.

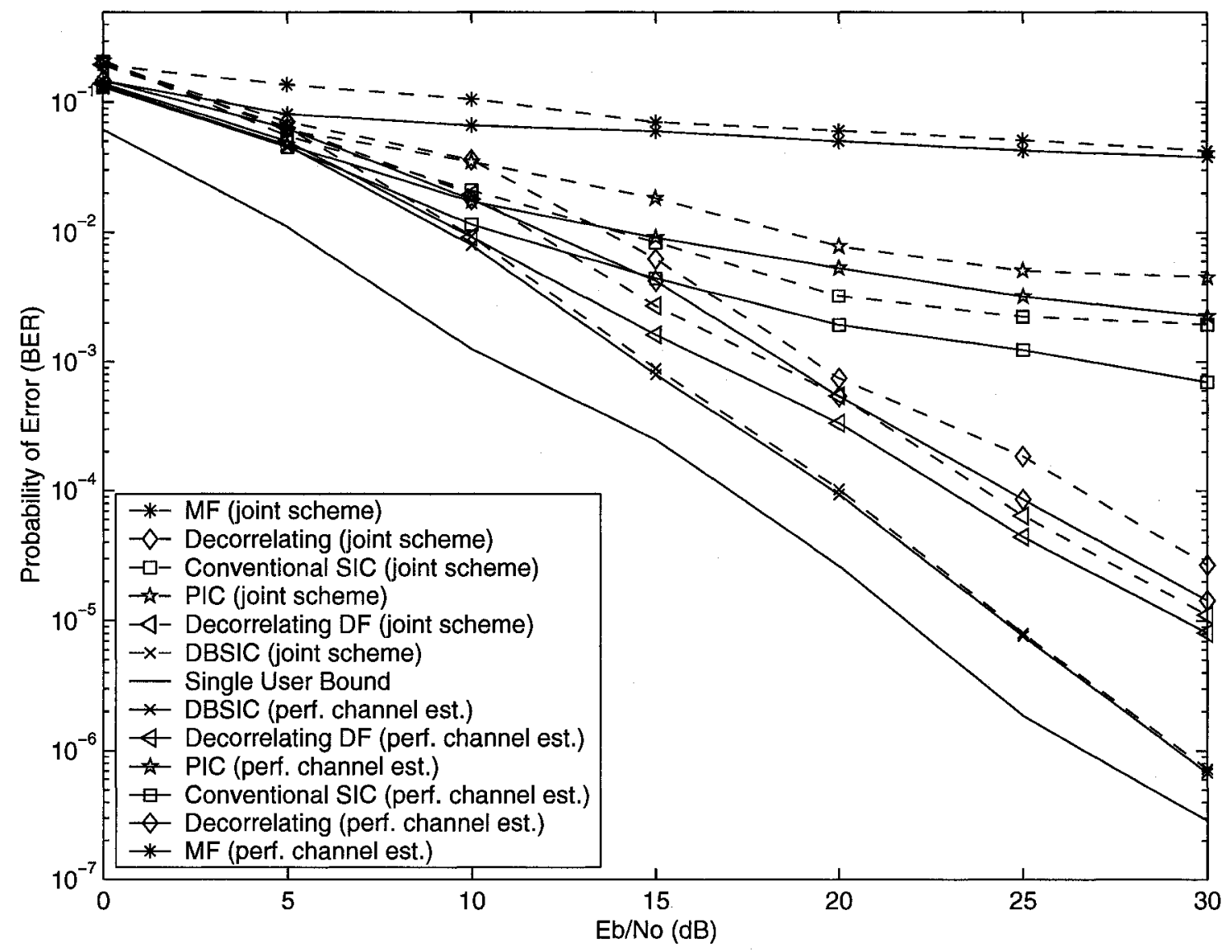

Fig. 6.36 Performance over 2-path Rayleigh fading channel with RAKE, (joint channel estimation and multiuser detection versus multiuser detection with perfect channel estimation), $K_{1}=10, G_{1}=32$

It can be seen from Figs. 6.36-6.39 that the performance of all detectors is degraded by the channel estimation process, and more importantly that the degradation in 


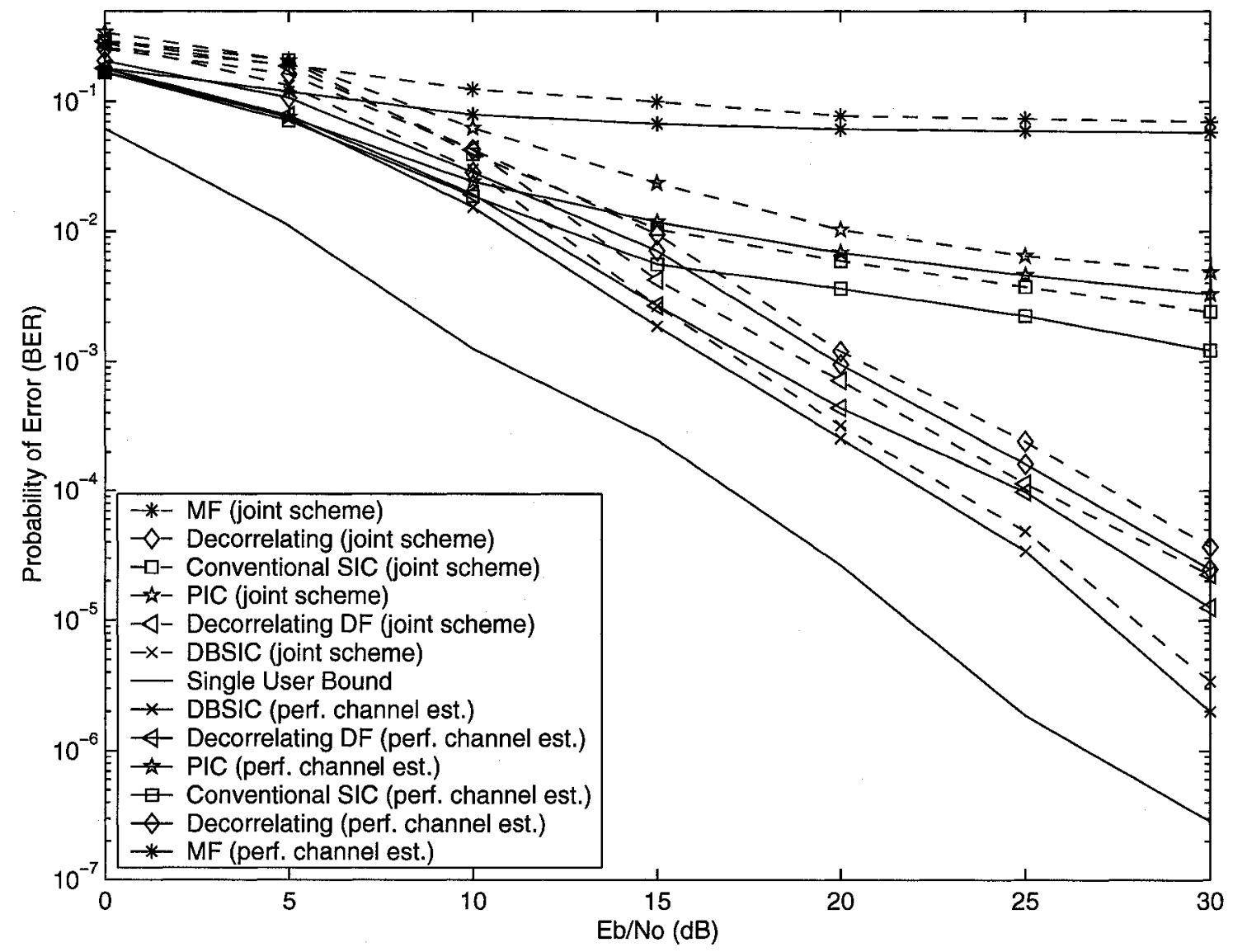

Fig. 6.37 Performance over 2-path Rayleigh fading channel with RAKE, (joint channel estimation and multiuser detection versus multiuser detection with perfect channel estimation), $K_{1}=7, K_{2}=3, G_{1}=32$

performance compared to perfect channel estimation is smaller for the joint DBSICbased scheme compared to the other joint detectors.

All the simulations presented in this chapter confirm the superiority of DBSIC under various scenarios, either considered by itself or combined with a channel estimator into an iterative joint channel estimator multiuser detector. It is to be noted that for simplicity an uncoded DS-CDMA system with no filtering was assumed. Practical implementation of DBSIC would require modification of its structure to take into account more practical pulse shapes, for example of raised-cosine types. Furthermore, 


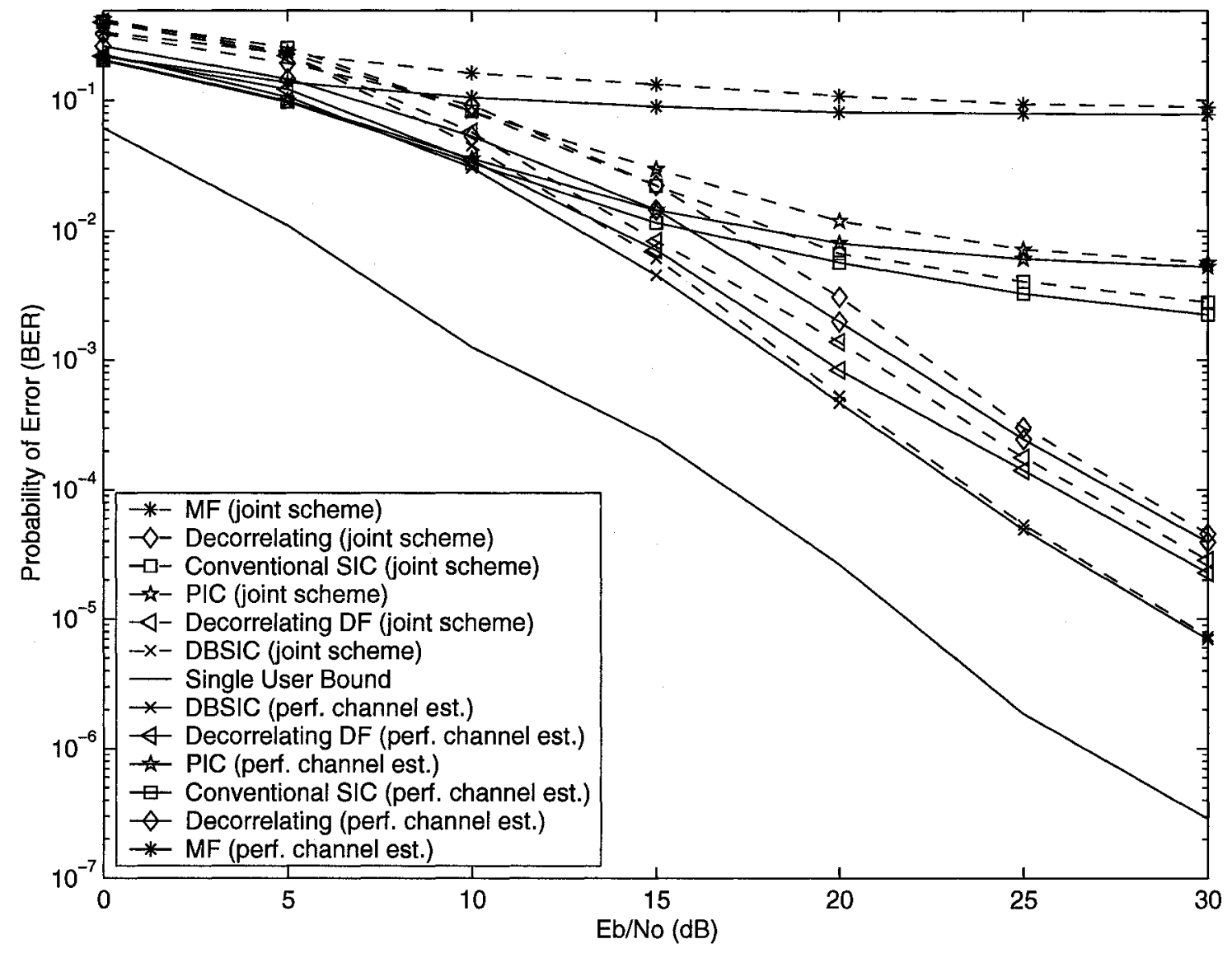

Fig. 6.38 Performance over 2-path Rayleigh fading channel with RAKE, (joint channel estimation and multiuser detection versus multiuser detection with perfect channel estimation), $K_{1}=5, K_{2}=3, K_{3}=2, G_{1}=32$

in a practical system, channel coding would be implemented on top of the transceiver. Therefore, the target (coded) BER for practical comparisons before channel decoding is more likely to be in the range of 5 to $15 \%$. A coded BER of 5 to $15 \%$ (or $20 \%$ for sophisticated schemes such as turbo codes) may translate into a BER of $10^{-3}$ to $10^{-6}$ after channel decoding. Therefore, DBSIC gains implemented in a practical system will probably be smaller than the ones outlined in this chapter. 


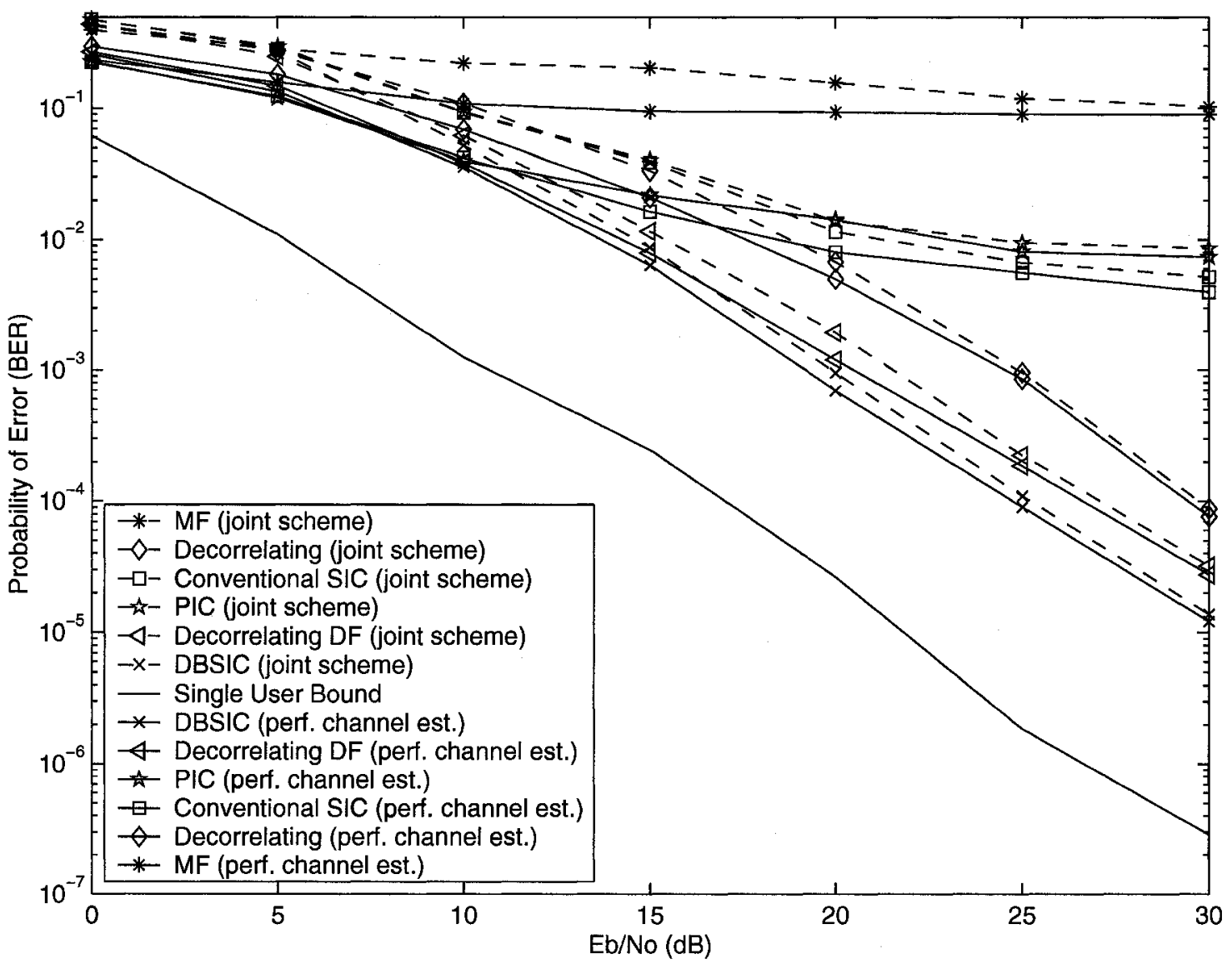

Fig. 6.39 Performance over 2-path Rayleigh fading channel with RAKE, (joint channel estimation and multiuser detection versus multiuser detection with perfect channel estimation), $K_{1}=4, K_{2}=3, K_{3}=2, K_{4}=1, G_{1}=32$ 


\section{Chapter 7}

\section{Conclusions and Future Work}

\subsection{Conclusion and summary}

This work investigates multirate multiuser DS-CDMA detection techniques for multipath fading channels. SIC and decorrelating detectors are two suboptimum multiuser detectors employed in CDMA systems to combat the MAI, ISI and near-far effects. Since SIC uses estimates of previous users'signals in the interference cancellation used to detect the next user, it might be subject to performance degradation due to error propagation in case of poor MAI estimate. To improve the MAI estimate, the novel Decorrelator-Based Successive Interference Cancellation (DBSIC) scheme for asynchronous VPG multirate CDMA systems is proposed. Unlike the conventional SIC, the DBSIC scheme implements a decorrelator on top of the conventional matched filtering to detect the user's signal that will be used at the next interference cancellation stage. Due to the fact that the decorrelating detector outperforms the conventional MF detector particularly at high SNR and the SIC detector usually starts the detection from the users with the strongest receiving power or signal amplitude, DBSIC can have a much more accurate signal estimate at each stage than the conventional 
SIC which is crucial for the subsequent stages at the expense of reasonable additional complexity. A multirate DS-CDMA system simulator is developed. The performance of DBSIC is assessed in terms of asymptotic multiuser efficiency (AME) and bit error rate (BER) obtained using an analytical approach and simulations.

The analytical AME performance analysis for DBSIC is derived for AWGN and Rayleigh fading channels. The results are compared with that of conventional singleuser MF, optimum, decorrelating and SIC receivers. In particular, it is shown that although DBSIC has a lower AME than the optimum receiver for AWGN channels when the interfering user is weaker than the user of interest, its AME approaches the unity optimum receiver AME as the interferer becomes stronger than the user of interest. For all values of the spreading codes correlation considered, DBSIC shows a similar AME curve shape as the conventional SIC receiver but has a higher AME which remains positive for all user energy ratios, showing that DBSIC is near-far resistant. It is shown that DBSIC is completely near-far resistant over Rayleigh fading channels with the same AME as the decorrelating scheme for user 1 and as the optimum scheme for user 2, both AMEs being independent of the user energy ratio.

DBSIC performance is also assessed in terms of BER using an analytical analysis based on a Gaussian approximation and using simulations. A good match between simulation and the approximate theoretical curves is observed. DBSIC improvement over commonly used multiuser detection schemes such the decorrelating, MMSE, SIC, PIC and decorrelating DF detectors is demonstrated over frequency-selective Rayleigh multipath fading channels with both perfect and imperfect channel side information and different number of data rate classes (including cases with more than 2 rates). In particular, DBSIC provides gains even in the scenario of heavy virtual system load when users' data rates increase. This thesis also considers the effect of cancellation/detection order on the performance of SIC and DBSIC. It is shown that while 
conventional detecting order based on matched filter outputs outperforms random detecting order for both SIC and DBSIC under perfect or mild imperfect channel estimation, DBSIC performance is less sensitive to the detection ordering method than SIC. It is also demonstrated that the choice of Gold sequences and the method used to generate the signature of the users of higher rate has no impact on the performance gain achieved by DBSIC over the other detectors.

This thesis also proposes a multiuser detection technique which is based on the same principles of multistage PIC but includes the latest decision feedback updates with special rules. An asynchronous decorrelating multiuser detector implementing the ideal noise whitening filter at a low complexity level is also presented. This structure involves a novel adaptive algorithm that factorizes the cross-correlation matrix of the unsynchronized user signature waveforms.

To combat effectively the effects of multipath propagation and MAI, most multiuser detectors require some channel state information (CSI), which needs to be estimated in practice. The performance of wireless communication systems depends mainly on their ability to obtain an accurate estimation of the CSI they require. Channel estimation scheme is studied and an iterative joint channel estimation and DBSIC-based multiuser detection receiver is developed for asynchronous multirate CDMA systems. The effectiveness of this joint channel estimator multiuser detector is supported by results in terms of channel estimates MSE as well as BER performance. It is shown that the joint channel estimator multiuser detector scheme that employs DBSIC in its detection part outperforms the other joint channel estimator multiuser detectors that employ commonly used multiuser detection techniques. It is also shown the degradation in performance compared to perfect channel estimation is smaller for the joint DBSIC-based scheme compared to the other joint detectors. 


\subsection{Future work}

Numerous multiuser detection techniques have been developed to improve the detection performance and increase the capacity and coverage for DS-CDMA systems. First, basic multiuser receivers such as the decorrelating ${ }^{1}$, conventional SIC, PIC, MMSE and decorrelating DF schemes have been proposed, and were shown to yield improved performance compared to the conventional MF detector. Later, hybrid multiuser detection techniques that combined two or more "basic" MUD principles were developed. This thesis considered a few of such structures focusing mainly on DBSIC, a scheme that combines SIC and decorrelating MUD principles, and comparing its performance to basic MUD. Future work could involve a more exhaustive comparison study of several hybrid MUD in terms of performance and complexity. In particular, performance analysis of the proposed modified PIC and the decorrelating detector with reduced complexity proposed in section 3.6 and section 3.7 , respectively could be evaluated.

Generally, new proposed hybrid MUD structures offer superior performance compared to well-known MUD detectors, but complexity is not to be neglected. DBSIC falls into that category achieving much better performance but at the expense of computational complexity due to the presence of matrix inversions. Looking at current CDMA systems, it is seen that CDMA industry is reluctant to implement multiuser detectors that are too complicated even though they offer superior performance ${ }^{2}$. Therefore, finding ways to reduce the complexity of DBSIC is a logical continuation of this work. Section 3.6 which presents an algorithm to implement the decorrelating detector with reduced complexity could be considered as preliminary work towards that goal since both DBSIC and the decorrelating detector perform similar matrix

\footnotetext{
${ }^{1}$ Note that the decorrelating detector outperforms the conventional MF detector at high SNR.

${ }^{2}$ For example, the conventional MF and now recently PIC are mainly implemented.
} 
inversions.

In this thesis DBSIC principles have been presented for rectangular transmit pulse shapes, practical implementation of DBSIC would require modification of its structure to take into account more practical pulse shapes, for example of raised-cosine types. Also, transmission is assumed to be chip synchronous, which might be difficult to achieve in the uplink. Future work could involve presenting simulations of DBSIC without the chip level synchronization. Furthermore, this work considers only small scale fading effects and does not consider large scale fading effects. In other words, perfect power control is assumed to compensate for the large scale effects, but no power control is assumed for the small scale effects. Investigating overall system performance when there is perfect power control for both large scale and small scale effects could be part of a continuation of this work. For simplicity, an uncoded DSCDMA system has been considered in this thesis. In practical systems, channel coding (for example forward error correction (FEC)), would be used to allow acceptable error rate performance at lower SINR ratio levels improving overall system performance. Studies on how error correction coding/decoding could be integrated into the DBSIC receiver structure could be another line of research, focusing on how DBSIC could take advantage of feedback soft information from the decoder.

Fig. 6.13 and Fig. 6.25 showed that DBSIC outperforms the other detectors including MF, SIC significantly in the case of detecting with random ordering (which means the detection does not necessarily start from the user with the strongest received power), showing that DBSIC has better near-far resistance than the MF and SIC detectors. One way to improve performance of the conventional SIC is to use it in combination with a non-uniform power control system [92, 93, 94]. Given that DBSIC also uses successive interference cancellation strategy, the techniques proposed in $[92,93,94]$ could be modified to further enhance the performance of DBSIC as well. 
Chapter 5 presents BER and AME performance analyses for the case of perfect channel estimation. Investigating the extension of the results to the case where channel estimation is not perfect is also of interest. Section 6.5 presents performance of the multiuser detectors assuming a non linear model for the channel estimation error. New studies considering a linear Gaussian model for the channel estimation error are a continuation of this work. A more exhaustive analysis of the performance of the proposed joint channel estimation and multiuser detector could also be done, looking for example at the effect of the number of iterations on the overall system performance. Other techniques can also be considered to be combined with DBSIC to improve the overall performance of CDMA systems. Receiver antenna diversity is a well-known effective technique to improve BER performance. The space-time coded transmit diversity offers a way to introduce a degree of space diversity without the complexity of closed-loop transmit diversity solutions. Sectored/adaptive antennas/smart antennas can enhance the desired signal and some fraction of the MAI and decrease the number of interferers. 


\section{Appendix A}

\section{Computational Complexity}

In general one real addition is counted as one flop operation [55], one real multiplication is counted as one [55] or a few flop operations. Division and square root are more complicated and usually counted as multiple of flops. Therefore, in this work we count the number of multiplications, additions/subtractions, divisions and square roots separately in calculating the computational complexity. In the evaluation of the receivers complexity, we count only the operations after the matched filters' outputs (i.e. starting from (3.15)) [132].

1. Decorrelating:

Starting from (3.15), the required operations are the inversion of $\overline{\mathbf{R}} \overline{\mathbf{E}}$ and the multiplication of $[\overline{\mathbf{R}} \overline{\mathbf{E}}]^{-1}$ and $\vec{y}$. The number of operations for each symbol/user is composed of three parts: the multiplication of $\overline{\mathbf{R}}$ and $\overline{\mathbf{E}}, \overline{\mathbf{R}} \overline{\mathbf{E}}$, the inversion of $\overline{\mathbf{R}} \overline{\mathbf{E}},(\overline{\mathbf{R}} \overline{\mathbf{E}})^{-1}$, multiplication of $(\overline{\mathbf{R}} \overline{\mathbf{E}})^{-1}$ to the matched filter outputs, $(\overline{\mathbf{R}} \overline{\mathbf{E}})^{-1} \cdot \vec{y}$

(a) $\overline{\mathbf{R}} \overline{\mathbf{E}}$ :

As the matrix $\overline{\mathbf{E}}$ is a diagonal matrix, the total number of operations for this step is $\left(N K_{v}\right)^{2}$ multiplications. 
(b) Inversion of $\overline{\mathbf{R}} \overline{\mathbf{E}}$ :

The inversion can be obtained through Gauss-Jordan elimination. During the forward elimination process, the number of operations at the $i^{\text {th }}$ step (where $i$ is from 1 to $N K_{v}$ ) is: $N K_{v}$ divisions $+\left(N K_{v}-i\right)\left(N K_{v}\right)$ multiplications $+\left(N K_{v}-i\right)\left(N K_{v}\right)$ additions. So the total number of operations for forward elimination process can be obtained as: $\left(N K_{v}\right)^{2}$ divisions + $\frac{1}{2}\left(N K_{v}-1\right)\left(N K_{v}\right)^{2}$ multiplications $+\frac{1}{2}\left(N K_{v}-1\right)\left(N K_{v}\right)^{2}$ additions.

The number of operations at the $i^{\text {th }}$ step for backward elimination process (where $i$ is from 1 to $\left.N K_{v}\right)$ is: $\left(N K_{v}-i\right)\left(N K_{v}\right)$ multiplications $+\left(N K_{v}-\right.$ $i)\left(N K_{v}\right)$ additions. So the total number of operations can be obtained as: $\frac{1}{2}\left(N K_{v}-1\right)\left(N K_{v}\right)^{2}$ multiplications $+\frac{1}{2}\left(N K_{v}-1\right)\left(N K_{v}\right)^{2}$ additions.

Combining the forward and backward process, the total number of operations for matrix inversion is: $\left(N K_{v}\right)^{2}$ divisions $+\left(N K_{v}-1\right)\left(N K_{v}\right)^{2}$ multiplications $+\left(N K_{v}-1\right)\left(N K_{v}\right)^{2}$ additions.

(c) $(\overline{\mathbf{R}} \overline{\mathbf{E}})^{-1} \cdot \vec{y}$ The number of operations for each row (with total $N K_{v}$ rows) is: $N K_{v}$ multiplications $+\left(N K_{v}-1\right)$ additions. So the total number of operations for this step is: $\left(N K_{v}\right)^{2}$ multiplications $+\left(N K_{v}-1\right) N K_{v}$ additions.

Therefore the computational complexity for the decorrelating detector can be obtained as (considering parts $(a),(b)$ and $(c)$ )

$\left(N K_{v}\right)^{2}$ (divisions) $+\left(N K_{v}+1\right)\left(N K_{v}\right)^{2}$ (multiplications) $+\left[\left(N K_{v}\right)^{2}-1\right]\left(N K_{v}\right)$ (additions). 
2. PIC with $g$ IC stages ( + one non IC stage):

There is no multiplication/addition/subtraction in the decision rule for the first stage (non IC stage). The number of operations to calculate $\hat{b}_{k, \mathrm{PIC}}^{(2)}=$ $\operatorname{sgn}\left(\Re\left\{y_{k}^{\mathrm{MF}}-\sum_{j=1, j \neq k}^{N K_{v}} z_{k j}^{\mathrm{MF}} \hat{b}_{j, \mathrm{PIC}}^{(1)}\right\}\right)$ for the first IC second stage is $\left(N K_{v}-1\right)$ multiplications $+\left(N K_{v}-1\right)$ additions/subtractions. So for all $k=1, \ldots, N K_{v}$, the total number of operations for 1 stage is: $\left(N K_{v}-1\right) N K_{v}$ multiplications + $\left(N K_{v}-1\right) N K_{v}$ additions/subtractions.

so the total complexity for $g$ IC stages is

$$
\left.g\left(N K_{v}-1\right) N K_{v} \text { (multiplications }\right)+g\left(N K_{v}-1\right) N K_{v}(\text { additions/subtractions })
$$

3. Reordering process for the conventional SIC, decorrelating DF and DBSIC:

For the conventional SIC, decorrelating DF and DBSIC, for each sequence of length $N$, the received signal is passed through the bank of matched filters once and the SIC cancellation/detecting order is determined based on these outputs for all the symbols of the sequence. Let $\vec{y}_{n e w}$ be the vector obtained by reordering $\vec{y}$ so that its elements $y_{i}$ are listed in decreasing order. Then

$$
\begin{aligned}
\vec{y}_{\text {new }} & =\overline{\mathbf{R}}_{\text {new }} \overline{\mathbf{E}}_{\text {new }} \vec{b}_{\text {new }}+\vec{n}_{\text {new }}=\overline{\mathbf{Z}} \vec{b}_{\text {new }}+\vec{n}_{\text {new }} \\
y_{i} & =\sum_{j=1}^{N K_{v}} z_{i j} b_{j}+n_{i} \quad i=1, \cdots, N K_{v}
\end{aligned}
$$

with $\overline{\mathbf{Z}}=\overline{\mathbf{R}}_{\text {new }} \overline{\mathbf{E}}_{\text {new }}$ and $b_{j}=j^{\text {th }}$ element of $\vec{b}_{\text {new }}$. So there are two similar operation processes for conventional SIC, DF and DBSIC, the creation of $\overline{\mathbf{Z}}$ and the ordering of $\vec{y}$.

(a) The total number of operations for the creation of $\overline{\mathbf{Z}}$ is: $\left(N K_{v}\right)^{2}$ multipli- 
cations.

(b) The total number of operations for the reordering of $\vec{y}$ is: $N K_{v} \log _{2}\left(N K_{v}\right)$ additions (we treat the complexity of reordering process as equal to the operation of addition).

4. Conventional SIC:

The number of operations for the computation of $\hat{b}_{k, \text { SIC }}=\operatorname{sgn}\left(\Re\left\{y_{k}-\sum_{j=1}^{k-1} z_{k j} \hat{b}_{j, \text { SIC }}\right\}\right)$ is $(k-1)$ multiplications $+(k-1)$ additions. Therefore the number of operations for all $k=1, \ldots, N K_{v}$ is

$$
\begin{aligned}
& \sum_{k=2}^{N K_{v}}(k-1) \text { (multiplications) }+\sum_{k=2}^{N K_{v}}(k-1) \text { (additions) } \\
& =\frac{1}{2}\left(N K_{v}-1\right) N K_{v} \text { (multiplications) }+\frac{1}{2}\left(N K_{v}-1\right) N K_{v} \text { (additions) }
\end{aligned}
$$

Combining with $3(\mathrm{a})$ and $3(\mathrm{~b})$, the total number of operations for conventional $\mathrm{SIC}$ is

$\frac{1}{2}\left(3 N K_{v}-1\right) N K_{v}($ multiplications $)+\left\{\frac{1}{2}\left(N K_{v}-1\right) N K_{v}+N K_{v} \log _{2}\left(N K_{v}\right)\right\}$ (additions)

5. Decorrelating DF:

Starting from (A.1), the number of operations for the decorrelating DF detector is composed of four parts: Cholesky decomposition of $\overline{\mathbf{Z}}\left(\overline{\mathbf{Z}}=\mathbf{F}^{\mathbf{T}} \mathbf{F}\right)$; inversion of $\mathbf{F}^{\mathbf{T}}$ (upper triangular matrix); multiplication of $\mathbf{F}^{T-1}$ to $\vec{y}_{\text {new }}$, and feedback filtering process, $\hat{b}_{k, \mathrm{DF}}=\operatorname{sgn}\left(\Re\left\{y_{f, k}-\sum_{j=1}^{k-1} F_{k j} \hat{b}_{j, \mathrm{DF}}\right\}\right)$.

(a) Cholesky decomposition of $\overline{\mathbf{Z}}$ : For $i=1, \ldots, N K_{v}, j=i+1, \ldots, N K_{v}$, $f_{i i}=\sqrt{\left(z_{i i}-\sum_{k=1}^{i-1} f_{i k}^{2}\right)}, f_{j i}=\left(z_{j i}-\sum_{k=1}^{i-1} f_{j k} f_{i k}\right) / f_{i i}$. 
The number of operations for the $i^{\text {th }}$ row can be obtained as: 1 square root $+\left(N K_{v}-i\right)$ divisions $+\left(N K_{v}-i+1\right)(i-1)$ multiplications $+\left(N K_{v}-\right.$ $i+1)(i-1)$ additions. So the total number of operations for Cholesky decomposition is: $\left(N K_{v}\right)$ square roots $+\frac{1}{2}\left(N K_{v}-1\right) N K_{v}$ divisions + $\frac{1}{6}\left[\left(N K_{v}\right)^{2}-1\right] N K_{v}$ multiplications $+\frac{1}{6}\left[\left(N K_{v}\right)^{2}-1\right] N K_{v}$ additions.

(b) Inversion of $\mathbf{F}^{\mathbf{T}}$ (upper triangular matrix): Taking into consideration that $\mathbf{F}^{T}$ is an upper triangular matrix, only the backward elimination process is needed for the inversion. The number of operations for the $i^{t h}$ step is: $i$ divisions $+\left(N K_{v}-i\right) i$ multiplications $+\left(N K_{v}-i\right)(i-1)$ additions. And the total number of operations for this matrix inversion can be obtained as: $\frac{1}{2}\left(N K_{v}+1\right) N K_{v}$ divisions $+\frac{1}{6}\left[\left(N K_{v}\right)^{2}-1\right] N K_{v}$ multiplications + $\frac{1}{6} N K_{v}\left(N K_{v}-1\right)\left(N K_{v}-2\right)$ additions.

(c) Multiplication of $\mathbf{F}^{T-1}$ to $\vec{y}_{\text {new }}$ (taking into consideration that $\mathbf{F}^{T-1}$ is an upper triangular matrix):

The number of operations for the multiplication of the $i^{\text {th }}$ row is: $\left(N K_{v}-\right.$ $i+1)$ multiplications $+\left(N K_{v}-i\right)$ additions. Therefore the total number of operations for the entire matrix multiplication is: $\frac{1}{2}\left(N K_{v}+1\right) N K_{v}$ multiplications $+\frac{1}{2} N K_{v}\left(N K_{v}-1\right)$ additions.

(d) The number of operation to calculate $\hat{b}_{k, \mathrm{DF}}=\operatorname{sgn}\left(\Re\left\{y_{f, k}-\sum_{j=1}^{k-1} F_{k j} \hat{b}_{j, \mathrm{DF}}\right\}\right)$. This process is similar to the conventional SIC, and the total number of operations can be obtained as: $\frac{1}{2}\left(N K_{v}-1\right) N K_{v}$ (multiplications) $+\frac{1}{2}\left(N K_{v}-\right.$ 1) $N K_{v}$ (additions)

Combining 5(a) to $5(\mathrm{~d})$ with $3(\mathrm{a})$ and $3(\mathrm{~b})$, the total number of operations for 
the decorrelating DF detector is

$$
\begin{gathered}
\left(N K_{v}\right)(\text { square roots })+\left(N K_{v}\right)^{2} \text { (divisions) } \\
+\frac{1}{3} N K_{v}\left[\left(N K_{v}\right)^{2}+6 N K_{v}-1\right](\text { multiplications }) \\
+\left\{\frac{1}{6} N K_{v}\left(N K_{v}-1\right)\left(2 N K_{v}+5\right)+N K_{v} \log _{2}\left(N K_{v}\right)\right\} \text { (additions) }
\end{gathered}
$$

\section{DBSIC:}

Starting from (A.1), the number of operations for DBSIC is composed of three parts: the matrix inversion of $\overline{\mathbf{Z}}^{(k)}$ where $k=1, \ldots, N K_{v}$; the multiplication of $\overline{\mathbf{Z}}^{(k)}$ to $\vec{y}_{\text {new }}^{(k)}\left(k=1, \ldots, N K_{v}\right)$, and the generation of $\vec{y}_{\text {new }}^{(k+1)},\left(k=1, \ldots, N K_{v}-\right.$ 1).

(a) Inversion of $\overline{\mathbf{Z}}^{(k)}$ where $k=1, \ldots, N K_{v}$ : According to the derivation for the decorrelating detector, the number of operations needed for the inversion of $\overline{\mathbf{Z}}^{(k)}$ is: $k^{2}$ divisions $+(k-1) k^{2}$ multiplications $+(k-1) k^{2}$ additions. Therefore the total number of operations for the matrix inversions in DBSIC can be obtained as: $\frac{1}{6} N K_{v}\left(N K_{v}+1\right)\left(2 N K_{v}+1\right)$ (divisions) $+\left\{\frac{1}{4}\left(N K_{v}\right)^{2}\left(N K_{v}+1\right)^{2}-\frac{1}{6} N K_{v}\left(N K_{v}+1\right)\left(2 N K_{v}+1\right)\right\}$ (multiplications)+ $\left\{\frac{1}{4}\left(N K_{v}\right)^{2}\left(N K_{v}+1\right)^{2}-\frac{1}{6} N K_{v}\left(N K_{v}+1\right)\left(2 N K_{v}+1\right)\right\}$ (additions).

(b) Multiplication of $\overline{\mathbf{Z}}^{(k)}$ to $\vec{y}_{\text {new }}^{(k)}\left(k=1, \ldots, N K_{v}\right)$ : The number of operations for the multiplication of $\overline{\mathbf{Z}}^{(k)}$ to $\vec{y}_{\text {new }}^{(k)}$ is: $k$ multiplications $+(k-1)$ additions. Therefore the total number of operations for this part can be obtained as: $\frac{1}{2}\left(N K_{v}+1\right) N K_{v}$ (multiplications $)+\frac{1}{2}\left(N K_{v}-1\right) N K_{v}$ (additions).

(c) Generation of $\vec{y}_{\text {new }}^{(k+1)},\left(k=1, \ldots, N K_{v}-1\right)$ : The number of operations for the generation of $\vec{y}_{\text {new }}^{(k+1)}$ is: $N K_{v}-k$ multiplications $+N K_{v}-k$ additions. 
Therefore the total number of operations for this part can be obtained as: $\frac{1}{2}\left(N K_{v}-1\right) N K_{v}$ (multiplications) $+\frac{1}{2}\left(N K_{v}-1\right) N K_{v}$ (additions).

Combining $6(\mathrm{a})$ to $6(\mathrm{c})$ with $3(a)$ and $3(b)$, the total number of operations for DBSIC is

$$
\begin{gathered}
\frac{1}{6} N K_{v}\left(N K_{v}+1\right)\left(2 N K_{v}+1\right) \text { (divisions) } \\
\left.+\frac{1}{12} N K_{v}\left[3\left(N K_{v}\right)^{3}+2\left(N K_{v}\right)^{2}+21 N K_{v}-2\right)\right] \text { (multiplications) } \\
+\left\{\frac{1}{12} N K_{v}\left[3\left(N K_{v}\right)^{3}+2\left(N K_{v}\right)^{2}+9 N K_{v}-2\right]+N K_{v} \log _{2}\left(N K_{v}\right)\right\} \text { (additions) }
\end{gathered}
$$

7. Linear decorrelating detector with reduced complexity:

Starting from (3.32), the number of operations for the linear decorrelating detector with reduced complexity is composed of three parts: the matrix inversion of $\overline{\mathbf{Z}}^{(k)}$ where $k=1, \ldots, N K_{v}$; the multiplication of $\overline{\mathbf{Z}}^{(k)}$ to $\bar{y}_{\text {new }}^{(k)}\left(k=1, \ldots, N K_{v}\right)$, and the generation of $\vec{y}_{\text {new }}^{(k+1)},\left(k=1, \ldots, N K_{v}-1\right)$.

(a) Cholesky decomposition of $\mathbf{Z}[\mathbf{0}]$ : The total number of operations for Cholesky decomposition is: $\left(K_{v}\right)$ square roots $+\frac{1}{2}\left(K_{v}-1\right) K_{v}$ divisions $+\frac{1}{6}\left[\left(K_{v}\right)^{2}-\right.$ 1] $K_{v}$ multiplications $+\frac{1}{6}\left[\left(K_{v}\right)^{2}-1\right] K_{v}$ additions.

(b) Inversion of $\mathbf{F}^{\mathrm{T}}$ [0] (upper triangular matrix): The total number of operations for this matrix inversion can be obtained as: $\frac{1}{2}\left(K_{v}+1\right) K_{v}$ divisions $+\frac{1}{6}\left[\left(K_{v}\right)^{2}-1\right] K_{v}$ multiplications $+\frac{1}{6} K_{v}\left(K_{v}-1\right)\left(K_{v}-2\right)$ additions.

(c) Multiplication of $\left(\mathbf{F}^{T}[0]\right)^{-1}$ to $\mathbf{R}[1]$ (and same step to find $\mathbf{F}[1]$ ): The total number of operations for the entire matrix multiplication is: $\frac{1}{6}\left[\left(K_{v}\right)^{2}-1\right] K_{v}$ multiplications $+\frac{1}{6} K_{v}\left(K_{v}-1\right)\left(K_{v}-2\right)$ additions. 
(d) Multiplication of $\mathbf{R}^{T}[1]\left\{\mathbf{F}^{T}[0]^{-1}\right\}^{T}\left\{\mathbf{F}^{T}[0]\right\}^{-1} \mathbf{R}[1]$ : The total number of operations for the entire matrix multiplication is: $\frac{1}{6} K_{v}\left(K_{v}-1\right)\left(2 K_{v}-1\right)$ multiplications $+\frac{1}{6}\left(K_{v}-1\right)\left(K_{v}-2\right)\left(2 K_{v}-3\right)$ additions.

(e) $\mathbf{F} \overline{\mathbf{E}}$ : The total number of operations for this step is $N\left(K_{v}\right)^{2}-\frac{1}{2}\left(K_{v}-1\right) K_{v}$ multiplications.

(f) Inversion of $\mathbf{F} \overline{\mathbf{E}}$ : The total number of operations for matrix inversion is: $\frac{1}{2}\left(N K_{v}+1\right) N K_{v}$ divisions $+\frac{1}{6}\left[\left(N K_{v}\right)^{2}-1\right] N K_{v}$ multiplications + $\frac{1}{6} N K_{v}\left(N K_{v}-1\right)\left(N K_{v}-2\right)$ additions.

(g) $(\mathbf{F} \overline{\mathbf{E}})^{-1} \cdot \vec{y}_{w}$ : The total number of operations for this step is: $\frac{1}{2}\left(N K_{v}+\right.$ 1) $N K_{v}$ multiplications $+\frac{1}{2} N K_{v}\left(N K_{v}-1\right)$ additions.

Considering three iterations in 7 (a) to $7(\mathrm{~d})$, the total number of operations for the linear decorrelating detector with reduced complexity is

$$
\begin{gathered}
3\left(K_{v}\right) \text { (square roots) }+\left\{\frac{1}{2}\left(N K_{v}+1\right) N K_{v}+3\left(K_{v}\right)^{2}\right\} \text { (divisions) } \\
+\left\{\frac{1}{6} N K_{v}\left[N K_{v}+1\right)\left(N K_{v}+2\right)+\frac{1}{2} K_{v}\left[5 K_{v}+1\right)\left(K_{v}-1\right)\right\} \text { (multiplications) } \\
+\left\{\frac{1}{6}\left[\left(K_{v}\right)^{2}-1\right] K_{v}+\frac{1}{2}\left(K_{v}-1\right)\left[5\left(K_{v}\right)^{2}-10 K_{v}+6\right]\right\} \text { (additions) }
\end{gathered}
$$




\section{Appendix B}

\section{DBSIC BER Performance Analysis}

Recall that

$$
\begin{aligned}
\vec{y}_{\text {new }}^{(k)} & =\overline{\mathbf{Z}}^{(\mathbf{k})} \vec{b}_{\text {new }}^{(k)}+\vec{n}_{\text {new }}^{(k)} \\
\hat{b}_{k, \text { DBSIC }} & =\operatorname{sgn}\left(\Re\left\{\left[\left\{\overline{\mathbf{Z}}^{(\mathbf{k})}\right\}^{-1} \vec{y}_{\text {new }}^{(k)}\right]_{1}\right\}\right)
\end{aligned}
$$

Let $\mathbf{V}^{(k)}=\left\{\overline{\mathbf{Z}}^{(\mathbf{k})}\right\}^{-1}$, substituting (B.1) into (B.2) yields

$$
\begin{aligned}
\hat{b}_{k, \mathrm{DBSIC}} & =\operatorname{sgn}\left(\Re\left\{\left[\vec{b}_{\text {new }}^{(k)}+\mathbf{V}^{(k)} \vec{n}_{\text {new }}^{(k)}\right]_{1}\right\}\right)=\operatorname{sgn}\left(\Re\left\{b_{k}+\sum_{i=1}^{N K_{v}-k+1} v_{1 i}^{(k)} n_{k+i-1}^{(k)}\right\}\right) \\
& =\operatorname{sgn}\left(b_{k}+\Re\left\{\sum_{i=1}^{N K_{v}-k+1} v_{1 i}^{(k)} n_{i+k-1}\right\}+\sum_{i=1}^{N K_{v}-k+1} \sum_{j=1}^{k-1} \Re\left\{v_{1 i}^{(k)} z_{i+k-1 j}\right\}\left(b_{j}-\hat{b}_{j}\right)\right)
\end{aligned}
$$

Following [43] we assume that $\Re\left\{\sum_{i=1}^{N K_{v}-k+1} v_{1 i}^{(k)} n_{i+k-1}\right\}$ and $\left(b_{j}-\hat{b}_{j}\right)$ are independent Gaussian random variables. Since all terms of the summation are independent, 
$\Re\left\{\sum_{i=1}^{N K_{v}-k+1} v_{1 i}^{(k)} n_{k+i-1}^{(k)}\right\}$ has zero mean, and its variance is given by:

$$
\begin{aligned}
& \operatorname{Var}\left[\Re\left\{\sum_{i=1}^{N K_{v}-k+1} v_{1 i}^{(k)} n_{k+i-1}^{(k)}\right\} \mid \vec{\alpha}\right] \\
& =\operatorname{Var}\left[\Re\left\{\sum_{i=1}^{N K_{v}-k+1} v_{1 i}^{(k)} n_{i+k-1}\right\} \mid \vec{\alpha}\right]+\operatorname{Var}\left[\sum_{i=1}^{N K_{v}-k+1} \sum_{j=1}^{k-1} \Re\left\{v_{1 i}^{(k)} z_{i+k-1}\right\}\left(\hat{b}_{j}-b_{j}\right) \mid \vec{\alpha}\right] \\
& z_{k l}=[\overline{\mathbf{Z}}]_{k l}=\left[\overline{\mathbf{R}}_{\text {new }} \overline{\mathbf{E}}_{\text {new }}\right]_{k l}=\sum_{j=1}^{N K_{v}}\left[\overline{\mathbf{R}}_{\text {new }}\right]_{k j}\left[\overline{\mathbf{E}}_{\text {new }}\right]_{j l}=\left[\overline{\mathbf{R}}_{\text {new }}\right]_{k l}\left[\overline{\mathbf{E}}_{\text {new }}\right]_{l l}=\left[\overline{\mathbf{R}}_{\text {new }}\right]_{k l} \epsilon_{l}
\end{aligned}
$$

yielding

$$
E\left[n_{k} n_{l}^{*} / 2 \mid \vec{\alpha}\right]=\frac{N_{0}}{2}\left[\overline{\mathbf{R}}_{\text {new }}\right]_{k l}=\frac{N_{0}}{2 \epsilon_{l}} z_{k l}=\frac{N_{0}}{2 \epsilon_{l}} z_{l k}^{*}
$$

where $\epsilon_{l}$ denotes the $l^{\text {th }}$ element of the diagonal matrix $\overline{\mathbf{E}}_{\text {new }}$. The conditional variance of $\Re\left\{\sum_{i=1}^{N K_{v}-k+1} v_{1 i}^{(k)} n_{i+k-1}\right\}$ can be calculated as follows:

$$
\begin{aligned}
& \operatorname{Var}\left[\Re\left\{\sum_{i=1}^{N K_{v}-k+1} v_{1 i}^{(k)} n_{i+k-1}\right\} \mid \vec{\alpha}\right] \\
& =\operatorname{Var}\left[\frac{1}{2}\left\{\sum_{i=1}^{N K_{v}-k+1} v_{1 i}^{(k)} n_{i+k-1}+\left(\sum_{i=1}^{N K_{v}-k+1} v_{1 i}^{(k)} n_{i+k-1}\right)^{*}\right\} \mid \vec{\alpha}\right] \\
& =\frac{1}{4} E\left[\left\{\sum_{i=1}^{N K_{v}-k+1} v_{1 i}^{(k)} n_{i+k-1}+\left(\sum_{i=1}^{N K_{v}-k+1} v_{1 i}^{(k)} n_{i+k-1}\right)^{*}\right\}^{2} \mid \vec{\alpha}\right] \\
& =\frac{1}{4} E\left[\left(\sum_{i=1}^{N K_{v}-k+1} v_{1 i}^{(k)} n_{i+k-1}\right)^{2} \mid \vec{\alpha}\right]+\frac{1}{4} E\left[\left(\left(\sum_{i=1}^{N K_{v}-k+1} v_{1 i}^{(k)} n_{i+k-1}\right)^{*}\right)^{2} \mid \vec{\alpha}\right] \\
& +\frac{1}{2} E\left[\left(\sum_{i=1}^{N K_{v}-k+1} v_{1 i}^{(k)} n_{i+k-1}\right)\left(\sum_{i=1}^{N K_{v}-k+1} v_{1 i}^{(k)} n_{i+k-1}\right)^{*} \mid \vec{\alpha}\right]
\end{aligned}
$$


Since $n_{i+k-1}$ is circularly complex, the first two terms are equal to zero, so it is obtained

$$
\begin{aligned}
& \operatorname{Var}\left[\Re\left\{\sum_{i=1}^{N K_{v}-k+1} v_{1 i}^{(k)} n_{i+k-1}\right\} \mid \vec{\alpha}\right] \\
& =\frac{1}{2} E\left[\left(\sum_{j=1}^{N K_{v}-k+1} v_{1 j}^{(k)} n_{j+k-1}\right)\left(\sum_{i=1}^{N K_{v}-k+1} v_{1 i}^{(k)} n_{i+k-1}\right)^{*} \mid \vec{\alpha}\right] \\
& =\sum_{j=1}^{N K_{v}-k+1} \sum_{i=1}^{N K_{v}-k+1} v_{1 j}^{(k)}\left(v_{1 i}^{(k)}\right)^{*} E\left[n_{j+k-1} n_{i+k-1}^{*} / 2 \mid \vec{\alpha}\right] \\
& =\sum_{j=1}^{N K_{v}-k+1} \sum_{i=1}^{N K_{v}-k+1} v_{1 j}^{(k)}\left(v_{1 i}^{(k)}\right)^{*} \frac{N_{0}}{2} \frac{1}{\epsilon_{i+k-1}} z_{j+k-1 i+k-1} \\
& =\frac{N_{0}}{2} \sum_{i=1}^{N K_{v}-k+1} \frac{1}{\epsilon_{i+k-1}}\left(v_{1 i}^{(k)}\right)^{*} \sum_{j=1}^{N K_{v}-k+1} v_{1 j}^{(k)} z_{j+k-1 i+k-1} \\
& =\frac{N_{0}}{2} \sum_{i=1}^{N K_{v}-k+1} \frac{1}{\epsilon_{i+k-1}}\left(v_{1 i}^{(k)}\right)^{*} \sum_{j=1}^{N K_{v}-k+1}\left[\boldsymbol{V}^{(k)}\right]_{1 j}\left[\overline{\mathbf{Z}}^{(\mathbf{k})}\right]_{j i} \\
& =\frac{N_{0}}{2} \sum_{i=1}^{N K_{v}-k+1} \frac{1}{\epsilon_{i+k-1}}\left(v_{1 i}^{(k)}\right)^{*}\left[\boldsymbol{V}^{(k)} \overline{\mathbf{Z}}^{(\mathbf{k})}\right]_{1 i} \\
& =\frac{N_{0}}{2} \sum_{i=1}^{N K_{v}-k+1} \frac{1}{\epsilon_{i+k-1}}\left(v_{1 i}^{(k)}\right)^{*}[\boldsymbol{I}]_{1 i} \\
& =\frac{N_{0}}{2} \frac{1}{\epsilon_{k}}\left(v_{11}^{(k)}\right)^{*}=\frac{N_{0}}{2} \frac{1}{\epsilon_{k}} v_{11}^{(k)}
\end{aligned}
$$

since similar to $\overline{\mathbf{Z}}^{(\mathbf{k})}, \boldsymbol{V}^{(k)}=\overline{\mathbf{Z}}^{(\mathbf{k})-\mathbf{1}}$ is Hermitian. 
Assuming zero mean uncorrelated decision errors, it is obtained

$$
\begin{aligned}
\operatorname{Var}\left[\Re\left\{\sum_{i=1}^{N K_{v}-k+1} \sum_{j=1}^{k-1} v_{1 i}^{(k)} z_{i+k-1 j}\left(\hat{b}_{j}-b_{j}\right)\right\} \mid \vec{\alpha}\right] \\
=\operatorname{Var}\left[\sum_{i=1}^{N K_{v}-k+1} \sum_{j=1}^{k-1} \Re\left\{v_{1 i}^{(k)} z_{i+k-1 j}\right\}\left(\hat{b}_{j}-b_{j}\right) \mid \vec{\alpha}\right] \\
=E\left[\left(\sum_{i=1}^{N K_{v}-k+1} \sum_{j=1}^{k-1} \Re\left\{v_{1 i}^{(k)} z_{i+k-1 j}\right\}\left(\hat{b}_{j}-b_{j}\right)\right)^{2} \mid \vec{\alpha}\right] \\
=E\left[\sum_{i=1}^{N K_{v}-k+1} \sum_{j=1}^{k-1} \Re\left\{v_{1 i}^{(k)} z_{i+k-1 j}\right\}\left(\hat{b}_{j}-b_{j}\right) \sum_{u=1}^{N K_{v}-k+1} \sum_{l=1}^{k-1} \Re\left\{v_{1 u}^{(k)} z_{u+k-1 l}\right\}\left(\hat{b}_{l}-b_{l}\right) \mid \vec{\alpha}\right] \\
=\sum_{i=1}^{N K_{v}-k+1} \sum_{j=1}^{k-1} \Re\left\{v_{1 i}^{(k)} z_{i+k-1 j}\right\} \sum_{u=1}^{N K_{v}-k+1} \sum_{l=1}^{k-1} \Re\left\{v_{1 u}^{(k)} z_{u+k-1 l}\right\} E\left[\left(\hat{b}_{j}-b_{j}\right)\left(\hat{b}_{l}-b_{l}\right) \mid \vec{\alpha}\right] \\
=\sum_{i=1}^{N K_{v}-k+1} \sum_{j=1}^{k-1} \sum_{u=1}^{N K_{v}-k+1} \Re\left\{v_{1 i}^{(k)} z_{i+k-1 j}\right\} \Re\left\{v_{1 u}^{(k)} z_{u+k-1 j}\right\} E\left[\left(\hat{b}_{j}-b_{j}\right)^{2} \mid \vec{\alpha}\right] \\
=4 \sum_{j=1}^{k-1} \sum_{i=1}^{N K_{v}-k+1} \Re\left\{v_{1 i}^{(k)} z_{i+k-1}\right\} \sum_{u=1}^{N K_{v}-k+1} \Re\left\{v_{1 u}^{(k)} z_{u+k-1 j}\right\} P_{e, j \mid \vec{\alpha}} \\
\left.=4 \sum_{j=1}^{k-1}\left(\sum_{i=1}^{N K_{v}-k+1} \Re\left\{v_{1 i}^{(k)} z_{i+k-1 j}\right\}\right)^{2} P_{e, j \mid \vec{\alpha}}=4 \sum_{j=1}^{k-1} \Re\left\{\sum_{i=1}^{N K_{v}-k+1} v_{1 i}^{(k)} z_{i+k-1 j}\right\}\right)^{2} P_{e, j \mid \vec{\alpha}}
\end{aligned}
$$

since it can also be shown that $E\left\{\left(b_{j}-\hat{b}_{j}\right)^{2} \mid \vec{\alpha}\right\}=0 \cdot\left(1-P_{e, j \mid \vec{\alpha}}\right)+\left(2 b_{j}\right)^{2} \cdot P_{e, j \mid \vec{\alpha}}=4 P_{e, j \mid \vec{\alpha}}$ from $\operatorname{Pr}\left\{\left(b_{j}-\hat{b}_{j}\right)=0 \mid b_{j}, \vec{\alpha}\right\}=1-P_{e, j \mid \vec{\alpha}}$ and $\operatorname{Pr}\left\{\left(b_{j}-\hat{b}_{j}\right)=2 b_{j} \mid b_{j}, \vec{\alpha}\right\}=P_{e, j \mid \vec{\alpha}}$.

$$
P_{e, k \mid \vec{\alpha}, \mathrm{DBSIC}} \approx Q\left(\left[v_{11}^{(k)} \frac{N_{0}}{2 \epsilon_{k}}+4 \sum_{j=1}^{k-1}\left(\Re\left\{\sum_{i=1}^{N K_{v}-k+1} v_{1 i}^{(k)} z_{i+k-1 j}\right\}\right)^{2} P_{e, j \mid \vec{\alpha}}\right]^{-\frac{1}{2}}\right)
$$

with $P_{e, 1 \mid \vec{\alpha}, \mathrm{DBSIC}}=Q\left(\left[v_{11}^{(1)} \frac{N_{0}}{2 \epsilon_{1}}\right]^{-\frac{1}{2}}\right)$ 


\section{Appendix C}

\section{Conventional SIC BER}

\section{Performance Analysis}

For the conventional SIC, the symbol estimate can be written as $\hat{b}_{k, \text { SIC }}=\operatorname{sgn}\left(\Re\left\{y_{k}-\sum_{j=1}^{k-1} z_{k j} \hat{b}_{j, \text { SIC }}\right\}\right)$, or equivalently as

$$
\begin{aligned}
\hat{b}_{k, \mathrm{SIC}}^{(k)} & =\operatorname{sgn}\left(\Re\left\{z_{k k}^{-1}\left[y_{k}-\sum_{j=1}^{k-1} z_{k j} \hat{b}_{j}\right]\right\}\right) \\
& =\operatorname{sgn}\left(b_{k}+\sum_{j=k+1}^{N K_{v}} \Re\left\{z_{k j} z_{k k}^{-1}\right\} b_{j}+\Re\left\{z_{k k}^{-1} n_{k}\right\}+\sum_{j=1}^{k-1} \Re\left\{z_{k j} z_{k k}^{-1}\right\}\left(b_{j}-\hat{b}_{j}\right)\right)
\end{aligned}
$$

$$
\begin{aligned}
& \operatorname{Var}\left[\Re\left\{\sum_{j=k+1}^{N K_{v}} z_{k j} z_{k k}^{-1} b_{j}+z_{k k}^{-1} n_{k}+\sum_{j=1}^{k-1} z_{k j} z_{k k}^{-1}\left(b_{j}-\hat{b}_{j}\right)\right\} \mid \vec{\alpha}\right] \\
& =\operatorname{Var}\left[\sum_{j=k+1}^{N K_{v}} \Re\left\{z_{k j} z_{k k}^{-1}\right\} b_{j}+\Re\left\{z_{k k}^{-1} n_{k}\right\}+\sum_{j=1}^{k-1} \Re\left\{z_{k j} z_{k k}^{-1}\right\}\left(b_{j}-\hat{b}_{j}\right) \mid \vec{\alpha}\right] \\
& =\operatorname{Var}\left[\sum_{j=k+1}^{N K_{v}} \Re\left\{z_{k j} z_{k k}^{-1}\right\} b_{j}+\sum_{j=1}^{k-1} \Re\left\{z_{k j} z_{k k}^{-1}\right\}\left(b_{j}-\hat{b}_{j}\right) \mid \vec{\alpha}\right]+\operatorname{Var}\left[\Re\left\{z_{k k}^{-1} n_{k}\right\} \mid \vec{\alpha}\right]
\end{aligned}
$$




$$
\begin{aligned}
\operatorname{Var}\left[\Re\left\{z_{k k}^{-1} n_{k}\right\} \mid \vec{\alpha}\right] & =\operatorname{Var}\left[\frac{1}{2}\left[z_{k k}^{-1} n_{k}+z_{k k}^{-1 *} n_{k}^{*}\right] \mid \vec{\alpha}\right]=\frac{1}{4} E\left[\left(z_{k k}^{-1} n_{k}+z_{k k}^{-1 *} n_{k}^{*}\right)^{2} \mid \vec{\alpha}\right] \\
& =\frac{1}{4} E\left[\left\{z_{k k}^{-1} n_{k}\right\}^{2} \mid \vec{\alpha}\right]+\frac{1}{4} E\left[\left\{z_{k k}^{-1 *} n_{k}^{*}\right\}^{2} \mid \vec{\alpha}\right]+\frac{1}{2} E\left[\left|z_{k k}^{-1} n_{k}\right|^{2}\right]
\end{aligned}
$$

Since $n_{i+k-1}$ is circularly complex, the first two terms are equal to zero, so we obtain

$$
\begin{aligned}
& \operatorname{Var}\left[\Re\left\{z_{k k}^{-1} n_{k}\right\} \mid \vec{\alpha}\right]=\frac{1}{2} E\left[\left|z_{k k}^{-1} n_{k}\right|^{2} \mid \vec{\alpha}\right]=\left|z_{k k}^{-1}\right|^{2} E\left[\left|n_{k}\right|^{2} / 2 \mid \vec{\alpha}\right] \\
& =\left|z_{k k}^{-1}\right|^{2} \frac{N_{0}}{2} \frac{z_{k k}}{\epsilon_{k}}=z_{k k}^{-1 *} \frac{N_{0}}{2 \epsilon_{k}}=z_{k k}^{-1} \frac{N_{0}}{2 \epsilon_{k}} \\
& \operatorname{Var}\left[\sum_{j=k+1}^{N K_{v}} \Re\left\{z_{k j} z_{k k}^{-1}\right\} b_{j}+\sum_{j=1}^{k-1} \Re\left\{z_{k j} z_{k k}^{-1}\right\}\left(b_{j}-\hat{b}_{j}\right) \mid \vec{\alpha}\right] \\
& =E\left[\left(\sum_{j=k+1}^{N K_{v}} \Re\left\{z_{k j} z_{k k}^{-1}\right\} b_{j}+\sum_{j=1}^{k-1} \Re\left\{z_{k j} z_{k k}^{-1}\right\}\left(b_{j}-\hat{b}_{j}\right)\right)^{2} \mid \vec{\alpha}\right] \\
& =E\left[\left(\sum_{j=k+1}^{N K_{v}} \Re\left\{z_{k j} z_{k k}^{-1}\right\} b_{j}\right)^{2} \mid \vec{\alpha}\right]+E\left[\left(\sum_{j=1}^{k-1} \Re\left\{z_{k j} z_{k k}^{-1}\right\}\left(b_{j}-\hat{b}_{j}\right)\right)^{2} \mid \vec{\alpha}\right] \\
& +2 E\left[\sum_{j=k+1}^{N K_{v}} \Re\left\{z_{k j} z_{k k}^{-1}\right\} b_{j} \sum_{l=1}^{k-1} \Re\left\{z_{k l} z_{k k}^{-1}\right\}\left(b_{l}-\hat{b}_{l}\right)\right] \\
& =\sum_{j=k+1}^{N K_{v}} \Re\left\{z_{k j} z_{k k}^{-1}\right\} \sum_{l=k+1}^{N K_{v}} \Re\left\{z_{k l} z_{k k}^{-1}\right\} E\left[b_{j} b_{l} \mid \vec{\alpha}\right] \\
& +\sum_{j=1}^{k-1} \Re\left\{z_{k j} z_{k k}^{-1}\right\} \sum_{l=1}^{k-1} \Re\left\{z_{k l} z_{k k}^{-1}\right\} E\left[\left(b_{j}-\hat{b}_{j}\right)\left(b_{l}-\hat{b}_{l}\right) \mid \vec{\alpha}\right] \\
& +2 \sum_{j=k+1}^{N K_{v}} \Re\left\{z_{k j} z_{k k}^{-1}\right\} \sum_{l=1}^{k-1} \Re\left\{z_{k l} z_{k k}^{-1}\right\} E\left[b_{j}\left(b_{l}-\hat{b}_{l}\right) \mid \vec{\alpha}\right]
\end{aligned}
$$

Assuming uncorrelated data and data uncorrelated with the decision error, we 
obtain

$$
\begin{aligned}
& \operatorname{Var}\left[\sum_{j=k+1}^{N K_{v}} \Re\left\{z_{k j} z_{k k}^{-1}\right\} b_{j}+\sum_{j=1}^{k-1} \Re\left\{z_{k j} z_{k k}^{-1}\right\}\left(b_{j}-\hat{b}_{j}\right) \mid \vec{\alpha}\right] \\
& =\sum_{j=k+1}^{N K_{v}}\left(\Re\left\{z_{k j} z_{k k}^{-1}\right\}\right)^{2}+\sum_{j=1}^{k-1}\left(\Re\left\{z_{k j} z_{k k}^{-1}\right\}\right)^{2} E\left[\left(b_{j}-\hat{b}_{j}\right)^{2} \mid \vec{\alpha}\right] \\
& \left.=\sum_{j=k+1}^{N K_{v}}\left(\Re\left\{z_{k j} z_{k k}^{-1}\right\}\right)^{2}+4 \sum_{j=1}^{k-1}\left(\Re\left\{z_{k j} z_{k k}^{-1}\right\}\right)^{2} P_{e, j \mid \vec{\alpha}} \quad \text { (since } E\left\{\left(b_{j}-\hat{b}_{j}\right)^{2} \mid \vec{\alpha}\right\}=4 P_{e, j \mid \vec{\alpha}}\right)
\end{aligned}
$$

$$
\begin{aligned}
& \operatorname{Var}\left[\Re\left\{z_{k k}^{-1} n_{k}\right\}+\sum_{j=k+1}^{N K_{v}} \Re\left\{z_{k j} z_{k k}^{-1}\right\} b_{j}+\sum_{j=1}^{k-1} \Re\left\{z_{k j} z_{k k}^{-1}\right\}\left(b_{j}-\hat{b}_{j}\right) \mid \vec{\alpha}\right] \\
& =z_{k k}^{-1} \frac{N_{0}}{2 \epsilon_{k}}+\sum_{j=k+1}^{N K_{v}}\left(\Re\left\{z_{k j} z_{k k}^{-1}\right\}\right)^{2}+4 \sum_{j=1}^{k-1}\left(\Re\left\{z_{k j} z_{k k}^{-1}\right\}\right)^{2} P_{e, j \mid \vec{\alpha}}
\end{aligned}
$$

Hence it is obtained that

$$
\begin{aligned}
P_{e, k \mid \vec{\alpha}, \mathrm{SIC}} & \approx Q\left(\left[z_{k k}^{-1} \frac{N_{0}}{2 \epsilon_{k}}+\sum_{j=k+1}^{N K_{v}}\left(\Re\left\{z_{k j} z_{k k}^{-1}\right\}\right)^{2}+4 \sum_{j=1}^{k-1}\left(\Re\left\{z_{k j} z_{k k}^{-1}\right\}\right)^{2} P_{e, j \mid \vec{\alpha}}\right]^{-\frac{1}{2}}\right) \\
\text { with } \quad P_{e, 1 \mid \vec{\alpha}, \mathrm{SIC}} & =Q\left(\left[z_{11}^{-1} \frac{N_{0}}{2 \epsilon_{1}}+\sum_{j=2}^{N K_{v}}\left(\Re\left\{z_{1 j} z_{11}^{-1}\right\}\right)^{2}\right]^{-\frac{1}{2}}\right)
\end{aligned}
$$




\section{Appendix D}

\section{AME Performance Analysis of DBSIC for AWGN Channels}

For the AWGN channel and considering 2 users, the matched filter outputs (5.3) are simplified as:

$$
\begin{gathered}
\vec{y}^{\mathrm{s}}=\left(\begin{array}{l}
y_{1}^{\mathrm{s}} \\
y_{2}^{\mathrm{s}}
\end{array}\right)=\boldsymbol{R}_{\mathrm{s}} \boldsymbol{E}_{\mathrm{s}} \vec{b}^{\mathrm{s}}+\vec{n}^{\mathrm{s}}=\left(\begin{array}{cc}
1 & \rho \\
\rho & 1
\end{array}\right)\left(\begin{array}{cc}
\sqrt{E_{1}^{s}} & 0 \\
0 & \sqrt{E_{2}^{s}}
\end{array}\right)\left(\begin{array}{l}
b_{1}^{\mathrm{s}} \\
b_{2}^{\mathrm{s}}
\end{array}\right)+\left(\begin{array}{c}
n_{1}^{\mathrm{s}} \\
n_{2}^{\mathrm{s}}
\end{array}\right) \\
\left\{\begin{array}{l}
y_{1}^{\mathrm{s}}=\sqrt{E_{1}^{\mathrm{s}}} b_{1}^{\mathrm{s}}+\sqrt{E_{2}^{\mathrm{s}}} \rho b_{2}^{\mathrm{s}}+n_{1}^{\mathrm{s}} \\
y_{2}^{\mathrm{s}}=\sqrt{E_{1}^{s}} \rho b_{1}^{\mathrm{s}}+\sqrt{E_{2}^{s}} b_{2}^{\mathrm{s}}+n_{2}^{\mathrm{s}}
\end{array}\right.
\end{gathered}
$$

where

$$
\rho=\int_{0}^{T_{s}} S_{1}(t) S_{2}^{*}(t) d t
$$

is the cross-correlation between user 1 and user 2 (assumed to be real for simplicity), and $\vec{n}^{\mathrm{s}}=\left(n_{1}^{\mathrm{s}}, n_{2}^{\mathrm{s}}\right)^{T}$ has zero mean and autocorrelation function $E\left[\vec{n}^{\mathrm{s}} \vec{n}^{\mathrm{s}^{\dagger}} / 2\right]=$ $\left(N_{0} / 2\right) \boldsymbol{R}_{\mathrm{s}}$ 
For simplicity we assume that user 1 is the first user to be canceled (no reordering done, $\vec{y}_{\text {new }}^{\mathrm{s}}=\vec{y}^{\mathrm{s}}, \vec{b}_{\text {new }}^{\mathrm{s}}=\vec{b}^{\mathrm{s}}$ ). Since the estimate of the first bit using DBSIC is the same as the one that would be obtained with the decorrelating detector, we have

$$
\eta_{1}=\eta_{1, \mathrm{dec}}=1-\rho^{2}
$$

To obtain $\eta_{2}$, we can start to compute the BER $P_{e, 2}\left(N_{0}\right)$ for the second user. For DBSIC,

$$
\begin{aligned}
y_{2}^{\mathrm{s}(2)} & =y_{2}^{\mathrm{s}(1)}-\sqrt{E_{1}^{s}} \rho \hat{b}_{1}^{\mathrm{s}}=y_{2}^{\mathrm{s}}-\sqrt{E_{1}^{s}} \rho \hat{b}_{1}^{\mathrm{s}} \\
& =\sqrt{E_{2}^{s}} b_{2}^{\mathrm{s}}+n_{2}^{\mathrm{s}}+\sqrt{E_{1}^{s}} \rho\left(b_{1}^{\mathrm{s}}-\hat{b}_{1}^{\mathrm{s}}\right) \quad \text { using (D.1) } \\
\hat{b}_{2}^{\mathrm{s}} & =\operatorname{sgn}\left(\Re\left\{\left[\left(\mathbf{Z}_{\mathrm{s}}^{(2)}\right)^{-1} \vec{y}^{\mathrm{s}(2)}\right]_{1}\right\}\right) \\
& =\operatorname{sgn}\left(\Re \left\{b_{2}^{\mathrm{s}}+\frac{1}{\sqrt{E_{2}^{s}}} n_{2}^{\mathrm{s}}+\sqrt{\left.\left.\frac{E_{1}^{s}}{E_{2}^{s}} \rho\left(b_{1}^{\mathrm{s}}-\hat{b}_{1}^{\mathrm{s}}\right)\right\}\right)}\right.\right.
\end{aligned}
$$

since $z_{21}^{\mathbf{s}}=\sqrt{E_{1}^{s}} \rho$ and $\mathbf{Z}_{\mathbf{s}}^{(2)}=\left[z_{22}^{\mathbf{s}}\right]=\left[\sqrt{E_{2}^{s}}\right]$. Then assuming equally likely transmitted bits, the BER of user 2 is

$$
\begin{aligned}
& P_{e, 2}\left(N_{0}\right)=P\left(\hat{b}_{2}^{\mathrm{s}} \neq b_{2}^{\mathrm{s}}\right) \\
& =P\left(\hat{b}_{2}^{\mathrm{s}}=1 \mid b_{2}^{\mathrm{s}}=-1\right) \text { or }=P\left(\hat{b}_{2}^{\mathrm{s}}=-1 \mid b_{2}^{\mathrm{s}}=1\right) \quad \text { (due to symmetry) } \\
& =P\left(\hat{b}_{2}^{\mathrm{s}}=1 \mid b_{2}^{\mathrm{s}}=-1\right) \\
& =P\left(\hat{b}_{2}^{\mathrm{s}}=1 \mid\left(b_{2}^{\mathrm{s}}=-1, b_{1}^{\mathrm{s}}=1\right)\right) \cdot P\left(b_{1}^{\mathrm{s}}=1\right)+P\left(\hat{b}_{2}^{\mathrm{s}}=1 \mid\left(b_{2}^{\mathrm{s}}=-1, b_{1}^{\mathrm{s}}=-1\right)\right) \cdot P\left(b_{1}^{\mathrm{s}}=-1\right) \\
& =\frac{1}{2} P\left(\hat{b}_{2}^{\mathrm{s}}=1 \mid\left(b_{2}^{\mathrm{s}}=-1, b_{1}^{\mathrm{s}}=1\right)\right)+\frac{1}{2} P\left(\hat{b}_{2}^{\mathrm{s}}=1 \mid\left(b_{2}^{\mathrm{s}}=-1, b_{1}^{\mathrm{s}}=-1\right)\right)
\end{aligned}
$$


where $P\left(\hat{b}_{2}^{\mathrm{s}}=1 \mid\left(b_{2}^{\mathrm{s}}=-1, b_{1}^{\mathrm{s}}=1\right)\right)$ can be obtained as:

$$
\begin{aligned}
P & \left(\hat{b}_{2}^{\mathrm{s}}=1 \mid\left(b_{2}^{\mathrm{s}}=-1, b_{1}^{\mathrm{s}}=1\right)\right) \\
= & P\left(\hat{b}_{2}^{\mathrm{s}}=1 \mid\left(b_{2}^{\mathrm{s}}=-1, b_{1}^{\mathrm{s}}=1, \hat{b}_{1}^{\mathrm{s}}=1\right)\right) \cdot P\left(\hat{b}_{1}^{\mathrm{s}}=1 \mid\left(b_{2}^{\mathrm{s}}=-1, b_{1}^{\mathrm{s}}=1\right)\right) \\
& +P\left(\hat{b}_{2}^{\mathrm{s}}=1 \mid\left(b_{2}^{\mathrm{s}}=-1, b_{1}^{\mathrm{s}}=1, \hat{b}_{1}^{\mathrm{s}}=-1\right)\right) \cdot P\left(\hat{b}_{1}^{\mathrm{s}}=-1 \mid\left(b_{2}^{\mathrm{s}}=-1, b_{1}^{\mathrm{s}}=1\right)\right) \\
= & P\left(\hat{b}_{2}^{\mathrm{s}}=1 \mid\left(b_{2}^{\mathrm{s}}=-1, b_{1}^{\mathrm{s}}=1, \hat{b}_{1}^{\mathrm{s}}=1\right)\right) \cdot P\left(\hat{b}_{1}^{\mathrm{s}}=1 \mid b_{1}^{\mathrm{s}}=1\right) \\
& +P\left(\hat{b}_{2}^{\mathrm{s}}=1 \mid\left(b_{2}^{\mathrm{s}}=-1, b_{1}^{\mathrm{s}}=1, \hat{b}_{1}^{\mathrm{s}}=-1\right)\right) \cdot P\left(\hat{b}_{1}^{\mathrm{s}}=-1 \mid b_{1}^{\mathrm{s}}=1\right) \\
= & P\left(\hat{b}_{2}^{\mathrm{s}}=1 \mid\left(b_{2}^{\mathrm{s}}=-1, b_{1}^{\mathrm{s}}=1, \hat{b}_{1}^{\mathrm{s}}=1\right)\right) \cdot\left(1-P\left(\hat{b}_{1}^{\mathrm{s}}=-1 \mid b_{1}^{\mathrm{s}}=1\right)\right) \\
& +P\left(\hat{b}_{2}^{\mathrm{s}}=1 \mid\left(b_{2}^{\mathrm{s}}=-1, b_{1}^{\mathrm{s}}=1, \hat{b}_{1}^{\mathrm{s}}=-1\right)\right) \cdot P\left(\hat{b}_{1}^{\mathrm{s}}=-1 \mid b_{1}^{\mathrm{s}}=1\right) \\
= & P\left(-1+\frac{1}{\sqrt{E_{2}^{s}}} \Re\left\{n_{2}^{\mathrm{s}}\right\}+\rho \sqrt{\frac{E_{1}^{s}}{E_{2}^{s}}}(1-1)>0\right) \cdot\left(1-Q\left(\sqrt{\frac{2 E_{1}^{s}\left(1-\rho^{2}\right)}{N_{0}}}\right)\right) \\
& +P\left(-1+\frac{1}{\sqrt{E_{2}^{s}}} \Re\left\{n_{2}^{\mathrm{s}}\right\}+\rho \sqrt{\frac{E_{1}^{s}}{E_{2}^{s}}}(1-(-1))>0\right) \cdot Q\left(\sqrt{\frac{2 E_{1}^{s}\left(1-\rho^{2}\right)}{N_{0}}}\right) \\
= & P\left(\Re\left\{n_{2}^{\mathrm{s}}\right\}>\sqrt{E_{2}^{s}}\right) \cdot\left(1-Q\left(\sqrt{\frac{2 E_{1}^{s}\left(1-\rho^{2}\right)}{N_{0}}}\right)\right) \\
& +P\left(\Re\left\{n_{2}^{\mathrm{s}}\right\}>\sqrt{E_{2}^{s}}-2 \rho \sqrt{E_{1}^{s}}\right) \cdot Q\left(\sqrt{\frac{2 E_{1}^{s}\left(1-\rho^{2}\right)}{N_{0}}}\right) \\
& +Q\left(\frac{\sqrt{2 E_{2}^{s}}-2 \rho \sqrt{2 E_{1}^{s}}}{\sqrt{N_{0}}}\right) \cdot Q\left(\sqrt{\frac{2 E_{1}^{s}\left(1-\rho^{2}\right)}{N_{0}}}\right) \\
& Q\left(\sqrt{\frac{2 E_{2}^{s}}{N_{0}}}\right) \cdot\left(1-Q\left(\sqrt{\frac{2 E_{1}^{s}\left(1-\rho^{2}\right)}{N_{0}}}\right)\right) \\
& (1)
\end{aligned}
$$

where we have used (D.4) to evaluate $P\left(\hat{b}_{2}^{\mathrm{s}}=1 \mid\left(b_{2}^{\mathrm{s}}=-1, b_{1}^{\mathrm{s}}=1, \hat{b}_{1}^{\mathrm{s}}=i\right)\right), E\left[\left(\Re\left\{n_{2}^{\mathrm{s}}\right\}\right)^{2}\right]=$ 
$E\left[\left|n_{2}^{\mathrm{s}}\right|^{2} / 2\right]=\left(N_{0} / 2\right)\left[\boldsymbol{R}_{\mathrm{s}}\right]_{22}=\left(N_{0} / 2\right)$ and $P\left(\hat{b}_{1}^{\mathrm{s}}=-1 \mid b_{1}^{\mathrm{s}}=1\right)$ can be evaluated as

$$
\begin{aligned}
P\left(\hat{b}_{1}^{\mathrm{s}}=-1 \mid b_{1}^{\mathrm{s}}=1\right) & =P\left(1+\Re\left\{\left[\left(\boldsymbol{R}_{\mathrm{s}} \boldsymbol{E}_{\mathrm{s}}\right)^{-1} \vec{n}^{\mathrm{s}}\right]_{1}\right\}<0\right)=P\left(-\Re\left\{\left[\left(\boldsymbol{R}_{\mathrm{s}} \boldsymbol{E}_{\mathrm{s}}\right)^{-1} \vec{n}^{\mathrm{s}}\right]_{1}>1\right\}\right) \\
& =Q\left(\frac{1}{\sqrt{\frac{N_{0}}{2} \frac{1}{E_{1}^{\mathrm{s}}}\left[\boldsymbol{R}_{\mathrm{s}}^{-1}\right]_{11}}}\right)=Q\left(\sqrt{\frac{2 E_{1}^{s}\left(1-\rho^{2}\right)}{N_{0}}}\right)
\end{aligned}
$$

since

$$
\begin{aligned}
\hat{b}_{1}^{\mathrm{s}} & =\operatorname{sgn}\left(\Re\left\{\left[\left(\boldsymbol{R}_{\mathrm{s}} \boldsymbol{E}_{\mathrm{s}}\right)^{-1} \vec{y}^{\mathrm{s}}\right]_{1}\right\}\right)=\operatorname{sgn}\left(\Re\left\{\left[\vec{b}^{\mathrm{s}}+\left(\boldsymbol{R}_{\mathrm{s}} \boldsymbol{E}_{\mathrm{s}}\right)^{-1} \vec{n}^{\mathrm{s}}\right]_{1}\right\}\right) \\
& =\operatorname{sgn}\left(\Re\left\{b_{1}^{\mathrm{s}}+\left[\left(\boldsymbol{R}_{\mathrm{s}} \boldsymbol{E}_{\mathrm{s}}\right)^{-1} \vec{n}^{\mathrm{s}}\right]_{1}\right\}\right)=\operatorname{sgn}\left(b_{1}^{\mathrm{s}}+\Re\left\{\left[\left(\boldsymbol{R}_{\mathrm{s}} \boldsymbol{E}_{\mathrm{s}}\right)^{-1} \vec{n}^{\mathrm{s}}\right]_{1}\right\}\right)
\end{aligned}
$$

and $\Re\left\{\left[\left(\boldsymbol{R}_{\mathrm{s}} \boldsymbol{E}_{\mathrm{s}}\right)^{-1} \vec{n}^{\mathrm{s}}\right]_{1}\right\}$ is zero mean and has the same variance as $\left[\left(\boldsymbol{R}_{\mathrm{s}} \boldsymbol{E}_{\mathrm{s}}\right)^{-1} \vec{n}^{\mathrm{s}}\right]_{1}$ given by

$$
\begin{aligned}
& E\left[\left|\left[\left(\boldsymbol{R}_{\mathrm{s}} \boldsymbol{E}_{\mathrm{s}}\right)^{-1} \vec{n}^{\mathrm{s}}\right]_{1}\right|^{2} / 2\right]=\left[E\left[\left(\boldsymbol{R}_{\mathrm{s}} \boldsymbol{E}_{\mathrm{s}}\right)^{-1} \vec{n}^{\mathrm{s}}\left(\left(\boldsymbol{R}_{\mathrm{s}} \boldsymbol{E}_{\mathrm{s}}\right)^{-1} \vec{n}^{\mathrm{s}}\right)^{\dagger} / 2\right]\right]_{11} \\
& =\left[\left(\boldsymbol{E}_{\mathrm{s}}\right)^{-1}\left(\boldsymbol{R}_{\mathrm{s}}\right)^{-1} E\left[\vec{n}^{\mathrm{s}} \vec{n}^{\mathrm{s} \dagger} / 2\right]\left(\boldsymbol{R}_{\mathrm{s}} \boldsymbol{E}_{\mathrm{s}}\right)^{-1 \dagger}\right]_{11}=\left[\left(\boldsymbol{E}_{\mathrm{s}}\right)^{-1}\left(\boldsymbol{R}_{\mathrm{s}}\right)^{-1} \frac{N_{0}}{2} \boldsymbol{R}_{\mathrm{s}}\left(\boldsymbol{R}_{\mathrm{s}} \boldsymbol{E}_{\mathrm{s}}\right)^{-1 \dagger}\right]_{11} \\
& =\left[\frac{N_{0}}{2}\left(\boldsymbol{E}_{\mathrm{s}}\right)^{-1}\left(\boldsymbol{R}_{\mathrm{s}} \boldsymbol{E}_{\mathrm{s}}\right)^{-1 \dagger}\right]_{11}=\frac{N_{0}}{2}\left[\left(\boldsymbol{E}_{\mathrm{s}}\right)^{-1}\right]_{11}\left[\left(\boldsymbol{R}_{\mathrm{s}} \boldsymbol{E}_{\mathrm{s}}\right)^{-1 \dagger}\right]_{11} \\
& =\frac{N_{0}}{2} \frac{1}{\sqrt{E_{1}^{s}}}\left[\boldsymbol{R}_{\mathrm{s}}^{-1 \dagger} \boldsymbol{E}_{\mathrm{s}}^{-1 \dagger}\right]_{11}=\frac{N_{0}}{2} \frac{1}{\sqrt{E_{1}^{s}}}\left[\boldsymbol{R}_{\mathrm{s}}^{-1 \dagger}\right]_{11}\left[\boldsymbol{E}_{\mathrm{s}}^{-1 \dagger}\right]_{11}=\frac{N_{0}}{2} \frac{1}{E_{1}^{s}}\left[\boldsymbol{R}_{\mathrm{s}}^{-1}\right]_{11}=\frac{N_{0}}{2} \frac{1}{E_{1}^{s}} \frac{1}{1-\rho^{2}}
\end{aligned}
$$

recalling that

$$
\boldsymbol{R}_{\mathrm{s}}=\left(\begin{array}{ll}
1 & \rho \\
\rho & 1
\end{array}\right) \quad \rightarrow \quad \boldsymbol{R}_{\mathrm{s}}^{-1}=\frac{1}{1-\rho^{2}}\left(\begin{array}{cc}
1 & -\rho \\
-\rho & 1
\end{array}\right)
$$


Similarly $P\left(\hat{b}_{2}^{\mathrm{s}}=1 \mid\left(b_{2}^{\mathrm{s}}=-1, b_{1}^{\mathrm{s}}=-1\right)\right)$ can be obtained as:

$$
\begin{aligned}
& P\left(\hat{b}_{2}^{\mathrm{s}}=1 \mid\left(b_{2}^{\mathrm{s}}=-1, b_{1}^{\mathrm{s}}=-1\right)\right) \\
& =P\left(\hat{b}_{2}^{\mathrm{s}}=1 \mid\left(b_{2}^{\mathrm{s}}=-1, b_{1}^{\mathrm{s}}=-1, \hat{b}_{1}^{\mathrm{s}}=1\right)\right) \cdot P\left(\hat{b}_{1}^{\mathrm{s}}=1 \mid\left(b_{2}^{\mathrm{s}}=-1, b_{1}^{\mathrm{s}}=-1\right)\right) \\
& +P\left(\hat{b}_{2}^{\mathrm{s}}=1 \mid\left(b_{2}^{\mathrm{s}}=-1, b_{1}^{\mathrm{s}}=-1, \hat{b}_{1}^{\mathrm{s}}=-1\right)\right) \cdot P\left(\hat{b}_{1}^{\mathrm{s}}=-1 \mid\left(b_{2}^{\mathrm{s}}=-1, b_{1}^{\mathrm{s}}=-1\right)\right) \\
& =P\left(\hat{b}_{2}^{\mathrm{s}}=1 \mid\left(b_{2}^{\mathrm{s}}=-1, b_{1}^{\mathrm{s}}=-1, \hat{b}_{1}^{\mathrm{s}}=1\right)\right) \cdot P\left(\hat{b}_{1}^{\mathrm{s}}=1 \mid b_{1}^{\mathrm{s}}=-1\right) \\
& +P\left(\hat{b}_{2}^{\mathrm{s}}=1 \mid\left(b_{2}^{\mathrm{s}}=-1, b_{1}^{\mathrm{s}}=-1, \hat{b}_{1}^{\mathrm{s}}=-1\right)\right) \cdot P\left(\hat{b}_{1}^{\mathrm{s}}=-1 \mid b_{1}^{\mathrm{s}}=-1\right) \\
& =P\left(\hat{b}_{2}^{\mathrm{s}}=1 \mid\left(b_{2}^{\mathrm{s}}=-1, b_{1}^{\mathrm{s}}=-1, \hat{b}_{1}^{\mathrm{s}}=1\right)\right) \cdot P\left(\hat{b}_{1}^{\mathrm{s}}=1 \mid b_{1}^{\mathrm{s}}=-1\right) \\
& +P\left(\hat{b}_{2}^{\mathrm{s}}=1 \mid\left(b_{2}^{\mathrm{s}}=-1, b_{1}^{\mathrm{s}}=-1, \hat{b}_{1}^{\mathrm{s}}=-1\right)\right) \cdot\left(1-P\left(\hat{b}_{1}^{\mathrm{s}}=-1 \mid b_{1}^{\mathrm{s}}=1\right)\right) \\
& =P\left(-1+\frac{1}{\sqrt{E_{2}^{s}}} \Re\left\{n_{2}^{\mathrm{s}}\right\}+\rho \sqrt{\frac{E_{1}^{s}}{E_{2}^{s}}}(-1-1)>0\right) \cdot Q\left(\sqrt{\frac{2 E_{1}^{s}\left(1-\rho^{2}\right)}{N_{0}}}\right) \\
& +P\left(-1+\frac{1}{\sqrt{E_{2}^{s}}} \Re\left\{n_{2}^{\mathrm{s}}\right\}+\rho \sqrt{\frac{E_{1}^{s}}{E_{2}^{s}}}(-1-(-1))>0\right) \cdot\left(1-Q\left(\sqrt{\frac{2 E_{1}^{s}\left(1-\rho^{2}\right)}{N_{0}}}\right)\right) \\
& =P\left(\Re\left\{n_{2}^{\mathrm{s}}\right\}>\sqrt{E_{2}^{s}}+2 \rho \sqrt{E_{1}^{s}}\right) \cdot Q\left(\sqrt{\frac{2 E_{1}^{s}\left(1-\rho^{2}\right)}{N_{0}}}\right) \\
& +P\left(\Re\left\{n_{2}^{\mathrm{s}}\right\}>\sqrt{E_{2}^{s}}\right) \cdot\left(1-Q\left(\sqrt{\frac{2 E_{1}^{s}\left(1-\rho^{2}\right)}{N_{0}}}\right)\right) \\
& =Q\left(\frac{\sqrt{2 E_{2}^{s}}+2 \rho \sqrt{2 E_{1}^{s}}}{\sqrt{N_{0}}}\right) \cdot Q\left(\sqrt{\frac{2 E_{1}^{s}\left(1-\rho^{2}\right)}{N_{0}}}\right) \\
& +Q\left(\sqrt{\frac{2 E_{2}^{s}}{N_{0}}}\right) \cdot\left(1-Q\left(\sqrt{\frac{2 E_{1}^{s}\left(1-\rho^{2}\right)}{N_{0}}}\right)\right)
\end{aligned}
$$


Therefore substituting (D.6) and (D.7) into (D.5) yields

$$
\begin{aligned}
& P_{e, 2}\left(N_{0}\right)=Q\left(\sqrt{\frac{2 E_{2}^{s}}{N_{0}}}\right) \times\left(1-Q\left(\sqrt{\frac{2 E_{1}^{s}\left(1-\rho^{2}\right)}{N_{0}}}\right)\right) \\
& \quad+\frac{1}{2} Q\left(\sqrt{\frac{2 E_{1}^{s}\left(1-\rho^{2}\right)}{N_{0}}}\right) \times\left[Q\left(\frac{\sqrt{2 E_{2}^{s}}-2 \rho \sqrt{2 E_{1}^{s}}}{\sqrt{N_{0}}}\right)+Q\left(\frac{\sqrt{2 E_{2}^{s}}+2 \rho \sqrt{2 E_{1}^{s}}}{\sqrt{N_{0}}}\right)\right] \\
& =P_{e, 2}^{a}\left(N_{0}\right)+P_{e, 2}^{b}\left(N_{0}\right)
\end{aligned}
$$

where

$$
\left\{\begin{array}{l}
P_{e, 2}^{a}\left(N_{0}\right)=Q\left(\sqrt{\frac{2 E_{2}^{s}}{N_{0}}}\right) \times\left(1-Q\left(\sqrt{\frac{2 E_{1}^{s}\left(1-\rho^{2}\right)}{N_{0}}}\right)\right) \\
P_{e, 2}^{b}\left(N_{0}\right)=\frac{1}{2} Q\left(\sqrt{\frac{2 E_{1}^{s}\left(1-\rho^{2}\right)}{N_{0}}}\right) \times\left[Q\left(\frac{\sqrt{2 E_{2}^{s}}-2 \rho \sqrt{2 E_{1}^{s}}}{\sqrt{N_{0}}}\right)+Q\left(\frac{\sqrt{2 E_{2}^{s}}+2 \rho \sqrt{2 E_{1}^{s}}}{\sqrt{N_{0}}}\right)\right]
\end{array}\right.
$$

it can be shown that

$$
P_{e, 2}^{a}\left(N_{0}\right)+P_{e, 2}^{b}\left(N_{0}\right)=P_{2}\left(N_{0}\right) \geq \max \left\{P_{e, 2}^{a}\left(N_{0}\right), P_{e, 2}^{b}\left(N_{0}\right)\right\}
$$

where $P_{e, 2}^{a}\left(N_{0}\right)+P_{e, 2}^{b}\left(N_{0}\right)$ can be treated as the upper bound of $P_{2}\left(N_{0}\right)$ and $\max \left\{P_{e, 2}^{a}\left(N_{0}\right), P_{e, 2}^{b}\left(N_{0}\right)\right\}$ as the lower bound. The term $P_{e, 2}^{a}\left(N_{0}\right)$ is actually the BER of user 2 when the decision on user 1 at the first stage is correct and the term $P_{e, 2}^{b}\left(N_{0}\right)$ is the BER of user 2 when the decision on user 1 is wrong. As $N_{0}$ approaches to zero, one of the terms will dominate and the ratio of the upper and lower bound approaches to 1 . Therefore,

$$
\lim _{N_{0} \rightarrow 0} \frac{P_{e, 2}^{a}\left(N_{0}\right)+P_{e, 2}^{b}\left(N_{0}\right)}{\max \left\{P_{e, 2}^{a}\left(N_{0}\right), P_{e, 2}^{b}\left(N_{0}\right)\right\}}=1
$$


Therefore $\eta_{2}$ can be obtained through $\max \left\{P_{e, 2}^{a}\left(N_{0}\right), P_{e, 2}^{b}\left(N_{0}\right)\right\}$ or equivalently

$$
\eta_{2}=\min \left\{\eta_{2}^{a}, \eta_{2}^{b}\right\}
$$

where $\eta_{2}^{a}, \eta_{2}^{b}$ correspond to $P_{e, 2}^{a}\left(N_{0}\right), P_{e, 2}^{b}\left(N_{0}\right)$ respectively according to (5.6-5.7). It is known (referring to equations 3.41 and 3.43 in [2]) that

$$
\lim _{x \rightarrow \infty} \frac{Q(\alpha x)}{Q(\beta x)}= \begin{cases}+\infty, & {[\alpha]^{+}<\beta} \\ 2, & \alpha<\beta=0 \\ 1, & \alpha=\beta \text { or } \max \{\alpha, \beta\}<0 \\ \frac{1}{2}, & \beta<\alpha=0 \\ 0, & {[\beta]^{+}<\alpha}\end{cases}
$$

where $[\alpha]^{+}=\max \{0, \alpha\}$. Using (D.12), for all $r \in\left[0,1\left[\right.\right.$, we have $0 \leq \sqrt{2 r E_{2}^{s}}<\sqrt{2 E_{2}^{s}}$ and

$$
\lim _{N_{0} \rightarrow 0} \frac{Q\left(\sqrt{\frac{2 E_{2}^{s}}{N_{0}}}\right)}{Q\left(\sqrt{\frac{2 r E_{2}^{s}}{N_{0}}}\right)}=0 \rightarrow \lim _{N_{0} \rightarrow 0} \frac{P_{e, 2}^{a}\left(N_{0}\right)}{Q\left(\sqrt{\frac{2 r E_{2}^{s}}{N_{0}}}\right)}=0
$$

therefore,

$$
\eta_{2}^{a}=1
$$

From (5.7)

$$
\eta_{2}^{b}=\frac{1}{E_{2}^{s}} \lim _{N_{0} \rightarrow 0} N_{0} \log \frac{1}{P_{e, 2}^{b}\left(N_{0}\right)}=\eta_{2}^{(b, 1)}+\eta_{2}^{(b, 2)}
$$

where

$$
\eta_{2}^{(b, 1)}=\frac{1}{E_{2}^{s}} \lim _{N_{0} \rightarrow 0} N_{0} \log \frac{1}{Q\left(\sqrt{\frac{2 E_{1}^{s}\left(1-\rho^{2}\right)}{N_{0}}}\right)}=\frac{1}{E_{2}^{s}}\left(\sqrt{E_{1}^{s}\left(1-\rho^{2}\right)}\right)^{2}=\frac{E_{1}^{s}}{E_{2}^{s}}\left(1-\rho^{2}\right)
$$


since $2 \lim _{\sigma \rightarrow 0} \sigma^{2} \log Q(x / \sigma)=-\left([x]^{+}\right)^{2}$, where $[x]^{+}=\max \{0, x\}$.

$$
\begin{aligned}
\eta_{2}^{(b, 2)} & =\frac{1}{E_{2}^{s}} \lim _{N_{0} \rightarrow 0} N_{0} \log \frac{1}{\left[\frac{1}{2} Q\left(\frac{\sqrt{2 E_{2}^{s}}-2 \rho \sqrt{2 E_{1}^{s}}}{\sqrt{N_{0}}}\right)+\frac{1}{2} Q\left(\frac{\sqrt{2 E_{2}^{s}}+2 \rho \sqrt{2 E_{1}^{s}}}{\sqrt{N_{0}}}\right)\right]} \\
& =\sup _{r \in[0,1]}\left\{\lim _{N_{0} \rightarrow 0} \frac{\left[\frac{1}{2} Q\left(\frac{\sqrt{2 E_{2}^{s}}-2 \rho \sqrt{2 E_{1}^{s}}}{\sqrt{N_{0}}}\right)+\frac{1}{2} Q\left(\frac{\sqrt{2 E_{2}^{s}}+2 \rho \sqrt{2 E_{1}^{s}}}{\sqrt{N_{0}}}\right)\right]}{Q\left(\sqrt{\frac{2 r E_{2}^{s}}{N_{0}}}\right)}=0\right\}
\end{aligned}
$$

From (D.12), it is observed that if $\sqrt{E_{2}^{s}} \leq 2|\rho| \sqrt{E_{1}^{s}}$, the term

$$
\left[\frac{1}{2} Q\left(\frac{\sqrt{2 E_{2}^{s}}-2 \rho \sqrt{2 E_{1}^{s}}}{\sqrt{N_{0}}}\right)+\frac{1}{2} Q\left(\frac{\sqrt{2 E_{2}^{s}}+2 \rho \sqrt{2 E_{1}^{s}}}{\sqrt{N_{0}}}\right)\right]
$$

does not approach to zero as $N_{0}$ goes to zero which implies that $\eta_{2}^{(b, 2)}=0$. If $\sqrt{E_{2}^{s}}>$ $2|\rho| \sqrt{E_{1}^{s}}$ then

$$
\lim _{N_{0} \rightarrow 0} \frac{\left[\frac{1}{2} Q\left(\frac{\sqrt{2 E_{2}^{s}}-2 \rho \sqrt{2 E_{1}^{s}}}{\sqrt{N_{0}}}\right)+\frac{1}{2} Q\left(\frac{\sqrt{2 E_{2}^{s}}+2 \rho \sqrt{2 E_{1}^{s}}}{\sqrt{N_{0}}}\right)\right]}{Q\left(\sqrt{\frac{2 r E_{2}^{s}}{N_{0}}}\right)}=0, \text { when } \begin{gathered}
\sqrt{2 r E_{2}^{s}}<\sqrt{2 E_{2}^{s}}-2|\rho| \sqrt{2 E_{1}^{s}} \\
\Rightarrow r<\left(1-2|\rho| \sqrt{\frac{E_{1}^{s}}{E_{2}^{s}}}\right)^{2}
\end{gathered}
$$

which implies that $\eta_{2}^{(b, 2)}=\left(1-2|\rho| \sqrt{\frac{E_{1}^{s}}{E_{2}^{s}}}\right)^{2}$, therefore,

$$
\eta_{2}^{(b, 2)}=\left(\max \left\{0,1-2|\rho| \sqrt{\frac{E_{1}^{s}}{E_{2}^{s}}}\right\}\right)^{2}
$$

Combining (D.11), (D.13), (D.14), (D.15) and (D.17), it is obtained that

$$
\eta_{2}=\min \left\{1, \frac{E_{1}^{s}}{E_{2}^{s}}\left(1-\rho^{2}\right)+\left(\max \left\{0,1-2|\rho| \sqrt{\frac{E_{1}^{s}}{E_{2}^{s}}}\right\}\right)^{2}\right\}
$$




\section{Appendix E}

\section{AME Performance Analysis of}

\section{DBSIC for Rayleigh Channels}

Considering a two-user system over a single-path Rayleigh fading channel, the matched filter outputs (5.3) reduces to

$$
\vec{y}^{\mathrm{s}}=\left(\begin{array}{c}
y_{1}^{\mathrm{s}} \\
y_{2}^{\mathrm{s}}
\end{array}\right)=\boldsymbol{R}_{\mathrm{s}} \boldsymbol{E}_{\mathrm{s}} \vec{b}^{\mathrm{s}}+\vec{n}^{\mathrm{s}}=\left(\begin{array}{cc}
\rho_{11}^{\mathrm{s}} & \rho_{21}^{\mathrm{s}} \\
\rho_{12}^{\mathrm{s}} & \rho_{22}^{\mathrm{s}}
\end{array}\right)\left(\begin{array}{cc}
\sqrt{E_{1}^{s}} & 0 \\
0 & \sqrt{E_{2}^{s}}
\end{array}\right)\left(\begin{array}{c}
b_{1}^{\mathrm{s}} \\
b_{2}^{\mathrm{s}}
\end{array}\right)+\left(\begin{array}{c}
n_{1}^{\mathrm{s}} \\
n_{2}^{\mathrm{s}}
\end{array}\right)
$$

where

$$
\begin{aligned}
& \rho_{11}^{\mathrm{s}}=\int_{0}^{T_{s}} C_{1}(t) C_{1}^{*}(t) d t=\int_{0}^{T_{s}} \alpha_{1} S_{1}(t) \alpha_{1}^{*} S_{1}^{*}(t) d t=\left|\alpha_{1}\right|^{2} \\
& \rho_{12}^{\mathrm{s}}=\int_{0}^{T_{s}} C_{1}(t) C_{2}^{*}(t) d t=\int_{0}^{T_{s}} \alpha_{1} S_{1}(t) \alpha_{2}^{*} S_{2}^{*}(t) d t=\alpha_{1} \alpha_{2}^{*} \rho \\
& \rho_{21}^{\mathrm{s}}=\int_{0}^{T_{s}} C_{2}(t) C_{1}^{*}(t) d t=\int_{0}^{T_{s}} \alpha_{2} S_{2}(t) \alpha_{1}^{*} S_{1}^{*}(t) d t=\alpha_{1}^{*} \alpha_{2} \rho \\
& \rho_{22}^{\mathrm{s}}=\int_{0}^{T_{s}} C_{2}(t) C_{2}^{*}(t) d t=\int_{0}^{T_{s}} \alpha_{2} S_{2}(t) \alpha_{2}^{*} S_{2}^{*}(t) d t=\left|\alpha_{2}\right|^{2}
\end{aligned}
$$


and where $\rho$ is the cross-correlation between user 1 and user 2 given by (D.2) (assumed to be real for simplicity).

$$
\left\{\begin{array}{l}
y_{1}^{\mathrm{s}}=\sqrt{E_{1}^{s}} \rho_{11}^{\mathrm{s}} b_{1}^{\mathrm{s}}+\sqrt{E_{2}^{s}} \rho_{21}^{\mathrm{s}} b_{2}^{\mathrm{s}}+n_{1}^{\mathrm{s}}=\left|\alpha_{1}\right|^{2} \sqrt{E_{1}^{s}} b_{1}^{\mathrm{s}}+\alpha_{2} \alpha_{1}^{*} \sqrt{E_{2}^{s}} \rho b_{2}^{\mathrm{s}}+n_{1}^{\mathrm{s}} \\
y_{2}^{\mathrm{s}}=\sqrt{E_{1}^{s}} \rho_{12}^{\mathrm{s}} b_{1}^{\mathrm{s}}+\sqrt{E_{2}^{s}} \rho_{22}^{\mathrm{s}} b_{2}^{\mathrm{s}}+n_{2}^{\mathrm{s}}=\alpha_{1} \alpha_{2}^{*} \sqrt{E_{1}^{s}} \rho b_{1}^{\mathrm{s}}+\left|\alpha_{2}\right|^{2} \sqrt{E_{2}^{s}} b_{2}^{\mathrm{s}}+n_{2}^{\mathrm{s}}
\end{array}\right.
$$

For the Rayleigh fading channel, $\left|\alpha_{k}\right|$ has a Rayleigh probability distribution:

$$
P_{R}(r)=r e^{-r^{2} / 2}, \quad r \geq 0 \quad\left(r=\left|\alpha_{k}\right|\right)
$$

$$
\begin{aligned}
& P_{e, 1 \mid \alpha_{1}}\left(N_{0}\right)=P\left(\hat{b}_{1}^{\mathrm{s}} \neq b_{1}^{\mathrm{s}} \mid \alpha_{1}\right) \\
& =P\left(\hat{b}_{1}^{\mathrm{s}}=-1 \mid b_{1}^{\mathrm{s}}=1, \alpha_{1}\right) \cdot P\left(b_{1}^{\mathrm{s}}=1 \mid \alpha_{1}\right)+P\left(\hat{b}_{1}^{\mathrm{s}}=1 \mid b_{1}^{\mathrm{s}}=-1, \alpha_{1}\right) \cdot P\left(b_{1}^{\mathrm{s}}=-1 \mid \alpha_{1}\right) \\
& =\frac{1}{2} P\left(\hat{b}_{1}^{\mathrm{s}}=-1 \mid b_{1}^{\mathrm{s}}=1, \alpha_{1}\right)+\frac{1}{2} P\left(\hat{b}_{1}^{\mathrm{s}}=1 \mid b_{1}^{\mathrm{s}}=-1, \alpha_{1}\right) \\
& =P\left(\hat{b}_{1}^{\mathrm{s}}=-1 \mid b_{1}^{\mathrm{s}}=1, \alpha_{1}\right) \text { or }=P\left(\hat{b}_{1}^{\mathrm{s}}=-1 \mid b_{1}^{\mathrm{s}}=1, \alpha_{1}\right) \quad \text { (due to symmetry) }
\end{aligned}
$$

Using similar techniques as for the AWGN channel, we obtain

$$
\begin{aligned}
P\left(\hat{b}_{1}^{\mathrm{s}}=-1 \mid b_{1}^{\mathrm{s}}=1, \alpha_{1}\right) & =P\left(1+\Re\left\{\left[\left(\boldsymbol{R}_{\mathrm{s}} \boldsymbol{E}_{\mathrm{s}}\right)^{-1} \vec{n}^{\mathrm{s}}\right]_{1}\right\}<0 \mid \alpha_{1}\right) \\
& =P\left(-\Re\left\{\left[\left(\boldsymbol{R}_{\mathrm{s}} \boldsymbol{E}_{\mathrm{s}}\right)^{-1} \vec{n}^{\mathrm{s}}\right]_{1}\right\}>1 \mid \alpha_{1}\right) \\
& =Q\left(\frac{1}{\sqrt{\frac{N_{0}}{2} \frac{1}{E_{1}^{s}}\left[\boldsymbol{R}_{\mathrm{s}}^{-1}\right]_{11}}}\right)=Q\left(\sqrt{\frac{2 E_{1}^{s}\left(1-\rho^{2}\right)}{N_{0}}}\left|\alpha_{1}\right|\right)
\end{aligned}
$$

since

$$
\boldsymbol{R}_{\mathrm{s}}=\left(\begin{array}{cc}
\rho_{11}^{\mathrm{s}} & \rho_{21}^{\mathrm{s}} \\
\rho_{12}^{\mathrm{S}} & \rho_{22}^{\mathrm{s}}
\end{array}\right)=\left(\begin{array}{cc}
\left|\alpha_{1}\right|^{2} & \alpha_{1}^{*} \alpha_{2} \rho \\
\alpha_{1} \alpha_{2}^{*} \rho & \left|\alpha_{2}\right|^{2}
\end{array}\right) \quad \rightarrow \quad \boldsymbol{R}_{\mathrm{s}}^{-1}=\left(\begin{array}{cc}
\frac{\left|\alpha_{2}\right|^{2}}{\left|\alpha_{1} \alpha_{2}\right|^{2}\left(1-\rho^{2}\right)} & \frac{-\alpha_{1}^{*} \alpha_{2} \rho}{\left|\alpha_{1} \alpha_{2}\right|^{2}\left(1-\rho^{2}\right)} \\
\frac{-\alpha_{1} \alpha_{2}^{*} \rho}{\left|\alpha_{1} \alpha_{2}\right|^{2}\left(1-\rho^{2}\right)} & \frac{\left|\alpha_{1}\right|^{2}}{\left|\alpha_{1} \alpha_{2}\right|^{2}\left(1-\rho^{2}\right)}
\end{array}\right)
$$




$$
\begin{aligned}
P_{e, 1}\left(N_{0}\right) & =E\left[P_{e, 1 \mid \alpha_{1}}\left(N_{0}\right)\right]=\int_{0}^{+\infty} P_{R}(r) P_{e, 1 \mid \alpha_{1}}\left(N_{0}\right) d r \\
& =\int_{0}^{+\infty} r e^{-r^{2} / 2} Q\left(\sqrt{\frac{2 E_{1}^{s}\left(1-\rho^{2}\right)}{N_{0}}} r\right) d r \\
& =\frac{1}{2}\left[1-\frac{\sqrt{\frac{2 E_{1}^{s}\left(1-\rho^{2}\right)}{N_{0}}}}{\sqrt{1+\frac{2 E_{1}^{s}\left(1-\rho^{2}\right)}{N_{0}}}}\right]=\frac{1}{2}\left(1-\frac{1}{\sqrt{1+\frac{N_{0} / 2}{E_{1}^{s}\left(1-\rho^{2}\right)}}}\right)
\end{aligned}
$$

where we have used

$$
\begin{aligned}
& \int_{0}^{+\infty} r e^{-r^{2} / 2} Q(\alpha r) d r=\left[-e^{-r^{2} / 2} Q(\alpha r)\right]_{0}^{\infty}-\int_{0}^{+\infty}-e^{-r^{2} / 2}(-1) \frac{\alpha}{\sqrt{2 \pi}} e^{-\alpha^{2} r^{2} / 2} d r \\
& =\frac{1}{2}-\frac{\alpha \sqrt{\frac{1}{1+\alpha^{2}}}}{\sqrt{2 \pi \frac{1}{1+\alpha^{2}}}} \int_{0}^{+\infty} e^{-\frac{r^{2}}{2 \frac{1}{1+\alpha^{2}}}} d r=\frac{1}{2}-\frac{1}{2} \frac{\alpha}{\sqrt{1+\alpha^{2}}}=\frac{1}{2}\left[1-\frac{\alpha}{\sqrt{1+\alpha^{2}}}\right] \quad \alpha \geq 0
\end{aligned}
$$

From (5.19)

$$
\begin{aligned}
\eta_{1} & =\lim _{\sigma \rightarrow 0} \frac{\sigma^{2}}{4 E_{1}^{s} P_{e, 1}(\sigma)}=\lim _{N_{0} \rightarrow 0} \frac{N_{0} / 2}{4 E_{1}^{s} P_{e, 1}\left(N_{0}\right)}=\lim _{N_{0} \rightarrow 0} \frac{N_{0} / 2}{4 E_{1}^{s} \frac{1}{2}\left(1-\frac{1}{\sqrt{1+\frac{N_{0} / 2}{E_{1}^{s}\left(1-\rho^{2}\right)}}}\right)} \\
& =\lim _{N_{0} \rightarrow 0} \frac{N_{0} / 2}{4 E_{1}^{s} \frac{1}{2}\left(1-\left(1-\frac{1}{2} \frac{N_{0} / 2}{E_{1}^{s}\left(1-\rho^{2}\right)}\right)\right)}=\lim _{N_{0} \rightarrow 0} \frac{N_{0} / 2}{4 E_{1}^{s} \frac{1}{2} \frac{1}{2} \frac{N_{0} / 2}{E_{1}^{s}\left(1-\rho^{2}\right)}}=1-\rho^{2}
\end{aligned}
$$

For DBSIC,

$$
\begin{aligned}
y_{2}^{\mathrm{s}(2)} & =y_{2}^{\mathrm{s}(1)}-z_{21}^{\mathrm{s}} \hat{b}_{1}^{\mathrm{s}}=y_{2}^{\mathrm{s}(1)}-\left[\mathbf{Z}_{\mathrm{s}}\right]_{21} \hat{b}_{1}^{\mathrm{s}}=y_{2}^{\mathrm{s}(1)}-\left[\boldsymbol{R}_{\mathrm{s}} \boldsymbol{E}_{\mathrm{s}}\right]_{21} \hat{b}_{1}^{\mathrm{s}}=y_{2}^{\mathrm{s}(1)}-\rho_{12}^{\mathrm{s}} \sqrt{E_{1}^{\mathrm{s}}} \hat{b}_{1}^{\mathrm{s}} \\
& =y_{2}^{\mathrm{s}(1)}-\alpha_{1} \alpha_{2}^{*} \rho \sqrt{E_{1}^{\mathrm{s}}} \hat{b}_{1}^{\mathrm{s}}=y_{2}^{\mathrm{s}}-\alpha_{1} \alpha_{2}^{*} \rho \sqrt{E_{1}^{\mathrm{s}}} \hat{b}_{1}^{\mathrm{s}} \\
& =\alpha_{1} \alpha_{2}^{*} \sqrt{E_{1}^{s}} \rho b_{1}^{\mathrm{s}}+\left|\alpha_{2}\right|^{2} \sqrt{E_{2}^{\mathrm{s}}} b_{2}^{\mathrm{s}}+n_{2}^{\mathrm{s}}-\alpha_{1} \alpha_{2}^{*} \rho \sqrt{E_{1}^{s}} \hat{b}_{1}^{\mathrm{s}} \quad \text { using }(\mathrm{E} .1) \\
& =\left|\alpha_{2}\right|^{2} \sqrt{E_{2}^{s}} b_{2}^{\mathrm{s}}+n_{2}^{\mathrm{s}}+\alpha_{1} \alpha_{2}^{*} \sqrt{E_{1}^{s}} \rho\left(b_{1}^{\mathrm{s}}-\hat{b}_{1}^{\mathrm{s}}\right)
\end{aligned}
$$




$$
\begin{aligned}
\hat{b}_{2}^{\mathrm{s}} & =\operatorname{sgn}\left(\Re\left\{\left[\left(\mathbf{Z}_{\mathrm{s}}^{(2)}\right)^{-1} \vec{y}^{\mathrm{s}(2)}\right]_{1}\right\}\right) \\
& =\operatorname{sgn}\left(\Re\left\{b_{2}^{\mathrm{s}}+\frac{1}{\left|\alpha_{2}\right|^{2} \sqrt{E_{2}^{s}}} n_{2}^{\mathrm{s}}+\frac{\alpha_{1}}{\alpha_{2}} \sqrt{\frac{E_{1}^{s}}{E_{2}^{s}}} \rho\left(b_{1}^{\mathrm{s}}-\hat{b}_{1}^{\mathrm{s}}\right)\right\}\right)
\end{aligned}
$$

since $z_{21}^{\mathrm{s}}=\alpha_{1} \alpha_{2}^{*} \sqrt{E_{1}^{\mathrm{s}}} \rho$ and $\mathbf{Z}_{\mathrm{s}}^{(2)}=\left[z_{22}^{\mathrm{s}}\right]=\left[\rho_{22}^{\mathrm{s}} \sqrt{E_{2}^{s}}\right]=\left[\left|\alpha_{2}\right|^{2} \sqrt{E_{2}^{s}}\right]$. Assuming equally likely transmitted bits, the BER of user 2 is

$$
\begin{aligned}
& P_{e, 2 \mid \alpha_{1}, \alpha_{2}}\left(N_{0}\right)=P\left(\hat{b}_{2}^{\mathrm{s}} \neq b_{2}^{\mathrm{s}} \mid \alpha_{1}, \alpha_{2}\right) \\
&= P\left(\hat{b}_{2}^{\mathrm{s}}=1 \mid b_{2}^{\mathrm{s}}=-1, \alpha_{1}, \alpha_{2}\right) \text { or }=P\left(\hat{b}_{2}^{\mathrm{s}}=-1 \mid b_{2}^{\mathrm{s}}=1, \alpha_{1}, \alpha_{2}\right) \quad \text { (due to symmetry) } \\
&= P\left(\hat{b}_{2}^{\mathrm{s}}=1 \mid b_{2}^{\mathrm{s}}=-1, \alpha_{1}, \alpha_{2}\right) \\
&= P\left(\hat{b}_{2}^{\mathrm{s}}=1 \mid\left(b_{2}^{\mathrm{s}}=-1, b_{1}^{\mathrm{s}}=1\right), \alpha_{1}, \alpha_{2}\right) \cdot P\left(b_{1}^{\mathrm{s}}=1 \mid \alpha_{1}, \alpha_{2}\right) \\
&+P\left(\hat{b}_{2}^{\mathrm{s}}=1 \mid\left(b_{2}^{\mathrm{s}}=-1, b_{1}^{\mathrm{s}}=-1\right), \alpha_{1}, \alpha_{2}\right) \cdot P\left(b_{1}^{\mathrm{s}}=-1 \mid \alpha_{1}, \alpha_{2}\right) \\
&= \frac{1}{2} P\left(\hat{b}_{2}^{\mathrm{s}}=1 \mid\left(b_{2}^{\mathrm{s}}=-1, b_{1}^{\mathrm{s}}=1\right), \alpha_{1}, \alpha_{2}\right)+\frac{1}{2} P\left(\hat{b}_{2}^{\mathrm{s}}=1 \mid\left(b_{2}^{\mathrm{s}}=-1, b_{1}^{\mathrm{s}}=-1\right), \alpha_{1}, \alpha_{2}\right)
\end{aligned}
$$

where $P\left(\hat{b}_{2}^{\mathrm{s}}=1 \mid\left(b_{2}^{\mathrm{s}}=-1, b_{1}^{\mathrm{s}}=1\right), \alpha_{1}, \alpha_{2}\right)$ can be obtained as:

$$
\begin{aligned}
P\left(\hat{b}_{2}^{\mathrm{s}}=1 \mid\left(b_{2}^{\mathrm{s}}=-1, b_{1}^{\mathrm{s}}=1\right), \alpha_{1}, \alpha_{2}\right) \\
=P\left(\hat{b}_{2}^{\mathrm{s}}=1 \mid\left(b_{2}^{\mathrm{s}}=-1, b_{1}^{\mathrm{s}}=1, \hat{b}_{1}^{\mathrm{s}}=1\right), \alpha_{1}, \alpha_{2}\right) \cdot P\left(\hat{b}_{1}^{\mathrm{s}}=1 \mid\left(b_{2}^{\mathrm{s}}=-1, b_{1}^{\mathrm{s}}=1\right), \alpha_{1}, \alpha_{2}\right) \\
\quad+P\left(\hat{b}_{2}^{\mathrm{s}}=1 \mid\left(b_{2}^{\mathrm{s}}=-1, b_{1}^{\mathrm{s}}=1, \hat{b}_{1}^{\mathrm{s}}=-1\right), \alpha_{1}, \alpha_{2}\right) \cdot P\left(\hat{b}_{1}^{\mathrm{s}}=-1 \mid\left(b_{2}^{\mathrm{s}}=-1, b_{1}^{\mathrm{s}}=1\right), \alpha_{1}, \alpha_{2}\right) \\
=P\left(\hat{b}_{2}^{\mathrm{s}}=1 \mid\left(b_{2}^{\mathrm{s}}=-1, b_{1}^{\mathrm{s}}=1, \hat{b}_{1}^{\mathrm{s}}=1\right), \alpha_{1}, \alpha_{2}\right) \cdot P\left(\hat{b}_{1}^{\mathrm{s}}=1 \mid b_{1}^{\mathrm{s}}=1, \alpha_{1}, \alpha_{2}\right) \\
\quad+P\left(\hat{b}_{2}^{\mathrm{s}}=1 \mid\left(b_{2}^{\mathrm{s}}=-1, b_{1}^{\mathrm{s}}=1, \hat{b}_{1}^{\mathrm{s}}=-1\right), \alpha_{1}, \alpha_{2}\right) \cdot P\left(\hat{b}_{1}^{\mathrm{s}}=-1 \mid b_{1}^{\mathrm{s}}=1, \alpha_{1}, \alpha_{2}\right) \\
=P\left(\hat{b}_{2}^{\mathrm{s}}=1 \mid\left(b_{2}^{\mathrm{s}}=-1, b_{1}^{\mathrm{s}}=1, \hat{b}_{1}^{\mathrm{s}}=1\right), \alpha_{1}, \alpha_{2}\right) \cdot\left(1-P\left(\hat{b}_{1}^{\mathrm{s}}=-1 \mid b_{1}^{\mathrm{s}}=1, \alpha_{1}, \alpha_{2}\right)\right) \\
\quad+P\left(\hat{b}_{2}^{\mathrm{s}}=1 \mid\left(b_{2}^{\mathrm{s}}=-1, b_{1}^{\mathrm{s}}=1, \hat{b}_{1}^{\mathrm{s}}=-1\right), \alpha_{1}, \alpha_{2}\right) \cdot P\left(\hat{b}_{1}^{\mathrm{s}}=-1 \mid b_{1}^{\mathrm{s}}=1, \alpha_{1}, \alpha_{2}\right)
\end{aligned}
$$




$$
\begin{aligned}
& =P\left(-1+\frac{1}{\left|\alpha_{2}\right|^{2} \sqrt{E_{2}^{s}}} \Re\left\{n_{2}^{\mathrm{s}}\right\}+\Re\left\{\frac{\alpha_{1}}{\alpha_{2}}\right\} \rho \sqrt{\frac{E_{1}^{s}}{E_{2}^{s}}}(1-1)>0 \mid \alpha_{1}, \alpha_{2}\right) \\
& \cdot\left(1-Q\left(\sqrt{\frac{2 E_{1}^{s}\left(1-\rho^{2}\right)}{N_{0}}}\left|\alpha_{1}\right|\right)\right) \\
& +P\left(-1+\frac{1}{\left|\alpha_{2}\right|^{2} \sqrt{E_{2}^{s}}} \Re\left\{n_{2}^{\mathrm{s}}\right\}+\Re\left\{\frac{\alpha_{1}}{\alpha_{2}}\right\} \rho \sqrt{\frac{E_{1}^{s}}{E_{2}^{s}}}(1-(-1))>0 \mid \alpha_{1}, \alpha_{2}\right) \\
& \cdot\left(\sqrt{\frac{2 E_{1}^{s}\left(1-\rho^{2}\right)}{N_{0}}}\left|\alpha_{1}\right|\right) \\
& =P\left(\Re\left\{n_{2}^{\mathrm{s}}\right\}>\left|\alpha_{2}\right|^{2} \sqrt{E_{2}^{s}} \mid \alpha_{1}, \alpha_{2}\right) \cdot\left(1-Q\left(\sqrt{\frac{2 E_{1}^{s}\left(1-\rho^{2}\right)}{N_{0}}}\left|\alpha_{1}\right|\right)\right) \\
& +P\left(\Re\left\{n_{2}^{\mathrm{s}}\right\}>\left|\alpha_{2}\right|^{2}\left[\sqrt{E_{2}^{s}}-2 \Re\left\{\frac{\alpha_{1}}{\alpha_{2}}\right\} \rho \sqrt{E_{1}^{s}}\right] \mid \alpha_{1}, \alpha_{2}\right) \cdot Q\left(\sqrt{\frac{2 E_{1}^{s}\left(1-\rho^{2}\right)}{N_{0}}}\left|\alpha_{1}\right|\right) \\
& =Q\left(\frac{\left|\alpha_{2}\right|^{2} \sqrt{E_{2}^{s}}}{\sqrt{\frac{N_{0}}{2}\left|\alpha_{2}\right|^{2}}}\right) \cdot\left(1-Q\left(\sqrt{\frac{2 E_{1}^{s}\left(1-\rho^{2}\right)}{N_{0}}}\left|\alpha_{1}\right|\right)\right) \\
& +Q\left(\frac{\left|\alpha_{2}\right|^{2}\left[\sqrt{E_{2}^{s}}-2 \Re\left\{\alpha_{1} / \alpha_{2}\right\} \rho \sqrt{E_{1}^{s}}\right]}{\sqrt{\frac{N_{0}}{2}\left|\alpha_{2}\right|^{2}}}\right) \cdot Q\left(\sqrt{\frac{2 E_{1}^{s}\left(1-\rho^{2}\right)}{N_{0}}}\left|\alpha_{1}\right|\right) \\
& =Q\left(\sqrt{\frac{2 E_{2}^{s}}{N_{0}}}\left|\alpha_{2}\right|\right) \cdot\left(1-Q\left(\sqrt{\frac{2 E_{1}^{s}\left(1-\rho^{2}\right)}{N_{0}}}\left|\alpha_{1}\right|\right)\right) \\
& +Q\left(\frac{\left|\alpha_{2}\right|\left[\sqrt{2 E_{2}^{s}}-2 \Re\left\{\alpha_{1} / \alpha_{2}\right\} \rho \sqrt{2 E_{1}^{s}}\right]}{\sqrt{N_{0}}}\right) \cdot Q\left(\sqrt{\frac{2 E_{1}^{s}\left(1-\rho^{2}\right)}{N_{0}}}\left|\alpha_{1}\right|\right) \\
& =Q\left(\sqrt{\frac{2 E_{2}^{s}}{N_{0}}}\left|\alpha_{2}\right|\right) \cdot\left(1-Q\left(\sqrt{\frac{2 E_{1}^{s}\left(1-\rho^{2}\right)}{N_{0}}}\left|\alpha_{1}\right|\right)\right) \\
& +Q\left(\frac{\left|\alpha_{2}\right| \sqrt{2 E_{2}^{s}}-2 \Re\left\{\alpha_{1} \exp \left\{-j \operatorname{phase}\left(\alpha_{2}\right)\right\}\right\} \rho \sqrt{2 E_{1}^{s}}}{\sqrt{N_{0}}}\right) \cdot Q\left(\sqrt{\frac{2 E_{1}^{s}\left(1-\rho^{2}\right)}{N_{0}}}\left|\alpha_{1}\right|\right)
\end{aligned}
$$

where we have used (E.3) to evaluate $P\left(\hat{b}_{1}^{\mathrm{s}}=-1 \mid b_{1}^{\mathrm{s}}=1, \alpha_{1}, \alpha_{2}\right),(\mathrm{E} .5)$ to evaluate 


$$
\begin{aligned}
& P\left(\hat{b}_{2}^{\mathrm{s}}=1 \mid\left(b_{2}^{\mathrm{s}}=-1, b_{1}^{\mathrm{s}}=1, \hat{b}_{1}^{\mathrm{s}}=i\right), \alpha_{1}, \alpha_{2}\right), E\left[\left(\Re\left\{n_{2}^{\mathrm{s}}\right\}\right)^{2}\right]=E\left[\left|n_{2}^{\mathrm{s}}\right|^{2} / 2\right]=\left(N_{0} / 2\right)\left[\boldsymbol{R}_{\mathrm{s}}\right]_{22}= \\
& \left(N_{0} / 2\right)\left|\alpha_{2}\right|^{2} .
\end{aligned}
$$

Similarly, $P\left(\hat{b}_{2}^{\mathrm{s}}=1 \mid\left(b_{2}^{\mathrm{s}}=-1, b_{1}^{\mathrm{s}}=-1\right), \alpha_{1}, \alpha_{2}\right)$ can be obtained as:

$$
\begin{aligned}
& P\left(\hat{b}_{2}^{\mathrm{s}}=1 \mid\left(b_{2}^{\mathrm{s}}=-1, b_{1}^{\mathrm{s}}=-1\right), \alpha_{1}, \alpha_{2}\right) \\
& =P\left(\hat{b}_{2}^{\mathrm{s}}=1 \mid\left(b_{2}^{\mathrm{s}}=-1, b_{1}^{\mathrm{s}}=-1, \hat{b}_{1}^{\mathrm{s}}=1\right), \alpha_{1}, \alpha_{2}\right) \cdot P\left(\hat{b}_{1}^{\mathrm{s}}=1 \mid\left(b_{2}^{\mathrm{s}}=-1, b_{1}^{\mathrm{s}}=-1\right), \alpha_{1}, \alpha_{2}\right) \\
& +P\left(\hat{b}_{2}^{\mathrm{s}}=1 \mid\left(b_{2}^{\mathrm{s}}=-1, b_{1}^{\mathrm{s}}=-1, \hat{b}_{1}^{\mathrm{s}}=-1\right), \alpha_{1}, \alpha_{2}\right) \cdot P\left(\hat{b}_{1}^{\mathrm{s}}=-1 \mid\left(b_{2}^{\mathrm{s}}=-1, b_{1}^{\mathrm{s}}=-1\right), \alpha_{1}, \alpha_{2}\right) \\
& =P\left(\hat{b}_{2}^{\mathrm{s}}=1 \mid\left(b_{2}^{\mathrm{s}}=-1, b_{1}^{\mathrm{s}}=-1, \hat{b}_{1}^{\mathrm{s}}=1\right), \alpha_{1}, \alpha_{2}\right) \cdot P\left(\hat{b}_{1}^{\mathrm{s}}=1 \mid b_{1}^{\mathrm{s}}=-1, \alpha_{1}, \alpha_{2}\right) \\
& +P\left(\hat{b}_{2}^{\mathrm{s}}=1 \mid\left(b_{2}^{\mathrm{s}}=-1, b_{1}^{\mathrm{s}}=-1, \hat{b}_{1}^{\mathrm{s}}=-1\right), \alpha_{1}, \alpha_{2}\right) \cdot P\left(\hat{b}_{1}^{\mathrm{s}}=-1 \mid b_{1}^{\mathrm{s}}=-1, \alpha_{1}, \alpha_{2}\right) \\
& =P\left(\hat{b}_{2}^{\mathrm{s}}=1 \mid\left(b_{2}^{\mathrm{s}}=-1, b_{1}^{\mathrm{s}}=-1, \hat{b}_{1}^{\mathrm{s}}=1\right), \alpha_{1}, \alpha_{2}\right) \cdot P\left(\hat{b}_{1}^{\mathrm{s}}=1 \mid b_{1}^{\mathrm{s}}=-1, \alpha_{1}, \alpha_{2}\right) \\
& +P\left(\hat{b}_{2}^{\mathrm{s}}=1 \mid\left(b_{2}^{\mathrm{s}}=-1, b_{1}^{\mathrm{s}}=-1, \hat{b}_{1}^{\mathrm{s}}=-1\right), \alpha_{1}, \alpha_{2}\right) \cdot\left(1-P\left(\hat{b}_{1}^{\mathrm{s}}=1 \mid b_{1}^{\mathrm{s}}=-1, \alpha_{1}, \alpha_{2}\right)\right) \\
& =P\left(-1+\frac{1}{\left|\alpha_{2}\right|^{2} \sqrt{E_{2}^{s}}} \Re\left\{n_{2}^{\mathrm{s}}\right\}+\Re\left\{\frac{\alpha_{1}}{\alpha_{2}}\right\} \rho \sqrt{\frac{E_{1}^{s}}{E_{2}^{s}}}(-1-1)>0 \mid \alpha_{1}, \alpha_{2}\right) \\
& Q\left(\sqrt{\frac{2 E_{1}^{s}\left(1-\rho^{2}\right)}{N_{0}}}\left|\alpha_{1}\right|\right) \\
& +P\left(-1+\frac{1}{\left|\alpha_{2}\right|^{2} \sqrt{E_{2}^{s}}} \Re\left\{n_{2}^{\mathrm{s}}\right\}+\Re\left\{\frac{\alpha_{1}}{\alpha_{2}}\right\} \rho \sqrt{\frac{E_{1}^{s}}{E_{2}^{s}}}(-1-(-1))>0 \mid \alpha_{1}, \alpha_{2}\right) \\
& \cdot\left(1-Q\left(\sqrt{\frac{2 E_{1}^{s}\left(1-\rho^{2}\right)}{N_{0}}}\left|\alpha_{1}\right|\right)\right) \\
& =P\left(\Re\left\{n_{2}^{\mathrm{s}}\right\}>\left|\alpha_{2}\right|^{2}\left[\sqrt{E_{2}^{s}}+2 \Re\left\{\frac{\alpha_{1}}{\alpha_{2}}\right\} \rho \sqrt{E_{1}^{s}}\right] \mid \alpha_{1}, \alpha_{2}\right) \cdot Q\left(\sqrt{\frac{2 E_{1}^{s}\left(1-\rho^{2}\right)}{N_{0}}}\left|\alpha_{1}\right|\right) \\
& +P\left(\Re\left\{n_{2}^{\mathrm{s}}\right\}>\left|\alpha_{2}\right|^{2} \sqrt{E_{2}^{s}} \mid \alpha_{1}, \alpha_{2}\right) \cdot\left(1-Q\left(\sqrt{\frac{2 E_{1}^{s}\left(1-\rho^{2}\right)}{N_{0}}}\left|\alpha_{1}\right|\right)\right)
\end{aligned}
$$




$$
\begin{aligned}
= & Q\left(\frac{\left|\alpha_{2}\right|^{2}\left[\sqrt{E_{2}^{s}}+2 \Re\left\{\alpha_{1} / \alpha_{2}\right\} \rho \sqrt{E_{1}^{s}}\right]}{\sqrt{\frac{N_{0}}{2}\left|\alpha_{2}\right|^{2}}}\right) \cdot Q\left(\sqrt{\frac{2 E_{1}^{s}\left(1-\rho^{2}\right)}{N_{0}}}\left|\alpha_{1}\right|\right) \\
& +Q\left(\frac{\left|\alpha_{2}\right|^{2} \sqrt{E_{2}^{s}}}{\sqrt{\frac{N_{0}}{2}\left|\alpha_{2}\right|^{2}}}\right) \cdot\left(1-Q\left(\sqrt{\frac{2 E_{1}^{s}\left(1-\rho^{2}\right)}{N_{0}}}\left|\alpha_{1}\right|\right)\right) \\
= & Q\left(\frac{\left.\left|\alpha_{2}\right| \sqrt{2 E_{2}^{s}}+2 \Re\left\{\alpha_{1} \exp \left\{-j \operatorname{phase}\left(\alpha_{2}\right)\right\}\right\} \rho \sqrt{2 E_{1}^{s}}\right) \cdot Q\left(\sqrt{\frac{2 E_{1}^{s}\left(1-\rho^{2}\right)}{N_{0}}}\left|\alpha_{1}\right|\right)}{\sqrt{N_{0}}}\right) \\
& +Q\left(\sqrt{\frac{2 E_{2}^{s}}{N_{0}}}\left|\alpha_{2}\right|\right) \cdot\left(1-Q\left(\sqrt{\frac{2 E_{1}^{s}\left(1-\rho^{2}\right)}{N_{0}}}\left|\alpha_{1}\right|\right)\right)
\end{aligned}
$$

Hence substituting (E.7) and (E.8) into (E.6) yields

$$
\begin{aligned}
& P_{e, 2 \mid \alpha_{1}, \alpha_{2}}\left(N_{0}\right)=\frac{1}{2} Q\left(\sqrt{\frac{2 E_{2}^{s}}{N_{0}}}\left|\alpha_{2}\right|\right) \cdot\left(1-Q\left(\sqrt{\frac{2 E_{1}^{s}\left(1-\rho^{2}\right)}{N_{0}}}\left|\alpha_{1}\right|\right)\right) \\
& +\frac{1}{2} Q\left(\frac{\left|\alpha_{2}\right| \sqrt{2 E_{2}^{s}}-2 \Re\left\{\alpha_{1} \exp \left\{-j \operatorname{phase}\left(\alpha_{2}\right)\right\}\right\} \rho \sqrt{2 E_{1}^{s}}}{\sqrt{N_{0}}}\right) \cdot Q\left(\sqrt{\frac{2 E_{1}^{s}\left(1-\rho^{2}\right)}{N_{0}}}\left|\alpha_{1}\right|\right) \\
& +\frac{1}{2} Q\left(\frac{\left|\alpha_{2}\right| \sqrt{2 E_{2}^{s}}+2 \Re\left\{\alpha_{1} \exp \left\{-j \text { phase }\left(\alpha_{2}\right)\right\}\right\} \rho \sqrt{2 E_{1}^{s}}}{\sqrt{N_{0}}}\right) \cdot Q\left(\sqrt{\frac{2 E_{1}^{s}\left(1-\rho^{2}\right)}{N_{0}}}\left|\alpha_{1}\right|\right) \\
& +\frac{1}{2} Q\left(\sqrt{\frac{2 E_{2}^{s}}{N_{0}}}\left|\alpha_{2}\right|\right) \cdot\left(1-Q\left(\sqrt{\frac{2 E_{1}^{s}\left(1-\rho^{2}\right)}{N_{0}}}\left|\alpha_{1}\right|\right)\right) \\
& =Q\left(\sqrt{\frac{2 E_{2}^{s}}{N_{0}}}\left|\alpha_{2}\right|\right) \cdot\left(1-Q\left(\sqrt{\frac{2 E_{1}^{s}\left(1-\rho^{2}\right)}{N_{0}}}\left|\alpha_{1}\right|\right)\right) \\
& +\frac{1}{2} Q\left(\sqrt{\frac{2 E_{1}^{s}\left(1-\rho^{2}\right)}{N_{0}}}\left|\alpha_{1}\right|\right)\left[Q\left(\frac{\left|\alpha_{2}\right| \sqrt{2 E_{2}^{s}}-2 \Re\left\{\alpha_{1} \exp \left\{-j \operatorname{phase}\left(\alpha_{2}\right)\right\}\right\} \rho \sqrt{2 E_{1}^{s}}}{\sqrt{N_{0}}}\right)\right. \\
& \left.+Q\left(\frac{\left|\alpha_{2}\right| \sqrt{2 E_{2}^{s}}+2 \Re\left\{\alpha_{1} \exp \left\{-j \operatorname{phase}\left(\alpha_{2}\right)\right\}\right\} \rho \sqrt{2 E_{1}^{s}}}{\sqrt{N_{0}}}\right)\right]
\end{aligned}
$$


Using (E.2)

$$
\begin{aligned}
& P_{e, 2 \mid \alpha_{1}, \operatorname{phase}\left(\alpha_{2}\right)}\left(N_{0}\right)=E\left[P_{e, 2 \mid \alpha_{1}, \alpha_{2}}\left(N_{0}\right)\right]=\int_{0}^{+\infty} P_{R}(r) P_{e, 2 \mid \alpha_{1}, \alpha_{2}}\left(N_{0}\right) d r \quad r=\left|\alpha_{2}\right| \\
& =\int_{0}^{+\infty} r e^{-r^{2} / 2} P_{e, 2 \mid \alpha_{1}, \alpha_{2}}\left(N_{0}\right) d r \\
& =\int_{0}^{+\infty} r e^{-r^{2} / 2} Q\left(\sqrt{\frac{2 E_{2}^{s}}{N_{0}}} r\right) \cdot\left(1-Q\left(\sqrt{\frac{2 E_{1}^{s}\left(1-\rho^{2}\right)}{N_{0}}}\left|\alpha_{1}\right|\right)\right) d r \\
& +\frac{1}{2} \int_{0}^{+\infty} r e^{-r^{2} / 2} Q\left(\sqrt{\frac{2 E_{1}^{s}\left(1-\rho^{2}\right)}{N_{0}}}\left|\alpha_{1}\right|\right) Q\left(\frac{r \sqrt{2 E_{2}^{s}}-2 \Re\left\{\alpha_{1} e^{-j \operatorname{phase}\left(\alpha_{2}\right)}\right\} \rho \sqrt{2 E_{1}^{s}}}{\sqrt{N_{0}}}\right) d r \\
& +\frac{1}{2} \int_{0}^{+\infty} r e^{-r^{2} / 2} Q\left(\sqrt{\frac{2 E_{1}^{s}\left(1-\rho^{2}\right)}{N_{0}}}\left|\alpha_{1}\right|\right) Q\left(\frac{r \sqrt{2 E_{2}^{s}}+2 \Re\left\{\alpha_{1} e^{-j \text { phase }\left(\alpha_{2}\right)}\right\} \rho \sqrt{2 E_{1}^{s}}}{\sqrt{N_{0}}}\right) d r \\
& =\left(1-Q\left(\sqrt{\frac{2 E_{1}^{s}\left(1-\rho^{2}\right)}{N_{0}}}\left|\alpha_{1}\right|\right)\right) \int_{0}^{+\infty} r e^{-r^{2} / 2} Q\left(\sqrt{\frac{2 E_{2}^{s}}{N_{0}}} r\right) d r \\
& +\frac{1}{2} Q\left(\sqrt{\frac{2 E_{1}^{s}\left(1-\rho^{2}\right)}{N_{0}}}\left|\alpha_{1}\right|\right) \int_{0}^{+\infty} r e^{-r^{2} / 2} Q\left(\frac{r \sqrt{2 E_{2}^{s}}-2 \Re\left\{\alpha_{1} e^{-j \text { phase }\left(\alpha_{2}\right)}\right\} \rho \sqrt{2 E_{1}^{s}}}{\sqrt{N_{0}}}\right) d r \\
& +\frac{1}{2} Q\left(\sqrt{\frac{2 E_{1}^{s}\left(1-\rho^{2}\right)}{N_{0}}}\left|\alpha_{1}\right|\right) \int_{0}^{+\infty} r e^{-r^{2} / 2} Q\left(\frac{r \sqrt{2 E_{2}^{s}}+2 \Re\left\{\alpha_{1} e^{-j \operatorname{phase}\left(\alpha_{2}\right)}\right\} \rho \sqrt{2 E_{1}^{s}}}{\sqrt{N_{0}}}\right) d r \\
& =\left(1-Q\left(\sqrt{\frac{2 E_{1}^{s}\left(1-\rho^{2}\right)}{N_{0}}}\left|\alpha_{1}\right|\right)\right) \frac{1}{2}\left[1-\frac{\sqrt{\frac{2 E_{2}^{s}}{N_{0}}}}{\sqrt{1+\frac{2 E_{2}^{s}}{N_{0}}}}\right] \\
& +\frac{1}{2} Q\left(\sqrt{\frac{2 E_{1}^{s}\left(1-\rho^{2}\right)}{N_{0}}}\left|\alpha_{1}\right|\right)\left[1-\frac{\frac{\sqrt{2 E_{2}^{s}}}{\sqrt{N_{0}}}}{\sqrt{1+\frac{2 E_{2}^{s}}{N_{0}}}} \exp \left\{-\frac{\frac{\left[-2 \Re\left\{\alpha_{1} \exp \left\{-j \operatorname{phase}\left(\alpha_{2}\right)\right\}\right\} \rho \sqrt{2 E_{1}^{s}}\right]^{2}}{N_{0}}}{2\left(1+\frac{2 E_{2}^{s}}{N_{0}}\right)}\right\}\right]
\end{aligned}
$$




$$
\begin{aligned}
&=\left(1-Q\left(\sqrt{\frac{2 E_{1}^{s}\left(1-\rho^{2}\right)}{N_{0}}}\left|\alpha_{1}\right|\right)\right) \frac{1}{2}\left[1-\frac{1}{\sqrt{1+\frac{N_{0}}{2 E_{2}^{s}}}}\right] \\
&+\frac{1}{2} Q\left(\sqrt{\frac{2 E_{1}^{s}\left(1-\rho^{2}\right)}{N_{0}}}\left|\alpha_{1}\right|\right) \\
& \cdot\left[1-\frac{1}{\sqrt{1+\frac{N_{0}}{2 E_{2}^{s}}}} \exp \left\{-\frac{4\left|\alpha_{1}\right|^{2} \rho^{2} 2 E_{1}^{s}\left[\Re\left\{e^{-j \text { phase }\left(\alpha_{2}\right)} e^{j \text { phase }\left(\alpha_{1}\right)}\right\}\right]^{2}}{2 N_{0}\left(1+\frac{2 E_{2}^{s}}{N_{0}}\right)}\right\}\right]
\end{aligned}
$$

where we have used

$$
\begin{aligned}
& \int_{0}^{+\infty} r e^{-r^{2} / 2} Q(\alpha r+\beta) d r+\int_{0}^{+\infty} r e^{-r^{2} / 2} Q(\alpha r-\beta) d r \\
& =Q(\beta)-\frac{\alpha}{\sqrt{1+\alpha^{2}}} \frac{1}{2} \exp \left\{-\frac{\beta^{2}}{2\left(1+\alpha^{2}\right)}\right\}+Q(-\beta)-\frac{\alpha}{\sqrt{1+\alpha^{2}}} \frac{1}{2} \exp \left\{-\frac{\beta^{2}}{2\left(1+\alpha^{2}\right)}\right\} \\
& =Q(\beta)+Q(-\beta)-\frac{\alpha}{\sqrt{1+\alpha^{2}}} \exp \left\{-\frac{\beta^{2}}{2\left(1+\alpha^{2}\right)}\right\} \\
& =1-\frac{\alpha}{\sqrt{1+\alpha^{2}}} \exp \left\{-\frac{\beta^{2}}{2\left(1+\alpha^{2}\right)}\right\} \quad \alpha \geq 0
\end{aligned}
$$

since

$$
\begin{aligned}
\int_{0}^{+\infty} r e^{-r^{2} / 2} Q(\alpha r+\beta) d r=\left[-e^{-r^{2} / 2} Q(\alpha r+\beta)\right]_{0}^{\infty}-\int_{0}^{+\infty}-e^{-r^{2} / 2}(-1) \frac{\alpha}{\sqrt{2 \pi}} e^{-(\alpha r+\beta)^{2} / 2} d r \\
\quad=Q(\beta)-\frac{\alpha}{\sqrt{2 \pi}} \int_{0}^{+\infty} e^{-\left[\left(1+\alpha^{2}\right) r^{2}+2 \alpha \beta r+\beta^{2}\right] / 2} d r \\
\quad=Q(\beta)-\frac{\alpha}{\sqrt{2 \pi}} \int_{0}^{+\infty} e^{-\left(1+\alpha^{2}\right)\left[\left(r+\frac{\alpha \beta}{1+\alpha^{2}}\right)^{2}+\frac{\beta^{2}}{1+\alpha^{2}}-\frac{\alpha^{2} \beta^{2}}{\left(1+\alpha^{2}\right)^{2}}\right] / 2} d r \\
=Q(\beta)-\alpha \sqrt{\frac{1}{1+\alpha^{2}}} \exp \left\{-\frac{\beta^{2}}{2\left(1+\alpha^{2}\right)}\right\} \frac{1}{\sqrt{2 \pi \frac{1}{1+\alpha^{2}}}} \int_{0}^{+\infty} e^{-\frac{\left(r+\frac{\alpha \beta}{1+\alpha^{2}}\right)^{2}}{\frac{1}{1+\alpha^{2}}}} d r \\
=Q(\beta)-\frac{\alpha}{\sqrt{1+\alpha^{2}}} \frac{1}{2} \exp \left\{-\frac{\beta^{2}}{2\left(1+\alpha^{2}\right)}\right\}
\end{aligned}
$$




$$
\begin{aligned}
& P_{e, 2 \mid \operatorname{phase}\left(\alpha_{1}\right), \operatorname{phase}\left(\alpha_{2}\right)}\left(N_{0}\right)=E\left[P_{e, 2 \mid \alpha_{1}, \operatorname{phase}\left(\alpha_{2}\right)}\left(N_{0}\right)\right]=\int_{0}^{+\infty} P_{R}(r) P_{e, 2 \mid \alpha_{1}, \operatorname{phase}\left(\alpha_{2}\right)}\left(N_{0}\right) d r \\
& =\int_{0}^{+\infty} r e^{-r^{2} / 2} P_{e, 2 \mid \alpha_{1}, \operatorname{phase}\left(\alpha_{2}\right)}\left(N_{0}\right) d r \quad r=\left|\alpha_{1}\right| \\
& =\int_{0}^{+\infty} r e^{-r^{2} / 2}\left(1-Q\left(\sqrt{\frac{2 E_{1}^{s}\left(1-\rho^{2}\right)}{N_{0}}} r\right)\right) \frac{1}{2}\left[1-\frac{1}{\sqrt{1+\frac{N_{0}}{2 E_{2}^{s}}}}\right] d r \\
& +\int_{0}^{+\infty} r e^{-r^{2} / 2} \frac{1}{2} Q\left(\sqrt{\frac{2 E_{1}^{s}\left(1-\rho^{2}\right)}{N_{0}} r}\right) d r \\
& -\int_{0}^{+\infty} r e^{-r^{2} / 2} \frac{1}{2} Q\left(\sqrt{\frac{2 E_{1}^{s}\left(1-\rho^{2}\right)}{N_{0}}} r\right) \frac{\exp \left\{-\frac{4 r^{2} \rho^{2} 2 E_{1}^{s}\left[\Re\left\{e^{-j \text { phase }\left(\alpha_{2}\right)} e^{j \text { phase }\left(\alpha_{1}\right)}\right\}\right]^{2}}{2 N_{0}\left(1+\frac{2 E_{2}^{2}}{N_{0}}\right)}\right\}}{\sqrt{1+\frac{N_{0}}{2 E_{2}^{s}}}} d r \\
& =\frac{1}{2}\left[1-\frac{1}{\sqrt{1+\frac{N_{0}}{2 E_{2}^{s}}}}\right]\left[\left[-e^{-r^{2} / 2}\right]_{0}^{\infty}-\frac{1}{2}\left[1-\frac{\sqrt{\frac{2 E_{1}^{s}\left(1-\rho^{2}\right)}{N_{0}}}}{\sqrt{1+\frac{2 E_{1}^{s}\left(1-\rho^{2}\right)}{N_{0}}}}\right]\right] \\
& +\frac{1}{2} \frac{1}{2}\left[1-\frac{\sqrt{\frac{2 E_{1}^{s}\left(1-\rho^{2}\right)}{N_{0}}}}{\sqrt{1+\frac{2 E_{1}^{s}\left(1-\rho^{2}\right)}{N_{0}}}}\right]
\end{aligned}
$$

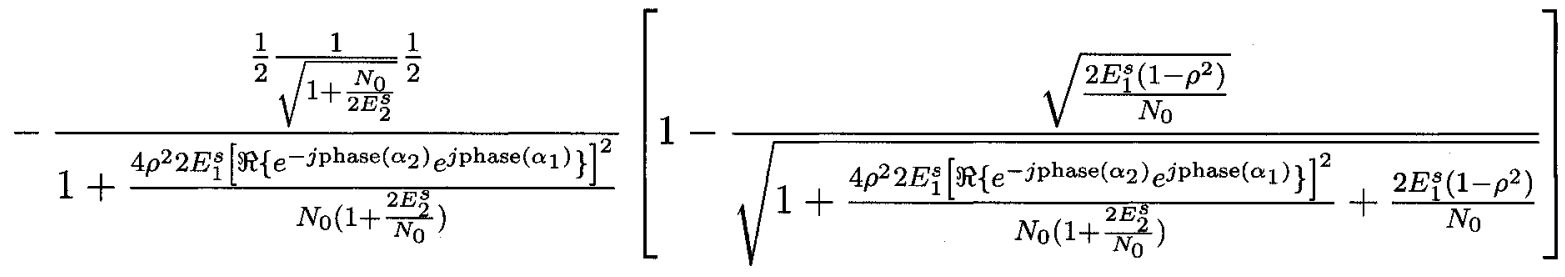

$$
\begin{aligned}
& =\frac{1}{2} \frac{1}{2}\left[1-\frac{1}{\sqrt{1+\frac{N_{0}}{2 E_{2}^{s}}}}\right]\left[1+\frac{1}{\sqrt{1+\frac{N_{0}}{2 E_{1}^{s}\left(1-\rho^{2}\right)}}}\right]+\frac{1}{2} \frac{1}{2}\left[1-\frac{1}{\sqrt{1+\frac{N_{0}}{2 E_{1}^{s}\left(1-\rho^{2}\right)}}}\right] \\
& -\frac{\frac{1}{2} \frac{1}{\sqrt{1+\frac{N_{0}}{2 E_{2}^{s}}}} \frac{1}{2}}{1+\frac{4 \rho^{2} 2 E_{1}^{s}\left[\Re \left\{e^{\left.\left.-j \text { phase }\left(\alpha_{2}\right) e^{j \text { phase }\left(\alpha_{1}\right)}\right\}\right]^{2}}\right.\right.}{N_{0}\left(1+\frac{2 E_{2}^{s}}{N_{0}}\right)}}\left[1-\frac{\sqrt{\frac{2 E_{1}^{s}\left(1-\rho^{2}\right)}{N_{0}}}}{\sqrt{1+\frac{4 \rho^{2} 2 E_{1}^{s}\left[\Re\left\{e^{-j \text { phase }\left(\alpha_{2}\right)} e^{j \text { phase }\left(\alpha_{1}\right)}\right\}\right]^{2}}{N_{0}\left(1+\frac{2 E_{2}^{s}}{N_{0}}\right)}+\frac{2 E_{1}^{s}\left(1-\rho^{2}\right)}{N_{0}}}}\right]
\end{aligned}
$$


where we have used

$$
\begin{aligned}
& \int_{0}^{+\infty} r e^{-r^{2} / 2} Q(\alpha r) e^{-r^{2} \beta / 2} d r=\int_{0}^{+\infty} r e^{-(1+\beta) r^{2} / 2} Q(\alpha r) d r \\
& =\frac{1}{1+\beta} \int_{0}^{+\infty} u e^{-u^{2} / 2} Q\left(\frac{\alpha}{\sqrt{1+\beta}} u\right) d u=\frac{1}{1+\beta} \frac{1}{2}\left[1-\frac{\frac{\alpha}{\sqrt{1+\beta}}}{\sqrt{1+\frac{\alpha^{2}}{1+\beta}}}\right] \\
& =\frac{1}{1+\beta} \frac{1}{2}\left[1-\frac{\alpha}{\sqrt{1+\beta+\alpha^{2}}}\right] \quad \alpha \geq 0
\end{aligned}
$$

$$
\begin{aligned}
& P_{e, 2}\left(N_{0}\right)=E\left[P_{e, 2 \mid \operatorname{phase}\left(\alpha_{1}\right), \operatorname{phase}\left(\alpha_{2}\right)}\left(N_{0}\right)\right] \\
& =\int_{-\pi}^{\pi} \int_{-\pi}^{\pi} P_{e, 2 \mid \operatorname{phase}\left(\alpha_{1}\right), \operatorname{phase}\left(\alpha_{2}\right)}\left(N_{0}\right) \frac{1}{2 \pi} \frac{1}{2 \pi} d \theta_{1} d \theta_{2} \quad \theta_{1}=\operatorname{phase}\left(\alpha_{1}\right) \quad \theta_{2}=\operatorname{phase}\left(\alpha_{2}\right) \\
& =\left(\frac{1}{2 \pi}\right)^{2} \int_{-\pi}^{\pi} \int_{-\pi}^{\pi} \frac{1}{4}\left[1-\frac{1}{\sqrt{1+\frac{N_{0}}{2 E_{2}^{s}}}}\right]\left[1+\frac{1}{\sqrt{1+\frac{N_{0}}{2 E_{1}^{s}\left(1-\rho^{2}\right)}}}\right] d \theta_{1} d \theta_{2} \\
& +\left(\frac{1}{2 \pi}\right)^{2} \int_{-\pi}^{\pi} \int_{-\pi}^{\pi} \frac{1}{4}\left[1-\frac{1}{\sqrt{1+\frac{N_{0}}{2 E_{1}^{s}\left(1-\rho^{2}\right)}}}\right] d \theta_{1} d \theta_{2} \\
& +\left(\frac{1}{2 \pi}\right)^{2} \int_{-\pi}^{\pi} \int_{-\pi}^{\pi}(-1) \frac{1}{4} \frac{1}{\sqrt{1+\frac{N_{0}}{2 E_{2}^{s}}}} \frac{1}{1+\frac{4 \rho^{2} 2 E_{1}^{s} \cos ^{2}\left(\theta_{1}-\theta_{2}\right)}{N_{0}\left(1+\frac{2 E_{2}^{s}}{N_{0}}\right)}} d \theta_{1} d \theta_{2} \\
& +\left(\frac{1}{2 \pi}\right)^{2} \int_{-\pi}^{\pi} \int_{-\pi}^{\pi} \frac{1}{4} \frac{1}{\sqrt{1+\frac{N_{0}}{2 E_{2}^{s}}}} \frac{1}{1+\frac{4 \rho^{2} 2 E_{1}^{s} \cos ^{2}\left(\theta_{1}-\theta_{2}\right)}{N_{0}\left(1+\frac{2 E_{2}^{s}}{N_{0}}\right)}} \frac{\sqrt{\frac{2 E_{1}^{s}\left(1-\rho^{2}\right)}{N_{0}}}}{\sqrt{1+\frac{4 \rho^{2} 2 E_{1}^{s} \cos ^{2}\left(\theta_{1}-\theta_{2}\right)}{N_{0}\left(1+\frac{2 E_{2}^{s}}{N_{0}}\right)}+\frac{2 E_{1}^{s}\left(1-\rho^{2}\right)}{N_{0}}}} d \theta_{1} d \theta_{2} \\
& =\frac{1}{4}\left[1-\frac{1}{\sqrt{1+\frac{N_{0}}{2 E_{2}^{s}}}}\right]\left[1+\frac{1}{\sqrt{1+\frac{N_{0}}{2 E_{1}^{s}\left(1-\rho^{2}\right)}}}\right]+\frac{1}{4}\left[1-\frac{1}{\sqrt{1+\frac{N_{0}}{2 E_{1}^{s}\left(1-\rho^{2}\right)}}}\right] \\
& -\frac{1}{4}\left(\frac{1}{2 \pi}\right)^{2} \frac{1}{\sqrt{1+\frac{N_{0}}{2 E_{2}^{s}}}} \int_{-\pi}^{\pi} \int_{-\pi-\theta_{2}}^{\pi-\theta_{2}} \frac{1}{1+\frac{4 \rho^{2} 2 E_{1}^{s} \cos ^{2}(u)}{N_{0}\left(1+\frac{2 E_{2}^{s}}{N_{0}}\right)}} d u d \theta_{2} \quad u=\theta_{1}-\theta_{2} \\
& +\frac{1}{4}\left(\frac{1}{2 \pi}\right)^{2} \frac{1}{\sqrt{1+\frac{N_{0}}{2 E_{2}^{s}}}} \int_{-\pi}^{\pi} \int_{-\pi-\theta_{2}}^{\pi-\theta_{2}} \frac{1}{1+\frac{4 \rho^{2} 2 E_{1}^{s} \cos ^{2}(u)}{N_{0}\left(1+\frac{2 E_{2}^{s}}{N_{0}}\right)}} \frac{\sqrt{\frac{2 E_{1}^{s}\left(1-\rho^{2}\right)}{N_{0}}}}{\sqrt{1+\frac{4 \rho^{2} 2 E_{1}^{s} \cos ^{2}(u)}{N_{0}\left(1+\frac{2 E_{2}^{s}}{N_{0}}\right)}+\frac{2 E_{1}^{s}\left(1-\rho^{2}\right)}{N_{0}}}} d u d \theta_{2}
\end{aligned}
$$




$$
\begin{aligned}
= & \frac{1}{4}\left[1-\frac{1}{\sqrt{1+\frac{N_{0}}{2 E_{2}^{s}}}}\right]\left[1+\frac{1}{\sqrt{1+\frac{N_{0}}{2 E_{1}^{s}\left(1-\rho^{2}\right)}}}\right]+\frac{1}{4}\left[1-\frac{1}{\sqrt{1+\frac{N_{0}}{2 E_{1}^{s}\left(1-\rho^{2}\right)}}}\right] \\
& -\frac{1}{4}\left(\frac{1}{2 \pi}\right)^{2} \frac{1}{\sqrt{1+\frac{N_{0}}{2 E_{2}^{s}}}} \int_{-\pi}^{\pi} \int_{-\pi}^{\pi} \frac{1}{1+\alpha \cos ^{2}(u)} d u d \theta_{2} \\
& +\frac{1}{4}\left(\frac{1}{2 \pi}\right)^{2} \frac{1}{\sqrt{1+\frac{N_{0}}{2 E_{2}^{s}}}} \int_{-\pi}^{\pi} \int_{-\pi}^{\pi} \frac{1}{1+\alpha \cos ^{2}(u)} \frac{\beta}{\sqrt{1+\alpha \cos ^{2}(u)+\beta^{2}}} d u d \theta_{2}
\end{aligned}
$$

where we have used that $\cos ^{2}(u)$ is periodic and the integration is over one period, and $\alpha, \beta$ are defined as

$$
\alpha=\frac{4 \rho^{2} 2 E_{1}^{s}}{N_{0}\left(1+\frac{2 E_{2}^{s}}{N_{0}}\right)} \quad \beta=\sqrt{\frac{2 E_{1}^{s}\left(1-\rho^{2}\right)}{N_{0}}}
$$

$$
\begin{aligned}
& \int_{-\pi}^{\pi} \frac{1}{1+\alpha \cos ^{2}(u)} d u=2 \int_{0}^{\pi} \frac{1}{1+\alpha \cos ^{2}(u)} d u=2 \int_{-1}^{1} \frac{1}{1+\alpha v^{2}} \frac{d v}{\sqrt{1-v^{2}}} \quad v=\cos (u) \\
& =2\left[\frac{1}{\sqrt{d(a d-b c)}} \operatorname{tg}^{-1}\left[\frac{v \sqrt{a d-b c}}{\sqrt{b\left(c v^{2}+d\right)}}\right]\right]_{-1}^{1} \quad(a=\alpha b=1 c=-1 d=1) \\
& =2\left[\frac{1}{\sqrt{1+\alpha}} \operatorname{tg}^{-1}\left[\frac{v \sqrt{1+\alpha}}{\sqrt{1-v^{2}}}\right]\right]_{-1}^{1}=\frac{2}{\sqrt{1+\alpha}}\left\{\operatorname{tg}^{-1}\left[\frac{(1) \sqrt{1+\alpha}}{\sqrt{0}}\right]-\operatorname{tg}^{-1}\left[\frac{(-1) \sqrt{1+\alpha}}{\sqrt{0}}\right]\right\} \\
& =\frac{2}{\sqrt{1+\alpha}} \pi
\end{aligned}
$$

where we have used eq. 3.3 .49 of [134]

$$
\int \frac{d x}{\left(a x^{2}+b\right) \sqrt{c x^{2}+d}}= \begin{cases}\frac{1}{\sqrt{d(a d-b c)}} \operatorname{tg}^{-1}\left[\frac{x \sqrt{a d-b c}}{\sqrt{b\left(c x^{2}+d\right)}}\right] & a d>b c \\ \frac{1}{2 \sqrt{b(b c-a d)}} \ln \left|\frac{\sqrt{b\left(c x^{2}+d\right)}+x \sqrt{b c-a d}}{\sqrt{b\left(c x^{2}+d\right)}-x \sqrt{b c-a d}}\right| & b c>a d\end{cases}
$$




$$
\begin{aligned}
& \int_{-\pi}^{\pi} \frac{1}{1+\alpha \cos ^{2}(u)} \frac{\beta}{\sqrt{1+\alpha \cos ^{2}(u)+\beta^{2}}} d u=2 \int_{0}^{\pi} \frac{1}{1+\alpha \cos ^{2}(u)} \frac{\beta}{\sqrt{1+\alpha \cos ^{2}(u)+\beta^{2}}} d u \\
&=2 \int_{0}^{\pi} \frac{1}{1+\alpha\left(1-\sin ^{2}(u)\right)} \frac{\beta}{\sqrt{1+\beta^{2}+\alpha\left(1-\sin ^{2}(u)\right)}} d u \\
&=2 \int_{0}^{\pi} \frac{1}{1+\alpha-\alpha \sin ^{2}(u)} \frac{\beta}{\sqrt{1+\beta^{2}+\alpha-\alpha \sin ^{2}(u)}} d u \\
&=2 \frac{\beta}{(1+\alpha) \sqrt{1+\beta^{2}+\alpha}} \int_{0}^{\pi} \frac{1}{1-\frac{\alpha}{1+\alpha} \sin ^{2}(u)} \frac{1}{\sqrt{1-\frac{\alpha}{1+\beta^{2}+\alpha} \sin ^{2}(u)}} d u \\
&=2 \frac{\beta}{(1+\alpha) \sqrt{1+\beta^{2}+\alpha}}\left[\int_{0}^{\frac{\pi}{2}} \frac{1}{1-\frac{\alpha}{1+\alpha} \sin ^{2}(u)} \frac{1}{\sqrt{1-\frac{\alpha}{1+\beta^{2}+\alpha} \sin ^{2}(u)}} d u\right. \\
&\left.\quad+\int_{\frac{\pi}{2}}^{\pi} \frac{1}{1-\frac{\alpha}{1+\alpha} \sin ^{2}(u)} \frac{1}{\sqrt{1-\frac{\alpha}{1+\beta^{2}+\alpha} \sin ^{2}(u)}} d u\right] \\
&= 2 \frac{\beta}{(1+\alpha) \sqrt{1+\beta^{2}+\alpha}}\left[\int_{0}^{\frac{\pi}{2}} \frac{1}{1-\frac{\alpha}{1+\alpha} \sin ^{2}(u)} \frac{1}{\sqrt{1-\frac{\alpha}{1+\beta^{2}+\alpha} \sin ^{2}(u)}} d u\right. \\
&+\int_{\frac{\pi}{2}}^{0} \frac{1}{1-\frac{\alpha}{1+\alpha} \sin ^{2}(\pi-v)} \frac{1}{\left.\sqrt{1-\frac{\alpha}{1+\beta^{2}+\alpha} \sin ^{2}(\pi-v)}(-1) d v\right]} v=\pi-u \\
&= 4 \frac{\beta}{(1+\alpha) \sqrt{1+\beta^{2}+\alpha}} \int_{0}^{\frac{\pi}{2}} \frac{1}{1-\frac{\alpha}{1+\alpha} \sin ^{2}(u)} \frac{1}{\sqrt{1-\frac{\alpha}{1+\beta^{2}+\alpha} \sin ^{2}(u)}} d u \\
&= \frac{\beta}{(1+\alpha) \sqrt{1+\beta^{2}+\alpha}} \prod\left(\frac{\pi}{2}, \frac{\alpha}{1+\alpha}, \sqrt{\left.\frac{\alpha}{1+\alpha+\beta^{2}}\right)}\right.
\end{aligned}
$$

where $\prod(\phi, n, k)$ is the elliptic integral of the third kind $[135,134]^{1}$ defined as

$$
\prod(\phi, n, k)=\int_{0}^{\phi} \frac{d u}{\left(1-n \sin ^{2} u\right) \sqrt{1-k^{2} \sin ^{2} u}} d u=\int_{0}^{\sin \phi} \frac{d x}{\left(1-n x^{2}\right) \sqrt{\left(1-x^{2}\right)\left(1-k^{2} x^{2}\right)}}
$$

where $k=\sin \alpha(|k|<1)$ is the modulus of the integral, $\alpha$ is the modular angle, and $\prod\left(\frac{\pi}{2}, n, k\right)$ is the complete elliptic integral of the third kind. Substituting (E.12) and

\footnotetext{
${ }^{1}$ Note that there is an alternate definition of the elliptic integral of the third kind where a plus sign in front of $n$ is used, however this derivation yields to the elliptic integral of the third kind with the minus sign in front of $n$.
} 
(E.13) into (E.11) yields

$$
\begin{aligned}
& P_{e 2}\left(N_{0}\right)=\frac{1}{4}\left[1-\frac{1}{\sqrt{1+\frac{N_{0}}{2 E_{2}^{s}}}}\right]\left[1+\frac{1}{\sqrt{1+\frac{N_{0}}{2 E_{1}^{s}\left(1-\rho^{2}\right)}}}\right]+\frac{1}{4}\left[1-\frac{1}{\sqrt{1+\frac{N_{0}}{2 E_{1}^{s}\left(1-\rho^{2}\right)}}}\right] \\
& -\frac{1}{4}\left(\frac{1}{2 \pi}\right)^{2} \frac{1}{\sqrt{1+\frac{N_{0}}{2 E_{2}^{s}}}} \int_{-\pi}^{\pi}\left[\frac{2}{\sqrt{1+\alpha}} \pi\right] d \theta_{2} \\
& +\frac{1}{4}\left(\frac{1}{2 \pi}\right)^{2} \frac{1}{\sqrt{1+\frac{N_{0}}{2 E_{2}^{s}}}} \int_{-\pi}^{\pi} 4 \frac{\beta}{(1+\alpha) \sqrt{1+\beta^{2}+\alpha}} \prod\left(\frac{\pi}{2}, \frac{\alpha}{1+\alpha}, \sqrt{\frac{\alpha}{1+\alpha+\beta^{2}}}\right) d \theta_{2} \\
& =\frac{1}{4}\left[1-\frac{1}{\sqrt{1+\frac{N_{0}}{2 E_{2}^{s}}}}\right]\left[1+\frac{1}{\sqrt{1+\frac{N_{0}}{2 E_{1}^{s}\left(1-\rho^{2}\right)}}}\right]+\frac{1}{4}\left[1-\frac{1}{\sqrt{1+\frac{N_{0}}{2 E_{1}^{s}\left(1-\rho^{2}\right)}}}\right] \\
& -\frac{1}{4} \frac{1}{2 \pi} \frac{1}{\sqrt{1+\frac{N_{0}}{2 E_{2}^{8}}}}\left[\frac{2}{\sqrt{1+\alpha}} \pi\right] \\
& +\frac{1}{4} \frac{1}{2 \pi} \frac{1}{\sqrt{1+\frac{N_{0}}{2 E_{2}^{s}}}} 4 \frac{\beta}{(1+\alpha) \sqrt{1+\beta^{2}+\alpha}} \prod\left(\frac{\pi}{2}, \frac{\alpha}{1+\alpha}, \sqrt{\frac{\alpha}{1+\alpha+\beta^{2}}}\right) \\
& =\frac{1}{4}\left[1-\frac{1}{\sqrt{1+\frac{N_{0}}{2 E_{2}^{s}}}}\right]\left[1+\frac{1}{\sqrt{1+\frac{N_{0}}{2 E_{1}^{s}\left(1-\rho^{2}\right)}}}\right]+\frac{1}{4}\left[1-\frac{1}{\sqrt{1+\frac{N_{0}}{2 E_{1}^{s}\left(1-\rho^{2}\right)}}}\right] \\
& -\frac{1}{4} \frac{1}{\sqrt{1+\frac{N_{0}}{2 E_{2}^{s}}}} \frac{1}{\sqrt{\left(1+4 \rho^{2} \frac{E_{1}^{s}}{E_{2}^{s}}\right) \frac{1+\frac{N_{0}}{2 E_{2}^{s}+4 \rho^{2} 2 E_{1}^{s}}}{1+\frac{N_{0}}{2 E_{2}^{s}}}}} \\
& +\frac{1}{2 \pi} \frac{1}{\sqrt{1+\frac{N_{0}}{2 E_{2}^{s}}}} \frac{1}{\left(1+4 \rho^{2} \frac{E_{1}^{s}}{E_{2}^{s}}\right) \frac{\sqrt{1+\frac{N_{0}}{2 E_{2}^{s}}}}{\frac{1+\frac{N_{0}}{2 E_{2}^{s}+4 \rho^{2} 2 E_{1}^{s}}}{1+\frac{N_{0}}{2 E_{2}^{s}}}}} \frac{\sqrt{\left.\left(1+\frac{N_{0}}{2 E_{2}^{s}}\right)+\frac{4 \rho^{2} N_{0}}{2 E_{2}^{s}\left(1-\rho^{2}\right)}+\frac{N_{0}\left(1+\frac{N_{0}}{2 E_{2}^{s}}\right)}{2 E_{1}^{s}\left(1-\rho^{2}\right)}\right]^{1 / 2}}}{\left[\begin{array}{ll}
2 \\
(1)
\end{array}\right.} \\
& \cdot \prod\left(\frac{\pi}{2}, \frac{4 \rho^{2} 2 E_{1}^{s}}{\left(2 E_{2}^{s}+4 \rho^{2} 2 E_{1}^{s}\right)\left(1+\frac{N_{0}}{2 E_{2}^{s}+4 \rho^{2} 2 E_{1}^{s}}\right)}, \sqrt{\left.\frac{4 \rho^{2} N_{0}}{\left(1-\rho^{2}\right) 2 E_{2}^{s}} \frac{1}{1+\frac{N_{0}\left[4 \rho^{2}+\left(1-\rho^{2}\right)+\frac{2 E_{2}^{s}}{2 E_{1}^{s}}+\frac{N_{0}}{2 E_{1}^{s}}\right]}{\left(1-\rho^{2}\right) 2 E_{2}^{s}}}\right)}\right.
\end{aligned}
$$




$$
\begin{aligned}
= & \frac{1}{4}\left[1-\frac{1}{\sqrt{1+\frac{N_{0}}{2 E_{2}^{s}}}}\right]\left[1+\frac{1}{\sqrt{1+\frac{N_{0}}{2 E_{1}^{s}\left(1-\rho^{2}\right)}}}\right]+\frac{1}{4}\left[1-\frac{1}{\sqrt{1+\frac{N_{0}}{2 E_{1}^{s}\left(1-\rho^{2}\right)}}}\right] \\
& -\frac{1}{4} \frac{1}{\sqrt{\left(1+4 \rho^{2} \frac{E_{1}^{s}}{E_{2}^{s}}\right)\left(1+\frac{N_{0}}{2 E_{2}^{s}\left(1+4 \rho^{2} \frac{E_{1}^{s}}{E_{2}^{s}}\right)}\right)}} \\
+ & \frac{1}{2 \pi} \frac{1+\frac{N_{0}}{2 E_{2}^{s}}}{\left(1+4 \rho^{2} \frac{E_{1}^{s}}{E_{2}^{s}}\right)\left(1+\frac{N_{0}}{2 E_{2}^{s}\left(1+4 \rho^{2} \frac{E_{1}^{s}}{E_{2}^{s}}\right)}\right)} \frac{1}{\left.\left[1+\frac{N_{0}}{2 E_{2}^{s}}\right)+\frac{4 \rho^{2} N_{0}}{2 E_{2}^{s}\left(1-\rho^{2}\right)}+\frac{N_{0}\left(1+\frac{N_{0}}{2 E_{2}^{s}}\right)}{2 E_{1}^{s}\left(1-\rho^{2}\right)}\right]^{1 / 2}} \\
& \cdot \prod\left(\frac{\pi}{2}, \frac{4 \rho^{2} 2 E_{1}^{s}}{\left(2 E_{2}^{s}+4 \rho^{2} 2 E_{1}^{s}\right)\left(1+\frac{N_{0}}{2 E_{2}^{s}+4 \rho^{2} 2 E_{1}^{s}}\right)}, \sqrt{\left.\frac{4 \rho^{2} N_{0}}{\left(1-\rho^{2}\right) 2 E_{2}^{s}} \frac{1}{1+\frac{N_{0}\left[4 \rho^{2}+\left(1-\rho^{2}\right)+\frac{2 E_{2}^{s}}{2 E_{1}^{s}}+\frac{N_{0}}{2 E_{1}^{s}}\right]}{\left(1-\rho^{2}\right) 2 E_{2}^{s}}}\right)}\right.
\end{aligned}
$$

since

$$
\begin{aligned}
& 1+\alpha=1+\frac{4 \rho^{2} 2 E_{1}^{s}}{N_{0}\left(1+\frac{2 E_{2}^{s}}{N_{0}}\right)}=1+\frac{4 \rho^{2} 2 E_{1}^{s}}{N_{0}+2 E_{2}^{s}}=\frac{N_{0}+2 E_{2}^{s}+4 \rho^{2} 2 E_{1}^{s}}{N_{0}+2 E_{2}^{s}} \\
& =\frac{2 E_{2}^{s}+4 \rho^{2} 2 E_{1}^{s}}{2 E_{2}^{s}} \frac{1+\frac{N_{0}}{2 E_{2}^{s}+4 \rho^{2} 2 E_{1}^{s}}}{1+\frac{N_{0}}{2 E_{2}^{s}}}=\left(1+4 \rho^{2} \frac{E_{1}^{s}}{E_{2}^{s}}\right) \frac{1+\frac{N_{0}}{2 E_{2}^{s}+4 \rho^{2} 2 E_{1}^{s}}}{1+\frac{N_{0}}{2 E_{2}^{s}}} \\
& 1+\alpha+\beta^{2}=1+\frac{4 \rho^{2} 2 E_{1}^{s}}{N_{0}\left(1+\frac{2 E_{2}^{s}}{N_{0}}\right)}+\frac{2 E_{1}^{s}\left(1-\rho^{2}\right)}{N_{0}} \\
& =\frac{2 E_{1}^{s}\left(1-\rho^{2}\right)}{N_{0}}\left[1+\frac{4 \rho^{2} N_{0}}{\left(1-\rho^{2}\right)\left(N_{0}+2 E_{2}^{s}\right)}+\frac{N_{0}}{2 E_{1}^{s}\left(1-\rho^{2}\right)}\right] \\
& \frac{\beta}{\sqrt{1+\alpha+\beta^{2}}}=\frac{1}{\left[1+\frac{4 \rho^{2} N_{0}}{\left(1-\rho^{2}\right)\left(N_{0}+2 E_{2}^{s}\right)}+\frac{N_{0}}{2 E_{1}^{s}\left(1-\rho^{2}\right)}\right]^{1 / 2}}=\frac{\sqrt{N_{0}+2 E_{2}^{s}}}{\left[N_{0}+2 E_{2}^{s}+\frac{4 \rho^{2} N_{0}}{\left(1-\rho^{2}\right)}+\frac{N_{0}\left(N_{0}+2 E_{2}^{s}\right)}{2 E_{1}^{s}\left(1-\rho^{2}\right)}\right]^{1 / 2}} \\
& =\frac{\sqrt{2 E_{2}^{s}\left(1+\frac{N_{0}}{2 E_{2}^{s}}\right)}}{\left[2 E_{2}^{s}\left(1+\frac{N_{0}}{2 E_{2}^{s}}\right)+\frac{4 \rho^{2} N_{0}}{\left(1-\rho^{2}\right)}+\frac{N_{0} 2 E_{2}^{s}\left(1+\frac{N_{0}}{2 E_{2}^{s}}\right)}{2 E_{1}^{s}\left(1-\rho^{2}\right)}\right]^{1 / 2}} \\
& =\frac{\sqrt{1+\frac{N_{0}}{2 E_{2}^{s}}}}{\left[\left(1+\frac{N_{0}}{2 E_{2}^{s}}\right)+\frac{4 \rho^{2} N_{0}}{2 E_{2}^{s}\left(1-\rho^{2}\right)}+\frac{N_{0}\left(1+\frac{N_{0}}{2 E_{2}^{s}}\right)}{2 E_{1}^{s}\left(1-\rho^{2}\right)}\right]^{1 / 2}}
\end{aligned}
$$




$$
\begin{aligned}
\frac{\alpha}{1+\alpha+\beta^{2}} & =\frac{\frac{4 \rho^{2} 2 E_{1}^{s}}{N_{0}\left(1+\frac{2 E_{2}^{s}}{N_{0}}\right)}}{\frac{4 \rho^{2} 2 E_{1}^{s}}{N_{0}\left(1+\frac{2 E_{2}^{s}}{N_{0}}\right)}\left[1+\frac{\left(1-\rho^{2}\right)\left(1+\frac{2 E_{2}^{s}}{N_{0}}\right)}{4 \rho^{2}}+\frac{N_{0}\left(1+\frac{2 E_{2}^{s}}{N_{0}}\right)}{4 \rho^{2} 2 E_{1}^{s}}\right]}=\frac{1}{1+\frac{\left(1-\rho^{2}\right)\left(N_{0}+2 E_{2}^{s}\right)}{4 \rho^{2} N_{0}}+\frac{\left(N_{0}+2 E_{2}^{s}\right)}{4 \rho^{2} 2 E_{1}^{s}}} \\
& =\frac{4 \rho^{2} N_{0}}{4 \rho^{2} N_{0}+\left(1-\rho^{2}\right)\left(N_{0}+2 E_{2}^{s}\right)+\frac{N_{0}\left(N_{0}+2 E_{2}^{s}\right)}{2 E_{1}^{s}}} \\
& =\frac{4 \rho^{2} N_{0}}{\left(1-\rho^{2}\right) 2 E_{2}^{s}+N_{0}\left[4 \rho^{2}+\left(1-\rho^{2}\right)+\frac{2 E_{2}^{s}}{2 E_{1}^{s}}+\frac{N_{0}}{2 E_{1}^{s}}\right]} \\
& =\frac{4 \rho^{2} N_{0}}{\left(1-\rho^{2}\right) 2 E_{2}^{s}} \frac{1}{1+N_{0} \frac{\left(4 \rho^{2}+\left(1-\rho^{2}\right)+\frac{2 E_{2}^{s}}{2 E_{1}^{s}}+\frac{N_{0}}{2 E_{1}^{s}}\right]}{\left(1-\rho^{2}\right) 2 E_{2}^{s}}} \\
\frac{\alpha}{1+\alpha} & =\frac{4 \rho^{2} 2 E_{1}^{s}}{1+\frac{4 \rho^{2} 2 E_{1}^{s}}{N_{0}\left(1+\frac{2 E_{2}^{s}}{N_{0}}\right)}}=\frac{4 \rho^{2} 2 E_{1}^{s}}{N_{0}\left(1+\frac{2 E_{2}^{s}}{N_{0}}\right)} \\
& =\frac{\left.4 \rho_{2}^{2} 2 E_{1}^{s}\right)+4 \rho^{2} 2 E_{1}^{s}}{\left(2 E_{2}^{s}+4 \rho^{2} 2 E_{1}^{s}\right)\left(1+\frac{N_{0}}{2 E_{2}^{s}+4 \rho^{2} 2 E_{1}^{s}}\right)}
\end{aligned}
$$

From (5.19)

$$
\eta_{2}=\lim _{\sigma \rightarrow 0} \frac{\sigma^{2}}{4 E_{2}^{s} P_{e, 2}(\sigma)}=\lim _{N_{0} \rightarrow 0} \frac{N_{0} / 2}{4 E_{2}^{s} P_{e, 2}\left(N_{0}\right)}=\lim _{N_{0} \rightarrow 0} \frac{N_{0}}{8 E_{2}^{s} P_{e, 2}\left(N_{0}\right)}
$$

Let us define

$$
\begin{gathered}
\gamma=\frac{4 \rho^{2} 2 E_{1}^{s}}{\left(2 E_{2}^{s}+4 \rho^{2} 2 E_{1}^{s}\right)} \quad \delta=\frac{1}{2 E_{2}^{s}+4 \rho^{2} 2 E_{1}^{s}} \quad \mu=\frac{4 \rho^{2}}{\left(1-\rho^{2}\right) 2 E_{2}^{s}} \quad \nu=\frac{4 \rho^{2}+\left(1-\rho^{2}\right)+\frac{2 E_{2}^{s}}{2 E_{1}^{s}}}{\left(1-\rho^{2}\right) 2 E_{2}^{s}} \\
\zeta=\frac{1}{\left(1-\rho^{2}\right) 2 E_{2}^{s} 2 E_{1}^{s}}
\end{gathered}
$$


As $N_{0} \rightarrow 0$

$$
\begin{aligned}
& \prod\left(\frac{\pi}{2}, \frac{4 \rho^{2} 2 E_{1}^{s}}{\left(2 E_{2}^{s}+4 \rho^{2} 2 E_{1}^{s}\right)\left(1+\frac{N_{0}}{2 E_{2}^{s}+4 \rho^{2} 2 E_{1}^{s}}\right)}, \sqrt{\left.\frac{4 \rho^{2} N_{0}}{\left(1-\rho^{2}\right) 2 E_{2}^{s}} \frac{1}{1+\frac{N_{0}\left[4 \rho^{2}+\left(1-\rho^{2}\right)+\frac{2 E_{2}^{s}}{2 E_{1}^{s}}+\frac{N_{0}}{2 E_{1}^{s}}\right]}{\left(1-\rho^{2}\right) 2 E_{2}^{s}}}\right)}\right. \\
& =\prod\left(\frac{\pi}{2}, \frac{\gamma}{1+\delta N_{0}}, \sqrt{N_{0} \mu \frac{1}{1+\nu N_{0}+\zeta N_{0}^{2}}}\right) \\
& =\int_{0}^{\frac{\pi}{2}} \frac{d u}{\left(1-\frac{\gamma}{1+\delta N_{0}} \sin ^{2} u\right) \sqrt{1-\frac{N_{0} \mu}{1+\nu N_{0}+\zeta N_{0}^{2}} \sin ^{2} u}} \\
& =\int_{0}^{\frac{\pi}{2}} \frac{\left(1+\delta N_{0}\right) \sqrt{1+\nu N_{0}+\zeta N_{0}^{2}} d u}{\left(1+\delta N_{0}-\gamma \sin ^{2} u\right) \sqrt{1+\nu N_{0}+\zeta N_{0}^{2}-N_{0} \mu \sin ^{2} u}} \\
& =\int_{0}^{\frac{\pi}{2}} \frac{\left(1+\delta N_{0}\right) \sqrt{1+\nu N_{0}+\zeta N_{0}^{2}} d u}{\left(1-\gamma \sin ^{2} u\right)\left(1+\frac{\delta}{1-\gamma \sin ^{2} u} N_{0}\right) \sqrt{1+N_{0}\left(\nu-\mu \sin ^{2} u\right)+\zeta N_{0}^{2}}} \\
& \approx\left(1+\delta N_{0}\right)\left(1+\frac{1}{2} \nu N_{0}\right) \int_{0}^{\frac{\pi}{2}} \frac{\left(1-\frac{\delta}{1-\gamma \sin ^{2} u} N_{0}\right)\left(1-\frac{1}{2} N_{0}\left(\nu-\mu \sin ^{2} u\right)\right) d u}{\left(1-\gamma \sin ^{2} u\right)} \\
& \approx\left(1+\delta N_{0}+\frac{1}{2} \nu N_{0}\right) \int_{0}^{\frac{\pi}{2}}\left(1-\frac{\delta}{1-\gamma \sin ^{2} u} N_{0}-\frac{1}{2} N_{0}\left(\nu-\mu \sin ^{2} u\right)\right) \frac{d u}{\left(1-\gamma \sin ^{2} u\right)} \\
& =\left(1+\delta N_{0}+\frac{1}{2} \nu N_{0}\right) \int_{0}^{\frac{\pi}{2}}\left(\frac{1}{1-\gamma \sin ^{2} u}-N_{0}\left[\frac{\delta}{\left(1-\gamma \sin ^{2} u\right)^{2}}+\frac{1}{2} \frac{\nu-\mu \sin ^{2} u}{1-\gamma \sin ^{2} u}\right]\right) d u \\
& \approx\left(1+\delta N_{0}+\frac{1}{2} \nu N_{0}\right) \int_{0}^{\frac{\pi}{2}} \frac{d u}{1-\gamma \sin ^{2} u}-N_{0} \int_{0}^{\frac{\pi}{2}}\left[\frac{\delta}{\left(1-\gamma \sin ^{2} u\right)^{2}}+\frac{1}{2} \frac{\nu-\mu \sin ^{2} u}{1-\gamma \sin ^{2} u}\right] d u \\
& \approx\left(1+\left[\delta+\frac{1}{2} \nu-\frac{1}{2} \nu\right] N_{0}\right) \int_{0}^{\frac{\pi}{2}} \frac{d u}{1-\gamma \sin ^{2} u}-N_{0} \delta \int_{0}^{\frac{\pi}{2}} \frac{d u}{\left(1-\gamma \sin ^{2} u\right)^{2}} \\
& +\frac{N_{0} \mu}{2} \int_{0}^{\frac{\pi}{2}} \frac{\sin ^{2} u d u}{1-\gamma \sin ^{2} u} \\
& =\left(1+\delta N_{0}\right) \int_{0}^{\frac{\pi}{2}} \frac{d u}{1-\gamma \sin ^{2} u}-N_{0} \delta \int_{0}^{\frac{\pi}{2}} \frac{d u}{\left(1-\gamma \sin ^{2} u\right)^{2}}+\frac{N_{0} \mu}{2} \int_{0}^{\frac{\pi}{2}} \frac{\sin ^{2} u d u}{1-\gamma \sin ^{2} u}
\end{aligned}
$$




$$
\begin{aligned}
& \int_{0}^{\frac{\pi}{2}} \frac{d u}{\left(1-\gamma \sin ^{2} u\right)} d u=\int_{0}^{1} \frac{d x}{\left(1-\gamma x^{2}\right) \sqrt{1-x^{2}}} \quad x=\sin u \\
& =\int_{0}^{1} \frac{d x}{\left(a x^{2}+b\right) \sqrt{c x^{2}+d}} \quad \text { with } a=-\gamma \quad b=1 \quad c=-1 \quad d=1 \\
& =\left[\frac{1}{\sqrt{d(a d-b c)}} \operatorname{tg}^{-1}\left[\frac{x \sqrt{a d-b c}}{\sqrt{b\left(c x^{2}+d\right)}}\right]\right]_{0}^{1}=\left[\frac{1}{\sqrt{-\gamma+1}} \operatorname{tg}^{-1}\left[\frac{x \sqrt{-\gamma+1}}{\sqrt{-x^{2}+1}}\right]\right]_{0}^{1} \\
& =\frac{1}{\sqrt{-\gamma+1}} \operatorname{tg}^{-1}(\infty)=\frac{1}{\sqrt{-\gamma+1}} \frac{\pi}{2}
\end{aligned}
$$

since $\gamma=\frac{4 \rho^{2} 2 E_{1}^{s}}{\left(2 E_{2}^{s}+4 \rho^{2} 2 E_{1}^{s}\right)}<1$ which implies that $a d-b c=-\gamma-(-1)=1-\gamma>0$.

Using equation 3.681 of [135], we obtain

$$
\begin{gathered}
\int_{0}^{\frac{\pi}{2}} \frac{d u}{\left(1-\gamma \sin ^{2} u\right)^{2}}=\int_{0}^{\frac{\pi}{2}} \frac{\sin ^{2 \mu-1} u \cos ^{2 \nu-1} u d u}{\left(1-\gamma \sin ^{2} u\right)^{\rho}} \text { with } \mu=\nu=\frac{1}{2}, \rho=2, \Re(\mu)>0, \Re(\nu)>0 \\
=\frac{1}{2} B(\mu, \nu) F(\rho, \mu ; \mu+\nu ; \gamma)=\frac{1}{2} B\left(\frac{1}{2}, \frac{1}{2}\right) F\left(2, \frac{1}{2} ; 1 ; \gamma\right) \\
=\frac{1}{2} \frac{\Gamma(1 / 2) \Gamma(1 / 2)}{\Gamma(1)} F\left(2, \frac{1}{2} ; 1 ; \gamma\right) \quad \text { using } B(x, y)=\frac{\Gamma(x) \Gamma(y)}{\Gamma(x+y)} \\
=\frac{1}{2} \frac{\pi}{1} F\left(2, \frac{1}{2} ; 1 ; \gamma\right)=\frac{1}{2} \pi F\left(2, \frac{1}{2} ; 1 ; \gamma\right)=\frac{\pi}{2} \frac{2-\gamma}{2(1-\gamma) \sqrt{1-\gamma}}
\end{gathered}
$$

where $B(x, y)$ is the beta function (Euler's integral of the first kind) defined as

$$
B(x, y)=\int_{0}^{1} t^{x-1}(1-t)^{y-1} d t
$$

$\Gamma(z)$ is the Gamma function defined as

$$
\Gamma(z)=\int_{0}^{\infty} e^{-t} t^{z-1} d t
$$

$F(\alpha, \beta ; \gamma ; z)$ is the Gauss hypergeometric function defined as

$$
F(\alpha, \beta ; \gamma ; z)=1+\frac{\alpha \cdot \beta}{\gamma \cdot 1} z+\frac{\alpha(\alpha+1) \beta(\beta+1)}{\gamma(\gamma+1) \cdot 1 \cdot 2} z^{2}+\frac{\alpha(\alpha+1)(\alpha+2) \beta(\beta+1)(\beta+2)}{\gamma(\gamma+1)(\gamma+2) \cdot 1 \cdot 2 \cdot 3} z^{3}+\cdots
$$


which satisfies

$$
\begin{gathered}
F(\alpha, \beta ; \beta ; z)=F(\beta, \alpha ; \beta ; z)=(1-z)^{-\alpha} \\
\text { and } \\
(2 \alpha-\gamma-\alpha z+\beta z) F(\alpha, \beta ; \gamma ; z)+(\gamma-\alpha) F(\alpha-1, \beta ; \gamma ; z)+\alpha(z-1) F(\alpha+1, \beta ; \gamma ; z)=0 \\
\left(2-1-z+\frac{1}{2} z\right) F\left(1, \frac{1}{2} ; 1 ; z\right)+(1-1) F\left(1-1, \frac{1}{2} ; 1 ; z\right)+(z-1) F\left(1+1, \frac{1}{2} ; 1 ; z\right)=0 \\
\left(1-\frac{1}{2} z\right)(1-z)^{-1 / 2}+(z-1) F\left(2, \frac{1}{2} ; 1 ; z\right)=0 \\
F\left(2, \frac{1}{2} ; 1 ; z\right)=-\frac{\left(1-\frac{1}{2} z\right)(1-z)^{-1 / 2}}{z-1}=\frac{2-z}{2 \sqrt{1-z}(1-z)}
\end{gathered}
$$

Using equation 3.615 of [135], we obtain

$$
\begin{aligned}
& \int_{0}^{\frac{\pi}{2}} \frac{\sin ^{2} u d u}{1-\gamma \sin ^{2} u}=\frac{1}{2} \int_{0}^{\frac{\pi}{2}} \frac{1-\cos (2 u)}{1-\gamma \sin ^{2} u} d u=\frac{1}{2}\left[\int_{0}^{\frac{\pi}{2}} \frac{d u}{1-\gamma \sin ^{2} u}-\int_{0}^{\frac{\pi}{2}} \frac{\cos (2 u)}{1-\gamma \sin ^{2} u} d u\right] \\
& =\frac{1}{2}\left[\frac{1}{\sqrt{-\gamma+1}} \frac{\pi}{2}-\int_{0}^{\frac{\pi}{2}} \frac{\cos (2 n u)}{1-\gamma \sin ^{2} u} d u\right] \text { with } n=1 \text { and using } 0 \leq \gamma<1 \\
& =\frac{1}{2}\left[\frac{1}{\sqrt{-\gamma+1}} \frac{\pi}{2}-\frac{(-1)^{n} \pi}{2 \sqrt{1-\gamma}}\left(\frac{1-\sqrt{1-\gamma}}{\sqrt{\gamma}}\right)^{2 n}\right] \\
& =\frac{1}{2}\left[\frac{1}{\sqrt{-\gamma+1}} \frac{\pi}{2}+\frac{\pi}{2 \sqrt{1-\gamma}}\left(\frac{1-\sqrt{1-\gamma}}{\sqrt{\gamma}}\right)^{2}\right] \\
& =\frac{1}{2} \frac{\pi}{2} \frac{1}{\sqrt{1-\gamma}}\left[1+\left(\frac{1-\sqrt{1-\gamma}}{\sqrt{\gamma}}\right)^{2}\right]=\frac{1}{2} \frac{\pi}{2} \frac{1}{\sqrt{1-\gamma}}\left[1+\frac{1-2 \sqrt{1-\gamma}+1-\gamma}{\gamma}\right] \\
& =\frac{1}{2} \frac{\pi}{2} \frac{1}{\sqrt{1-\gamma}}\left[\frac{\gamma+2-2 \sqrt{1-\gamma}-\gamma}{\gamma}\right]=\frac{\pi}{2} \frac{1-\sqrt{1-\gamma}}{\gamma \sqrt{1-\gamma}}
\end{aligned}
$$

Alternate method, using equation 3.681 of [135], we obtain

$$
\begin{aligned}
\int_{0}^{\frac{\pi}{2}} \frac{\sin ^{2} u d u}{1-\gamma \sin ^{2} u} & =\int_{0}^{\frac{\pi}{2}} \frac{\sin ^{2 \mu-1} u \cos ^{2 \nu-1} u d u}{\left(1-\gamma \sin ^{2} u\right)^{\rho}} \text { with } \mu=\frac{3}{2}, \nu=\frac{1}{2}, \rho=1, \Re(\mu)>0, \Re(\nu)>0 \\
& =\frac{1}{2} B(\mu, \nu) F(\rho, \mu ; \mu+\nu ; \gamma)=\frac{1}{2} B\left(\frac{3}{2}, \frac{1}{2}\right) F\left(1, \frac{3}{2} ; 2 ; \gamma\right)
\end{aligned}
$$




$$
\begin{aligned}
& =\frac{1}{2} \frac{\Gamma(3 / 2) \Gamma(1 / 2)}{\Gamma(2)} F\left(1, \frac{3}{2} ; 2 ; \gamma\right)=\frac{1}{2} \frac{\frac{1}{2} \Gamma^{2}(1 / 2)}{1} F\left(1, \frac{3}{2} ; 2 ; \gamma\right) \\
& =\frac{1}{2} \frac{1}{2} \pi F\left(1, \frac{3}{2} ; 2 ; \gamma\right)=\frac{\pi}{2} \frac{1}{2} \frac{F\left(1, \frac{1}{2} ; 1 ; \gamma\right)-F\left(0, \frac{1}{2} ; 1 ; \gamma\right)}{\gamma / 2} \\
& =\frac{\pi}{2} \frac{1}{2} \frac{(1-\gamma)^{-1 / 2}-1}{\gamma / 2}=\frac{\pi}{2} \frac{1}{\sqrt{1-\gamma}} \frac{1-\sqrt{1-\gamma}}{\gamma}
\end{aligned}
$$

using that $F(\alpha, \beta ; \gamma ; z)$ satisfies

$$
\begin{gathered}
\gamma F(\alpha, \beta ; \gamma ; z)-\gamma F(\alpha+1, \beta ; \gamma ; z)+\beta z F(\alpha+1, \beta+1 ; \gamma+1 ; z)=0 \\
F(0, \beta ; \gamma ; z)=1 \quad F(\alpha, \beta ; \beta ; z)=F(\beta, \alpha ; \beta ; z)=(1-z)^{-\alpha}
\end{gathered}
$$

Substituting (E.15), (E.16) and (E.17) into (E.14) yields

$$
\begin{aligned}
& \prod\left(\frac{\pi}{2}, \frac{4 \rho^{2} 2 E_{1}^{s}}{\left(2 E_{2}^{s}+4 \rho^{2} 2 E_{1}^{s}\right)\left(1+\frac{N_{0}}{2 E_{2}^{s}+4 \rho^{2} 2 E_{1}^{s}}\right)}, \sqrt{\frac{4 \rho^{2} N_{0}}{\left(1-\rho^{2}\right) 2 E_{2}^{s}} \frac{1}{1+\frac{N_{0}\left[4 \rho^{2}+\left(1-\rho^{2}\right)+\frac{2 E_{2}^{s}}{2 E_{1}^{s}}+\frac{N_{0}}{2 E_{1}^{s}}\right]}{\left(1-\rho^{2}\right) 2 E_{2}^{s}}}}\right) \\
& \approx\left(1+\delta N_{0}\right) \frac{1}{\sqrt{-\gamma+1}} \frac{\pi}{2}-N_{0} \delta \frac{\pi}{2} \frac{2-\gamma}{2(1-\gamma) \sqrt{1-\gamma}}+\frac{N_{0} \mu}{2} \frac{\pi}{2} \frac{1-\sqrt{1-\gamma}}{\gamma \sqrt{1-\gamma}} \\
& =\frac{1}{\sqrt{-\gamma+1}} \frac{\pi}{2}+N_{0}\left[\delta \frac{1}{\sqrt{-\gamma+1}} \frac{\pi}{2}-\delta \frac{\pi}{2} \frac{2-\gamma}{2(1-\gamma) \sqrt{1-\gamma}}+\frac{\mu}{2} \frac{\pi}{2} \frac{1-\sqrt{1-\gamma}}{\gamma \sqrt{1-\gamma}}\right] \\
& =\frac{\pi}{2} \frac{1}{\sqrt{1-\gamma}}\left[1+N_{0}\left(\delta-\delta \frac{2-\gamma}{2(1-\gamma)}+\frac{\mu}{2} \frac{1-\sqrt{1-\gamma}}{\gamma}\right)\right] \\
& =\frac{\pi}{2} \frac{1}{\sqrt{1-\gamma}}\left[1+N_{0}\left(\frac{-\delta \gamma}{2(1-\gamma)}+\frac{\mu}{2} \frac{1-\sqrt{1-\gamma}}{\gamma}\right)\right] \\
& =\frac{\frac{\pi}{2}}{\sqrt{1-\frac{4 \rho^{2} 2 E_{s}^{s}}{\left(2 E_{2}^{s}+4 \rho^{2} 2 E_{1}^{s}\right)}}}\left[1+N_{0}\left(\frac{-\frac{1}{2 E_{2}^{s}+4 \rho^{2} 2 E_{1}^{s}} \frac{4 \rho^{2} 2 E_{1}^{s}}{\left(2 E_{2}^{s}+4 \rho^{2} 2 E_{1}^{s}\right)}}{2\left(1-\frac{4 \rho^{2} 2 E_{1}^{s}}{\left(2 E_{2}^{s}+4 \rho^{2} 2 E_{1}^{s}\right)}\right)}+\frac{\frac{4 \rho^{2}}{\left(1-\rho^{2}\right) 2 E_{2}^{s}}}{2} \frac{1-\sqrt{1-\frac{4 \rho^{2} 2 E_{1}^{s}}{\left(2 E_{2}^{s}+4 \rho^{2} 2 E_{1}^{s}\right)}}}{\frac{4 \rho^{2} 2 E_{1}^{s}}{\left(2 E_{2}^{s}+4 \rho^{2} 2 E_{1}^{s}\right)}}\right)\right] \\
& =\frac{\pi}{2} \sqrt{\frac{2 E_{2}^{s}+4 \rho^{2} 2 E_{1}^{s}}{2 E_{2}^{s}}}\left[1+N_{0}\left(\frac{-\frac{4 \rho^{2} 2 E_{1}^{s}}{\left(2 E_{2}^{s}+4 \rho^{2} 2 E_{1}^{s}\right)}}{2 \cdot 2 E_{2}^{s}}+\frac{4 \rho^{2}}{2\left(1-\rho^{2}\right) 2 E_{2}^{s}} \frac{1-\sqrt{\frac{2 E_{2}^{s}}{\left(2 E_{2}^{s}+4 \rho^{2} 2 E_{1}^{s}\right)}}}{\frac{4 \rho^{2} 2 E_{1}^{s}}{\left(2 E_{2}^{s}+4 \rho^{2} 2 E_{1}^{s}\right)}}\right)\right]
\end{aligned}
$$




$$
\begin{aligned}
= & \frac{\pi}{2} \sqrt{\frac{2 E_{2}^{s}+4 \rho^{2} 2 E_{1}^{s}}{2 E_{2}^{s}}}\left[1+N_{0}\left(\frac{-4 \rho^{2} 2 E_{1}^{s}}{2 \cdot 2 E_{2}^{s}\left(2 E_{2}^{s}+4 \rho^{2} 2 E_{1}^{s}\right)}+\frac{4 \rho^{2}}{2\left(1-\rho^{2}\right) 2 E_{2}^{s}} \frac{1-\sqrt{\frac{2 E_{2}^{s}}{\left(2 E_{2}^{s}+4 \rho^{2} 2 E_{1}^{s}\right)}}}{\frac{4 \rho^{2} 2 E_{1}^{s}}{\left(2 E_{2}^{s}+4 \rho^{2} 2 E_{1}^{s}\right)}}\right)\right] \\
= & \frac{\pi}{2} \sqrt{\frac{2 E_{2}^{s}+4 \rho^{2} 2 E_{1}^{s}}{2 E_{2}^{s}}} \\
& \cdot\left[1+N_{0}\left\{\frac{-4 \rho^{2} 2 E_{1}^{s}}{2 \cdot 2 E_{2}^{s}\left(2 E_{2}^{s}+4 \rho^{2} 2 E_{1}^{s}\right)}+\frac{2 E_{2}^{s}+4 \rho^{2} 2 E_{1}^{s}}{2\left(1-\rho^{2}\right) 2 E_{2}^{s} \cdot 2 E_{1}^{s}}\left(1-\sqrt{\frac{2 E_{2}^{s}}{\left(2 E_{2}^{s}+4 \rho^{2} 2 E_{1}^{s}\right)}}\right)\right\}\right]
\end{aligned}
$$

$$
\begin{aligned}
& 8 E_{2}^{s} P_{e, 2}\left(N_{0}\right) \approx 2 E_{2}^{s}\left[1-\left(1-\frac{1}{2} \frac{N_{0}}{2 E_{2}^{s}}\right)\right]\left[1+\left(1-\frac{1}{2} \frac{N_{0}}{2 E_{1}^{s}\left(1-\rho^{2}\right)}\right)\right] \\
& +2 E_{2}^{s}\left[1-\left(1-\frac{1}{2} \frac{N_{0}}{2 E_{1}^{s}\left(1-\rho^{2}\right)}\right)\right]-2 E_{2}^{s} \frac{1}{\sqrt{1+4 \rho^{2} \frac{E_{1}^{s}}{E_{2}^{s}}}}\left[1-\frac{1}{2} \frac{N_{0}}{2 E_{2}^{s}\left(1+4 \rho^{2} \frac{E_{1}^{s}}{E_{2}^{s}}\right)}\right] \\
& +\frac{4 E_{2}^{s}}{\pi} \frac{1+\frac{N_{0}}{2 E_{2}^{s}}}{\left(1+4 \rho^{2} \frac{E_{1}^{s}}{E_{2}^{s}}\right)}\left[1-\frac{N_{0}}{2 E_{2}^{s}\left(1+4 \rho^{2} \frac{E_{1}^{s}}{E_{2}^{s}}\right)}\right]\left[1-\frac{1}{2} \frac{N_{0}}{2 E_{2}^{s}}-\frac{1}{2} \frac{4 \rho^{2} N_{0}}{2 E_{2}^{s}\left(1-\rho^{2}\right)}-\frac{1}{2} \frac{N_{0}}{2 E_{1}^{s}\left(1-\rho^{2}\right)}\right] \\
& \cdot \frac{\pi}{2} \sqrt{\frac{2 E_{2}^{s}+4 \rho^{2} 2 E_{1}^{s}}{2 E_{2}^{s}}} \\
& \cdot\left[1+N_{0}\left\{\frac{-4 \rho^{2} 2 E_{1}^{s}}{2 \cdot 2 E_{2}^{s}\left(2 E_{2}^{s}+4 \rho^{2} 2 E_{1}^{s}\right)}+\frac{2 E_{2}^{s}+4 \rho^{2} 2 E_{1}^{s}}{2\left(1-\rho^{2}\right) 2 E_{2}^{s} \cdot 2 E_{1}^{s}}\left(1-\sqrt{\frac{2 E_{2}^{s}}{\left(2 E_{2}^{s}+4 \rho^{2} 2 E_{1}^{s}\right)}}\right)\right\}\right] \\
& \approx 2 E_{2}^{s}\left[\frac{1}{2} \frac{N_{0}}{2 E_{2}^{s}}\right]\left[2-\frac{1}{2} \frac{N_{0}}{2 E_{1}^{s}\left(1-\rho^{2}\right)}\right]+2 E_{2}^{s}\left[\frac{1}{2} \frac{N_{0}}{2 E_{1}^{s}\left(1-\rho^{2}\right)}\right] \\
& -2 E_{2}^{s} \frac{1}{\sqrt{1+4 \rho^{2} \frac{E_{1}^{s}}{E_{2}^{s}}}}\left[1-\frac{1}{2} \frac{N_{0}}{2 E_{2}^{s}\left(1+4 \rho^{2} \frac{E_{1}^{s}}{E_{2}^{s}}\right)}\right] \\
& +\frac{\frac{4 E_{2}^{s}}{\pi} \frac{\pi}{2} \sqrt{\frac{2 E_{2}^{s}+4 \rho^{2} 2 E_{1}^{s}}{2 E_{2}^{s}}}}{\left(1+4 \rho^{2} \frac{E_{1}^{s}}{E_{2}^{s}}\right)}\left[1+\frac{N_{0}}{2 E_{2}^{s}}-\frac{N_{0}}{2 E_{2}^{s}\left(1+4 \rho^{2} \frac{E_{1}^{s}}{E_{2}^{s}}\right)}-\frac{1}{2} \frac{N_{0}}{2 E_{2}^{s}}-\frac{1}{2} \frac{4 \rho^{2} N_{0}}{2 E_{2}^{s}\left(1-\rho^{2}\right)}-\frac{1}{2} \frac{N_{0}}{2 E_{1}^{s}\left(1-\rho^{2}\right)}-\right. \\
& \cdot\left[1+N_{0}\left\{\frac{-4 \rho^{2} 2 E_{1}^{s}}{2 \cdot 2 E_{2}^{s}\left(2 E_{2}^{s}+4 \rho^{2} 2 E_{1}^{s}\right)}+\frac{2 E_{2}^{s}+4 \rho^{2} 2 E_{1}^{s}}{2\left(1-\rho^{2}\right) 2 E_{2}^{s} \cdot 2 E_{1}^{s}}\left(1-\sqrt{\frac{2 E_{2}^{s}}{\left(2 E_{2}^{s}+4 \rho^{2} 2 E_{1}^{s}\right)}}\right)\right\}\right]
\end{aligned}
$$




$$
\begin{aligned}
& \approx 2 E_{2}^{s}\left[\frac{1}{2} \frac{N_{0}}{2 E_{2}^{s}}\right] 2+2 E_{2}^{s}\left[\frac{1}{2} \frac{N_{0}}{2 E_{1}^{s}\left(1-\rho^{2}\right)}\right]-\frac{2 E_{2}^{s}}{\sqrt{1+4 \rho^{2} \frac{E_{1}^{s}}{E_{2}^{s}}}}\left[1-\frac{1}{2} \frac{N_{0}}{2 E_{2}^{s}\left(1+4 \rho^{2} \frac{E_{1}^{s}}{E_{2}^{s}}\right)}\right] \\
& +2 E_{2}^{s} \frac{\sqrt{1+4 \rho^{2} \frac{E_{1}^{s}}{E_{2}^{s}}}}{\left(1+4 \rho^{2} \frac{E_{1}^{s}}{E_{2}^{s}}\right)}\left[1+\frac{1}{2} \frac{N_{0}}{2 E_{2}^{s}}-\frac{N_{0}}{2 E_{2}^{s}\left(1+4 \rho^{2} \frac{E_{1}^{s}}{E_{2}^{s}}\right)}-\frac{1}{2} \frac{4 \rho^{2} N_{0}}{2 E_{2}^{s}\left(1-\rho^{2}\right)}-\frac{1}{2} \frac{N_{0}}{2 E_{1}^{s}\left(1-\rho^{2}\right)}\right] \\
& \cdot\left[1+N_{0}\left\{\frac{-4 \rho^{2} 2 E_{1}^{s}}{2 \cdot 2 E_{2}^{s}\left(2 E_{2}^{s}+4 \rho^{2} 2 E_{1}^{s}\right)}+\frac{2 E_{2}^{s}+4 \rho^{2} 2 E_{1}^{s}}{2\left(1-\rho^{2}\right) 2 E_{2}^{s} \cdot 2 E_{1}^{s}}\left(1-\sqrt{\frac{2 E_{2}^{s}}{\left(2 E_{2}^{s}+4 \rho^{2} 2 E_{1}^{s}\right)}}\right)\right\}\right] \\
& \approx N_{0}+\frac{E_{2}^{s}}{E_{1}^{s}} \frac{N_{0}}{2\left(1-\rho^{2}\right)}-\frac{2 E_{2}^{s}}{\sqrt{1+4 \rho^{2} \frac{E_{1}^{s}}{E_{2}^{s}}}}+\frac{N_{0}}{2\left(1+4 \rho^{2} \frac{E_{1}^{s}}{E_{2}^{s}}\right)^{3 / 2}}+2 E_{2}^{s} \frac{\sqrt{1+4 \rho^{2} \frac{E_{1}^{s}}{E_{2}^{s}}}}{1+4 \rho^{2} \frac{E_{1}^{s}}{E_{2}^{s}}} \\
& +2 E_{2}^{s} \frac{\sqrt{1+4 \rho^{2} \frac{E_{1}^{s}}{E_{2}^{s}}}}{1+4 \rho^{2} \frac{E_{1}^{s}}{E_{2}^{s}}}\left[\frac{1}{2} \frac{N_{0}}{2 E_{2}^{s}}-\frac{N_{0}}{2 E_{2}^{s}\left(1+4 \rho^{2} \frac{E_{1}^{s}}{E_{2}^{s}}\right)}-\frac{1}{2} \frac{4 \rho^{2} N_{0}}{2 E_{2}^{s}\left(1-\rho^{2}\right)}-\frac{1}{2} \frac{N_{0}}{2 E_{1}^{s}\left(1-\rho^{2}\right)}\right. \\
& \left.+N_{0}\left\{\frac{-4 \rho^{2} 2 E_{1}^{s}}{2 \cdot 2 E_{2}^{s}\left(2 E_{2}^{s}+4 \rho^{2} 2 E_{1}^{s}\right)}+\frac{2 E_{2}^{s}+4 \rho^{2} 2 E_{1}^{s}}{2\left(1-\rho^{2}\right) 2 E_{2}^{s} \cdot 2 E_{1}^{s}}\left(1-\sqrt{\frac{2 E_{2}^{s}}{\left(2 E_{2}^{s}+4 \rho^{2} 2 E_{1}^{s}\right)}}\right)\right\}\right] \\
& \frac{8 E_{2}^{s} P_{e, 2}\left(N_{0}\right)}{N_{0}} \approx 1+\frac{E_{2}^{s}}{E_{1}^{s}} \frac{1}{2\left(1-\rho^{2}\right)}-\frac{1}{2\left(1+4 \rho^{2} \frac{E_{1}^{s}}{E_{2}^{s}}\right)^{3 / 2}} \\
& +\frac{1}{\sqrt{1+4 \rho^{2} \frac{E_{1}^{s}}{E_{2}^{s}}}}\left[\frac{1}{2}-\frac{1}{2} \frac{4 \rho^{2}}{\left(1-\rho^{2}\right)}-\frac{1}{2} \frac{E_{2}^{s}}{E_{1}^{s}\left(1-\rho^{2}\right)}\right. \\
& \left.+\frac{-4 \rho^{2} 2 E_{1}^{s}}{2 \cdot\left(2 E_{2}^{s}+4 \rho^{2} 2 E_{1}^{s}\right)}+\frac{2 E_{2}^{s}+4 \rho^{2} 2 E_{1}^{s}}{2\left(1-\rho^{2}\right) \cdot 2 E_{1}^{s}}\left(1-\sqrt{\frac{2 E_{2}^{s}}{\left(2 E_{2}^{s}+4 \rho^{2} 2 E_{1}^{s}\right)}}\right)\right] \\
& \approx 1+\frac{E_{2}^{s}}{E_{1}^{s}} \frac{1}{2\left(1-\rho^{2}\right)}-\frac{1}{2\left(1+4 \rho^{2} \frac{E_{1}^{s}}{E_{2}^{s}}\right)^{3 / 2}} \\
& +\frac{1}{\sqrt{1+4 \rho^{2} \frac{E_{1}^{s}}{E_{2}^{s}}}}\left[\frac{1}{2}-\frac{1}{2} \frac{4 \rho^{2}}{\left(1-\rho^{2}\right)}-\frac{1}{2} \frac{E_{2}^{s}}{E_{1}^{s}\left(1-\rho^{2}\right)}-\frac{1}{2} \frac{4 \rho^{2} \frac{E_{1}^{s}}{E_{2}^{s}}}{1+4 \rho^{2} \frac{E^{s}}{E_{2}^{s}}}\right. \\
& \left.+2 E_{2}^{s} \frac{1+4 \rho^{2} \frac{E_{1}^{s}}{E_{2}^{s}}}{2\left(1-\rho^{2}\right) \cdot 2 E_{1}^{s}}\left(1-\frac{1}{\sqrt{1+4 \rho^{2} \frac{E_{1}^{s}}{E_{2}^{s}}}}\right)\right]
\end{aligned}
$$




$$
\begin{aligned}
= & 1+\frac{E_{2}^{s}}{E_{1}^{s}} \frac{1}{2\left(1-\rho^{2}\right)}-\frac{1}{2\left(1+4 \rho^{2} \frac{E_{1}^{s}}{E_{2}^{s}}\right)^{3 / 2}}-\frac{1}{2} \frac{4 \rho^{2} \frac{E_{1}^{s}}{E_{2}^{s}}}{\left(1+4 \rho^{2} \frac{E_{1}^{s}}{E_{2}^{s}}\right)^{3 / 2}}-\frac{E_{2}^{s}}{E_{1}^{s} 2\left(1-\rho^{2}\right)} \\
& +\frac{1}{\sqrt{1+4 \rho^{2} \frac{E_{1}^{s}}{E_{2}^{s}}}}\left[\frac{1}{2}-\frac{1}{2} \frac{4 \rho^{2}}{\left(1-\rho^{2}\right)}-\frac{1}{2} \frac{E_{2}^{s}}{E_{1}^{s}\left(1-\rho^{2}\right)}+\frac{E_{2}^{s}}{E_{1}^{s}} \frac{1+4 \rho^{2} \frac{E_{1}^{s}}{E_{2}^{s}}}{2\left(1-\rho^{2}\right)}\right] \\
= & 1-\frac{1+4 \rho^{2} \frac{E_{1}^{s}}{E_{2}^{s}}}{2\left(1+4 \rho^{2} \frac{E_{1}^{s}}{E_{2}^{s}}\right)^{3 / 2}}\left[\frac{1}{\sqrt{1+4 \rho^{2} \frac{E_{1}^{s}}{E_{2}^{s}}}}\left[\frac{1}{2}-\frac{4 \rho^{2}}{2} \frac{1}{\left(1-\rho^{2}\right)}-\frac{E_{2}^{s}}{2} \frac{1}{E_{1}^{s}\left(1-\rho^{2}\right)}+2 E_{2}^{s} \frac{1+4 \rho^{2} \frac{E_{1}^{s}}{E_{2}^{s}}}{2\left(1-\rho^{2}\right) \cdot 2 E_{1}^{s}}\right]\right. \\
= & 1+\frac{1}{\sqrt{1+4 \rho^{2} \frac{E_{1}^{s}}{E_{2}^{s}}}}\left[-\frac{1}{2} \frac{4 \rho^{2}+\frac{E_{2}^{s}}{E_{1}^{s}}}{\left(1-\rho^{2}\right)}+\frac{E_{2}^{s}}{E_{1}^{s}} \frac{1+4 \rho^{2} \frac{E_{1}^{s}}{E_{2}^{s}}}{2\left(1-\rho^{2}\right)}\right]=1
\end{aligned}
$$

Hence

$$
\eta_{2}=\lim _{N_{0} \rightarrow 0} \frac{N_{0}}{8 E_{2}^{s} P_{e, 2}\left(N_{0}\right)}=1
$$

\section{AME of DBSIC for Rayleigh channels (alternate method)}

From (5.19)

$$
\begin{aligned}
\eta_{2} & =\lim _{\sigma \rightarrow 0} \frac{\sigma^{2}}{4 E_{2}^{s} P_{e, 2}(\sigma)}=\lim _{N_{0} \rightarrow 0} \frac{N_{0} / 2}{4 E_{2}^{s} P_{e, 2}\left(N_{0}\right)}=\lim _{N_{0} \rightarrow 0} \frac{N_{0}}{8 E_{2}^{s} P_{e, 2}\left(N_{0}\right)}=\left[8 E_{2}^{s} \lim _{N_{0} \rightarrow 0} \frac{P_{e, 2}\left(N_{0}\right)}{N_{0}}\right]^{-1} \\
& =\left[\left.8 E_{2}^{s} \frac{\partial P_{e, 2}\left(N_{0}\right)}{\partial N_{0}}\right|_{N_{0}=0}\right]^{-1}
\end{aligned}
$$




$$
\begin{aligned}
& \frac{\partial P_{e, 2}\left(N_{0}\right)}{\partial N_{0}}=\frac{\partial}{\partial N_{0}}\left\{\frac{1}{4}\left[1-\frac{1}{\sqrt{1+\frac{N_{0}}{2 E_{2}^{s}}}}\right]\left[1+\frac{1}{\sqrt{1+\frac{N_{0}}{2 E_{1}^{s}\left(1-\rho^{2}\right)}}}\right]\right\} \\
& +\frac{\partial}{\partial N_{0}}\left\{\frac{1}{4}\left[1-\frac{1}{\sqrt{1+\frac{N_{0}}{2 E_{1}^{s}\left(1-\rho^{2}\right)}}}\right]\right\} \\
& -\frac{\partial}{\partial N_{0}}\left\{\frac{1}{4} \frac{1}{\sqrt{\left(1+4 \rho^{2} \frac{E_{1}^{s}}{E_{2}^{s}}\right)\left(1+\frac{N_{0}}{2 E_{2}^{s}\left(1+4 \rho^{2} \frac{E_{1}^{s}}{E_{2}^{s}}\right)}\right)}}\right\} \\
& +\frac{\partial}{\partial N_{0}}\left\{\frac{1}{2 \pi} \frac{1+\frac{N_{0}}{2 E_{2}^{s}}}{\left(1+4 \rho^{2} \frac{E_{1}^{s}}{E_{2}^{s}}\right)\left(1+\frac{N_{0}}{2 E_{2}^{s}\left(1+4 \rho^{2} \frac{E_{1}^{s}}{E_{2}^{s}}\right)}\right)} \frac{1}{\left[\left(1+\frac{N_{0}}{2 E_{2}^{s}}\right)+\frac{4 \rho^{2} N_{0}}{2 E_{2}^{s}\left(1-\rho^{2}\right)}+\frac{N_{0}\left(1+\frac{N_{0}}{2 E_{2}^{s}}\right)}{2 E_{1}^{s}\left(1-\rho^{2}\right)}\right]^{1 / 2}}\right. \\
& \cdot \prod\left(\frac{\pi}{2}, \frac{4 \rho^{2} 2 E_{1}^{s}}{\left(2 E_{2}^{s}+4 \rho^{2} 2 E_{1}^{s}\right)\left(1+\frac{N_{0}}{2 E_{2}^{s}+4 \rho^{2} 2 E_{1}^{s}}\right)}, \sqrt{\left.\frac{4 \rho^{2} N_{0}}{\left(1-\rho^{2}\right) 2 E_{2}^{s}} \frac{1}{1+\frac{N_{0}\left[4 \rho^{2}+\left(1-\rho^{2}\right)+\frac{2 E_{2}^{s}}{2 E_{1}^{s}}+\frac{N_{0}}{2 E_{1}^{s}}\right]}{\left(1-\rho^{2}\right) 2 E_{2}^{s}}}\right)}\right\} \\
& +\frac{1}{2 \pi} \frac{1+\frac{N_{0}}{2 E_{2}^{s}}}{\left(1+4 \rho^{2} \frac{E_{1}^{s}}{E_{2}^{s}}\right)\left(1+\frac{N_{0}}{2 E_{2}^{s}\left(1+4 \rho^{2} \frac{E_{1}^{s}}{E_{2}^{s}}\right)}\right)} \frac{1}{\left[\left(1+\frac{N_{0}}{2 E_{2}^{s}}\right)+\frac{4 \rho^{2} N_{0}}{2 E_{2}^{s}\left(1-\rho^{2}\right)}+\frac{N_{0}\left(1+\frac{N_{0}}{2 E_{2}^{s}}\right)}{2 E_{1}^{s}\left(1-\rho^{2}\right)}\right]^{1 / 2}} \\
& \cdot \prod^{\prime}\left(\frac{\pi}{2}, \frac{4 \rho^{2} 2 E_{1}^{s}}{\left(2 E_{2}^{s}+4 \rho^{2} 2 E_{1}^{s}\right)\left(1+\frac{N_{0}}{2 E_{2}^{s}+4 \rho^{2} 2 E_{1}^{s}}\right)}, \sqrt{\left.\frac{4 \rho^{2} N_{0}}{\left(1-\rho^{2}\right) 2 E_{2}^{s}} \frac{1}{1+\frac{N_{0}\left[4 \rho^{2}+\left(1-\rho^{2}\right)+\frac{2 E_{2}^{s}}{2 E_{1}^{s}}+\frac{N_{0}}{2 E_{1}^{s}}\right]}{\left(1-\rho^{2}\right) 2 E_{2}^{s}}}\right)}\right)
\end{aligned}
$$


where $\Pi^{\prime}(\cdot, \cdot, \cdot)$ denotes the partial derivative of $\Pi(\cdot, \cdot, \cdot)$ with respect to $N_{0}$.

$$
\begin{aligned}
& \frac{\partial P_{e, 2}\left(N_{0}\right)}{\partial N_{0}}=\frac{1}{4}\left[-\left(-\frac{1}{2}\right) \frac{1}{2 E_{2}^{s}}\left(1+\frac{N_{0}}{2 E_{2}^{s}}\right)^{-3 / 2}\right]\left[1+\frac{1}{\sqrt{1+\frac{N_{0}}{2 E_{1}^{s}\left(1-\rho^{2}\right)}}}\right] \\
& +\frac{1}{4}\left[1-\frac{1}{\sqrt{1+\frac{N_{0}}{2 E_{2}^{s}}}}\right]\left[-\frac{1}{2} \frac{1}{2 E_{1}^{s}\left(1-\rho^{2}\right)}\left(1+\frac{N_{0}}{2 E_{1}^{s}\left(1-\rho^{2}\right)}\right)^{-3 / 2}\right] \\
& -\frac{1}{4}\left(-\frac{1}{2}\right) \frac{1}{2 E_{1}^{s}\left(1-\rho^{2}\right)}\left(1+\frac{N_{0}}{2 E_{1}^{s}\left(1-\rho^{2}\right)}\right)^{-3 / 2} \\
& -\frac{1}{4} \frac{1}{\sqrt{\left(1+4 \rho^{2} \frac{E_{1}^{s}}{E_{2}^{s}}\right)}}\left(-\frac{1}{2}\right) \frac{1}{2 E_{2}^{s}\left(1+4 \rho^{2} \frac{E_{s}^{s}}{E_{2}^{s}}\right)}\left(1+\frac{N_{0}}{2 E_{2}^{s}\left(1+4 \rho^{2} \frac{E_{1}^{s}}{E_{2}^{s}}\right)}\right)^{-3 / 2} \\
& +\frac{\partial}{\partial N_{0}}\left\{\frac{1}{2 \pi} \frac{1+\frac{N_{0}}{2 E_{2}^{s}}}{\left(1+4 \rho^{2} \frac{E_{1}^{s}}{E_{2}^{s}}\right)\left(1+\frac{N_{0}}{2 E_{2}^{s}\left(1+4 \rho^{2} \frac{E_{1}^{s}}{E_{2}^{s}}\right)}\right)} \frac{1}{\left[\left(1+\frac{N_{0}}{2 E_{2}^{s}}\right)+\frac{4 \rho^{2} N_{0}}{2 E_{2}^{s}\left(1-\rho^{2}\right)}+\frac{N_{0}\left(1+\frac{N_{0}}{2 E_{2}^{s}}\right)}{2 E_{1}^{s}\left(1-\rho^{2}\right)}\right]^{1 / 2}}\right\} \\
& \cdot \prod\left(\frac{\pi}{2}, \frac{4 \rho^{2} 2 E_{1}^{s}}{\left(2 E_{2}^{s}+4 \rho^{2} 2 E_{1}^{s}\right)\left(1+\frac{N_{0}}{2 E_{2}^{s}+4 \rho^{2} 2 E_{1}^{s}}\right)}, \sqrt{\frac{4 \rho^{2} N_{0}}{\left(1-\rho^{2}\right) 2 E_{2}^{s}} \frac{1}{1+\frac{N_{0}\left[4 \rho^{2}+\left(1-\rho^{2}\right)+\frac{2 E_{s}^{s}}{2 E_{1}^{s}}+\frac{N_{0}}{2 E_{1}^{s}}\right]}{\left(1-\rho^{2}\right) 2 E_{2}^{s}}}}\right) \\
& +\frac{1}{2 \pi} \frac{1+\frac{N_{0}}{2 E_{2}^{s}}}{\left(1+4 \rho^{2} \frac{E^{s}}{E_{2}^{s}}\right)\left(1+\frac{N_{0}}{2 E_{2}^{s}\left(1+4 \rho^{2} \frac{E_{1}^{s}}{E_{2}^{s}}\right)}\right)} \frac{1}{\left[\left(1+\frac{N_{0}}{2 E_{2}^{s}}\right)+\frac{4 \rho^{2} N_{0}}{2 E_{2}^{s}\left(1-\rho^{2}\right)}+\frac{N_{0}\left(1+\frac{N_{0}}{2 E_{2}^{s}}\right)}{2 E_{1}^{s}\left(1-\rho^{2}\right)}\right]^{1 / 2}} \\
& \cdot \prod^{\prime}\left(\frac{\pi}{2}, \frac{4 \rho^{2} 2 E_{1}^{s}}{\left(2 E_{2}^{s}+4 \rho^{2} 2 E_{1}^{s}\right)\left(1+\frac{N_{0}}{2 E_{2}^{s}+4 \rho^{2} 2 E_{1}^{s}}\right)}, \sqrt{\left.\frac{4 \rho^{2} N_{0}}{\left(1-\rho^{2}\right) 2 E_{2}^{s}} \frac{1}{1+\frac{N_{0}\left[4 \rho^{2}+\left(1-\rho^{2}\right)+\frac{2 E_{2}^{s}}{2 E_{1}^{s}}+\frac{N_{0}}{2 E_{1}^{s}}\right]}{\left(1-\rho^{2}\right) 2 E_{2}^{s}}}\right)}\right)
\end{aligned}
$$




$$
\begin{aligned}
& \frac{\partial}{\partial N_{0}}\left\{\frac{1}{2 \pi} \frac{1+\frac{N_{0}}{2 E_{2}^{s}}}{\left(1+4 \rho^{2} \frac{E_{1}^{s}}{E_{2}^{s}}\right)\left(1+\frac{N_{0}}{2 E_{2}^{s}\left(1+4 \rho^{2} \frac{E_{1}^{s}}{E_{2}^{2}}\right)}\right)} \frac{1}{\left[\left(1+\frac{N_{0}}{2 E_{2}^{s}}\right)+\frac{4 \rho^{2} N_{0}}{2 E_{2}^{s}\left(1-\rho^{2}\right)}+\frac{N_{0}\left(1+\frac{N_{0}}{2 E_{2}^{s}}\right)}{2 E_{1}^{s}\left(1-\rho^{2}\right)}\right]^{1 / 2}}\right\} \\
& =\frac{1}{2 \pi} \frac{1}{\left(1+4 \rho^{2} \frac{E_{1}^{s}}{E_{2}^{s}}\right)}\left[\frac{1}{2 E_{2}^{s}} \frac{1}{\left(1+\frac{N_{0}}{2 E_{2}^{s}\left(1+4 \rho^{2} \frac{E_{1}^{s}}{E_{2}^{2}}\right)}\right)} \frac{1}{\left[\left(1+\frac{N_{0}}{2 E_{2}^{s}}\right)+\frac{4 \rho^{2} N_{0}}{2 E_{2}^{s}\left(1-\rho^{2}\right)}+\frac{N_{0}\left(1+\frac{N_{0}}{2 E_{2}^{s}}\right)}{2 E_{1}^{s}\left(1-\rho^{2}\right)}\right]^{1 / 2}}\right. \\
& +\left(1+\frac{N_{0}}{2 E_{2}^{s}}\right)(-1) \frac{1}{2 E_{2}^{s}\left(1+4 \rho^{2} \frac{E_{1}^{s}}{E_{2}^{s}}\right)}\left(1+\frac{N_{0}}{2 E_{2}^{s}\left(1+4 \rho^{2} \frac{E_{1}^{s}}{E_{2}^{s}}\right)}\right)^{-2} \\
& 1 \\
& \cdot \overline{\left[\left(1+\frac{N_{0}}{2 E_{2}^{s}}\right)+\frac{4 \rho^{2} N_{0}}{2 E_{2}^{s}\left(1-\rho^{2}\right)}+\frac{N_{0}\left(1+\frac{N_{0}}{2 E_{2}^{2}}\right)}{2 E_{1}^{s}\left(1-\rho^{2}\right)}\right]^{1 / 2}} \\
& +\frac{\left(1+\frac{N_{0}}{2 E_{2}^{s}}\right)}{\left(1+\frac{N_{0}}{2 E_{2}^{s}\left(1+4 \rho^{2} \frac{E_{1}^{s}}{E_{2}^{2}}\right)}\right)}\left(-\frac{1}{2}\right)\left[\frac{1}{2 E_{2}^{s}}+\frac{4 \rho^{2}}{2 E_{2}^{s}\left(1-\rho^{2}\right)}+\frac{1}{2 E_{1}^{s}\left(1-\rho^{2}\right)}+\frac{2 N_{0}}{2 E_{2}^{s} 2 E_{1}^{s}\left(1-\rho^{2}\right)}\right] \\
& \left.\cdot\left[\left(1+\frac{N_{0}}{2 E_{2}^{s}}\right)+\frac{4 \rho^{2} N_{0}}{2 E_{2}^{s}\left(1-\rho^{2}\right)}+\frac{N_{0}\left(1+\frac{N_{0}}{2 E_{2}^{s}}\right)}{2 E_{1}^{s}\left(1-\rho^{2}\right)}\right]^{-3 / 2}\right] \\
& \prod^{\prime}\left(\frac{\pi}{2}, \frac{4 \rho^{2} 2 E_{1}^{s}}{\left(2 E_{2}^{s}+4 \rho^{2} 2 E_{1}^{s}\right)\left(1+\frac{N_{0}}{2 E_{2}^{s}+4 \rho^{2} 2 E_{1}^{s}}\right)}, \sqrt{\left.\frac{4 \rho^{2} N_{0}}{\left(1-\rho^{2}\right) 2 E_{2}^{s}} \frac{1}{1+\frac{N_{0}\left[4 \rho^{2}+\left(1-\rho^{2}\right)+\frac{2 E_{s}^{s}}{2 E_{1}^{s}}+\frac{N_{0}}{2 E_{1}^{s}}\right]}{\left(1-\rho^{2}\right) 2 E_{2}^{s}}}\right)}\right) \\
& =\frac{\partial}{\partial N_{0}}\left\{\prod\left(\frac{\pi}{2}, \frac{\gamma}{1+\delta N_{0}}, \sqrt{N_{0} \mu \frac{1}{1+\nu N_{0}+\zeta N_{0}^{2}}}\right)\right\} \\
& =\frac{\partial}{\partial N_{0}}\left\{\int_{0}^{\frac{\pi}{2}} \frac{d u}{\left(1-\frac{\gamma}{1+\delta N_{0}} \sin ^{2} u\right) \sqrt{1-\frac{N_{0} \mu}{1+\nu N_{0}+\zeta N_{0}^{2}} \sin ^{2} u}}\right\}
\end{aligned}
$$




$$
\begin{aligned}
= & \int_{0}^{\frac{\pi}{2}} \frac{\partial}{\partial N_{0}}\left\{\frac{1}{\left(1-\frac{\gamma}{1+\delta N_{0}} \sin ^{2} u\right) \sqrt{1-\frac{N_{0} \mu}{1+\nu N_{0}+\zeta N_{0}^{2}} \sin ^{2} u}}\right\} d u \\
= & \int_{0}^{\frac{\pi}{2}}\left\{\frac{(-1)\left[(-1) \gamma\left(\sin ^{2} u\right)(-1) \delta\left(1+\delta N_{0}\right)^{-2}\right]\left(1-\frac{\gamma}{1+\delta N_{0}} \sin ^{2} u\right)^{-2}}{\sqrt{1-\frac{N_{0} \mu}{1+\nu N_{0}+\zeta N_{0}^{2}} \sin ^{2} u}}\right. \\
& \left.+\frac{\left(-\frac{1}{2}\right)\left[-\sin ^{2} u \frac{\partial}{\partial N_{0}}\left[\frac{N_{0} \mu}{1+\nu N_{0}+\zeta N_{0}^{2}}\right]\right]\left(1-\frac{N_{0} \mu}{1+\nu N_{0}+\zeta N_{0}^{2}} \sin ^{2} u\right)^{-3 / 2}}{\left(1-\frac{\gamma}{1+\delta N_{0}} \sin ^{2} u\right)}\right\} d u \\
= & \int_{0}^{\frac{\pi}{2}}\left\{-\gamma \delta\left(\sin ^{2} u\right)\left(1+\delta N_{0}\right)^{-2}\left(1-\frac{\gamma}{1+\delta N_{0}} \sin ^{2} u\right)^{-2} \frac{1}{\sqrt{1-\frac{N_{0} \mu}{1+\nu N_{0}+\zeta N_{0}^{2}} \sin ^{2} u}}\right. \\
+ & \frac{(1 / 2) \sin ^{2} u}{\left(1-\frac{\gamma}{1+\delta N_{0}} \sin ^{2} u\right)}\left[\mu\left(1+\nu N_{0}+\zeta N_{0}^{2}\right)^{-1}+N_{0} \mu(-1)\left(\nu+2 \zeta N_{0}\right)\left(1+\nu N_{0}+\zeta N_{0}^{2}\right)^{-2}\right] \\
& \left.\cdot\left(1-\frac{N_{0} \mu}{1+\nu N_{0}+\zeta N_{0}^{2}} \sin ^{2} u\right)^{-3 / 2}\right\} d u
\end{aligned}
$$

$$
\begin{aligned}
& \left.\frac{\partial P_{e, 2}\left(N_{0}\right)}{\partial N_{0}}\right|_{N_{0}=0} \\
& =\frac{1}{4}\left[-\left(-\frac{1}{2}\right) \frac{1}{2 E_{2}^{s}}\right] \cdot 2-\frac{1}{4}\left(-\frac{1}{2}\right) \frac{1}{2 E_{1}^{s}\left(1-\rho^{2}\right)}-\frac{1}{4} \frac{1}{\sqrt{\left(1+4 \rho^{2} \frac{E_{1}^{s}}{E_{2}^{s}}\right)}}\left(-\frac{1}{2}\right) \frac{1}{2 E_{2}^{s}\left(1+4 \rho^{2} \frac{E_{1}^{s}}{E_{2}^{s}}\right)} \\
& +\frac{1}{2 \pi} \frac{1}{\left(1+4 \rho^{2} \frac{E_{1}^{s}}{E_{2}^{s}}\right)}\left[\frac{1}{2 E_{2}^{s}}+(-1) \frac{1}{2 E_{2}^{s}\left(1+4 \rho^{2} \frac{E_{1}^{s}}{E_{2}^{s}}\right)} \cdot 1\right. \\
& \left.+\left(-\frac{1}{2}\right)\left[\frac{1}{2 E_{2}^{s}}+\frac{4 \rho^{2}}{2 E_{2}^{s}\left(1-\rho^{2}\right)}+\frac{1}{2 E_{1}^{s}\left(1-\rho^{2}\right)}\right]\right] \cdot \prod\left(\frac{\pi}{2}, \frac{4 \rho^{2} 2 E_{1}^{s}}{\left(2 E_{2}^{s}+4 \rho^{2} 2 E_{1}^{s}\right)}, 0\right) \\
& +\frac{1}{2 \pi} \frac{1}{\left(1+4 \rho^{2} \frac{E_{1}^{s}}{E_{2}^{s}}\right)} \cdot\left\{\int_{0}^{\frac{\pi}{2}}\left[-\gamma \delta\left(\sin ^{2} u\right)\left(1-\gamma \sin ^{2} u\right)^{-2}+\frac{(1 / 2) \sin ^{2} u}{\left(1-\gamma \sin ^{2} u\right)}[\mu] \cdot 1\right] d u\right\}
\end{aligned}
$$




$$
\begin{aligned}
& =\frac{1}{8 E_{2}^{s}}+\frac{1}{8} \frac{1}{2 E_{1}^{s}\left(1-\rho^{2}\right)}+\frac{1}{16 E_{2}^{s}} \frac{1}{\left(1+4 \rho^{2} \frac{E_{1}^{s}}{E_{2}^{s}}\right)^{3 / 2}} \\
& +\frac{1}{2 \pi} \frac{1}{\left(1+4 \rho^{2} \frac{E_{1}^{s}}{E_{2}^{s}}\right)}\left[\frac{1}{2 E_{2}^{s}}-\frac{1}{2 E_{2}^{s}\left(1+4 \rho^{2} \frac{E_{1}^{s}}{E_{2}^{s}}\right)}\right. \\
& \left.+\frac{1}{2}\left[\frac{1}{2 E_{2}^{s}}+\frac{4 \rho^{2}}{2 E_{2}^{s}\left(1-\rho^{2}\right)}+\frac{1}{2 E_{1}^{s}\left(1-\rho^{2}\right)}\right]\right] \cdot \prod\left(\frac{\pi}{2}, \gamma, 0\right) \\
& +\frac{1}{2 \pi} \frac{1}{\left(1+4 \rho^{2} \frac{E_{1}^{s}}{E_{2}^{s}}\right)} \cdot\left\{-\gamma \delta \int_{0}^{\frac{\pi}{2}} \frac{\sin ^{2} u}{\left(1-\gamma \sin ^{2} u\right)^{2}} d u+\frac{\mu}{2} \int_{0}^{\frac{\pi}{2}} \frac{\sin ^{2} u}{\left(1-\gamma \sin ^{2} u\right)} d u\right\}
\end{aligned}
$$

$$
\begin{aligned}
\prod\left(\frac{\pi}{2}, \gamma, 0\right) & =\int_{0}^{\frac{\pi}{2}} \frac{d u}{1-\gamma \sin ^{2} u}=\frac{\pi}{2} \frac{1}{\sqrt{1-\gamma}} \\
\int_{0}^{\frac{\pi}{2}} \frac{\sin ^{2} u}{1-\gamma \sin ^{2} u} d u & =\frac{\pi}{2} \frac{1-\sqrt{1-\gamma}}{\gamma \sqrt{1-\gamma}} \\
\int_{0}^{\frac{\pi}{2}} \frac{\sin ^{2} u}{\left(1-\gamma \sin ^{2} u\right)^{2}} d u & =\frac{1}{\gamma} \int_{0}^{\frac{\pi}{2}} \frac{\gamma \sin ^{2} u}{\left(1-\gamma \sin ^{2} u\right)^{2}} d u=\frac{1}{\gamma} \int_{0}^{\frac{\pi}{2}} \frac{\gamma \sin ^{2} u-1+1}{\left(1-\gamma \sin ^{2} u\right)^{2}} d u \\
& =\frac{1}{\gamma}\left[\int_{0}^{\frac{\pi}{2}} \frac{\gamma \sin ^{2} u-1}{\left(1-\gamma \sin ^{2} u\right)^{2}} d u+\int_{0}^{\frac{\pi}{2}} \frac{1}{\left(1-\gamma \sin ^{2} u\right)^{2}} d u\right] \\
& =\frac{1}{\gamma}\left[-\int_{0}^{\frac{\pi}{2}} \frac{1}{1-\gamma \sin ^{2} u} d u+\int_{0}^{\frac{\pi}{2}} \frac{1}{\left(1-\gamma \sin ^{2} u\right)^{2}} d u\right] \\
& =\frac{1}{\gamma}\left[-\frac{\pi}{2} \frac{1}{\sqrt{1-\gamma}}+\frac{\pi}{2} \frac{2-\gamma}{2(1-\gamma) \sqrt{1-\gamma}}\right] \\
& =\frac{\pi}{2} \frac{1}{\gamma \sqrt{1-\gamma}}\left[-1+\frac{2-\gamma}{2(1-\gamma)}\right]=\frac{\pi}{2} \frac{1}{\gamma \sqrt{1-\gamma}}\left[\frac{-2+2 \gamma+2-\gamma}{2(1-\gamma)}\right] \\
& =\frac{\pi}{2} \frac{1}{\gamma \sqrt{1-\gamma}}\left[\frac{\gamma}{2(1-\gamma)}\right]=\frac{\pi}{2} \frac{1}{2(1-\gamma) \sqrt{1-\gamma}}
\end{aligned}
$$




$$
\begin{aligned}
& \left.\frac{\partial P_{e, 2}\left(N_{0}\right)}{\partial N_{0}}\right|_{N_{0}=0} \\
& =\frac{1}{8 E_{2}^{s}}+\frac{1}{8} \frac{1}{2 E_{1}^{s}\left(1-\rho^{2}\right)}+\frac{1}{16 E_{2}^{s}} \frac{1}{\left(1+4 \rho^{2} \frac{E_{1}^{s}}{E_{2}^{s}}\right)^{3 / 2}} \\
& +\frac{1}{2 \pi} \frac{1}{\left(1+4 \rho^{2} \frac{E_{1}^{s}}{E_{2}^{s}}\right)}\left[\frac{1}{2 E_{2}^{s}}-\frac{1}{2 E_{2}^{s}\left(1+4 \rho^{2} \frac{E_{1}^{s}}{E_{2}^{s}}\right)}\right. \\
& \left.-\frac{1}{2}\left[\frac{1}{2 E_{2}^{s}}+\frac{4 \rho^{2}}{2 E_{2}^{s}\left(1-\rho^{2}\right)}+\frac{1}{2 E_{1}^{s}\left(1-\rho^{2}\right)}\right]\right] \cdot \Pi\left(\frac{\pi}{2}, \gamma, 0\right) \\
& +\frac{1}{2 \pi} \frac{1}{\left(1+4 \rho^{2} \frac{E_{1}^{s}}{E_{2}^{s}}\right)} \cdot\left\{-\gamma \delta \int_{0}^{\frac{\pi}{2}} \frac{\sin ^{2} u}{\left(1-\gamma \sin ^{2} u\right)^{2}} d u+\frac{\mu}{2} \int_{0}^{\frac{\pi}{2}} \frac{\sin ^{2} u}{\left(1-\gamma \sin ^{2} u\right)} d u\right\} \\
& =\frac{1}{8 E_{2}^{s}}+\frac{1}{8} \frac{1}{2 E_{1}^{s}\left(1-\rho^{2}\right)}+\frac{1}{16 E_{2}^{s}} \frac{1}{\left(1+4 \rho^{2} \frac{E_{1}^{s}}{E_{2}^{s}}\right)^{3 / 2}} \\
& +\frac{1}{2 \pi} \frac{1}{\left(1+4 \rho^{2} \frac{E_{1}^{s}}{E_{2}^{s}}\right)}\left[\frac{1}{4 E_{2}^{s}}-\frac{1}{2 E_{2}^{s}\left(1+4 \rho^{2} \frac{E_{1}^{s}}{E_{2}^{s}}\right)}-\frac{4 \rho^{2}}{4 E_{2}^{s}\left(1-\rho^{2}\right)}-\frac{1}{4 E_{1}^{s}\left(1-\rho^{2}\right)}\right] \cdot \frac{\pi}{2} \frac{1}{\sqrt{1-\gamma}} \\
& +\frac{1}{2 \pi} \frac{1}{\left(1+4 \rho^{2} \frac{E_{1}^{s}}{E_{2}^{s}}\right)} \cdot\left\{-\gamma \delta \frac{\pi}{2} \frac{1}{2(1-\gamma) \sqrt{1-\gamma}}+\frac{\mu}{2} \frac{\pi}{2} \frac{1-\sqrt{1-\gamma}}{\gamma \sqrt{1-\gamma}}\right\} \\
& =\frac{1}{8 E_{2}^{s}}+\frac{1}{8} \frac{1}{2 E_{1}^{s}\left(1-\rho^{2}\right)}+\frac{1}{16 E_{2}^{s}} \frac{1}{\left(1+4 \rho^{2} \frac{E_{1}^{s}}{E_{2}^{s}}\right)^{3 / 2}} \\
& +\frac{1}{4} \frac{1}{\left(1+4 \rho^{2} \frac{E_{1}^{s}}{E_{2}^{s}}\right) \sqrt{1-\gamma}}\left[\frac{1}{4 E_{2}^{s}}-\frac{1}{2 E_{2}^{s}\left(1+4 \rho^{2} \frac{E_{1}^{s}}{E_{2}^{s}}\right)}-\frac{4 \rho^{2}}{4 E_{2}^{s}\left(1-\rho^{2}\right)}-\frac{1}{4 E_{1}^{s}\left(1-\rho^{2}\right)}\right. \\
& \left.-\gamma \delta \frac{1}{2(1-\gamma)}+\frac{\mu}{2} \frac{1-\sqrt{1-\gamma}}{\gamma}\right]
\end{aligned}
$$




$$
\begin{aligned}
& =\frac{1}{8 E_{2}^{s}}+\frac{1}{8} \frac{1}{2 E_{1}^{s}\left(1-\rho^{2}\right)}+\frac{1}{16 E_{2}^{s}} \frac{1}{\left(1+4 \rho^{2} \frac{E_{1}^{s}}{E_{2}^{s}}\right)^{3 / 2}} \\
& +\frac{1}{4} \frac{1}{\left(1+4 \rho^{2} \frac{E_{1}^{s}}{E_{2}^{s}}\right)^{1 / 2}}\left[\frac{1}{4 E_{2}^{s}}-\frac{1}{2 E_{2}^{s}\left(1+4 \rho^{2} \frac{E_{1}^{s}}{E_{2}^{s}}\right)}-\frac{4 \rho^{2}}{4 E_{2}^{s}\left(1-\rho^{2}\right)}-\frac{1}{4 E_{1}^{s}\left(1-\rho^{2}\right)}\right. \\
& \left.-\frac{4 \rho^{2} 2 E_{1}^{s}}{2 E_{2}^{s}+4 \rho^{2} 2 E_{1}^{s}} \frac{1}{2 E_{2}^{s}+4 \rho^{2} 2 E_{1}^{s}} \frac{1+4 \rho^{2} \frac{E_{1}^{s}}{E_{2}^{s}}}{2}+\frac{\frac{4 \rho^{2}}{\left(1-\rho^{2}\right) 2 E_{2}^{s}}}{2} \frac{1-\left(1+4 \rho^{2} \frac{E_{1}^{s}}{E_{2}^{s}}\right)^{-1 / 2}}{\frac{4 \rho^{2} \frac{E_{1}^{s}}{E_{2}^{s}}}{1+4 \rho^{2} \frac{E_{1}^{s}}{E_{2}^{s}}}}\right] \\
& =\frac{1}{8 E_{2}^{s}}+\frac{1}{8} \frac{1}{2 E_{1}^{s}\left(1-\rho^{2}\right)} \\
& +\frac{1}{4} \frac{1}{\left(1+4 \rho^{2} \frac{E_{1}^{s}}{E_{2}^{s}}\right)^{1 / 2}}\left[\frac{1}{4 E_{2}^{s}}-\frac{1}{2 E_{2}^{s}\left(1+4 \rho^{2} \frac{E_{1}^{s}}{E_{2}^{s}}\right)}-\frac{4 \rho^{2}}{4 E_{2}^{s}\left(1-\rho^{2}\right)}-\frac{1}{4 E_{1}^{s}\left(1-\rho^{2}\right)}\right. \\
& \left.-\frac{4 \rho^{2} 2 E_{1}^{s}}{2 E_{2}^{s}\left(1+4 \rho^{2} \frac{E_{1}^{s}}{E_{2}^{s}}\right)} \frac{1}{4 E_{2}^{s}}+\frac{\frac{4 \rho^{2}}{\left(1-\rho^{2}\right) 2 E_{2}^{s}}}{2} \frac{1-\left(1+4 \rho^{2} \frac{E_{1}^{s}}{E_{2}^{s}}\right)^{-1 / 2}}{\frac{4 \rho^{2} \frac{E_{s}^{s}}{E_{2}^{s}}}{1+4 \rho^{2} \frac{E_{1}^{s}}{E_{2}^{s}}}}+\frac{1}{4 E_{2}^{s}\left(1+4 \rho^{2} \frac{E_{1}^{s}}{E_{2}^{s}}\right)}\right] \\
& =\frac{1}{8 E_{2}^{s}}+\frac{1}{8} \frac{1}{2 E_{1}^{s}\left(1-\rho^{2}\right)} \\
& +\frac{1}{4} \frac{1}{\left(1+4 \rho^{2} \frac{E_{1}^{s}}{E_{2}^{s}}\right)^{1 / 2}}\left[\frac{\frac{1}{2} 4 \rho^{2} \frac{E_{1}^{s}}{E_{2}^{s}}}{2 E_{2}^{s}\left(1+4 \rho^{2} \frac{E_{1}^{s}}{E_{2}^{s}}\right)}-\frac{1+4 \rho^{2} \frac{E_{1}^{s}}{E_{2}^{s}}}{4 E_{1}^{s}\left(1-\rho^{2}\right)}-\frac{\frac{1}{2} 4 \rho^{2} \frac{E_{1}^{s}}{E_{2}^{s}}}{2 E_{2}^{s}\left(1+4 \rho^{2} \frac{E_{1}^{s}}{E_{2}^{s}}\right)}\right. \\
& \left.+\frac{4 \rho^{2}\left(1+4 \rho^{2} \frac{E_{1}^{s}}{E_{2}^{s}}\right)}{4 \rho^{2}\left(1-\rho^{2}\right) 4 E_{2}^{s} \frac{E_{1}^{s}}{E_{2}^{s}}}\left(1-\left(1+4 \rho^{2} \frac{E_{1}^{s}}{E_{2}^{s}}\right)^{-1 / 2}\right)\right] \\
& =\frac{1}{8 E_{2}^{s}}+\frac{1}{8} \frac{1}{2 E_{1}^{s}\left(1-\rho^{2}\right)} \\
& +\frac{1}{4} \frac{1}{\left(1+4 \rho^{2} \frac{E_{1}^{s}}{E_{2}^{s}}\right)^{1 / 2}}\left[-\frac{1+4 \rho^{2} \frac{E_{1}^{s}}{E_{2}^{s}}}{4 E_{1}^{s}\left(1-\rho^{2}\right)}+\frac{\left(1+4 \rho^{2} \frac{E_{1}^{s}}{E_{2}^{s}}\right)}{\left(1-\rho^{2}\right) 4 E_{1}^{s}}-\frac{\left(1+4 \rho^{2} \frac{E_{1}^{s}}{E_{2}^{s}}\right)^{1 / 2}}{\left(1-\rho^{2}\right) 4 E_{1}^{s}}\right] \\
& =\frac{1}{8 E_{2}^{s}}+\frac{1}{8} \frac{1}{2 E_{1}^{s}\left(1-\rho^{2}\right)}-\frac{1}{4\left(1-\rho^{2}\right) 4 E_{1}^{s}}=\frac{1}{8 E_{2}^{s}}
\end{aligned}
$$


since

$$
\begin{aligned}
1-\gamma & =1-\frac{4 \rho^{2} 2 E_{1}^{s}}{\left(2 E_{2}^{s}+4 \rho^{2} 2 E_{1}^{s}\right)}=\frac{2 E_{2}^{s}}{2 E_{2}^{s}+4 \rho^{2} 2 E_{1}^{s}}=\frac{1}{1+4 \rho^{2} \frac{E_{1}^{s}}{E_{2}^{s}}} \\
\delta & =\frac{1}{2 E_{2}^{s}+4 \rho^{2} 2 E_{1}^{s}} \quad \mu=\frac{4 \rho^{2}}{\left(1-\rho^{2}\right) 2 E_{2}^{s}}
\end{aligned}
$$




\section{References}

[1] S. Chatterjee, W. Fernando, and M. Wasantha, "Adaptive modulation based MC-CDMA systems for 4G wireless consumer applications," IEEE Trans. on Consumer Electronics, vol. 49, pp. 995-1003, Nov. 2003.

[2] S. Verdu, Multiuser detection. Cambridge University press, 1998.

[3] T. Ottosson and A. Svensson, "Multi-rate schemes in DS/CDMA systems," $45^{\text {th }}$ IEEE Vehicular Technology Conference (VTC95'), 1995.

[4] M. Juntti, "System concept comparisons for multirate CDMA with multiuser detection," 48 $8^{\text {th }}$ IEEE Vehicular Technology Conference (VTC98'), pp. 36-40, May 1998.

[5] D.-S. Yoo and W. Stark, "Interference cancellation for multirate multiuser systems," 53 ${ }^{\text {th }}$ IEEE Vehicular Technology Conference (VTCS01'), vol. 3, pp. 1584-1588, May 2001.

[6] M. Juntti and B. Aazhang, "Finite memory-length linear multiuser detection for asynchronous CDMA communications," IEEE Trans. on Comm., vol. 45, pp. 611-622, May 1997.

[7] U. Mitra, "Comparison of maximum-likelihood-based detection for two multirate access schemes for CDMA signals," IEEE Trans. on Comm., vol. 47, pp. 64-77, Jan. 1999.

[8] M. Saquib, R. Yates, and N. Mandayam, "Decorrelating detectors for a dual rate synchronous DS/CDMA system," Wireless Personal Communications, vol. 9, pp. 197-216, May 1999.

[9] J. Proakis, Digital communications, 4th Ed. New York: McGraw-Hill Inc., 2000.

[10] P. Dent, B. Gudmundson, and M. Ewerbring, "CDMA-IC: a novel code division multiple access scheme based on interference cancellation," $3^{\text {rd }}$ IEEE International Symposium on Personal, Indoor and Mobile Radio Communications (PIMRC'92), pp. 98-102, 1992. 
[11] M. Chen and Y. Li, "Multiuser detection in multirate DS/CDMA communications," Proc. IEEE Milcom Conf., vol. 2, pp. 583-587, Oct. 2000.

[12] T. Minn and K.-Y. Siu, "Variable chip rate CDMA," 51 $1^{\text {th }}$ IEEE Vehicular Technology Conference (VTCS00'), vol. 3, pp. 2267-2271, May 2000.

[13] S. Moshavi, "Multi-user detection for DS-CDMA communications," IEEE Communications Magazine, vol. 34, pp. 124-136, Oct. 1996.

[14] D. Koulakiotis and A. Aghvami, "Data detection techniques for DS/CDMA mobile systems: a review," IEEE Personal Communications, vol. 7, pp. 24-34, June 2000.

[15] S. Verdu, "Minimum probability of error for asynchronous Gaussian multiple access channels," IEEE Trans. on Inform. Theory, vol. 32, pp. 85-96, Jan. 1986.

[16] A. Voorman, D. Koulakiotis, A. Aghvami, and R. Prasad, "Performance evaluation of the decorrelating detector for DS-CDMA systems over multipath Rayleigh fading channels with AWGN," $9^{\text {th }}$ IEEE International Symposium on Personal, Indoor and Mobile Radio Communications (PIMRC'98), vol. 1, pp. 228-232, 1998.

[17] A. Haghighat and M. Soleymani, "An improved decorrelator-based multiuser receiver," IEEE Military Communications Conference (MILCOM 2001), vol. 2, pp. 1105-1108, 2001.

[18] M. Juntti, B. Aazhang, and J. Lilleberg, "Iterative implementation of linear multiuser detection for dynamic asynchronous CDMA systems," IEEE Trans. on Comm., vol. 46, pp. 503-508, Apr. 1998.

[19] R. He, I. Reed, and T. Truong, "A recursive linear detection algorithm for asynchronous cdma communication system," IEEE Trans. on Inform. Theory, vol. 48 , pp. 1835-1848, July 2002.

[20] L. Wei and L. Rasmussen, "A near ideal noise whitening filter for an asynchronous time-varying CDMA system," IEEE Trans. on Comm., vol. 44, pp. 1355-1361, Oct. 1996.

[21] A. Duel-Hallen, "A family of multiuser decision-feedback detectors for asynchronous code-division multiple-access channels," IEEE Trans. on Comm., vol. 43, pp. 421-434, Feb/Mar/Apr 1995.

[22] S. Maeng and B. Lee, "Extended-window decorrelating detector for asynchronous CDMA channels and its performance analysis," IEEE Trans. on Comm., vol. 49, pp. 35-40, Jan. 2001. 
[23] J. Shen and Z. Ding, "Edge decision assisted decorrelators for asynchronous CDMA channels," IEEE Trans. on Comm., vol. 47, pp. 438-445, Mar. 1999.

[24] S.-K. Lee and S. Kim, "A one-shot LDD with pre-SDC/post-MRC for random CDMA over asynchronous Rayleigh channels," IEEE Journal on Selected Areas in Communications, vol. 20, pp. 257-263, Feb. 2002.

[25] W. Ye and P. Varshney, "A two-stage decorrelating detector for DS/CDMA systems," IEEE Trans. on Vehicular Technology, vol. 50, pp. 465-479, Mar. 2001.

[26] L. Rugini, P. Banelli, and S. Cacopardi, "Theoretical analysis and performance of the decorrelating detector for DS-CDMA signals in nonlinear channels," IEEE Trans. Wireless Communications, vol. 3, pp. 367-372, March 2004.

[27] Z. Xie, R. Short, and C. Rushforth, "A family of suboptimum detectors for coherent multiuser communications," IEEE Journal on Selected Areas in Communications, vol. 8, pp. 683-690, May 1990.

[28] U. Madhow and M. Honig, "MMSE interference suppression for direct-sequence spread-spectrum CDMA," IEEE Trans. on Comm., vol. 42, pp. 3178-3188, Dec. 1994.

[29] H. Poor and S. Verdu, "Probability of error in MMSE multiuser detection," IEEE Trans. on Inform. Theory, vol. 43, pp. 858-871, May 1997.

[30] W. Hamouda and P. McLane, "A fast adaptive algorithm for MMSE receivers in DS-CDMA systems," IEEE Signal Processing Letters, vol. 11, pp. 86-89, Feb 2004.

[31] M. Honig, U. Madhow, and S. Verdu, "Blind adaptive multiuser detection," IEEE Trans. on Inform. Theory, vol. 41, pp. 944-960, July 1995.

[32] W. Chen and U. Mitra, "An improved blind adaptive MMSE receiver for fast fading DS-CDMA channels," Proc. IEEE Globecom Conf., vol. 2, pp. 758-762, Nov. 2001.

[33] P. Patel and J. Holtzman, "Analysis of a simple successive interference cancellation scheme in a DS/CDMA system," IEEE Journal on Selected Areas in Communications, vol. 12, pp. 796-807, June 1994.

[34] K. Puttegowda, G. Verma, S. Bali, and R. Buehrer, "On the effect of cancellation order in successive interference cancellation for CDMA systems," $58^{\text {th }}$ IEEE Vehicular Technology Conference (VTCF03'), vol. 2, pp. 1035-1039, Oct. 2003. 
[35] M. Ammar, T. Chonavel, and S. Saoudi, "Multi-stage SIC structure for uplink UMTS multiuser receiver over multipath Rayleigh channels," $53^{\text {th }}$ IEEE Vehicular Technology Conference (VTCS01'), vol. 4, pp. 2514-2518, May 2001.

[36] H. Elders-Boll, H. Schotten, and A. Busboom, "Implementation of linear multiuser detectors for asynchronous CDMA systems by linear multi-stage interference cancellation," Proceedings of the 1998 IEEE International Conference on Acoustics, Speech, and Signal Processing, 1998, vol. 6, pp. 3225-3228, May 1998.

[37] A. Hui and K. Letaief, "Successive interference cancellation for multiuser asynchronous DS/CDMA detectors in multipath fading links," IEEE Trans. on Comm., vol. 46, pp. 384-391, Mar. 1998.

[38] A. Lampe and J. Huber, "On improved multiuser detection with iterated soft decision interference cancellation," Communication Theory Mini-Conference, vol. 6-10, pp. 172-176, June 1999.

[39] W. Zha and S. Blostein, "Soft-decision successive interference cancellation CDMA receiver with amplitude averaging and robust to timing errors," IEEE Global Telecommunications Conference, 2001, vol. 2, pp. 743-747, Nov. 2001.

[40] M. Kobayashi, J. Boutros, and G. Caire, "Successive interference cancellation with SISO decoding and EM channel estimation," IEEE Journal on Selected Areas in Communications, vol. 19, pp. 1450-1460, Aug. 2001.

[41] J. Andrews and T. Meng, "Multiple access interference cancellation in fading multipath channels: progress and limitations," $53^{\text {th }}$ IEEE Vehicular Technology Conference (VTCS01'), vol. 1, pp. 614-618, May 2001.

[42] M. Varanasi and B. Aazhang, "Multistage detection in asynchronous codedivision multiple-access communications," IEEE Trans. on Comm., vol. 38, pp. 509-519, Apr. 1990.

[43] Y. Yoon, R. Kohno, and H. Imai, "A spread-spectrum multiaccess system with cochannel interference cancellation for multipath fading channels," IEEE Journal on Selected Areas in Communications, vol. 11, pp. 1067-1075, Sept. 1993.

[44] M. Juntti and M. Latva-aho, "Multiuser receivers for CDMA systems in Rayleigh fading channels," IEEE Trans. on Vehicular Technology, vol. 49, pp. 885-899, May 2000.

[45] K. Ko, M. Joo, H. Lee, and D. Hong, "Performance analysis for multistage interference cancellers in asynchronous DS-CDMA systems," IEEE Communications Letters, vol. 6, pp. 544-546, Dec 2002. 
[46] D. Divsalar, M. Simon, and D. Raphaeli, "Improved parallel interference cancellation for CDMA," IEEE Trans. on Comm., vol. 46, pp. 258-268, Feb. 1998.

[47] M. Ghotbi and M. Soleymani, "Multiuser detection of DS-CDMA signals using partial parallel interference cancellation in satellite communications," IEEE Journal on Selected Areas in Communications, vol. 22, pp. 584-593, April 2004.

[48] T.-H. Liu, "Decision-feedback multiuser detection with orthogonal feedforward transformation," IEEE Trans. on Vehicular Technology, vol. 53, pp. 1370-1378, Sept 2004.

[49] M. Wang, J. Lu, Z. Zhou, and J. Wang, "A new interference cancellation receiver in fading channels," The 8th International Conference on Communication Systems, vol. 1, pp. 573-577, Nov. 2002.

[50] C.-H. Tsai, W.-H. Fang, and J.-T. Chen, "Hybrid MMSE and SIC for multiuser detection," 53 ${ }^{\text {th }}$ IEEE Vehicular Technology Conference (VTCS01'), vol. 3, pp. 1779-1783, May 2001.

[51] J. Kim and S. Kim, "Partial successive interference cancellation in hybrid DS/FH spread-spectrum multiple-access systems," IEEE Trans. on Comm., vol. 49, pp. 1710-1714, Oct. 2001.

[52] J. Kim, J. Jeong, S. Yeom, B. Choi, and Y. Park, "Performance analysis of the hybrid interference canceller for multiple access interference cancellation," TENCON 99, Proceedings of the IEEE Region 10 Conference, vol. 2, pp. 12361239, Sept 1999.

[53] L. Nithyanandan, R. Saravanaprabu, and P. Dananjayan, "Hybrid interference cancellation receiver for MC-CDMA system," 2005 IEEE International Conference on Personal Wireless Communications, 2005. ICPWC 2005., pp. 465-469, Jan. 2005.

[54] S. Sun, L. Rasmussen, H. Sugimoto, and T. Lim, "A hybrid interference canceller in CDMA," Proceedings of IEEE 5th International Symposium onSpread Spectrum Techniques and Applications, vol. 1, pp. 150-154, Sept 1998.

[55] R. Buehrer, N. Correal-Mendoza, and B. Woerner, "A simulation comparison of multiuser receivers for cellular CDMA," IEEE Trans. on Vehicular Technology, vol. 49, pp. 1065-1085, July 2000 .

[56] M. Fan, C. Hoffmann, and K.-Y. Siu, "Error-Rate analysis for multirate DSCDMA transmission schemes," IEEE Trans. on Comm., vol. 51, pp. 1897-1909, Nov. 2003. 
[57] M. Fan, T. Minn, and K.-Y. Siu, "Exact bit-error rate analysis of multirate DS-CDMA schemes," 2002 IEEE Seventh International Symposium on Spread Spectrum Techniques and Applications, vol. 2, pp. 415-419, Sept 2002.

[58] P.-W. Fu and K.-C. Chen, "Multiuser detection for multi-rate CDMA in multipath fading channels," $52^{\text {th }}$ IEEE Vehicular Technology Conference (VTCF00'), 2000 .

[59] Z. Guo and K. Letaief, "Performance of multiuser detection in multirate DSCDMA systems," IEEE Trans. on Comm., vol. 51, pp. 1979-1983, Dec. 2003.

[60] L. Lupas and S. Verdu, "Linear multiuser detectors for synchronous CodeDivision Multiple-Access systems," IEEE Trans. on Inform. Theory, vol. 35, pp. 123-136, Jan. 1989.

[61] P. Zong, Y. Bar-Ness, and J. Chan, "Performance analysis of a dual-rate synchronous MC-CDMA system," IEEE Global Telecommunications Conference, 2000, vol. 3, pp. 1380-1384, Nov. 2000.

[62] J. Chen and U. Mitra, "Analysis of decorrelator-based receivers for multirate DS/CDMA communications," IEEE Trans. on Vehicular Technology, vol. 48, pp. 1966-1963, Nov. 1999.

[63] M. Saquib, R. Yates, and A. Ganti, "An asynchronous multirate decorrelator," IEEE Trans. on Comm., vol. 48, pp. 739-742, May 2000.

[64] I. Barbancho, A. Barbancho, L. Tardbn, and J. Emtrambasaguas, "Multirate decorrelating detector for UMTS," IEEE MELECON 2004, pp. 469-472, May 2004.

[65] M. Madkour and S. Gupta, "Performance analysis of a wireless multirate directsequence CDMA using fast Walsh transform and decorrelating detection," IEEE Trans. on Comm., vol. 48, pp. 1405-1411, Aug. 2000.

[66] M. Saquib, R. Yates, and A. Ganti, "Power control for an asynchronous multirate decorrelator," IEEE Trans. on Comm., vol. 48, pp. 804-812, May 2000.

[67] H. Lee and K.-C. Chen, "Performance analysis and improvement of decorrelating detection for multi-rate DS/CDMA," IEEE Communications Letters, vol. 9, pp. 103-105, Feb 2005.

[68] H. Park, J. Kim, and J. Kim, "Multirate decorrelating detector for a multicarrier DS/CDMA mobile multimedia system," Intelligent Signal Processing and Communication Systems, 2004. ISPACS 2004. Proceedings of 2004 International Symposium on, pp. 685-689, Nov 2004. 
[69] A. Sabharwal, U. Mitra, and R. Moses, "MMSE receivers for multirate DSCDMA systems," IEEE Trans. on Comm., vol. 49, pp. 2184-2197, Dec. 2001.

[70] M. El-Khamy, S. Elnoubi, and O. Alim, "Centralized adaptive MMSE detectors for multirate DS-CDMA in multipath fading channels," Proceedings of the Nineteenth National Radio Science Conference (NRSC 2002), pp. 381-388, Mar. 2002.

[71] J. Choi and S. Kim, "Adaptive MMSE receiver for multirate CDMA systems," IEEE Trans. on Signal Processing, vol. 50, pp. 3098-3106, Dec. 2002.

[72] Q. Du, S. Zhu, and P. Ren, "An improved adaptive MMSE algorithm for multirate multiuser detection in WCDMA systems," IEEE 6th CAS Symp. on Emerging Technologies: Mobile and Wireless COMM, pp. 317-320, May 2004.

[73] J. Fonollosa and X. Mestre, "Exact bit-error rate analysis of multirate DSCDMA schemes," 2002 IEEE Seventh International Symposium on Spread Spectrum Techniques and Applications, vol. 2, pp. 415-419, Sept 2002.

[74] M.-H. Chung, K.-C. Chen, and M. You, "MMSE multiuser detection for multirate wideband CDMA communications," The 11th IEEE International Symposium on Personal, Indoor and Mobile Radio Communications, vol. 1, pp. 534538 , Sept 2000.

[75] J. Ma and J. Tugnait, "Blind detection of multirate asynchronous CDMA signals in multipath channels," IEEE Trans. on Signal Processing, vol. 50, pp. 22582272, Sep 2002.

[76] L. Tong, A. van der Veen, P. Dewilde, and Y. Sung, "Blind decorrelating RAKE receivers for long-code WCDMA," IEEE Journal on Selected Areas in Communications, vol. 51, pp. 303-309, June 2003.

[77] A. Johansson, T. Ottosson, and A. Svensson, "On multirate DS/CDMA with interference cancellation for wireless multimedia applications," IEEE International Conference on Personal Wireless communications, pp. 102-107, Feb. 1996.

[78] T. Abrao and P. Jeszensky, "Successive parallel interference canceller for asynchronous multirate ds-cdma systems," $13^{\text {th }}$ IEEE International Symposium on Personal, Indoor and Mobile Radio Communications (PIMRC'02), vol. 5, pp. 2003-2005, Sept. 2002.

[79] M. Madkour, S. Gupta, and Y. Wang, "Successive interference cancellation algorithms for downlink W-CDMA communications," IEEE Trans. Wireless Communications, vol. 1, pp. 169-177, Jan. 2002. 
[80] M. Juntti, "Multiuser detector performance comparisons in multirate CDMA systems," $48^{\text {th }}$ IEEE Vehicular Technology Conference (VTC98'), pp. 31-35, May 1998.

[81] F. Berggren and S. Slimane, "Successive interference cancellation in multi-rate DS-CDMA systems," 14th IEEE Proceedings on Personal, Indoor and Mobile Radio Communications, vol. 2, pp. 1752-1756, Sept 2003.

[82] A. Boariu and R. Ziemer, "Multiuser detection in multipath environments for variable spreading-factor CDMA systems," IEEE Trans. on Comm., vol. 49, pp. 1520-1524, Sept. 2001.

[83] H. Yan and S. Roy, "Parallel interference cancellation for uplink multirate overlay CDMA channels," IEEE Trans. on Comm., vol. 53, pp. 152-161, Jan 2005.

[84] M. Alam, T. Ojanpera, and R. Prasad, "Near-far resistance of parallel interference cancellation detector in a multirate DS-CDMA systems," $50^{\text {th }}$ IEEE Vehicular Technology Conference (VTCF99'), vol. 3, pp. 1830-1834, Sept 1999.

[85] S. Han and J. Lee, "Group-wise successive interference cancellation receiver with adaptive MMSE detection for dual-rate DS-CDMA system," IEEE Global Telecommunications Conference, 2002, vol. 1, pp. 514-518, Nov. 2002.

[86] N. Kim and M. Howlader, "Analysis of a new hybrid interference cancellation (HIC) system," Wireless Communications and Networking Conference, 2004. WCNC. 2004 IEEE, vol. 3, pp. 1828-1832, March 2004.

[87] A. Pais, K. Sowerby, and M. Neve, "Implications of power control and successive interference cancellation on indoor ds-cdma system deployment and performance," IEEE Communications Letters, vol. 9, pp. 204-206, Mar. 2006.

[88] Y. Huang and T. Ng, "DS-CDMA with power control error using weighted despreading sequences over a multipath rayleigh fading channel," IEEE Trans. on Vehicular Technology, vol. 48, pp. 1067-1079, July 1999.

[89] R. Cameron and B. Woerner, "Performance analysis of CDMA with imperfect power control," IEEE Trans. on Comm., vol. 44, pp. 777-781, July 1996.

[90] A. A. Paul, M. Akar, M. Safonov, and U. Mitra, "Adaptive power control for wireless networks using multiple controllers and switching," IEEE Trans. Wireless Communications, vol. 16, pp. 1212-1218, Sept. 2005.

[91] J. Panicker and S. Kumar, "Effect of system imperfections on BER performance of CDMA correlator receiver," Proc. IEEE Int. Conf. Communications (ICC), vol. 2, pp. 894-899, June 1995. 
[92] D. Warrier and U. Madhow, "On the capacity of cellular CDMA with successive decoding and controlled power disparities," $48^{\text {th }}$ IEEE Vehicular Technology Conference (VTC98'), vol. 3, pp. 1873-1877, May 1998.

[93] J. Andrews and T. Meng, "Optimum power control for successive interference cancellation with imperfect channel estimation," IEEE Transactions on Wireless Communications., vol. 2, pp. 375-383, March 2003.

[94] J. Kim and S. Kim, "Combined power control and successive interference cancellation in DS/CDMA communications," The 5th International Symposium on Wireless Personal Multimedia Communications, 2002, vol. 3, pp. 931-935, Oct 2002.

[95] S. Gray and D. Brady, "Multiuser detection in mismatched multiple-access channels," IEEE Trans. on Comm., vol. 43, pp. 3080-3089, Dec 1995.

[96] R. Buehrer and B. Woerner, "The asymptotic multiuser efficiency of M-stage interference cancellation receivers," $8^{\text {th }}$ IEEE International Symposium on Personal, Indoor and Mobile Radio Communications (PIMRC'97), vol. 2, pp. 570574, Sept 1997.

[97] X. Zhang, "Asymptotic multiuser efficiency for decision-feedback multiuser detection," 1996 IEEE International Conference on Communication (ICC), vol. 43, pp. 359-363, June 1996.

[98] X. Zhang and D. Brady, "Asymptotic multiuser efficiencies for decision-directed multiuser detectors," IEEE Trans. on Inform. Theory, vol. 44, pp. 502-515, Mar 1998.

[99] L. Chu and U. Mitra, "Performance analysis of an improved MMSE multiuser receiver for mismatched delay channels," IEEE Trans. on Comm., vol. 46, pp. 1369-1380, Oct 1998.

[100] K. Lai and J. Shynk, "Performance evaluation of a generalized linear SIC for DS-CDMA signals," IEEE Trans. Signal Processing, vol. 51, pp. 1604-1614, June 2003.

[101] B. Yang and F. Danilo-Lemoine, "Performance of a decorrelator based successive interference cancellation multiuser receiver for asynchronous multirate DSCDMA systems," Military Communication Conference (MILCOM), Oct 2005.

[102] S. Bensley and B. Aazhag, "Subspace-based channel estimation for code-division multiple access communication systems," IEEE Trans. on Comm., vol. 44, pp. 1009-1020, Aug. 1996. 
[103] Z. Xie, C. Rushforth, R. Short, and T. Moon, "Joint signal and parameter estimation in multiuser communications," IEEE Trans. on Comm., vol. 41, pp. 1208-1276, Aug. 1993.

[104] A. P. Dempster, N. M. Laird, and D. B. Rubin, "Maximum-likelihood from incomplete data via the EM algorithm," J.Roy.Statist. Soc., vol. 39, pp. 1-17, 1977.

[105] G. Ye and G. Bi, "An efficient multipath channel estimator for DS-CDMA systems," Proc. IEEE Int. Conf. Communications (ICC), vol. 3, pp. 1613-1616, June 1999.

[106] E. Ertin, U. Mitra, and S. Siwamogsatham, "Maximum-Likelihood-based multipath channel estimation for code-division multiple access systems," IEEE Trans. on Comm., vol. 49, pp. 290-302, Feb. 2001.

[107] A. Rizaner, H. Amca, and K. H. A. Ulusoy, "Channel estimation using short training sequences," 52 $2^{\text {th }}$ IEEE Vehicular Technology Conference (VTCF00'), vol. 6, pp. 2630-2633, Sept 2000.

[108] E. Papproth and G. Kaleh, "Near-far resistant channel estimation for the DSCDMA uplink," $6^{\text {th }}$ IEEE International Symposium on Personal, Indoor and Mobile Radio Communications (PIMRC'95), vol. 2, pp. 758-762, Sept 1995.

[109] S. Bhashyam and B. Aazhang, "Multiuser channel estimation and tracking for long-code CDMA systems," IEEE Trans. on Comm., vol. 50, pp. 1081-1090, Jul 2002.

[110] G. Fock, J. Baltersee, P. Schulz-Rittich, and H. Meyr, "Channel tracking for RAKE receivers in closely spaced multipath environments," IEEE Journal on Selected Areas in Communications, vol. 19, pp. 2420-2431, Dec. 2001.

[111] S. Bhashyam, A. Sabharwal, and U. Mitra, "Channel estimation for multirate DS-CDMA systems," Conference Record of the Thirty-Fourth Asilomar Conference on Signals, Systems and Computers, 2000, vol. 2, pp. 960-964, Oct 2000.

[112] P. Liu and Z. Xu, "Diversity-Assisted channel estimation and multiuser detection for downlink CDMA with long spreading codes," IEEE Trans. Signal Processing, vol. 52, pp. 190-201, Jan. 2004.

[113] S. Roy and H. Yan, "Blind channel estimation in multi-rate CDMA systems," IEEE Trans. on Comm., vol. 50, pp. 995-1004, June 2002.

[114] L. Huang, F. Zheng, and M. Faulkner, "Blind adaptive channel estimation for dual-rate DS/CDMA signals," IEEE Communications Letters, vol. 6, pp. 129131, Apr 2002. 
[115] A. Lampe and C. Windpassinger, "Multiuser detection with iterated channel estimation," Second International Workshop on Multi-Carrier Spread-Spectrum and Related Topics, July 1999.

[116] Q. Li, C. Georghiades, and X. Wang, "Blind multiuser detection in uplink CDMA with multipath fading: a sequential EM approach," IEEE Trans. on Comm., vol. 52, pp. 71-81, Jan. 2004.

[117] S.-H. Wu, U. Mitra, and C.-C. Kuo, "Joint channel estimation and multiuser detection for multipath fading channels in DS-CDMA," Proc. IEEE Int. Conf. Communications (ICC), vol. 4, pp. 2411-2415, May 2003.

[118] C. Sengupta, S. Das, J. Cavallaro, and B. Aazhang, "Joint multiuser channel estimation and detection for CDMA systems," IEEE Information Theory Workshop on Detection, Estimation, Classification and Imaging, Feb 1999.

[119] P. Xiao, E. Strom, and R. Carrasco, "Estimation of time-varying multipath Rayleigh fading channels in asynchronous DS-CDMA systems," $15^{\text {th }}$ IEEE International Symposium on Personal, Indoor and Mobile Radio Communications (PIMRC'04), vol. 4, pp. 2570-2575, Sept 2004.

[120] M.-A. Baissas and A. Sayeed, "Pilot-based estimation of time-varying multipath channels for coherent CDMA receivers," IEEE Trans. Signal Processing, vol. 50, pp. 2037-2049, Aug 2002.

[121] G. Yue, X. Zhou, and X. Wang, "Performance comparisons of channel estimation techniques in multipath fading CDMA," IEEE Trans. Wireless Communications, vol. 3, pp. 716-724, May 2004.

[122] C. D'Amours, M. Moher, and A. Yongacoglu, "Comparison of pilot symbolassisted and differentially detected BPSK for DS-CDMA systems employing RAKE receivers in Rayleigh fading channels," IEEE Trans. on Vehicular Technology, vol. 47, pp. 1258-1267, Nov. 1998.

[123] A. Weiss and B. Friedlander, "Channel estimation for DS-CDMA downlink with aperiodic spreading codes," IEEE Trans. on Comm., vol. 47, pp. 1561-1569, Oct 1999.

[124] J. Wu, C. Xiao, and K. Letaief, "Multiuser channel estimation for CDMA systems over frequency-selective fading channels," IEEE Trans. Wireless Communications, vol. 4, pp. 1724-1736, July 2005.

[125] E. Aktas and U. Mitra, "Single-user sparse channel acquisition in multiuser DS-CDMA systems," IEEE Trans. on Comm., vol. 51, pp. 682-693, Apr 2003. 
[126] M. Stojanovic and Z. Zvonar, "Performance of multiuser detection with adaptive channel estimation," IEEE Trans. on Comm., vol. 47, pp. 1129-1132, Aug 1999.

[127] T. Kawahara and T. Matsumoto, "Joint decorrelating multiuser detection and channel estimation in asynchronous CDMA mobile communications channels," IEEE Trans. on Vehicular Technology, vol. 44, pp. 506-515, Aug 1995.

[128] Y. Ma, R. Schober, S. Pasupathy, and T. Lim, "Efficient BER evaluation of linear multiuser detectors with imperfect channel estimation for CDMA fading channels," IEEE Trans. on Comm., vol. 54, pp. 242-250, Feb. 2006.

[129] E. Dinan and B. Jabbari, "Spreading codes for direct sequence CDMA and wideband CDMA cellular networks," IEEE Communications Magazine, vol. 36, pp. 48-54, Sept. 1998.

[130] T. Rappaport, Wireless communications: principles and practice, 2nd edition. Prentice Hall, 2002.

[131] B. Yang and F. Danilo-Lemoine, "A decorrelator based successive interference cancellation multiuser multirate receiver," Canadian Conference on Electrical and Computer Engineering, pp. 98-103, May 2005.

[132] B. Yang and F. Danilo-Lemoine, "Comparison of multiuser detection techniques for asynchronous multirate DS-CDMA systems," The 16th Annual IEEE International Symposium on Personal Indoor and Mobile Radio Communications, Sept 2005.

[133] B. Yang and F. Danilo-Lemoine, "Asymptotic multiuser efficiency of a decorrelator based successive interference cancellation DS-CDMA multiuser receiver," Military Communication Conference (MILCOM), Oct 2006.

[134] M. Abramowitz and I. Stegun, Handbook of Mathematical Functions With Formulas, Graphs, and Mathematical Tables, Ninth printing. UNITED STATES DEPARTMENT OF COMMERCE, NATIONAL BUREAU OF STANDARDS, 1970.

[135] I. Gradshteyn and I. Ryzhik, Table of integrals, series and products 6th edition, prepared by A. Jeffrey. New York: Academic Press, 2000. 